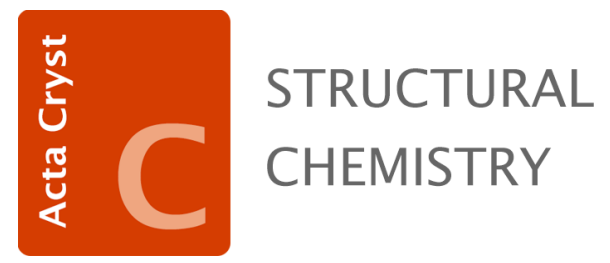

Volume 75 (2019)

Supporting information for article:

Asymmetric diosmium sawhorse complexes

Kylie M. Wilson, John W. Swartout, Henry A. Touchton, Erica N. Lambert, James E. Johnstone, Ashley K. Archambeau, David M. Marolf, Emily R. Mikeska, Vincent M. Lynch, Vladimir N. Nesterov, Eric W. Reinheimer, Gregory L. Powell and Cynthia B. Powell 


\section{Computing details}

Data collection: CrysAlis PRO 1.171.39.33b (Rigaku OD, 2017) for (1) $\cdot \mathrm{CH}_{2} \mathrm{Cl}_{2}$; CrystalClear (Rigaku Inc., 2008) for (3), (7) $\cdot \mathrm{CH}_{2} \mathrm{Cl}_{2}$; CrysAlis PRO 1.171.39.35c (Rigaku OD, 2017) for (4) $\cdot 1.5 \mathrm{C}_{8} \mathrm{H}_{10}$; CrysAlis PRO 1.171.38.43f (Rigaku OD, 2015) for (5), (6). Cell refinement: CrysAlis PRO 1.171.39.33b (Rigaku OD, 2017) for (1) $\cdot \mathrm{CH}_{2} \mathrm{Cl}_{2}$; SAINT v7.68A (Bruker, 2009) for (2), (8); CrystalClear (Rigaku Inc., 2008) for (3), (7) $\cdot \mathrm{CH}_{2} \mathrm{Cl}_{2}$; CrysAlis PRO 1.171.39.35c (Rigaku OD, 2017) for (4) $1.5 \mathrm{C}_{8} \mathrm{H}_{10}$; CrysAlis PRO 1.171.38.43f (Rigaku OD, 2015) for (5), (6). Data reduction: CrysAlis PRO 1.171.39.33b (Rigaku OD, 2017) for (1) $\cdot \mathrm{CH}_{2} \mathrm{Cl}_{2} ;$ SAINT v7.68A (Bruker, 2009) for (2), (8); CrystalClear (Rigaku Inc., 2008) for (3), (7) $\cdot \mathrm{CH}_{2} \mathrm{Cl}_{2}$; CrysAlis PRO 1.171.39.35c (Rigaku OD, 2017) for $(4) \cdot 1.5 \mathrm{C}_{8} \mathrm{H}_{10}$; CrysAlis PRO 1.171.38.43f (Rigaku OD, 2015) for (5), (6). Program(s) used to solve structure: ShelXT (Sheldrick, 2015) for (1) $\cdot \mathrm{CH}_{2} \mathrm{Cl}_{2}$, (4) $1.5 \mathrm{C}_{8} \mathrm{H}_{10}$; SIR97 - Altomare A., Burla M.C., Camalli M., Cascarano G.L., Giacovazzo C., Guagliardi A., Moliterni A.G.G., Polidori G.,Spagna R. for (3), (7) $\cdot \mathrm{CH}_{2} \mathrm{Cl}_{2}$; Program(s) used to refine structure: $\mathrm{XL}$ (Sheldrick, 2008) for (1) $\cdot \mathrm{CH}_{2} \mathrm{Cl}_{2},(4) \cdot 1.5 \mathrm{C}_{8} \mathrm{H}_{10},(5),(6)$; SHELXL (Sheldrick, 2015b) for (2), (8); SHELXL2013 (Sheldrick, 2013) for (3), (7) $\cdot \mathrm{CH}_{2} \mathrm{Cl}_{2}$. Molecular graphics: OLEX2 (Dolomanov et al., 2009) for (1) $\cdot \mathrm{CH}_{2} \mathrm{Cl}_{2},(2),(4) \cdot 1.5 \mathrm{C}_{8} \mathrm{H}_{10},(5),(6),(8) ; X P$ SHELXTL/PC, Siemens Analytical for (3), (7) $\cdot \mathrm{CH}_{2} \mathrm{Cl}_{2}$. Software used to prepare material for publication: OLEX2 (Dolomanov et al., 2009) for (1) $\cdot \mathrm{CH}_{2} \mathrm{Cl}_{2},(2),(4) \cdot 1.5 \mathrm{C}_{8} \mathrm{H}_{10},(5),(6),(8) ; X P S H E L X T L / P C$, Siemens Analytical for (3), (7) $\cdot \mathrm{CH}_{2} \mathrm{Cl}_{2}$.

\section{(1) $\cdot \mathrm{CH}_{2} \mathrm{Cl}_{2}$}

Crystal data

\begin{tabular}{|l|l|}
\hline $\mathrm{C}_{51} \mathrm{H}_{50} \mathrm{O}_{8} \mathrm{Os}_{2} \mathrm{P}_{2} \cdot \mathrm{CH}_{2} \mathrm{Cl}_{2}$ & $F(000)=2576$ \\
\hline$M_{r}=1318.17$ & $D_{\mathrm{x}}=1.725 \mathrm{Mg} \mathrm{m}^{-3}$ \\
\hline Monoclinic, $P 2_{1} / c$ & Cu Ka radiation, $\lambda=1.54184 \AA$ \\
\hline$a=18.5007(4) \AA$ & Cell parameters from 12326 reflections \\
\hline$b=18.5396(4) \AA$ & $\theta=4.5-73.3^{\circ}$ \\
\hline$c=14.8090(3) \AA$ & $\mu=11.30 \mathrm{~mm}^{-1}$ \\
\hline$\beta=91.9976(17)^{\circ}$ & $T=100 \mathrm{~K}$ \\
\hline
\end{tabular}




\begin{tabular}{|l|l|}
\hline$V=5076.35(18) \AA^{3}$ & Plank, colorless \\
\hline$Z=4$ & $0.22 \times 0.06 \times 0.03 \mathrm{~mm}$ \\
\hline
\end{tabular}

\section{Data collection}

\begin{tabular}{|l|l|}
\hline $\begin{array}{l}\text { Rigaku SuperNova AtlasS2 CCD } \\
\text { diffractometer }\end{array}$ & 8475 reflections with $I>2 \mathrm{~s}(I)$ \\
\hline Detector resolution: 5.2387 pixels $\mathrm{mm}^{-1}$ & $R_{\text {int }}=0.024$ \\
\hline $\omega$ scans & $\theta_{\max }=68.3^{\circ}, \theta_{\min }=5.6^{\circ}$ \\
\hline $\begin{array}{l}\text { Absorption correction: gaussian CrysAlis } \\
P R O \text { (Rigaku OD, 2017) }\end{array}$ & $h=-21 \rightarrow 22$ \\
\hline$T_{\min }=0.428, T_{\max }=1.000$ & $k=-19 \rightarrow 22$ \\
\hline 20129 measured reflections & $I=-17 \rightarrow 14$ \\
\hline 9265 independent reflections & \\
\hline
\end{tabular}

\section{Refinement}

\begin{tabular}{|l|l|}
\hline Refinement on $F^{2}$ & 112 restraints \\
\hline Least-squares matrix: full & $\begin{array}{l}\text { Hydrogen site location: inferred from } \\
\text { neighbouring sites }\end{array}$ \\
\hline$R\left[F^{2}>2 \mathrm{~s}\left(F^{2}\right)\right]=0.023$ & $\mathrm{H}$-atom parameters constrained \\
\hline$W R\left(F^{2}\right)=0.055$ & $\begin{array}{l}W=1 /\left[\sigma^{2}\left(F_{0}^{2}\right)+(0.0214 P)^{2}+5.3085 P\right] \\
\text { where } P=\left(F_{0}^{2}+2 F_{c}^{2}\right) / 3\end{array}$ \\
\hline$S=1.00$ & $(\Delta / \sigma)_{\max }=0.002$ \\
\hline 9265 reflections & $\Delta\rangle_{\max }=1.16 \mathrm{e} \AA^{-3}$ \\
\hline 632 parameters & $\Delta\rangle_{\min }=-1.05 \mathrm{e} \AA^{-3}$ \\
\hline
\end{tabular}

Special details

Geometry. All esds (except the esd in the dihedral angle between two I.s. planes) are 
estimated using the full covariance matrix. The cell esds are taken into account individually in the estimation of esds in distances, angles and torsion angles; correlations between esds in cell parameters are only used when they are defined by crystal symmetry. An approximate (isotropic) treatment of cell esds is used for estimating esds involving I.s. planes.

Fractional atomic coordinates and isotropic or equivalent isotropic displacement parameters $\left(\AA^{2}\right)$ for (1) $\cdot \mathrm{CH}_{2} \mathrm{Cl}_{2}$

\begin{tabular}{|c|c|c|c|c|c|}
\hline & $x$ & $y$ & $z$ & $U_{\text {iso }}{ }^{*} / U_{\text {eq }}$ & Occ. $(<1)$ \\
\hline Os1 & $0.24602(2)$ & $0.48548(2)$ & $0.15721(2)$ & $0.01357(4)$ & \\
\hline Os2 & $0.23338(2)$ & $0.49256(2)$ & $0.34230(2)$ & $0.01296(4)$ & \\
\hline P1 & $0.25891(4)$ & $0.50855(4)$ & $-0.00045(5)$ & $\begin{array}{l}0.01549 \\
(15)\end{array}$ & \\
\hline P2 & $0.21444(4)$ & $0.53185(4)$ & $0.49394(5)$ & $\begin{array}{l}0.01377 \\
(14)\end{array}$ & \\
\hline 01 & $\begin{array}{l}0.39063 \\
(12)\end{array}$ & $\begin{array}{l}0.41219 \\
(15)\end{array}$ & $\begin{array}{l}0.17787 \\
(16)\end{array}$ & $0.0266(5)$ & \\
\hline $\mathrm{O} 2$ & $\begin{array}{l}0.17142 \\
(14)\end{array}$ & $\begin{array}{l}0.34201 \\
(14)\end{array}$ & $\begin{array}{l}0.13616 \\
(17)\end{array}$ & $0.0276(5)$ & \\
\hline $\mathrm{O} 3$ & $\begin{array}{l}0.36509 \\
(14)\end{array}$ & $\begin{array}{l}0.40392 \\
(17)\end{array}$ & $\begin{array}{l}0.39324 \\
(17)\end{array}$ & $0.0354(6)$ & \\
\hline $\mathrm{O} 4$ & $\begin{array}{l}0.14255 \\
(14)\end{array}$ & $\begin{array}{l}0.35845 \\
(14)\end{array}$ & $\begin{array}{l}0.35453 \\
(16)\end{array}$ & $0.0290(5)$ & \\
\hline O5 & $\begin{array}{l}0.28772 \\
(13)\end{array}$ & $\begin{array}{l}0.59099 \\
(13)\end{array}$ & $\begin{array}{l}0.17664 \\
(14)\end{array}$ & $0.0217(5)$ & \\
\hline $\mathrm{O} 6$ & $\begin{array}{l}0.29035 \\
(13)\end{array}$ & $\begin{array}{l}0.59111 \\
(14)\end{array}$ & $\begin{array}{l}0.32777 \\
(15)\end{array}$ & $0.0253(5)$ & \\
\hline 07 & $\begin{array}{l}0.14672 \\
(11)\end{array}$ & $\begin{array}{l}0.54428 \\
(13)\end{array}$ & $\begin{array}{l}0.15336 \\
(14)\end{array}$ & $0.0211(5)$ & \\
\hline
\end{tabular}




\begin{tabular}{|c|c|c|c|c|}
\hline $\mathrm{O} 8$ & $\begin{array}{l}0.14444 \\
(12)\end{array}$ & $\begin{array}{l}0.55903 \\
(14)\end{array}$ & $\begin{array}{l}0.30353 \\
(14)\end{array}$ & $0.0220(5)$ \\
\hline C1 & $\begin{array}{l}0.33496 \\
(17)\end{array}$ & $\begin{array}{l}0.44046 \\
(18)\end{array}$ & $\begin{array}{l}0.16955 \\
(19)\end{array}$ & $0.0187(6)$ \\
\hline C2 & $\begin{array}{l}0.20033 \\
(16)\end{array}$ & $\begin{array}{l}0.39689 \\
(19)\end{array}$ & $0.1439(2)$ & $0.0204(7)$ \\
\hline C3 & $\begin{array}{l}0.31432 \\
(18)\end{array}$ & $0.4387(2)$ & $0.3731(2)$ & $0.0221(7)$ \\
\hline C4 & $\begin{array}{l}0.17734 \\
(17)\end{array}$ & $\begin{array}{l}0.40972 \\
(19)\end{array}$ & $0.3493(2)$ & $0.0210(7)$ \\
\hline C5 & $\begin{array}{l}0.29965 \\
(17)\end{array}$ & $\begin{array}{l}0.62065 \\
(17)\end{array}$ & $0.2519(2)$ & $0.0218(6)$ \\
\hline C8 & $\begin{array}{l}0.12202 \\
(15)\end{array}$ & $\begin{array}{l}0.57186 \\
(17)\end{array}$ & $0.2239(2)$ & $0.0188(6)$ \\
\hline C10 & $\begin{array}{l}0.29294 \\
(16)\end{array}$ & $\begin{array}{l}0.56904 \\
(16)\end{array}$ & $0.5577(2)$ & $0.0152(6)$ \\
\hline C11 & $\begin{array}{l}0.28798 \\
(17)\end{array}$ & $\begin{array}{l}0.58869 \\
(19)\end{array}$ & $0.6477(2)$ & $0.0206(7)$ \\
\hline $\mathrm{H} 11$ & 0.243680 & 0.581524 & 0.677009 & $0.025^{\star}$ \\
\hline C12 & $\begin{array}{l}0.34607 \\
(17)\end{array}$ & $0.6185(2)$ & $0.6957(2)$ & $0.0214(7)$ \\
\hline $\mathrm{H} 12$ & 0.341304 & 0.631017 & 0.757372 & $0.026^{*}$ \\
\hline $\mathrm{C} 13$ & $\begin{array}{l}0.41184 \\
(16)\end{array}$ & $\begin{array}{l}0.63028 \\
(18)\end{array}$ & $0.6541(2)$ & $0.0190(6)$ \\
\hline C14 & $\begin{array}{l}0.41764 \\
(17)\end{array}$ & $0.6085(2)$ & $0.5653(2)$ & $0.0222(7)$ \\
\hline $\mathrm{H} 14$ & 0.462308 & 0.614585 & 0.536482 & $0.027^{*}$ \\
\hline C15 & $\begin{array}{l}0.35921 \\
(17)\end{array}$ & $\begin{array}{l}0.57797 \\
(19)\end{array}$ & $0.5174(2)$ & $0.0218(7)$ \\
\hline
\end{tabular}




\begin{tabular}{|c|c|c|c|c|}
\hline $\mathrm{H} 15$ & 0.364641 & 0.563058 & 0.456666 & $0.026^{*}$ \\
\hline C16 & $\begin{array}{l}0.47439 \\
(17)\end{array}$ & $0.6672(2)$ & $0.7040(2)$ & $0.0244(7)$ \\
\hline $\mathrm{H} 16 \mathrm{~A}$ & 0.470386 & 0.719545 & 0.695725 & $0.037^{*}$ \\
\hline H16B & 0.520114 & 0.650346 & 0.680050 & $0.037^{*}$ \\
\hline $\mathrm{H} 16 \mathrm{C}$ & 0.473149 & 0.655727 & 0.768528 & $0.037^{*}$ \\
\hline C17 & $\begin{array}{l}0.14668 \\
(16)\end{array}$ & $\begin{array}{l}0.60298 \\
(17)\end{array}$ & $\begin{array}{l}0.49880 \\
(19)\end{array}$ & $0.0154(6)$ \\
\hline C18 & $\begin{array}{l}0.07374 \\
(17)\end{array}$ & $\begin{array}{l}0.58515 \\
(19)\end{array}$ & $0.4842(2)$ & $0.0206(6)$ \\
\hline H18 & 0.060220 & 0.536103 & 0.475853 & $0.025^{*}$ \\
\hline C19 & $\begin{array}{l}0.02107 \\
(17)\end{array}$ & $0.6382(2)$ & $0.4817(2)$ & $0.0227(7)$ \\
\hline $\mathrm{H} 19$ & -0.028264 & 0.624888 & 0.472896 & $0.027^{*}$ \\
\hline C20 & $\begin{array}{l}0.03913 \\
(18)\end{array}$ & $0.7107(2)$ & $0.4918(2)$ & $0.0223(7)$ \\
\hline C21 & $\begin{array}{l}0.11192 \\
(19)\end{array}$ & $\begin{array}{l}0.72843 \\
(19)\end{array}$ & $0.5041(2)$ & $0.0235(7)$ \\
\hline $\mathrm{H} 21$ & 0.125531 & 0.777660 & 0.510092 & $0.028^{*}$ \\
\hline C22 & $\begin{array}{l}0.16513 \\
(17)\end{array}$ & $\begin{array}{l}0.67526 \\
(19)\end{array}$ & $0.5079(2)$ & $0.0204(6)$ \\
\hline H22 & 0.214458 & 0.688563 & 0.516747 & $0.024^{*}$ \\
\hline C23 & $-0.0188(2)$ & $0.7680(2)$ & $0.4890(3)$ & $0.0327(8)$ \\
\hline $\mathrm{H} 23 \mathrm{~A}$ & -0.049023 & 0.762871 & 0.541756 & $0.049^{*}$ \\
\hline H23B & -0.048956 & 0.762628 & 0.433694 & $0.049^{*}$ \\
\hline $\mathrm{H} 23 \mathrm{C}$ & 0.003800 & 0.815822 & 0.489889 & $0.049^{*}$ \\
\hline C24 & 0.18198 & 0.46346 & $0.5708(2)$ & $0.0157(6)$ \\
\hline
\end{tabular}




\begin{tabular}{|l|l|l|l|l|l|}
\hline & $(15)$ & $(18)$ & & \\
\hline C25 & $\begin{array}{l}0.20280 \\
(16)\end{array}$ & $\begin{array}{l}0.39197 \\
(18)\end{array}$ & $0.5619(2)$ & $0.0173(6)$ & \\
\hline H25 & 0.231485 & 0.378490 & 0.512623 & $0.021^{\star}$ & \\
\hline C26 & $\begin{array}{l}0.18271 \\
(16)\end{array}$ & $\begin{array}{l}0.33976 \\
(18)\end{array}$ & $0.6231(2)$ & $0.0180(6)$ & \\
\hline H26 & 0.196732 & 0.291015 & 0.614533 & $0.022^{\star}$ & \\
\hline C27 & $\begin{array}{l}0.14207 \\
(16)\end{array}$ & $\begin{array}{l}0.35819 \\
(19)\end{array}$ & $0.6974(2)$ & $0.0197(6)$ & \\
\hline C28 & $\begin{array}{l}0.12092 \\
(17)\end{array}$ & $\begin{array}{l}0.42973 \\
(19)\end{array}$ & $0.7069(2)$ & $0.0200(6)$ & \\
\hline H28 & 0.093335 & 0.443309 & 0.757079 & $0.024^{\star}$ & \\
\hline C29 & $\begin{array}{l}0.13952 \\
(16)\end{array}$ & $\begin{array}{l}0.48160 \\
(17)\end{array}$ & $0.6441(2)$ & $0.0175(6)$ & \\
\hline H29 & 0.123336 & 0.529865 & 0.650930 & $0.021^{\star}$ & \\
\hline C30 & $0.1246(2)$ & $0.3029(2)$ & $0.7678(2)$ & $0.0283(8)$ & \\
\hline H30A & 0.088624 & 0.268937 & 0.742620 & $0.042^{\star}$ & \\
\hline H30B & 0.105160 & 0.327067 & 0.820513 & $0.042^{\star}$ & \\
\hline H30C & 0.168693 & 0.276563 & 0.786020 & $0.042^{\star}$ & \\
\hline C31 & $\begin{array}{l}0.33106 \\
(17)\end{array}$ & $\begin{array}{l}0.57266 \\
(19)\end{array}$ & $-0.0201(2)$ & $0.0186(6)$ & \\
\hline C32 & 0.32189 & $0.6459(2)$ & $-0.0017(2)$ & $0.0253(7)$ & \\
\hline H32 & 0.276132 & 0.662835 & 0.016272 & $0.030^{\star}$ & \\
\hline C33 & $0.3785(2)$ & $0.6941(2)$ & $-0.0092(3)$ & $0.0325(8)$ & \\
\hline H33 & 0.370747 & 0.743849 & 0.002209 & $0.039^{\star}$ & \\
\hline C34 & $0.6705(2)$ & $-0.0335(2)$ & $0.0291(8)$ & \\
\hline
\end{tabular}




\begin{tabular}{|c|c|c|c|c|}
\hline C35 & $\begin{array}{l}0.45630 \\
(17)\end{array}$ & $0.5974(2)$ & $-0.0506(2)$ & $0.0254(7)$ \\
\hline H35 & 0.502391 & 0.580222 & -0.067077 & $0.030^{*}$ \\
\hline C36 & $\begin{array}{l}0.39947 \\
(16)\end{array}$ & $\begin{array}{l}0.54921 \\
(19)\end{array}$ & $-0.0440(2)$ & $0.0199(6)$ \\
\hline H36 & 0.407204 & 0.499497 & -0.055853 & $0.024^{*}$ \\
\hline C37 & 0.5092 (2) & 0.7229 (3) & $-0.0398(3)$ & $0.0418(10)$ \\
\hline H37A & 0.524938 & 0.738388 & 0.021061 & $0.063^{*}$ \\
\hline H37B & 0.493568 & 0.765050 & -0.075331 & $0.063^{*}$ \\
\hline $\mathrm{H} 37 \mathrm{C}$ & 0.549566 & 0.699297 & -0.069177 & $0.063^{*}$ \\
\hline C38 & $\begin{array}{l}0.28317 \\
(16)\end{array}$ & $\begin{array}{l}0.43215 \\
(18)\end{array}$ & $-0.0719(2)$ & $0.0182(6)$ \\
\hline C39 & $\begin{array}{l}0.29060 \\
(16)\end{array}$ & $\begin{array}{l}0.44399 \\
(19)\end{array}$ & $-0.1646(2)$ & $0.0198(6)$ \\
\hline H39 & 0.281898 & 0.490645 & -0.189198 & $0.024^{*}$ \\
\hline $\mathrm{C} 40$ & $\begin{array}{l}0.31044 \\
(17)\end{array}$ & $0.3884(2)$ & $-0.2204(2)$ & $0.0228(7)$ \\
\hline $\mathrm{H} 40$ & 0.315867 & 0.397591 & -0.282905 & $0.027^{*}$ \\
\hline C41 & $\begin{array}{l}0.32270 \\
(17)\end{array}$ & $0.3190(2)$ & $-0.1872(2)$ & $0.0243(7)$ \\
\hline $\mathrm{C} 42$ & $\begin{array}{l}0.31542 \\
(19)\end{array}$ & $0.3075(2)$ & $-0.0951(2)$ & $0.0268(7)$ \\
\hline $\mathrm{H} 42$ & 0.323761 & 0.260721 & -0.070694 & $0.032^{*}$ \\
\hline C43 & $\begin{array}{l}0.29603 \\
(18)\end{array}$ & $0.3638(2)$ & $-0.0380(2)$ & $0.0239(7)$ \\
\hline $\mathrm{H} 43$ & 0.291638 & 0.354882 & 0.024786 & $0.029^{*}$ \\
\hline C44 & $0.3426(2)$ & $0.2582(2)$ & $-0.2490(3)$ & $0.0353(9)$ \\
\hline
\end{tabular}




\begin{tabular}{|l|l|l|l|l|l|}
\hline H44A & 0.298789 & 0.239353 & -0.279777 & $0.053^{\star}$ & \\
\hline H44B & 0.366032 & 0.219575 & -0.213472 & $0.053^{\star}$ & \\
\hline H44C & 0.375911 & 0.275952 & -0.293940 & $0.053^{\star}$ & \\
\hline C45 & $\begin{array}{l}0.17989 \\
(16)\end{array}$ & $\begin{array}{l}0.54470 \\
(18)\end{array}$ & $-0.0635(2)$ & $0.0176(6)$ & \\
\hline C46 & $\begin{array}{l}0.18411 \\
(17)\end{array}$ & $\begin{array}{l}0.59718 \\
(19)\end{array}$ & $-0.1303(2)$ & $0.0213(7)$ & \\
\hline H46 & 0.229091 & 0.619892 & -0.140735 & $0.026^{\star}$ & \\
\hline C47 & $\begin{array}{l}0.12309 \\
(19)\end{array}$ & $0.6165(2)$ & $-0.1818(2)$ & $0.0247(7)$ & \\
\hline H47 & 0.126739 & 0.652924 & -0.226577 & $0.030^{\star}$ & \\
\hline C48 & $\begin{array}{l}0.05649 \\
(18)\end{array}$ & $0.5835(2)$ & $-0.1689(2)$ & $0.0251(7)$ & \\
\hline C49 & $\begin{array}{l}0.05235 \\
(17)\end{array}$ & $0.5325(2)$ & $-0.1011(2)$ & $0.0234(7)$ & \\
\hline H49 & 0.007187 & 0.510349 & -0.090312 & $0.028^{\star}$ & \\
\hline C50 & $\begin{array}{l}0.11293 \\
(17)\end{array}$ & 0.51307 & $-0.0485(2)$ & $0.0194(6)$ & \\
\hline H50 & 0.108708 & 0.478064 & -0.002159 & $0.023^{\star}$ & \\
\hline C51 & $-0.0085(2)$ & $0.6020(2)$ & $-0.2291(3)$ & $0.0339(9)$ & \\
\hline H51A & -0.044745 & 0.563639 & -0.225184 & $0.051^{*}$ & \\
\hline H51B & 0.006362 & 0.606502 & -0.291712 & $0.051^{\star}$ & \\
\hline H51C & -0.029325 & 0.647717 & -0.209418 & $0.051^{\star}$ & \\
\hline C6 & $0.3157(7)$ & $0.7001(3)$ & $0.2532(14)$ & $0.0286(17)$ & 0.5 \\
\hline H6A & 0.342512 & 0.714118 & 0.199177 & $0.034^{\star}$ & 0.5 \\
\hline H6B & 0.345090 & 0.713084 & 0.307904 & $0.034^{\star}$ & 0.5 \\
\hline C6A & $0.0584(7)$ & $0.6216(7)$ & $0.211(2)$ & $0.0189(16)$ & 0.5 \\
\hline
\end{tabular}




\begin{tabular}{|l|l|l|l|l|l|}
\hline H6AA & 0.018924 & 0.607821 & 0.250609 & $0.023^{\star}$ & 0.5 \\
\hline H6AB & 0.039896 & 0.620471 & 0.147180 & $0.023^{\star}$ & 0.5 \\
\hline C7 & $0.2419(6)$ & $0.7378(6)$ & $0.2532(8)$ & $0.063(2)$ & 0.5 \\
\hline H7A & 0.214154 & 0.725351 & 0.197841 & $0.095^{\star}$ & 0.5 \\
\hline H7B & 0.249011 & 0.790100 & 0.256045 & $0.095^{\star}$ & 0.5 \\
\hline H7C & 0.215462 & 0.721881 & 0.305863 & $0.095^{\star}$ & 0.5 \\
\hline C7A & $0.0886(4)$ & $0.6989(4)$ & $0.2361(5)$ & $0.0257(13)$ & 0.5 \\
\hline H7AA & 0.106583 & 0.698975 & 0.299141 & $0.039^{\star}$ & 0.5 \\
\hline H7AB & 0.049730 & 0.734591 & 0.228465 & $0.039^{\star}$ & 0.5 \\
\hline H7AC & 0.128106 & 0.711250 & 0.196543 & $0.039^{\star}$ & 0.5 \\
\hline C9 & $0.0655(7)$ & $0.6299(7)$ & $0.214(2)$ & $0.021(2)$ & 0.5 \\
\hline H9A & 0.089199 & 0.676991 & 0.210375 & $0.031^{\star}$ & 0.5 \\
\hline H9B & 0.034882 & 0.629016 & 0.266930 & $0.031^{\star}$ & 0.5 \\
\hline H9C & 0.035730 & 0.621372 & 0.159332 & $0.031^{\star}$ & 0.5 \\
\hline C9A & $0.3324(6)$ & $0.6948(3)$ & $0.2543(14)$ & $0.033(2)$ & 0.5 \\
\hline H9AA & 0.333486 & 0.713028 & 0.316480 & $0.050^{\star}$ & 0.5 \\
\hline H9AB & 0.303331 & 0.727181 & 0.215434 & $0.050^{\star}$ & 0.5 \\
\hline H9AC & 0.381832 & 0.692543 & 0.232696 & $0.050^{\star}$ & 0.5 \\
\hline Cl1 & $0.48305(6)$ & $0.58572(8)$ & $0.18071(9)$ & $0.0549(3)$ & \\
\hline Cl2 & $0.53460(8)$ & $0.58296(8)$ & $0.37000(8)$ & $0.0608(3)$ & \\
\hline C1S & $0.5169(2)$ & $0.5330(2)$ & $0.2713(3)$ & $0.0395(9)$ & \\
\hline H1SA & 0.481463 & 0.494723 & 0.284202 & $0.047^{\star}$ & \\
\hline H1SB & 0.562162 & 0.509281 & 0.253386 & $0.047^{\star}$ & \\
\hline & & & & & \\
\hline
\end{tabular}

Atomic displacement parameters $\left(\AA^{2}\right)$ for (1) $\cdot \mathrm{CH}_{2} \mathrm{Cl}_{2}$

\begin{tabular}{|l|l|l|l|l|l}
\hline$U^{11}$ & $U^{22}$ & $U^{\beta 3}$ & $U^{12}$ & $U^{13}$ & $U^{23}$
\end{tabular}




\begin{tabular}{|l|l|l|l|l|l|l|}
\hline Os1 & $\begin{array}{l}0.01404 \\
(7)\end{array}$ & $\begin{array}{l}0.01543 \\
(8)\end{array}$ & $\begin{array}{l}0.01151 \\
(7)\end{array}$ & $\begin{array}{l}0.00011 \\
(5)\end{array}$ & $\begin{array}{l}0.00434 \\
(4)\end{array}$ & $\begin{array}{l}-0.00057 \\
(5)\end{array}$ \\
\hline Os2 & $\begin{array}{l}0.01369 \\
(7)\end{array}$ & $\begin{array}{l}0.01444 \\
(7)\end{array}$ & $\begin{array}{l}0.01095 \\
(7)\end{array}$ & $\begin{array}{l}0.00000 \\
(5)\end{array}$ & $\begin{array}{l}0.00317 \\
(4)\end{array}$ & -0.00072 \\
$(4)$
\end{tabular}




\begin{tabular}{|l|l|l|l|l|l|l|}
\hline C4 & $\begin{array}{l}0.0207 \\
(15)\end{array}$ & $\begin{array}{l}0.0274 \\
(19)\end{array}$ & $\begin{array}{l}0.0150 \\
(15)\end{array}$ & $\begin{array}{l}0.0039 \\
(14)\end{array}$ & $\begin{array}{l}0.0015 \\
(11)\end{array}$ & 0.0001 \\
$(13)$
\end{tabular}




\begin{tabular}{|l|l|l|l|l|l|l|}
\hline & $(17)$ & $(17)$ & $(16)$ & $(14)$ & $(13)$ & $(13)$ \\
\hline C22 & $\begin{array}{l}0.0192 \\
(14)\end{array}$ & $\begin{array}{l}0.0211 \\
(17)\end{array}$ & $\begin{array}{l}0.0211 \\
(16)\end{array}$ & $\begin{array}{l}-0.0012 \\
(13)\end{array}$ & $\begin{array}{l}0.0034 \\
(12)\end{array}$ & 0.0003 \\
$(13)$
\end{tabular}




\begin{tabular}{|c|c|c|c|c|c|c|}
\hline C36 & $\begin{array}{l}0.0191 \\
(14)\end{array}$ & $\begin{array}{l}0.0253 \\
(17)\end{array}$ & $\begin{array}{l}0.0154 \\
(14)\end{array}$ & $\begin{array}{l}0.0031 \\
(13)\end{array}$ & $\begin{array}{l}0.0039 \\
(11)\end{array}$ & $\begin{array}{l}-0.0001 \\
(12)\end{array}$ \\
\hline C37 & $0.035(2)$ & $0.045(3)$ & $0.046(2)$ & $\begin{array}{l}-0.0175 \\
(19)\end{array}$ & $\begin{array}{l}0.0157 \\
(18)\end{array}$ & $-0.008(2)$ \\
\hline C38 & $\begin{array}{l}0.0144 \\
(13)\end{array}$ & $\begin{array}{l}0.0227 \\
(17)\end{array}$ & $\begin{array}{l}0.0175 \\
(15)\end{array}$ & $\begin{array}{l}0.0011 \\
(12)\end{array}$ & $\begin{array}{l}0.0037 \\
(11)\end{array}$ & $\begin{array}{l}-0.0046 \\
(12)\end{array}$ \\
\hline C39 & $\begin{array}{l}0.0176 \\
(14)\end{array}$ & $\begin{array}{l}0.0239 \\
(17)\end{array}$ & $\begin{array}{l}0.0180 \\
(15)\end{array}$ & $\begin{array}{l}0.0022 \\
(12)\end{array}$ & $\begin{array}{l}0.0017 \\
(11)\end{array}$ & $\begin{array}{l}0.0000 \\
(13)\end{array}$ \\
\hline $\mathrm{C} 40$ & $\begin{array}{l}0.0194 \\
(15)\end{array}$ & $0.033(2)$ & $\begin{array}{l}0.0158 \\
(15)\end{array}$ & $\begin{array}{l}0.0035 \\
(14)\end{array}$ & $\begin{array}{l}0.0012 \\
(12)\end{array}$ & $\begin{array}{l}-0.0054 \\
(13)\end{array}$ \\
\hline C41 & $\begin{array}{l}0.0199 \\
(15)\end{array}$ & $\begin{array}{l}0.0294 \\
(19)\end{array}$ & $\begin{array}{l}0.0233 \\
(17)\end{array}$ & $\begin{array}{l}0.0059 \\
(14)\end{array}$ & $\begin{array}{l}-0.0012 \\
(12)\end{array}$ & $\begin{array}{l}-0.0074 \\
(14)\end{array}$ \\
\hline C42 & $\begin{array}{l}0.0335 \\
(18)\end{array}$ & $\begin{array}{l}0.0208 \\
(18)\end{array}$ & $\begin{array}{l}0.0261 \\
(18)\end{array}$ & $\begin{array}{l}0.0065 \\
(14)\end{array}$ & $\begin{array}{l}0.0022 \\
(14)\end{array}$ & $\begin{array}{l}-0.0035 \\
(14)\end{array}$ \\
\hline $\mathrm{C} 43$ & $\begin{array}{l}0.0259 \\
(16)\end{array}$ & $\begin{array}{l}0.0259 \\
(18)\end{array}$ & $\begin{array}{l}0.0202 \\
(16)\end{array}$ & $\begin{array}{l}0.0049 \\
(14)\end{array}$ & $\begin{array}{l}0.0046 \\
(12)\end{array}$ & $\begin{array}{l}0.0000 \\
(14)\end{array}$ \\
\hline C44 & $0.040(2)$ & $0.039(2)$ & $\begin{array}{l}0.0268 \\
(19)\end{array}$ & $\begin{array}{l}0.0196 \\
(18)\end{array}$ & $\begin{array}{l}-0.0029 \\
(15)\end{array}$ & $\begin{array}{l}-0.0118 \\
(16)\end{array}$ \\
\hline C45 & $\begin{array}{l}0.0193 \\
(14)\end{array}$ & $\begin{array}{l}0.0196 \\
(16)\end{array}$ & $\begin{array}{l}0.0143 \\
(14)\end{array}$ & $\begin{array}{l}0.0038 \\
(12)\end{array}$ & $\begin{array}{l}0.0046 \\
(11)\end{array}$ & $\begin{array}{l}-0.0016 \\
(12)\end{array}$ \\
\hline $\mathrm{C} 46$ & $\begin{array}{l}0.0222 \\
(15)\end{array}$ & $\begin{array}{l}0.0220 \\
(17)\end{array}$ & $\begin{array}{l}0.0203 \\
(15)\end{array}$ & $\begin{array}{l}0.0034 \\
(13)\end{array}$ & $\begin{array}{l}0.0080 \\
(12)\end{array}$ & $\begin{array}{l}0.0009 \\
(13)\end{array}$ \\
\hline C47 & $\begin{array}{l}0.0291 \\
(17)\end{array}$ & $\begin{array}{l}0.0248 \\
(18)\end{array}$ & $\begin{array}{l}0.0206 \\
(16)\end{array}$ & $\begin{array}{l}0.0087 \\
(14)\end{array}$ & $\begin{array}{l}0.0062 \\
(13)\end{array}$ & $\begin{array}{l}0.0022 \\
(13)\end{array}$ \\
\hline C48 & $\begin{array}{l}0.0235 \\
(16)\end{array}$ & $\begin{array}{l}0.0309 \\
(19)\end{array}$ & $\begin{array}{l}0.0209 \\
(16)\end{array}$ & $\begin{array}{l}0.0086 \\
(14)\end{array}$ & $\begin{array}{l}0.0005 \\
(13)\end{array}$ & $\begin{array}{l}-0.0056 \\
(14)\end{array}$ \\
\hline C49 & $\begin{array}{l}0.0189 \\
(14)\end{array}$ & $\begin{array}{l}0.0318 \\
(19)\end{array}$ & $\begin{array}{l}0.0198 \\
(15)\end{array}$ & $\begin{array}{l}0.0004 \\
(14)\end{array}$ & $\begin{array}{l}0.0060 \\
(12)\end{array}$ & $\begin{array}{l}-0.0065 \\
(14)\end{array}$ \\
\hline C50 & 0.0202 & 0.0267 & 0.0116 & 0.0024 & 0.0047 & -0.0020 \\
\hline
\end{tabular}




\begin{tabular}{|l|l|l|l|l|l|l|}
\hline & $(14)$ & $(18)$ & $(14)$ & $(13)$ & $(11)$ & $(12)$ \\
\hline C51 & $\begin{array}{l}0.0270 \\
(17)\end{array}$ & $0.045(2)$ & $\begin{array}{l}0.0293 \\
(19)\end{array}$ & $\begin{array}{l}0.0083 \\
(17)\end{array}$ & $\begin{array}{l}-0.0011 \\
(14)\end{array}$ & $\begin{array}{l}0.0026 \\
(17)\end{array}$ \\
\hline C6 & $0.036(4)$ & $0.023(3)$ & $0.027(3)$ & $-0.012(3)$ & $0.005(4)$ & $0.001(2)$ \\
\hline C6A & $0.018(3)$ & $0.021(3)$ & $0.018(3)$ & $0.003(2)$ & $0.001(3)$ & $-0.002(3)$ \\
\hline C7 & $0.071(5)$ & $0.042(4)$ & $0.077(5)$ & $0.008(4)$ & $0.019(5)$ & $-0.001(4)$ \\
\hline C7A & $0.030(3)$ & $0.020(3)$ & $0.027(3)$ & $0.004(2)$ & $0.000(3)$ & $-0.003(3)$ \\
\hline C9 & $0.017(3)$ & $0.026(4)$ & $0.019(4)$ & $0.004(3)$ & $-0.001(3)$ & $-0.002(4)$ \\
\hline C9A & $0.043(5)$ & $0.021(3)$ & $0.036(4)$ & $-0.008(3)$ & $0.020(5)$ & $-0.005(3)$ \\
\hline Cl1 & $0.0407(5)$ & $0.0619(8)$ & $0.0615(7)$ & $0.0047(5)$ & $\begin{array}{l}-0.0063 \\
(5)\end{array}$ & $0.0222(6)$ \\
\hline Cl2 & $0.0862(9)$ & $0.0585(8)$ & $0.0396(6)$ & $\begin{array}{l}-0.0064 \\
(7)\end{array}$ & $0.0283(6)$ & $\begin{array}{l}-0.0143 \\
(5)\end{array}$ \\
\hline C1S & $0.045(2)$ & $0.030(2)$ & $0.044(2)$ & -0.0006 & $\begin{array}{l}0.0034 \\
(18)\end{array}$ & $\begin{array}{l}0.0017 \\
(18)\end{array}$ \\
\hline
\end{tabular}

Geometric parameters (Å, $\stackrel{\circ}{)}$ for (1) $\cdot \mathrm{CH}_{2} \mathrm{Cl}_{2}$

\begin{tabular}{|l|l|l|l|}
\hline Os1—Os2 & $2.7623(2)$ & C30-H30A & 0.9800 \\
\hline Os1—P1 & $2.3937(7)$ & C30-H30B & 0.9800 \\
\hline Os1-O5 & $2.119(2)$ & C30-H30C & 0.9800 \\
\hline Os1-O7 & $2.135(2)$ & C31-C32 & $1.396(5)$ \\
\hline Os1—C1 & $1.848(3)$ & C31-C36 & $1.395(4)$ \\
\hline Os1—C2 & $1.854(4)$ & C32-H32 & 0.9500 \\
\hline Os2-P2 & $2.3981(7)$ & C32-C33 & $1.384(5)$ \\
\hline Os2-O6 & $2.124(2)$ & C33-H33 & 0.9500 \\
\hline Os2-O8 & $2.119(2)$ & C33-C34 & $1.398(5)$ \\
\hline Os2-C3 & $1.844(3)$ & C34-C35 & $1.390(6)$ \\
\hline
\end{tabular}




\begin{tabular}{|l|l|l|l|}
\hline Os2-C4 & $1.858(4)$ & C34-C37 & $1.514(5)$ \\
\hline P1—C31 & $1.818(3)$ & C35-H35 & 0.9500 \\
\hline P1-C38 & $1.833(3)$ & C35-C36 & $1.386(5)$ \\
\hline P1-C45 & $1.834(3)$ & C36-H36 & 0.9500 \\
\hline P2-C10 & $1.839(3)$ & C37-H37A & 0.9800 \\
\hline P2-C17 & $1.823(3)$ & C37-H37B & 0.9800 \\
\hline P2-C24 & $1.820(3)$ & C37-H37C & 0.9800 \\
\hline O1-C1 & $1.158(4)$ & C38-C39 & $1.402(4)$ \\
\hline O2-C2 & $1.153(4)$ & C38-C43 & $1.382(5)$ \\
\hline O3-C3 & $1.169(4)$ & C39-H39 & 0.9500 \\
\hline O4-C4 & $1.152(4)$ & C39-C40 & $1.379(5)$ \\
\hline O5-C5 & $1.255(4)$ & C40-H40 & 0.9500 \\
\hline O6-C5 & $1.268(4)$ & C40-C41 & $1.393(5)$ \\
\hline O7-C8 & $1.263(4)$ & C41-C42 & $1.392(5)$ \\
\hline O8-C8 & $1.259(4)$ & C41-C44 & $1.506(5)$ \\
\hline C5-C6 & $1.502(4)$ & C42-H42 & 0.9500 \\
\hline C5-C9A & $1.502(4)$ & C42-C43 & $1.398(5)$ \\
\hline C8-C6A & $1.502(4)$ & C43-H43 & 0.9500 \\
\hline C8-C9 & $1.502(4)$ & C44-H44A & 0.9800 \\
\hline C10-C11 & $1.388(4)$ & C44-H44B & 0.9800 \\
\hline C10-C15 & $1.392(4)$ & C44-H44C & 0.9800 \\
\hline C11-H11 & 0.9500 & C45-C46 & $1.392(5)$ \\
\hline C11-C12 & $1.382(5)$ & C45-C50 & $1.395(5)$ \\
\hline C12-H12 & 0.9500 & C46-H46 & 0.9500 \\
\hline C12-C13 & C46-C47 & $1.387(5)$ \\
\hline
\end{tabular}




\begin{tabular}{|l|l|l|l|}
\hline C13-C14 & $1.383(5)$ & C47-H47 & 0.9500 \\
\hline C13-C16 & $1.515(4)$ & C47-C48 & $1.395(5)$ \\
\hline C14-H14 & 0.9500 & C48-C49 & $1.384(5)$ \\
\hline C14-C15 & $1.393(5)$ & C48-C51 & $1.510(5)$ \\
\hline C15-H15 & 0.9500 & C49-H49 & 0.9500 \\
\hline C16-H16A & 0.9800 & C49-C50 & $1.390(5)$ \\
\hline C16-H16B & 0.9800 & C50-H50 & 0.9500 \\
\hline C16-H16C & 0.9800 & C51-H51A & 0.9800 \\
\hline C17-C18 & $1.399(4)$ & C51-H51B & 0.9800 \\
\hline C17-C22 & $1.388(5)$ & C51-H51C & 0.9800 \\
\hline C18-H18 & 0.9500 & C6-H6A & 0.9900 \\
\hline C18-C19 & $1.384(5)$ & C6-H6B & 0.9900 \\
\hline C19-H19 & 0.9500 & C6-C7 & $1.533(17)$ \\
\hline C19-C20 & $1.392(5)$ & C6A-H6AA & 0.9900 \\
\hline C20-C21 & $1.392(5)$ & C6A-H6AB & 0.9900 \\
\hline C20-C23 & $1.510(5)$ & C6A-C7A & $1.579(18)$ \\
\hline C21-H21 & 0.9500 & C7-H7A & 0.9800 \\
\hline C21-C22 & $1.393(5)$ & C7-H7B & 0.9800 \\
\hline C22-H22 & 0.9500 & C7-H7C & 0.9800 \\
\hline C23-H23A & 0.9800 & C7A-H7AA & 0.9800 \\
\hline C23-H23B & 0.9800 & C7A-H7AB & 0.9800 \\
\hline C23-H23C & 0.9800 & C7A-H7AC & 0.9800 \\
\hline C24-C25 & $1.388(5)$ & C9-H9A & 0.9800 \\
\hline C24-C29 & $1.404(4)$ & C9-H9B & 0.9800 \\
\hline C25-H25 & 0.9500 & & 0.9800 \\
\hline
\end{tabular}




\begin{tabular}{|l|l|l|l|}
\hline C25-C26 & $1.386(4)$ & C9A-H9AA & 0.9800 \\
\hline C26-H26 & 0.9500 & C9A-H9AB & 0.9800 \\
\hline C26-C27 & $1.396(4)$ & C9A-H9AC & 0.9800 \\
\hline C27-C28 & $1.391(5)$ & Cl1-C1S & $1.758(4)$ \\
\hline C27-C30 & $1.505(5)$ & Cl2-C1S & $1.751(4)$ \\
\hline C28-H28 & 0.9500 & C1S-H1SA & 0.9900 \\
\hline C28-C29 & $1.389(5)$ & C1S-H1SB & 0.9900 \\
\hline C29-H29 & 0.9500 & & 100 \\
\hline P1-Os1-Os2 & $166.94(2)$ & C24-C29-H29 & 119.7 \\
\hline O5-Os1-Os2 & $82.20(6)$ & C28-C29-C24 & $120.7(3)$ \\
\hline O5-Os1-P1 & $85.37(6)$ & C28-C29-H29 & 119.7 \\
\hline O5-Os1-O7 & $80.86(9)$ & C27-C30-H30A & 109.5 \\
\hline O7-Os1-Os2 & $84.24(6)$ & C27-C30-H30B & 109.5 \\
\hline O7-Os1-P1 & $89.86(6)$ & C27-C30-H30C & 109.5 \\
\hline C1-Os1-Os2 & $91.67(9)$ & $\begin{array}{l}\text { H30A-C30- } \\
\text { H30B }\end{array}$ & 109.5 \\
\hline C1-Os1-P1 & $93.36(9)$ & $\begin{array}{l}\text { H30A-C30- } \\
\text { H30C }\end{array}$ & 109.5 \\
\hline C1-Os1-O5 & $94.86(12)$ & H30B-C30- & 109.5 \\
\hline C1-Os1-O7 & $174.43(11)$ & C32-C31-P1 & $120.6(2)$ \\
\hline C1-Os1-C2 & $90.66(14)$ & C32-C31-C36 & $118.0(3)$ \\
\hline C2-Os1-Os2 & $95.37(9)$ & C36-C31-P1 & $121.0(3)$ \\
\hline C2-Os1-P1 & $96.60(10)$ & C31-C32-H32 & 119.5 \\
\hline C2-Os1-O5 & $174.02(11)$ & C33-C32-C31 & $121.0(3)$ \\
\hline
\end{tabular}




\begin{tabular}{|l|l|l|l|}
\hline C2-Os1-O7 & $93.48(12)$ & C33-C32-H32 & 119.5 \\
\hline P2-Os2-Os1 & $164.55(2)$ & C32-C33-H33 & 119.6 \\
\hline O6-Os2-Os1 & $83.16(6)$ & C32-C33-C34 & $120.8(4)$ \\
\hline O6-Os2-P2 & $85.57(6)$ & C34-C33-H33 & 119.6 \\
\hline O8-Os2-Os1 & $81.41(6)$ & C33-C34-C37 & $120.9(4)$ \\
\hline O8-Os2-P2 & $86.54(6)$ & C35-C34-C33 & $118.2(3)$ \\
\hline O8-Os2-O6 & $81.70(10)$ & C35-C34-C37 & $121.0(3)$ \\
\hline C3-Os2-Os1 & $97.08(9)$ & C34-C35-H35 & 119.5 \\
\hline C3-Os2-P2 & $94.43(9)$ & C36-C35-C34 & $121.0(3)$ \\
\hline C3-Os2-O6 & $95.14(13)$ & C36-C35-H35 & 119.5 \\
\hline C3-Os2-O8 & $176.62(13)$ & C31-C36-H36 & 119.5 \\
\hline C3-Os2-C4 & $89.32(15)$ & C35-C36-C31 & $120.9(3)$ \\
\hline C4-Os2-Os1 & $94.74(9)$ & C35-C36-H36 & 119.5 \\
\hline C4-Os2-P2 & $95.67(10)$ & C34-C37-H37A & 109.5 \\
\hline C4-Os2-O6 & $175.27(12)$ & C34-C37-H37B & 109.5 \\
\hline C4-Os2-O8 & $93.80(12)$ & C34-C37-H37C & 109.5 \\
\hline C31-P1-Os1 & $111.82(10)$ & $\begin{array}{l}\text { H37A-C37- } \\
\text { H37B }\end{array}$ & 109.5 \\
\hline C31-P1-C38 & $102.51(14)$ & $\begin{array}{l}\text { H37A-C37- } \\
\text { H37C }\end{array}$ & 109.5 \\
\hline C31-P1-C45 & $104.85(15)$ & $\begin{array}{l}\text { H37B-C37- } \\
\text { H37C }\end{array}$ & 109.5 \\
\hline C38-P1-Os1 & $117.36(11)$ & C39-C38-P1 & $118.6(3)$ \\
\hline C45-P1-Os1 & $117.19(10)$ & C43-C38-P1 & $122.8(2)$ \\
\hline C45-P1-C38 & $101.28(14)$ & C43-C38-C39 & $118.6(3)$ \\
\hline C10-P2-Os2 & $117.15(10)$ & C38-C39-H39 & 119.8 \\
\hline
\end{tabular}




\begin{tabular}{|l|l|l|l|}
\hline C17-P2-Os2 & $112.33(10)$ & C40-C39-C38 & $120.5(3)$ \\
\hline C17-P2-C10 & $103.91(14)$ & C40-C39-H39 & 119.8 \\
\hline C24-P2-Os2 & $115.85(10)$ & C39-C40-H40 & 119.2 \\
\hline C24-P2-C10 & $102.25(13)$ & C39-C40-C41 & $121.5(3)$ \\
\hline C24-P2-C17 & $103.71(14)$ & C41-C40-H40 & 119.2 \\
\hline C5-O5-Os1 & $125.2(2)$ & C40-C41-C44 & $121.2(3)$ \\
\hline C5-O6-Os2 & $123.0(2)$ & C42-C41-C40 & $117.9(3)$ \\
\hline C8-O7-Os1 & $121.36(19)$ & C42-C41-C44 & $121.0(3)$ \\
\hline C8-O8-Os2 & $126.24(19)$ & C41-C42-H42 & 119.5 \\
\hline O1-C1-Os1 & $179.6(3)$ & C41-C42-C43 & $121.0(3)$ \\
\hline O2-C2-Os1 & $179.3(3)$ & C43-C42-H42 & 119.5 \\
\hline O3-C3-Os2 & $179.1(3)$ & C38-C43-C42 & $120.6(3)$ \\
\hline O4-C4-Os2 & $179.3(3)$ & C38-C43-H43 & 119.7 \\
\hline O5-C5-O6 & $124.9(3)$ & C42-C43-H43 & 119.7 \\
\hline O5-C5-C6 & $118.0(8)$ & C41-C44-H44A & 109.5 \\
\hline O5-C5-C9A & $118.7(9)$ & C41-C44-H44B & 109.5 \\
\hline O6-C5-C6 & $116.4(8)$ & C41-C44-H44C & 109.5 \\
\hline O6-C5-C9A & $116.2(9)$ & $\begin{array}{l}\text { H44A-C44- } \\
\text { H44B }\end{array}$ & 109.5 \\
\hline O7-C8-C6A & $116.4(12)$ & $\begin{array}{l}\text { H44A-C44- } \\
\text { H44C }\end{array}$ & 109.5 \\
\hline O7-C8-C9 & $118.8(12)$ & $\begin{array}{l}\text { H44B-C44- } \\
\text { H44C }\end{array}$ & 109.5 \\
\hline O8-C8-O7 & $125.5(3)$ & C46-C45-P1 & $123.6(2)$ \\
\hline O8-C8-C6A & $118.0(12)$ & C46-C45-C50 & $118.6(3)$ \\
\hline O8-C8-C9 & $115.4(11)$ & C50-C45-P1 & $117.5(2)$ \\
\hline
\end{tabular}




\begin{tabular}{|l|l|l|l|}
\hline C11-C10-P2 & $120.9(2)$ & C45-C46-H46 & 119.8 \\
\hline C11-C10-C15 & $118.0(3)$ & C47-C46-C45 & $120.5(3)$ \\
\hline C15-C10-P2 & $121.1(2)$ & C47-C46-H46 & 119.8 \\
\hline C10-C11-H11 & 119.3 & C46-C47-H47 & 119.4 \\
\hline C12-C11-C10 & $121.5(3)$ & C46-C47-C48 & $121.2(3)$ \\
\hline C12-C11-H11 & 119.3 & C48-C47-H47 & 119.4 \\
\hline C11-C12-H12 & 119.7 & C47-C48-C51 & $120.6(3)$ \\
\hline C11-C12-C13 & $120.6(3)$ & C49-C48-C47 & $118.1(3)$ \\
\hline C13-C12-H12 & 119.7 & C49-C48-C51 & $121.3(3)$ \\
\hline C12-C13-C16 & $121.2(3)$ & C48-C49-H49 & 119.3 \\
\hline C14-C13-C12 & $118.0(3)$ & C48-C49-C50 & $121.4(3)$ \\
\hline C14-C13-C16 & $120.8(3)$ & C50-C49-H49 & 119.3 \\
\hline C13-C14-H14 & 119.4 & C45-C50-H50 & 119.8 \\
\hline C13-C14-C15 & $121.2(3)$ & C49-C50-C45 & $120.3(3)$ \\
\hline C15-C14-H14 & 119.4 & C49-C50-H50 & 119.8 \\
\hline C10-C15-C14 & $120.7(3)$ & C48-C51-H51A & 109.5 \\
\hline C10-C15-H15 & 119.7 & C48-C51-H51B & 109.5 \\
\hline C14-C15-H15 & 119.7 & C48-C51-H51C & 109.5 \\
\hline C13-C16-H16A & 109.5 & $\begin{array}{l}\text { H51A-C51- } \\
\text { H51B }\end{array}$ & 109.5 \\
\hline C13-C16-H16B & 109.5 & H51A-C51- & 109.5 \\
\hline C13-C16-H16C & 109.5 & H51C & 109.5 \\
\hline H16A-C16-H16B & 109.5 & H51B-C51- & 110.6 \\
\hline H16A-C16-H16C & 109.5 & C5-C6-H6A & 110.6 \\
\hline
\end{tabular}




\begin{tabular}{|c|c|c|c|}
\hline $\mathrm{H} 16 \mathrm{~B}-\mathrm{C} 16-\mathrm{H} 16 \mathrm{C}$ & 109.5 & $\mathrm{C} 5-\mathrm{C} 6-\mathrm{C} 7$ & $105.7(8)$ \\
\hline $\mathrm{C} 18-\mathrm{C} 17-\mathrm{P} 2$ & $118.9(3)$ & $\mathrm{H} 6 \mathrm{~A}-\mathrm{C} 6-\mathrm{H} 6 \mathrm{~B}$ & 108.7 \\
\hline C22-C17-P2 & $122.3(2)$ & $\mathrm{C} 7-\mathrm{C} 6-\mathrm{H} 6 \mathrm{~A}$ & 110.6 \\
\hline C22-C17-C18 & $118.4(3)$ & $\mathrm{C} 7-\mathrm{C} 6-\mathrm{H} 6 \mathrm{~B}$ & 110.6 \\
\hline $\mathrm{C} 17-\mathrm{C} 18-\mathrm{H} 18$ & 119.6 & $\mathrm{C} 8-\mathrm{C} 6 \mathrm{~A}-\mathrm{H} 6 \mathrm{AA}$ & 110.8 \\
\hline C19-C18-C17 & $120.7(3)$ & $\mathrm{C} 8-\mathrm{C} 6 \mathrm{~A}-\mathrm{H} 6 \mathrm{AB}$ & 110.8 \\
\hline $\mathrm{C} 19-\mathrm{C} 18-\mathrm{H} 18$ & 119.6 & $\mathrm{C} 8-\mathrm{C} 6 \mathrm{~A}-\mathrm{C} 7 \mathrm{~A}$ & $105.0(9)$ \\
\hline $\mathrm{C} 18-\mathrm{C} 19-\mathrm{H} 19$ & 119.4 & $\begin{array}{l}\mathrm{H} 6 \mathrm{AA}-\mathrm{C} 6 \mathrm{~A}- \\
\mathrm{H} 6 \mathrm{AB}\end{array}$ & 108.8 \\
\hline C18-C19-C20 & $121.1(3)$ & $\mathrm{C} 7 \mathrm{~A}-\mathrm{C} 6 \mathrm{~A}-\mathrm{H} 6 \mathrm{AA}$ & 110.8 \\
\hline $\mathrm{C} 20-\mathrm{C} 19-\mathrm{H} 19$ & 119.4 & $\mathrm{C} 7 \mathrm{~A}-\mathrm{C} 6 \mathrm{~A}-\mathrm{H} 6 \mathrm{AB}$ & 110.8 \\
\hline C19-C20-C23 & $120.6(3)$ & $\mathrm{C} 6-\mathrm{C} 7-\mathrm{H} 7 \mathrm{~A}$ & 109.5 \\
\hline C21-C20-C19 & $118.0(3)$ & $\mathrm{C} 6-\mathrm{C} 7-\mathrm{H} 7 \mathrm{~B}$ & 109.5 \\
\hline C21-C20-C23 & $121.3(3)$ & $\mathrm{C} 6-\mathrm{C} 7-\mathrm{H} 7 \mathrm{C}$ & 109.5 \\
\hline $\mathrm{C} 20-\mathrm{C} 21-\mathrm{H} 21$ & 119.4 & $\mathrm{H} 7 \mathrm{~A}-\mathrm{C} 7-\mathrm{H} 7 \mathrm{~B}$ & 109.5 \\
\hline C20-C21-C22 & $121.1(3)$ & $\mathrm{H} 7 \mathrm{~A}-\mathrm{C} 7-\mathrm{H} 7 \mathrm{C}$ & 109.5 \\
\hline $\mathrm{C} 22-\mathrm{C} 21-\mathrm{H} 21$ & 119.4 & $\mathrm{H} 7 \mathrm{~B}-\mathrm{C} 7-\mathrm{H} 7 \mathrm{C}$ & 109.5 \\
\hline C17-C22-C21 & $120.5(3)$ & $\mathrm{C} 6 \mathrm{~A}-\mathrm{C} 7 \mathrm{~A}-\mathrm{H} 7 \mathrm{AA}$ & 109.5 \\
\hline $\mathrm{C} 17-\mathrm{C} 22-\mathrm{H} 22$ & 119.7 & $\mathrm{C} 6 \mathrm{~A}-\mathrm{C} 7 \mathrm{~A}-\mathrm{H} 7 \mathrm{AB}$ & 109.5 \\
\hline $\mathrm{C} 21-\mathrm{C} 22-\mathrm{H} 22$ & 119.7 & $\mathrm{C} 6 \mathrm{~A}-\mathrm{C} 7 \mathrm{~A}-\mathrm{H} 7 \mathrm{AC}$ & 109.5 \\
\hline $\mathrm{C} 20-\mathrm{C} 23-\mathrm{H} 23 \mathrm{~A}$ & 109.5 & $\begin{array}{l}\text { H7AA-C7A- } \\
\text { H7AB }\end{array}$ & 109.5 \\
\hline $\mathrm{C} 20-\mathrm{C} 23-\mathrm{H} 23 \mathrm{~B}$ & 109.5 & $\begin{array}{l}\mathrm{H} 7 \mathrm{AA}-\mathrm{C} 7 \mathrm{~A}- \\
\mathrm{H} 7 \mathrm{AC}\end{array}$ & 109.5 \\
\hline $\mathrm{C} 20-\mathrm{C} 23-\mathrm{H} 23 \mathrm{C}$ & 109.5 & $\begin{array}{l}\mathrm{H} 7 \mathrm{AB}-\mathrm{C} 7 \mathrm{~A}- \\
\mathrm{H} 7 \mathrm{AC}\end{array}$ & 109.5 \\
\hline
\end{tabular}




\begin{tabular}{|c|c|c|c|}
\hline $\mathrm{H} 23 \mathrm{~A}-\mathrm{C} 23-\mathrm{H} 23 \mathrm{~B}$ & 109.5 & $\mathrm{C} 8-\mathrm{C} 9-\mathrm{H} 9 \mathrm{~A}$ & 109.5 \\
\hline $\mathrm{H} 23 \mathrm{~A}-\mathrm{C} 23-\mathrm{H} 23 \mathrm{C}$ & 109.5 & $\mathrm{C} 8-\mathrm{C} 9-\mathrm{H} 9 \mathrm{~B}$ & 109.5 \\
\hline $\mathrm{H} 23 \mathrm{~B}-\mathrm{C} 23-\mathrm{H} 23 \mathrm{C}$ & 109.5 & $\mathrm{C} 8-\mathrm{C} 9-\mathrm{H} 9 \mathrm{C}$ & 109.5 \\
\hline C25-C24-P2 & $120.5(2)$ & $\mathrm{H} 9 \mathrm{~A}-\mathrm{C} 9-\mathrm{H} 9 \mathrm{~B}$ & 109.5 \\
\hline C25-C24-C29 & $117.9(3)$ & $\mathrm{H} 9 \mathrm{~A}-\mathrm{C} 9-\mathrm{H} 9 \mathrm{C}$ & 109.5 \\
\hline C29-C24-P2 & $121.5(2)$ & $\mathrm{H} 9 \mathrm{~B}-\mathrm{C} 9-\mathrm{H} 9 \mathrm{C}$ & 109.5 \\
\hline C24-C25-H25 & 119.2 & C5-C9A-H9AA & 109.5 \\
\hline C26-C25-C24 & $121.5(3)$ & C5-C9A-H9AB & 109.5 \\
\hline $\mathrm{C} 26-\mathrm{C} 25-\mathrm{H} 25$ & 119.2 & C5-C9A-H9AC & 109.5 \\
\hline $\mathrm{C} 25-\mathrm{C} 26-\mathrm{H} 26$ & 119.7 & $\begin{array}{l}\mathrm{H} 9 \mathrm{AA}-\mathrm{C} 9 \mathrm{~A}- \\
\mathrm{H} 9 \mathrm{AB}\end{array}$ & 109.5 \\
\hline $\mathrm{C} 25-\mathrm{C} 26-\mathrm{C} 27$ & $120.6(3)$ & $\begin{array}{l}\mathrm{H} 9 \mathrm{AA}-\mathrm{C} 9 \mathrm{~A}- \\
\mathrm{H} 9 \mathrm{AC}\end{array}$ & 109.5 \\
\hline C27-C26-H26 & 119.7 & $\begin{array}{l}\text { H9AB-C9A- } \\
\text { H9AC }\end{array}$ & 109.5 \\
\hline C26-C27-C30 & $120.9(3)$ & $\mathrm{Cl} 1-\mathrm{C} 1 \mathrm{~S}-\mathrm{H} 1 \mathrm{SA}$ & 108.9 \\
\hline C28-C27-C26 & $118.3(3)$ & $\mathrm{Cl} 1-\mathrm{C} 1 \mathrm{~S}-\mathrm{H} 1 \mathrm{SB}$ & 108.9 \\
\hline C28-C27-C30 & $120.8(3)$ & $\mathrm{Cl} 2-\mathrm{C} 1 \mathrm{~S}-\mathrm{Cl} 1$ & $113.2(3)$ \\
\hline C27-C28-H28 & 119.5 & $\mathrm{Cl} 2-\mathrm{C} 1 \mathrm{~S}-\mathrm{H} 1 \mathrm{SA}$ & 108.9 \\
\hline C29-C28-C27 & $121.0(3)$ & $\mathrm{Cl} 2-\mathrm{C} 1 \mathrm{~S}-\mathrm{H} 1 \mathrm{SB}$ & 108.9 \\
\hline $\mathrm{C} 29-\mathrm{C} 28-\mathrm{H} 28$ & 119.5 & $\begin{array}{l}\mathrm{H} 1 \mathrm{SA}-\mathrm{C} 1 \mathrm{~S}- \\
\mathrm{H} 1 \mathrm{SB}\end{array}$ & 107.7 \\
\hline $\begin{array}{l}\text { Os1-P1-C31- } \\
\text { C32 }\end{array}$ & $-73.8(3)$ & $\begin{array}{l}\text { C17-C18-C19- } \\
\text { C20 }\end{array}$ & $1.3(5)$ \\
\hline $\begin{array}{l}\text { Os1-P1-C31- } \\
\text { C36 }\end{array}$ & $98.8(2)$ & $\begin{array}{l}\mathrm{C} 18-\mathrm{C} 17-\mathrm{C} 22- \\
\mathrm{C} 21\end{array}$ & $1.2(5)$ \\
\hline
\end{tabular}




\begin{tabular}{|c|c|c|c|}
\hline $\begin{array}{l}\text { Os1-P1-C38- } \\
\text { C39 }\end{array}$ & $179.6(2)$ & $\begin{array}{l}\mathrm{C} 18-\mathrm{C} 19-\mathrm{C} 20- \\
\mathrm{C} 21\end{array}$ & $0.3(5)$ \\
\hline $\begin{array}{l}\text { Os1-P1-C38- } \\
\text { C43 }\end{array}$ & $-1.9(3)$ & $\begin{array}{l}\mathrm{C} 18-\mathrm{C} 19-\mathrm{C} 20- \\
\mathrm{C} 23\end{array}$ & $-180.0(3)$ \\
\hline $\begin{array}{l}\text { Os1-P1-C45- } \\
\text { C46 }\end{array}$ & $141.0(2)$ & $\begin{array}{l}\mathrm{C} 19-\mathrm{C} 20-\mathrm{C} 21- \\
\mathrm{C} 22\end{array}$ & $-1.2(5)$ \\
\hline $\begin{array}{l}\text { Os1-P1-C45- } \\
\text { C50 }\end{array}$ & $-44.7(3)$ & $\begin{array}{l}\mathrm{C} 20-\mathrm{C} 21-\mathrm{C} 22- \\
\mathrm{C} 17\end{array}$ & $0.4(5)$ \\
\hline Os1-O5-C5-O6 & $2.0(5)$ & $\begin{array}{l}\text { C22-C17-C18- } \\
\text { C19 }\end{array}$ & $-2.0(5)$ \\
\hline Os1-O5-C5-C6 & $-168.6(6)$ & $\begin{array}{l}\mathrm{C} 23-\mathrm{C} 20-\mathrm{C} 21- \\
\mathrm{C} 22\end{array}$ & $179.1(3)$ \\
\hline $\begin{array}{l}\text { Os1-O5-C5- } \\
\text { C9A }\end{array}$ & $177.4(6)$ & $\begin{array}{l}\text { C24-P2-C10- } \\
\text { C11 }\end{array}$ & $-48.0(3)$ \\
\hline Os1-O7-C8-O8 & $-12.2(5)$ & $\begin{array}{l}\text { C24-P2-C10- } \\
\text { C15 }\end{array}$ & $131.6(3)$ \\
\hline $\begin{array}{l}\text { Os1-O7-C8- } \\
\text { C6A }\end{array}$ & $170.6(8)$ & $\begin{array}{l}\text { C24-P2-C17- } \\
\text { C18 }\end{array}$ & $-52.3(3)$ \\
\hline Os1-O7-C8-C9 & $162.1(8)$ & $\begin{array}{l}\text { C24-P2-C17- } \\
\text { C22 }\end{array}$ & $134.1(3)$ \\
\hline $\begin{array}{l}\text { Os2-P2-C10- } \\
\text { C11 }\end{array}$ & $-175.8(2)$ & $\begin{array}{l}\text { C24-C25-C26- } \\
\text { C27 }\end{array}$ & $-1.6(5)$ \\
\hline $\begin{array}{l}\text { Os2-P2-C10- } \\
\text { C15 }\end{array}$ & $3.9(3)$ & $\begin{array}{l}\text { C25-C24-C29- } \\
\text { C28 }\end{array}$ & $1.8(4)$ \\
\hline $\begin{array}{l}\text { Os2-P2-C17- } \\
\text { C18 }\end{array}$ & $73.6(2)$ & $\begin{array}{l}\text { C25-C26-C27- } \\
\text { C28 }\end{array}$ & $1.5(5)$ \\
\hline $\begin{array}{l}\text { Os2-P2-C17- } \\
\text { C22 }\end{array}$ & $-100.1(3)$ & $\begin{array}{l}\text { C25-C26-C27- } \\
\text { C30 }\end{array}$ & $-175.6(3)$ \\
\hline Os2-P2-C24- & $33.0(3)$ & C26-C27-C28- & $0.2(5)$ \\
\hline
\end{tabular}




\begin{tabular}{|c|c|c|c|}
\hline C25 & & C29 & \\
\hline $\begin{array}{l}\text { Os2-P2-C24- } \\
\text { C29 }\end{array}$ & $-151.4(2)$ & $\begin{array}{l}\text { C27-C28-C29- } \\
\text { C24 }\end{array}$ & $-1.8(5)$ \\
\hline Os2-O6-C5-O5 & $-12.3(5)$ & $\begin{array}{l}\mathrm{C} 29-\mathrm{C} 24-\mathrm{C} 25- \\
\mathrm{C} 26\end{array}$ & $-0.1(4)$ \\
\hline Os2-O6-C5-C6 & $158.4(6)$ & $\begin{array}{l}\mathrm{C} 30-\mathrm{C} 27-\mathrm{C} 28- \\
\mathrm{C} 29\end{array}$ & $177.3(3)$ \\
\hline $\begin{array}{l}\text { Os2-O6-C5- } \\
\text { C9A }\end{array}$ & $172.2(6)$ & $\begin{array}{l}\mathrm{C} 31-\mathrm{P} 1-\mathrm{C} 38- \\
\mathrm{C} 39\end{array}$ & $-57.5(3)$ \\
\hline $\mathrm{Os} 2-\mathrm{O} 8-\mathrm{C} 8-\mathrm{O} 7$ & $3.7(5)$ & $\begin{array}{l}\text { C31-P1-C38- } \\
\text { C43 }\end{array}$ & $121.0(3)$ \\
\hline $\begin{array}{l}\text { Os2-O8-C8- } \\
\text { C6A }\end{array}$ & $-179.1(8)$ & $\begin{array}{l}\mathrm{C} 31-\mathrm{P} 1-\mathrm{C} 45- \\
\mathrm{C} 46\end{array}$ & $16.3(3)$ \\
\hline Os2-O8-C8-C9 & $-170.7(8)$ & $\begin{array}{l}\text { C31-P1-C45- } \\
\text { C50 }\end{array}$ & $-169.4(2)$ \\
\hline $\begin{array}{l}\mathrm{P} 1-\mathrm{C} 31-\mathrm{C} 32- \\
\mathrm{C} 33\end{array}$ & $174.5(3)$ & $\begin{array}{l}\text { C31-C32-C33- } \\
\text { C34 }\end{array}$ & $-1.5(6)$ \\
\hline $\begin{array}{l}\mathrm{P} 1-\mathrm{C} 31-\mathrm{C} 36- \\
\mathrm{C} 35\end{array}$ & $-173.8(2)$ & $\begin{array}{l}\text { C32-C31-C36- } \\
\text { C35 }\end{array}$ & $-1.0(5)$ \\
\hline $\begin{array}{l}\mathrm{P} 1-\mathrm{C} 38-\mathrm{C} 39- \\
\mathrm{C} 40\end{array}$ & $178.5(2)$ & $\begin{array}{l}\text { C32-C33-C34- } \\
\text { C35 }\end{array}$ & $0.6(6)$ \\
\hline $\begin{array}{l}\mathrm{P} 1-\mathrm{C} 38-\mathrm{C} 43- \\
\mathrm{C} 42\end{array}$ & $-179.0(3)$ & $\begin{array}{l}\text { C32-C33-C34- } \\
\text { C37 }\end{array}$ & $-178.7(4)$ \\
\hline $\begin{array}{l}\mathrm{P} 1-\mathrm{C} 45-\mathrm{C} 46- \\
\mathrm{C} 47\end{array}$ & $173.2(3)$ & $\begin{array}{l}\text { C33-C34-C35- } \\
\text { C36 }\end{array}$ & $0.1(5)$ \\
\hline $\begin{array}{l}\mathrm{P} 1-\mathrm{C} 45-\mathrm{C} 50- \\
\mathrm{C} 49\end{array}$ & $-173.0(3)$ & $\begin{array}{l}\text { C34-C35-C36- } \\
\text { C31 }\end{array}$ & $0.1(5)$ \\
\hline $\begin{array}{l}\mathrm{P} 2-\mathrm{C} 10-\mathrm{C} 11- \\
\mathrm{C} 12\end{array}$ & $-178.3(3)$ & $\begin{array}{l}\text { C36-C31-C32- } \\
\text { C33 }\end{array}$ & $1.7(5)$ \\
\hline
\end{tabular}




\begin{tabular}{|c|c|c|c|}
\hline $\begin{array}{l}\mathrm{P} 2-\mathrm{C} 10-\mathrm{C} 15- \\
\mathrm{C} 14\end{array}$ & $177.7(3)$ & $\begin{array}{l}\text { C37-C34-C35- } \\
\text { C36 }\end{array}$ & $179.3(3)$ \\
\hline $\begin{array}{l}\mathrm{P} 2-\mathrm{C} 17-\mathrm{C} 18- \\
\mathrm{C} 19\end{array}$ & $-175.9(2)$ & $\begin{array}{l}\text { C38-P1-C31- } \\
\text { C32 }\end{array}$ & $159.6(3)$ \\
\hline $\begin{array}{l}\text { P2-C17-C22- } \\
\text { C21 }\end{array}$ & $174.9(2)$ & $\begin{array}{l}\text { C38-P1-C31- } \\
\text { C36 }\end{array}$ & $-27.8(3)$ \\
\hline $\begin{array}{l}\text { P2-C24-C25- } \\
\text { C26 }\end{array}$ & $175.6(2)$ & $\begin{array}{l}\text { C38-P1-C45- } \\
\text { C46 }\end{array}$ & $-90.0(3)$ \\
\hline $\begin{array}{l}\text { P2-C24-C29- } \\
\text { C28 }\end{array}$ & $-173.9(2)$ & $\begin{array}{l}\text { C38-P1-C45- } \\
\text { C50 }\end{array}$ & $84.3(3)$ \\
\hline $\mathrm{O} 5-\mathrm{C} 5-\mathrm{C} 6-\mathrm{C} 7$ & $86.2(12)$ & $\begin{array}{l}\text { C38-C39-C40- } \\
\text { C41 }\end{array}$ & $0.9(5)$ \\
\hline $\mathrm{O} 6-\mathrm{C} 5-\mathrm{C} 6-\mathrm{C} 7$ & $-85.2(12)$ & $\begin{array}{l}\text { C39-C38-C43- } \\
\text { C42 }\end{array}$ & $-0.5(5)$ \\
\hline $\begin{array}{l}\mathrm{O} 7-\mathrm{C} 8-\mathrm{C} 6 \mathrm{~A}- \\
\mathrm{C} 7 \mathrm{~A}\end{array}$ & $-111.4(13)$ & $\begin{array}{l}\text { C39-C40-C41- } \\
\text { C42 }\end{array}$ & $-1.0(5)$ \\
\hline $\begin{array}{l}\mathrm{O} 8-\mathrm{C} 8-\mathrm{C} 6 \mathrm{~A}- \\
\mathrm{C} 7 \mathrm{~A}\end{array}$ & $71.2(17)$ & $\begin{array}{l}\text { C39-C40-C41- } \\
\text { C44 }\end{array}$ & $178.5(3)$ \\
\hline $\begin{array}{l}\mathrm{C} 10-\mathrm{P} 2-\mathrm{C} 17- \\
\mathrm{C} 18\end{array}$ & $-158.9(2)$ & $\begin{array}{l}\text { C40-C41-C42- } \\
\text { C43 }\end{array}$ & $0.4(5)$ \\
\hline $\begin{array}{l}\text { C10-P2-C17- } \\
\text { C22 }\end{array}$ & $27.5(3)$ & $\begin{array}{l}\text { C41-C42-C43- } \\
\text { C38 }\end{array}$ & $0.4(5)$ \\
\hline $\begin{array}{l}\text { C10-P2-C24- } \\
\text { C25 }\end{array}$ & $-95.6(3)$ & $\begin{array}{l}\mathrm{C} 43-\mathrm{C} 38-\mathrm{C} 39- \\
\mathrm{C} 40\end{array}$ & $-0.1(5)$ \\
\hline $\begin{array}{l}\text { C10-P2-C24- } \\
\text { C29 }\end{array}$ & $80.0(3)$ & $\begin{array}{l}\text { C44-C41-C42- } \\
\text { C43 }\end{array}$ & $-179.1(3)$ \\
\hline $\begin{array}{l}\mathrm{C} 10-\mathrm{C} 11-\mathrm{C} 12- \\
\mathrm{C} 13\end{array}$ & $0.6(5)$ & $\begin{array}{l}\text { C45-P1-C31- } \\
\text { C32 }\end{array}$ & $54.2(3)$ \\
\hline $\mathrm{C} 11-\mathrm{C} 10-\mathrm{C} 15-$ & $-2.6(5)$ & C45-P1-C31- & $-133.2(3)$ \\
\hline
\end{tabular}




\begin{tabular}{|l|l|l|l|}
\hline $\mathrm{C} 14$ & & $\mathrm{C} 36$ & \\
\hline $\begin{array}{l}\mathrm{C} 11-\mathrm{C} 12-\mathrm{C} 13- \\
\mathrm{C} 14\end{array}$ & $-2.7(5)$ & $\begin{array}{l}\mathrm{C} 45-\mathrm{P} 1-\mathrm{C} 38- \\
\mathrm{C} 39\end{array}$ & $50.7(3)$ \\
\hline $\begin{array}{l}\mathrm{C} 11-\mathrm{C} 12-\mathrm{C} 13- \\
\mathrm{C} 16\end{array}$ & $176.0(3)$ & $\begin{array}{l}\mathrm{C} 45-\mathrm{P} 1-\mathrm{C} 38- \\
\mathrm{C} 43\end{array}$ & $-130.8(3)$ \\
\hline $\begin{array}{l}\mathrm{C} 12-\mathrm{C} 13-\mathrm{C} 14- \\
\mathrm{C} 15\end{array}$ & $2.1(5)$ & $\begin{array}{l}\mathrm{C} 45-\mathrm{C} 46-\mathrm{C} 47- \\
\mathrm{C} 48\end{array}$ & $-0.9(5)$ \\
\hline $\begin{array}{l}\mathrm{C} 13-\mathrm{C} 14-\mathrm{C} 15- \\
\mathrm{C} 10\end{array}$ & $0.6(6)$ & $\begin{array}{l}\mathrm{C} 46-\mathrm{C} 45-\mathrm{C} 50- \\
\mathrm{C} 49\end{array}$ & $1.6(5)$ \\
\hline $\begin{array}{l}\mathrm{C} 15-\mathrm{C} 10-\mathrm{C} 11- \\
\mathrm{C} 12\end{array}$ & $2.1(5)$ & $\begin{array}{l}\mathrm{C} 46-\mathrm{C} 47-\mathrm{C} 48- \\
\mathrm{C} 49\end{array}$ & $2.2(5)$ \\
\hline $\begin{array}{l}\mathrm{C} 16-\mathrm{C} 13-\mathrm{C} 14- \\
\mathrm{C} 15\end{array}$ & $-176.6(3)$ & $\begin{array}{l}\mathrm{C} 46-\mathrm{C} 47-\mathrm{C} 48- \\
\mathrm{C} 51\end{array}$ & $-176.4(3)$ \\
\hline $\begin{array}{l}\mathrm{C} 17-\mathrm{P} 2-\mathrm{C} 10- \\
\mathrm{C} 11\end{array}$ & $59.7(3)$ & $\begin{array}{l}\mathrm{C} 47-\mathrm{C} 48-\mathrm{C} 49- \\
\mathrm{C} 50\end{array}$ & $-1.6(5)$ \\
\hline $\begin{array}{l}\mathrm{C} 17-\mathrm{P} 2-\mathrm{C} 10- \\
\mathrm{C} 15\end{array}$ & $-120.7(3)$ & $\begin{array}{l}\mathrm{C} 48-\mathrm{C} 49-\mathrm{C} 50- \\
\mathrm{C} 45\end{array}$ & $-0.3(5)$ \\
\hline $\begin{array}{l}\mathrm{C} 17-\mathrm{P} 2-\mathrm{C} 24- \\
\mathrm{C} 25\end{array}$ & $156.6(2)$ & $\begin{array}{l}\mathrm{C} 50-\mathrm{C} 45-\mathrm{C} 46- \\
\mathrm{C} 47\end{array}$ & $-1.1(5)$ \\
\hline $\begin{array}{l}\mathrm{C} 17-\mathrm{P} 2-\mathrm{C} 24- \\
\mathrm{C} 29\end{array}$ & $-27.9(3)$ & $\begin{array}{l}\mathrm{C} 51-\mathrm{C} 48-\mathrm{C} 49- \\
\mathrm{C} 50\end{array}$ & $176.9(3)$ \\
\hline
\end{tabular}

(2)

Crystal data

\begin{tabular}{|c|c|}
\hline $\mathrm{C}_{10} \mathrm{H}_{6} \mathrm{O}_{10} \mathrm{Os}_{2}$ & $F(000)=1192$ \\
\hline$M_{r}=666.55$ & $D_{\mathrm{x}}=2.995 \mathrm{Mg} \mathrm{m}^{-3}$ \\
\hline Monoclinic, $P 2{ }_{1} / n$ & Mo $K \alpha$ radiation, $\lambda=0.71073 \AA$ \\
\hline$a=7.6949(5) \AA$ & Cell parameters from 9606 reflections \\
\hline
\end{tabular}




\begin{tabular}{|l|l|}
\hline$b=14.3612(10) \AA$ & $\theta=2.8-33.1^{\circ}$ \\
\hline$c=13.8623(9) \AA$ & $\mu=17.22 \mathrm{~mm}^{-1}$ \\
\hline$\beta=105.202(1)^{\circ}$ & $T=200 \mathrm{~K}$ \\
\hline$V=1478.29(17) \AA^{3}$ & Block, colorless \\
\hline$Z=4$ & $0.19 \times 0.12 \times 0.07 \mathrm{~mm}$ \\
\hline
\end{tabular}

Data collection

\begin{tabular}{|l|l|}
\hline Bruker APEXII CCD diffractometer & 3003 reflections with $I>2 \mathrm{~s}(I)$ \\
\hline$\phi$ and $\omega$ scans & $R_{\text {int }}=0.046$ \\
\hline $\begin{array}{l}\text { Absorption correction: multi-scan } \\
\text { SADABS (Bruker, 2008) }\end{array}$ & $\theta_{\max }=27.1^{\circ}, \theta_{\min }=2.1^{\circ}$ \\
\hline$T_{\min }=0.295, T_{\max }=0.747$ & $h=-9 \rightarrow 9$ \\
\hline 14002 measured reflections & $k=-18 \rightarrow 18$ \\
\hline 3252 independent reflections & $I=-17 \rightarrow 17$ \\
\hline
\end{tabular}

\section{Refinement}

\begin{tabular}{|l|l|}
\hline Refinement on $F^{2}$ & $\begin{array}{l}\text { Hydrogen site location: inferred from } \\
\text { neighbouring sites } \\
\text { H-atom parameters constrained }\end{array}$ \\
\hline$R\left[F^{2}>2 \sigma\left(F^{2}\right)\right]=0.023$ & $\begin{array}{l}w=1 /\left[\sigma^{2}\left(F_{0}^{2}\right)+(0.0082 P)^{2}+2.0911 P\right] \\
\text { where } P=\left(F_{0}^{2}+2 F_{c}^{2}\right) / 3\end{array}$ \\
\hline$w R\left(F^{2}\right)=0.055$ & $(\Delta / \sigma)_{\max }=0.001$ \\
\hline$S=1.09$ & $\Delta\rangle_{\max }=1.80$ e $\AA^{-3}$ \\
\hline 3252 reflections & $\Delta\rangle_{\min }=-1.33$ e $\AA^{-3}$ \\
\hline 202 parameters & $\begin{array}{l}\text { Extinction correction: } S H E L X L 2018 / 3 \\
(\text { Sheldrick 2018), }\end{array}$ \\
\hline
\end{tabular}




\begin{tabular}{|l|l|}
\hline & $\mathrm{Fc}^{*}=\mathrm{kFc}\left[1+0.001 \mathrm{xc}^{2} \lambda^{3} / \sin (2 \theta)\right]^{-1 / 4}$ \\
\hline 0 restraints & Extinction coefficient: $0.00198(9)$ \\
\hline
\end{tabular}

Special details

Geometry. All esds (except the esd in the dihedral angle between two I.s. planes) are estimated using the full covariance matrix. The cell esds are taken into account individually in the estimation of esds in distances, angles and torsion angles; correlations between esds in cell parameters are only used when they are defined by crystal symmetry. An approximate (isotropic) treatment of cell esds is used for estimating esds involving I.s. planes.

Fractional atomic coordinates and isotropic or equivalent isotropic displacement parameters $\left(\AA^{2}\right)$ for (2)

\begin{tabular}{|l|l|l|l|l|}
\hline & $x$ & $y$ & $z$ & $U_{\text {iso }} / U_{\text {eq }}$ \\
\hline Os1 & $0.42547(2)$ & $0.22664(2)$ & $0.05236(2)$ & $0.01655(7)$ \\
\hline Os2 & $0.77231(2)$ & $0.21800(2)$ & $0.17010(2)$ & $0.01619(7)$ \\
\hline O8 & $0.6745(4)$ & $0.3123(2)$ & $0.2608(2)$ & $0.0223(7)$ \\
\hline O9 & $0.5294(4)$ & $0.3337(2)$ & $-0.0211(2)$ & $0.0239(7)$ \\
\hline O7 & $0.4059(4)$ & $0.3352(2)$ & $0.1523(2)$ & $0.0233(7)$ \\
\hline O10 & $0.8002(4)$ & $0.3386(2)$ & $0.0881(2)$ & $0.0253(7)$ \\
\hline O3 & $0.0418(5)$ & $0.2846(3)$ & $-0.0685(3)$ & $0.0424(10)$ \\
\hline O2 & $0.4741(6)$ & $0.0738(3)$ & $-0.0875(3)$ & $0.0430(10)$ \\
\hline O4 & $0.8856(6)$ & $0.0922(3)$ & $0.0236(3)$ & $0.0391(9)$ \\
\hline C7 & $0.5235(6)$ & $0.3522(3)$ & $0.2335(3)$ & $0.0190(9)$ \\
\hline O5 & $0.7127(6)$ & $0.0534(3)$ & $0.2927(3)$ & $0.0417(9)$ \\
\hline O1 & $0.3004(6)$ & $0.0859(3)$ & $0.1800(3)$ & $0.0462(10)$ \\
\hline O6 & $1.1610(5)$ & $0.2535(4)$ & $0.2995(3)$ & $0.0459(10)$ \\
\hline
\end{tabular}




\begin{tabular}{|l|l|l|l|l|}
\hline C4 & $0.8448(6)$ & $0.1390(3)$ & $0.0800(3)$ & $0.0262(10)$ \\
\hline C1 & $0.3453(6)$ & $0.1378(3)$ & $0.1300(4)$ & $0.0271(10)$ \\
\hline C5 & $0.7343(6)$ & $0.1147(3)$ & $0.2464(3)$ & $0.0243(10)$ \\
\hline C8 & $0.4787(7)$ & $0.4249(3)$ & $0.2994(3)$ & $0.0288(10)$ \\
\hline H8A & 0.579579 & 0.432174 & 0.358999 & $0.043^{\star}$ \\
\hline H8B & 0.370674 & 0.406277 & 0.319369 & $0.043^{\star}$ \\
\hline H8C & 0.456292 & 0.484157 & 0.263170 & $0.043^{\star}$ \\
\hline C9 & $0.6840(6)$ & $0.3691(3)$ & $0.0140(3)$ & $0.0211(9)$ \\
\hline C2 & $0.4554(7)$ & $0.1320(3)$ & $-0.0357(3)$ & $0.0269(10)$ \\
\hline C3 & $0.1837(7)$ & $0.2639(3)$ & $-0.0252(4)$ & $0.0260(10)$ \\
\hline C6 & $1.0190(7)$ & $0.2415(4)$ & $0.2537(4)$ & $0.0263(10)$ \\
\hline C10 & $0.7308(8)$ & $0.4540(4)$ & $-0.0362(4)$ & $0.0350(12)$ \\
\hline H10A & 0.830534 & 0.486855 & 0.009782 & $0.053^{\star}$ \\
\hline H10B & 0.625795 & 0.495203 & -0.055015 & $0.053^{*}$ \\
\hline H10C & 0.766953 & 0.435746 & -0.096241 & $0.053^{*}$ \\
\hline & & & & \\
\hline
\end{tabular}

Atomic displacement parameters $\left(\AA^{2}\right)$ for (2)

\begin{tabular}{|l|l|l|l|l|l|l|}
\hline & $U^{11}$ & $U^{22}$ & $U^{\beta 3}$ & $U^{12}$ & $U^{13}$ & $U^{23}$ \\
\hline Os1 & $\begin{array}{l}0.01386 \\
(11)\end{array}$ & $\begin{array}{l}0.01834 \\
(11)\end{array}$ & $\begin{array}{l}0.01733 \\
(10)\end{array}$ & $\begin{array}{l}0.00051 \\
(6)\end{array}$ & $\begin{array}{l}0.00388 \\
(7)\end{array}$ & $\begin{array}{l}-0.00074 \\
(5)\end{array}$ \\
\hline Os2 & $\begin{array}{l}0.01358 \\
(11)\end{array}$ & $\begin{array}{l}0.01921 \\
(11)\end{array}$ & $\begin{array}{l}0.01643 \\
(10)\end{array}$ & $\begin{array}{l}0.00390 \\
(6)\end{array}$ & $\begin{array}{l}0.00506 \\
(7)\end{array}$ & $\begin{array}{l}0.00252 \\
(5)\end{array}$ \\
\hline O8 & 0.0225 & 0.0263 & 0.0179 & 0.0063 & 0.0052 & -0.0054 \\
& $(17)$ & $(16)$ & $(14)$ & $(13)$ & $(12)$ & $(12)$ \\
\hline O9 & 0.0249 & 0.0267 & 0.0201 & -0.0001 & 0.0058 & 0.0088 \\
\hline O7 & $(17)$ & $(17)$ & $(14)$ & $(14)$ & $(13)$ & $(12)$ \\
\hline
\end{tabular}




\begin{tabular}{|c|c|c|c|c|c|c|}
\hline & (17) & (16) & (14) & (13) & (13) & (12) \\
\hline 010 & $\begin{array}{l}0.0228 \\
(17)\end{array}$ & $\begin{array}{l}0.0235 \\
(17)\end{array}$ & $\begin{array}{l}0.0281 \\
(16)\end{array}$ & $\begin{array}{l}-0.0032 \\
(13)\end{array}$ & $\begin{array}{l}0.0042 \\
(13)\end{array}$ & $\begin{array}{l}0.0067 \\
(13)\end{array}$ \\
\hline $\mathrm{O} 3$ & $0.020(2)$ & $0.059(3)$ & $0.043(2)$ & $\begin{array}{l}0.0106 \\
(17)\end{array}$ & $\begin{array}{l}-0.0002 \\
(18)\end{array}$ & $\begin{array}{l}-0.0021 \\
(17)\end{array}$ \\
\hline $\mathrm{O} 2$ & $0.051(2)$ & $0.038(2)$ & $\begin{array}{l}0.0347 \\
(19)\end{array}$ & $\begin{array}{l}0.0136 \\
(19)\end{array}$ & $\begin{array}{l}0.0013 \\
(17)\end{array}$ & $\begin{array}{l}-0.0148 \\
(16)\end{array}$ \\
\hline O4 & $0.049(2)$ & $0.043(2)$ & $\begin{array}{l}0.0332 \\
(18)\end{array}$ & $\begin{array}{l}0.0186 \\
(19)\end{array}$ & $\begin{array}{l}0.0241 \\
(17)\end{array}$ & $\begin{array}{l}-0.0037 \\
(16)\end{array}$ \\
\hline $\mathrm{C7}$ & $0.021(2)$ & $0.019(2)$ & $\begin{array}{l}0.0194 \\
(19)\end{array}$ & $\begin{array}{l}-0.0005 \\
(17)\end{array}$ & $\begin{array}{l}0.0094 \\
(17)\end{array}$ & $\begin{array}{l}0.0012 \\
(15)\end{array}$ \\
\hline O5 & $0.056(3)$ & $0.036(2)$ & $\begin{array}{l}0.0348 \\
(19)\end{array}$ & $\begin{array}{l}-0.0006 \\
(19)\end{array}$ & $\begin{array}{l}0.0159 \\
(18)\end{array}$ & $\begin{array}{l}0.0141 \\
(16)\end{array}$ \\
\hline 01 & $0.044(2)$ & $0.041(2)$ & $0.061(3)$ & $\begin{array}{l}-0.0100 \\
(19)\end{array}$ & $0.026(2)$ & $\begin{array}{l}0.0172 \\
(19)\end{array}$ \\
\hline O6 & $0.017(2)$ & $0.072(3)$ & $0.043(2)$ & $-0.001(2)$ & $\begin{array}{l}-0.0022 \\
(19)\end{array}$ & 0.007 (2) \\
\hline $\mathrm{C} 4$ & $0.021(2)$ & $0.032(3)$ & $0.027(2)$ & $0.008(2)$ & $\begin{array}{l}0.0091 \\
(19)\end{array}$ & $\begin{array}{l}0.0094 \\
(19)\end{array}$ \\
\hline C1 & $0.017(2)$ & 0.029 (3) & $0.034(2)$ & $\begin{array}{l}-0.0016 \\
(19)\end{array}$ & $0.005(2)$ & $\begin{array}{l}-0.0031 \\
(19)\end{array}$ \\
\hline C5 & $0.024(2)$ & $0.027(2)$ & $0.022(2)$ & $0.005(2)$ & $\begin{array}{l}0.0078 \\
(18)\end{array}$ & $\begin{array}{l}0.0005 \\
(18)\end{array}$ \\
\hline C8 & $0.036(3)$ & $0.026(2)$ & $0.027(2)$ & $0.000(2)$ & $0.014(2)$ & $\begin{array}{l}-0.0064 \\
(18)\end{array}$ \\
\hline $\mathrm{Cg}$ & $0.023(2)$ & $0.019(2)$ & $0.024(2)$ & $\begin{array}{l}0.0017 \\
(18)\end{array}$ & $\begin{array}{l}0.0106 \\
(18)\end{array}$ & $\begin{array}{l}0.0015 \\
(16)\end{array}$ \\
\hline $\mathrm{C} 2$ & $0.025(2)$ & $0.029(2)$ & $0.024(2)$ & $0.003(2)$ & $\begin{array}{l}0.0008 \\
(19)\end{array}$ & $\begin{array}{l}-0.0003 \\
(18)\end{array}$ \\
\hline
\end{tabular}




\begin{tabular}{|l|l|l|l|l|l|l|}
\hline C3 & $0.023(3)$ & $0.026(2)$ & $0.030(2)$ & $\begin{array}{l}-0.0003 \\
(19)\end{array}$ & $0.009(2)$ & $\begin{array}{l}-0.0031 \\
(18)\end{array}$ \\
\hline C6 & $0.025(3)$ & $0.031(2)$ & $0.025(2)$ & $0.004(2)$ & $0.012(2)$ & $\begin{array}{l}0.0021 \\
(19)\end{array}$ \\
\hline C10 & $0.040(3)$ & $0.030(3)$ & $0.038(3)$ & $-0.002(2)$ & $0.014(2)$ & $0.010(2)$ \\
\hline
\end{tabular}

Geometric parameters ( $(\AA, \stackrel{\circ}{)}$ for (2)

\begin{tabular}{|c|c|c|c|}
\hline Os1-Os2 & 2.7419 (3) & $\mathrm{O} 3-\mathrm{C} 3$ & $1.139(6)$ \\
\hline Os1-O9 & $2.113(3)$ & $\mathrm{O} 2-\mathrm{C} 2$ & $1.135(6)$ \\
\hline Os1-O7 & $2.118(3)$ & $\mathrm{O} 4-\mathrm{C} 4$ & $1.137(6)$ \\
\hline Os1-C1 & $1.876(5)$ & C7-C8 & $1.486(6)$ \\
\hline Os1-C2 & $1.881(5)$ & O5-C5 & $1.128(6)$ \\
\hline Os1-C3 & $1.963(5)$ & $\mathrm{O} 1-\mathrm{C} 1$ & $1.131(6)$ \\
\hline Os2-08 & $2.116(3)$ & O6-C6 & $1.124(7)$ \\
\hline Os2-O10 & 2.114 (3) & $\mathrm{C} 8-\mathrm{H} 8 \mathrm{~A}$ & 0.9800 \\
\hline Os2-C4 & $1.877(5)$ & C8-H8B & 0.9800 \\
\hline Os2-C5 & $1.889(5)$ & $\mathrm{C} 8-\mathrm{H} 8 \mathrm{C}$ & 0.9800 \\
\hline Os2-C6 & $1.976(5)$ & $\mathrm{C} 9-\mathrm{C} 10$ & $1.494(6)$ \\
\hline $\mathrm{O} 8-\mathrm{C} 7$ & $1.262(5)$ & $\mathrm{C} 10-\mathrm{H} 10 \mathrm{~A}$ & 0.9800 \\
\hline O9-C9 & $1.268(5)$ & $\mathrm{C} 10-\mathrm{H} 10 \mathrm{~B}$ & 0.9800 \\
\hline $\mathrm{O} 7-\mathrm{C} 7$ & $1.269(5)$ & $\mathrm{C} 10-\mathrm{H} 10 \mathrm{C}$ & 0.9800 \\
\hline O10-C9 & $1.251(5)$ & & \\
\hline O9-Os1-Os2 & $83.54(8)$ & C6-Os2-O10 & $87.60(17)$ \\
\hline O9-Os1-O7 & $83.14(13)$ & $\mathrm{C} 7-\mathrm{O} 8-\mathrm{Os} 2$ & $123.7(3)$ \\
\hline O7-Os1-Os2 & $82.46(8)$ & C9-O9-Os 1 & $122.9(3)$ \\
\hline
\end{tabular}




\begin{tabular}{|l|l|l|l|}
\hline C1-Os1-Os2 & $92.46(14)$ & C7-O7-Os1 & $124.4(3)$ \\
\hline C1-Os1-O9 & $174.06(16)$ & C9-O10-Os2 & $125.3(3)$ \\
\hline C1-Os1-O7 & $92.00(17)$ & O8-C7-O7 & $124.4(4)$ \\
\hline C1-Os1-C2 & $89.8(2)$ & O8-C7-C8 & $118.6(4)$ \\
\hline C1-Os1-C3 & $95.3(2)$ & O7-C7-C8 & $117.0(4)$ \\
\hline C2-Os1-Os2 & $95.05(14)$ & O4-C4-Os2 & $178.3(4)$ \\
\hline C2-Os1-O9 & $94.95(18)$ & O1-C1-Os1 & $177.4(4)$ \\
\hline C2-Os1-O7 & $177.01(18)$ & O5-C5-Os2 & $179.3(5)$ \\
\hline C2-Os1-C3 & $95.8(2)$ & C7-C8-H8A & 109.5 \\
\hline C3-Os1-Os2 & $166.67(14)$ & C7-C8-H8B & 109.5 \\
\hline C3-Os1-O9 & $87.81(17)$ & C7-C8-H8C & 109.5 \\
\hline C3-Os1-O7 & $86.47(17)$ & H8A-C8-H8B & 109.5 \\
\hline O8-Os2-Os1 & $83.26(8)$ & H8A-C8-H8C & 109.5 \\
\hline O10-Os2-Os1 & $82.12(8)$ & H8B-C8-H8C & 109.5 \\
\hline O10-Os2-O8 & $84.12(13)$ & O9-C9-C10 & $117.8(4)$ \\
\hline C4-Os2-Os1 & $92.53(14)$ & O10-C9-O9 & $124.8(4)$ \\
\hline C4-Os2-O8 & $175.04(16)$ & O10-C9-C10 & $117.4(4)$ \\
\hline C4-Os2-O10 & $92.72(16)$ & O2-C2-Os1 & $178.7(4)$ \\
\hline C4-Os2-C5 & $90.8(2)$ & O3-C3-Os1 & $178.3(5)$ \\
\hline C4-Os2-C6 & $94.9(2)$ & O6-C6-Os2 & $178.2(5)$ \\
\hline C5-Os2-Os1 & $95.71(14)$ & C9-C10-H10A & 109.5 \\
\hline C5-Os2-O8 & $92.21(16)$ & C9-C10-H10B & 109.5 \\
\hline C5-Os2-O10 & $175.92(16)$ & C9-C10-H10C & 109.5 \\
\hline C5-Os2-C6 & $94.1(2)$ & H10A-C10- & 109.5 \\
\hline C6-Os2-Os1 & $167.58(15)$ & H10A-C10- & 109.5 \\
\hline
\end{tabular}




\begin{tabular}{|l|l|l|l|}
\hline & & H10C & \\
\hline C6-Os2-O8 & $88.79(17)$ & $\begin{array}{l}\text { H10B-C10- } \\
\text { H10C }\end{array}$ & 109.5 \\
\hline
\end{tabular}

(3)

Crystal data

\begin{tabular}{|l|l|}
\hline $\mathrm{C}_{30} \mathrm{H}_{27} \mathrm{O}_{9} \mathrm{Oss}_{2} \mathrm{P}$ & $Z=2$ \\
\hline$M_{r}=942.88$ & $F(000)=892$ \\
\hline Triclinic, $P \overline{1}$ & $D_{\times}=2.062 \mathrm{Mg} \mathrm{m}^{-3}$ \\
\hline$a=10.452(4) \AA$ & Mo Ka radiation, $\lambda=0.71073 \AA$ \\
\hline$b=11.229(4) \AA$ & Cell parameters from 4436 reflections \\
\hline$c=13.045(5) \AA$ & $\theta=1.8-27.5^{\circ}$ \\
\hline$\alpha=82.813(6)^{\circ}$ & $\mu=8.46 \mathrm{~mm}^{-1}$ \\
\hline$\beta=89.157(8)^{\circ}$ & $T=100 \mathrm{~K}^{\circ}$ \\
\hline$\gamma=89.457(9)^{\circ}$ & Prism, colorless \\
\hline$V=1518.8(10) \AA^{3}$ & $0.27 \times 0.14 \times 0.12 \mathrm{~mm}$ \\
\hline
\end{tabular}

Data collection

\begin{tabular}{|l|l|}
\hline $\begin{array}{l}\text { Rigaku SCX-Mini Mercury 2+ CCD } \\
\text { diffractometer }\end{array}$ & 6534 reflections with $I>2 \sigma(I)$ \\
\hline Radiation source: sealed tube & $R_{\text {int }}=0.032$ \\
\hline $\begin{array}{l}\omega-\text { scans } \\
\begin{array}{l}\text { Absorption correction: multi-scan } \\
A B S C O R \text { (Higashi, 2001) }\end{array}\end{array}$ & $\theta_{\max }=27.4^{\circ}, \theta_{\min }=3.0^{\circ}$ \\
\hline$T_{\min }=0.605, T_{\max }=1.00$ & $h=-13 \rightarrow 13$ \\
\hline 32350 measured reflections & $k=-14 \rightarrow 14$ \\
\hline
\end{tabular}


6915 independent reflections

\section{Refinement}

\begin{tabular}{|c|c|}
\hline Refinement on $F^{2}$ & $\begin{array}{l}\text { Hydrogen site location: inferred from } \\
\text { neighbouring sites }\end{array}$ \\
\hline Least-squares matrix: full & $\mathrm{H}$-atom parameters constrained \\
\hline$R\left[F^{2}>2 \sigma\left(F^{2}\right)\right]=0.020$ & $\begin{array}{l}w=1 /\left[\sigma^{2}\left(F_{\mathrm{o}}^{2}\right)+(0.013 P)^{2}+4.878 P\right] \\
\text { where } P=\left(F_{\mathrm{o}}^{2}+2 F_{\mathrm{c}}^{2}\right) / 3\end{array}$ \\
\hline$w R\left(F^{2}\right)=0.045$ & $(\Delta / \sigma)_{\max }=0.003$ \\
\hline$S=1.02$ & 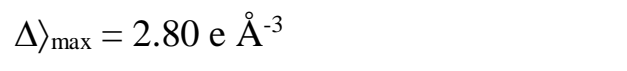 \\
\hline 6915 reflections & $\Delta\rangle_{\min }=-1.05 \mathrm{e} \AA^{-3}$ \\
\hline 385 parameters & $\begin{array}{l}\text { Extinction correction: SHELXL, } \\
\mathrm{Fc}^{*}=\mathrm{kFc}\left[1+0.001 \mathrm{xFc}^{2} \lambda^{3} / \sin (2 \theta)\right]^{-1 / 4}\end{array}$ \\
\hline 0 restraints & Extinction coefficient: 0.00017 (6) \\
\hline
\end{tabular}

Special details

Geometry. All esds (except the esd in the dihedral angle between two I.s. planes) are estimated using the full covariance matrix. The cell esds are taken into account individually in the estimation of esds in distances, angles and torsion angles; correlations between esds in cell parameters are only used when they are defined by crystal symmetry. An approximate (isotropic) treatment of cell esds is used for estimating esds involving I.s. planes.

Fractional atomic coordinates and isotropic or equivalent isotropic displacement parameters $\left(\AA^{2}\right)$ for (3)

\begin{tabular}{|l|l|l|l|l|}
\hline & $x$ & $y$ & $z$ & $U_{\text {iso }} / U_{\text {eq }}$ \\
\hline C1 & $0.1347(3)$ & $0.4868(3)$ & $0.1894(2)$ & $0.0127(6)$ \\
\hline C2 & $0.0634(3)$ & $0.4462(3)$ & $0.2789(2)$ & $0.0145(6)$ \\
\hline
\end{tabular}




\begin{tabular}{|c|c|c|c|c|}
\hline $\mathrm{H} 2$ & 0.0367 & 0.5015 & 0.3245 & $0.017^{\star}$ \\
\hline C3 & $0.0318(3)$ & $0.3263(3)$ & $0.3013(2)$ & $0.0157(6)$ \\
\hline H3 & -0.0176 & 0.3007 & 0.3616 & $0.019^{\star}$ \\
\hline C4 & $0.0714(3)$ & $0.2420(3)$ & 0.2368 (3) & $0.0151(6)$ \\
\hline C5 & $0.1440(3)$ & $0.2823(3)$ & $0.1486(3)$ & $0.0168(6)$ \\
\hline H5 & 0.1730 & 0.2264 & 0.1042 & $0.020^{*}$ \\
\hline C6 & 0.1744 (3) & $0.4033(3)$ & $0.1250(2)$ & $0.0143(6)$ \\
\hline $\mathrm{H} 6$ & 0.2229 & 0.4290 & 0.0642 & $0.017^{*}$ \\
\hline C7 & $0.0330(4)$ & $0.1124(3)$ & $0.2607(3)$ & $0.0238(8)$ \\
\hline H7A & 0.0324 & 0.0893 & 0.3356 & $0.036^{*}$ \\
\hline H7B & 0.0944 & 0.0618 & 0.2281 & $0.036^{*}$ \\
\hline $\mathrm{H} 7 \mathrm{C}$ & -0.0527 & 0.1019 & 0.2338 & $0.036^{\star}$ \\
\hline C8 & 0.2576 (3) & $0.6603(3)$ & $0.0404(2)$ & $0.0125(6)$ \\
\hline C9 & 0.3909 (3) & $0.6544(4)$ & 0.0417 (3) & $0.0263(8)$ \\
\hline H9 & 0.4338 & 0.6500 & 0.1058 & $0.032^{*}$ \\
\hline C10 & $0.4618(3)$ & $0.6549(4)$ & $-0.0489(3)$ & $0.0275(8)$ \\
\hline $\mathrm{H} 10$ & 0.5525 & 0.6521 & -0.0456 & $0.033^{*}$ \\
\hline C11 & 0.4039 (3) & $0.6592(3)$ & $-0.1441(2)$ & $0.0159(6)$ \\
\hline C12 & $0.2701(3)$ & $0.6658(3)$ & $-0.1455(3)$ & $0.0209(7)$ \\
\hline $\mathrm{H} 12$ & 0.2274 & 0.6704 & -0.2097 & $0.025^{*}$ \\
\hline C13 & $0.1983(3)$ & $0.6658(3)$ & $-0.0549(3)$ & $0.0198(7)$ \\
\hline $\mathrm{H} 13$ & 0.1076 & 0.6697 & -0.0581 & $0.024^{*}$ \\
\hline C14 & $0.4829(3)$ & $0.6576(3)$ & $-0.2416(3)$ & $0.0215(7)$ \\
\hline $\mathrm{H} 14 \mathrm{~A}$ & 0.5249 & 0.7353 & -0.2590 & $0.032^{*}$ \\
\hline H14B & 0.4273 & 0.6424 & -0.2983 & $0.032^{*}$ \\
\hline
\end{tabular}




\begin{tabular}{|l|l|l|l|l|}
\hline H14C & 0.5480 & 0.5940 & -0.2308 & $0.032^{\star}$ \\
\hline C15 & $0.0115(3)$ & $0.7157(3)$ & $0.1292(2)$ & $0.0123(6)$ \\
\hline C16 & $0.0042(3)$ & $0.8404(3)$ & $0.1045(3)$ & $0.0173(7)$ \\
\hline H16 & 0.0806 & 0.8861 & 0.0968 & $0.021^{\star}$ \\
\hline C17 & $-0.1134(3)$ & $0.8983(3)$ & $0.0909(3)$ & $0.0186(7)$ \\
\hline H17 & -0.1160 & 0.9830 & 0.0735 & $0.022^{\star}$ \\
\hline C18 & $-0.2277(3)$ & $0.8350(3)$ & $0.1024(2)$ & $0.0180(7)$ \\
\hline C19 & $-0.2203(3)$ & $0.7104(3)$ & $0.1246(3)$ & $0.0182(7)$ \\
\hline H19 & -0.2969 & 0.6650 & 0.1312 & $0.022^{\star}$ \\
\hline C20 & $-0.1024(3)$ & $0.6512(3)$ & $0.1374(3)$ & $0.0168(7)$ \\
\hline H20 & -0.0997 & 0.5661 & 0.1518 & $0.020^{\star}$ \\
\hline C21 & $-0.3553(3)$ & $0.8995(4)$ & $0.0937(3)$ & $0.0271(8)$ \\
\hline H21A & -0.4068 & 0.8674 & 0.0414 & $0.041^{\star}$ \\
\hline H21B & -0.3415 & 0.9855 & 0.0734 & $0.041^{\star}$ \\
\hline H21C & -0.4003 & 0.8874 & 0.1606 & $0.041^{\star}$ \\
\hline C22 & $0.4281(3)$ & $0.6810(3)$ & $0.2888(2)$ & $0.0152(6)$ \\
\hline C23 & $0.3029(3)$ & $0.8760(3)$ & $0.2091(3)$ & $0.0162(6)$ \\
\hline C24 & $0.2352(3)$ & $0.5818(3)$ & $0.5051(3)$ & $0.0148(6)$ \\
\hline C25 & $0.2099(3)$ & $0.4613(3)$ & $0.5673(3)$ & $0.0219(7)$ \\
\hline H25A & 0.1252 & 0.4330 & 0.5505 & $0.033^{\star}$ \\
\hline H25B & 0.2127 & 0.4693 & 0.6412 & $0.033^{\star}$ \\
\hline H25C & 0.2753 & 0.4034 & 0.5505 & $0.033^{\star}$ \\
\hline C26 & $0.0355(3)$ & $0.8338(3)$ & $0.4138(2)$ & $0.0113(6)$ \\
\hline C27 & $-0.1052(3)$ & $0.8548(3)$ & $0.4291(3)$ & $0.0161(6)$ \\
\hline H27A & 0.8404 & 0.3667 & $0.024^{\star}$ \\
\hline & & & & \\
\hline
\end{tabular}




\begin{tabular}{|l|l|l|l|l|}
\hline H27B & -0.1198 & 0.9379 & 0.4423 & $0.024^{*}$ \\
\hline H27C & -0.1368 & 0.7999 & 0.4881 & $0.024^{\star}$ \\
\hline C28 & $0.3296(3)$ & $0.9928(3)$ & $0.4006(3)$ & $0.0169(7)$ \\
\hline C29 & $0.4871(3)$ & $0.8127(3)$ & $0.4656(3)$ & $0.0198(7)$ \\
\hline C30 & $0.3235(3)$ & $0.9004(3)$ & $0.6145(3)$ & $0.0176(7)$ \\
\hline O1 & $0.5308(2)$ & $0.6419(2)$ & $0.28700(19)$ & $0.0244(6)$ \\
\hline O2 & $0.3279(2)$ & $0.9606(2)$ & $0.1523(2)$ & $0.0252(6)$ \\
\hline O3 & $0.2162(2)$ & $0.5898(2)$ & $0.40969(17)$ & $0.0177(5)$ \\
\hline O4 & $0.2747(2)$ & $0.6648(2)$ & $0.55293(18)$ & $0.0173(5)$ \\
\hline O5 & $0.0720(2)$ & $0.7938(2)$ & $0.33261(17)$ & $0.0159(5)$ \\
\hline O6 & $0.1085(2)$ & $0.8585(2)$ & $0.48501(17)$ & $0.0141(4)$ \\
\hline O7 & $0.3398(3)$ & $1.0848(2)$ & $0.3535(2)$ & $0.0280(6)$ \\
\hline O8 & $0.5950(2)$ & $0.7955(3)$ & $0.4569(2)$ & $0.0335(7)$ \\
\hline O9 & $0.3396(3)$ & $0.9413(2)$ & $0.6878(2)$ & $0.0300(6)$ \\
\hline Os1 & $0.26266(2)$ & $0.74048(2)$ & $0.30025(2)$ & $0.01069(4)$ \\
\hline Os2 & $0.31080(2)$ & $0.84058(2)$ & $0.47863(2)$ & $0.01191(4)$ \\
\hline P1 & $0.16761(7)$ & $0.64666(7)$ & $0.16222(6)$ & $0.01095(15)$ \\
\hline
\end{tabular}

Atomic displacement parameters $\left(\AA^{2}\right)$ for $(\mathbf{3})$

\begin{tabular}{|l|l|l|l|l|l|l|}
\hline & $U^{11}$ & $U^{22}$ & $U^{\beta 3}$ & $U^{12}$ & $U^{13}$ & $U^{23}$ \\
\hline C1 & $\begin{array}{l}0.0122 \\
(14)\end{array}$ & $\begin{array}{l}0.0121 \\
(15)\end{array}$ & $\begin{array}{l}0.0139 \\
(15)\end{array}$ & $\begin{array}{l}0.0001 \\
(11)\end{array}$ & $\begin{array}{l}-0.0031 \\
(12)\end{array}$ & -0.0014 \\
& $(12)$ \\
\hline C2 & 0.0146 & 0.0164 & 0.0136 & -0.0008 & 0.0003 & -0.0056 \\
& $(15)$ & $(16)$ & $(15)$ & $(12)$ & $(12)$ & $(12)$ \\
\hline C3 & 0.0140 & 0.0195 & 0.0132 & -0.0031 & -0.0002 & -0.0005 \\
& $(15)$ & $(16)$ & $(15)$ & $(12)$ & $(12)$ & $(12)$ \\
\hline
\end{tabular}




\begin{tabular}{|c|c|c|c|c|c|c|}
\hline C4 & $\begin{array}{l}0.0150 \\
(15)\end{array}$ & $\begin{array}{l}0.0132 \\
(15)\end{array}$ & $\begin{array}{l}0.0176 \\
(16)\end{array}$ & $\begin{array}{l}-0.0012 \\
(12)\end{array}$ & $\begin{array}{l}-0.0043 \\
(12)\end{array}$ & $\begin{array}{l}-0.0026 \\
(12)\end{array}$ \\
\hline C5 & $\begin{array}{l}0.0182 \\
(16)\end{array}$ & $\begin{array}{l}0.0178 \\
(16)\end{array}$ & $\begin{array}{l}0.0158 \\
(16)\end{array}$ & $\begin{array}{l}-0.0001 \\
(13)\end{array}$ & $\begin{array}{l}0.0012 \\
(13)\end{array}$ & $\begin{array}{l}-0.0071 \\
(13)\end{array}$ \\
\hline C6 & $\begin{array}{l}0.0133 \\
(14)\end{array}$ & $\begin{array}{l}0.0185 \\
(16)\end{array}$ & $\begin{array}{l}0.0110 \\
(15)\end{array}$ & $\begin{array}{l}0.0002 \\
(12)\end{array}$ & $\begin{array}{l}0.0014 \\
(12)\end{array}$ & $\begin{array}{l}-0.0019 \\
(12)\end{array}$ \\
\hline C7 & $\begin{array}{l}0.0291 \\
(19)\end{array}$ & $\begin{array}{l}0.0160 \\
(17)\end{array}$ & $\begin{array}{l}0.0263 \\
(19)\end{array}$ & $\begin{array}{l}-0.0043 \\
(14)\end{array}$ & $\begin{array}{l}0.0014 \\
(15)\end{array}$ & $\begin{array}{l}-0.0025 \\
(14)\end{array}$ \\
\hline C8 & $\begin{array}{l}0.0136 \\
(14)\end{array}$ & $\begin{array}{l}0.0121 \\
(14)\end{array}$ & $\begin{array}{l}0.0116 \\
(15)\end{array}$ & $\begin{array}{l}-0.0018 \\
(11)\end{array}$ & $\begin{array}{l}0.0014 \\
(12)\end{array}$ & $\begin{array}{l}-0.0007 \\
(11)\end{array}$ \\
\hline $\mathrm{Cg}$ & $\begin{array}{l}0.0159 \\
(17)\end{array}$ & $0.051(2)$ & $\begin{array}{l}0.0130 \\
(17)\end{array}$ & $\begin{array}{l}0.0006 \\
(16)\end{array}$ & $\begin{array}{l}-0.0027 \\
(13)\end{array}$ & $\begin{array}{l}-0.0080 \\
(16)\end{array}$ \\
\hline $\mathrm{C} 10$ & $\begin{array}{l}0.0116 \\
(16)\end{array}$ & $0.052(3)$ & $\begin{array}{l}0.0196 \\
(18)\end{array}$ & $\begin{array}{l}0.0007 \\
(16)\end{array}$ & $\begin{array}{l}-0.0013 \\
(13)\end{array}$ & $\begin{array}{l}-0.0082 \\
(17)\end{array}$ \\
\hline C11 & $\begin{array}{l}0.0187 \\
(16)\end{array}$ & $\begin{array}{l}0.0151 \\
(15)\end{array}$ & $\begin{array}{l}0.0136 \\
(15)\end{array}$ & $\begin{array}{l}-0.0004 \\
(12)\end{array}$ & $\begin{array}{l}0.0014 \\
(12)\end{array}$ & $\begin{array}{l}-0.0008 \\
(12)\end{array}$ \\
\hline C12 & $\begin{array}{l}0.0182 \\
(16)\end{array}$ & $0.033(2)$ & $\begin{array}{l}0.0107 \\
(16)\end{array}$ & $\begin{array}{l}-0.0011 \\
(14)\end{array}$ & $\begin{array}{l}-0.0025 \\
(13)\end{array}$ & $\begin{array}{l}0.0016 \\
(14)\end{array}$ \\
\hline $\mathrm{C} 13$ & $\begin{array}{l}0.0139 \\
(15)\end{array}$ & $\begin{array}{l}0.0313 \\
(19)\end{array}$ & $\begin{array}{l}0.0137 \\
(16)\end{array}$ & $\begin{array}{l}-0.0017 \\
(14)\end{array}$ & $\begin{array}{l}-0.0017 \\
(12)\end{array}$ & $\begin{array}{l}-0.0003 \\
(14)\end{array}$ \\
\hline C14 & $\begin{array}{l}0.0198 \\
(17)\end{array}$ & $\begin{array}{l}0.0288 \\
(19)\end{array}$ & $\begin{array}{l}0.0150 \\
(16)\end{array}$ & $\begin{array}{l}-0.0015 \\
(14)\end{array}$ & $\begin{array}{l}0.0024 \\
(13)\end{array}$ & $\begin{array}{l}0.0001 \\
(14)\end{array}$ \\
\hline C15 & $\begin{array}{l}0.0113 \\
(14)\end{array}$ & $\begin{array}{l}0.0171 \\
(15)\end{array}$ & $\begin{array}{l}0.0091 \\
(14)\end{array}$ & $\begin{array}{l}-0.0002 \\
(12)\end{array}$ & $\begin{array}{l}-0.0003 \\
(11)\end{array}$ & $\begin{array}{l}-0.0033 \\
(12)\end{array}$ \\
\hline C16 & $\begin{array}{l}0.0161 \\
(15)\end{array}$ & $\begin{array}{l}0.0174 \\
(16)\end{array}$ & $\begin{array}{l}0.0183 \\
(16)\end{array}$ & $\begin{array}{l}-0.0029 \\
(12)\end{array}$ & $\begin{array}{l}-0.0039 \\
(13)\end{array}$ & $\begin{array}{l}-0.0011 \\
(13)\end{array}$ \\
\hline C17 & $\begin{array}{l}0.0229 \\
(17)\end{array}$ & $\begin{array}{l}0.0164 \\
(16)\end{array}$ & $\begin{array}{l}0.0170 \\
(16)\end{array}$ & $\begin{array}{l}0.0029 \\
(13)\end{array}$ & $\begin{array}{l}-0.0032 \\
(13)\end{array}$ & $\begin{array}{l}-0.0033 \\
(13)\end{array}$ \\
\hline C18 & 0.0151 & 0.0289 & 0.0105 & 0.0042 & -0.0001 & -0.0045 \\
\hline
\end{tabular}




\begin{tabular}{|c|c|c|c|c|c|c|}
\hline & (15) & (18) & (15) & (13) & (12) & (13) \\
\hline C19 & $\begin{array}{l}0.0134 \\
(15)\end{array}$ & $\begin{array}{l}0.0254 \\
(18)\end{array}$ & $\begin{array}{l}0.0158 \\
(16)\end{array}$ & $\begin{array}{l}-0.0011 \\
(13)\end{array}$ & $\begin{array}{l}0.0012 \\
(12)\end{array}$ & $\begin{array}{l}-0.0026 \\
(13)\end{array}$ \\
\hline C20 & $\begin{array}{l}0.0161 \\
(15)\end{array}$ & $\begin{array}{l}0.0191 \\
(16)\end{array}$ & $\begin{array}{l}0.0153 \\
(16)\end{array}$ & $\begin{array}{l}-0.0019 \\
(13)\end{array}$ & $\begin{array}{l}0.0003 \\
(12)\end{array}$ & $\begin{array}{l}-0.0020 \\
(13)\end{array}$ \\
\hline C21 & $\begin{array}{l}0.0188 \\
(17)\end{array}$ & $0.035(2)$ & $0.027(2)$ & $\begin{array}{l}0.0091 \\
(15)\end{array}$ & $\begin{array}{l}-0.0022 \\
(15)\end{array}$ & $\begin{array}{l}-0.0020 \\
(16)\end{array}$ \\
\hline C22 & $\begin{array}{l}0.0166 \\
(16)\end{array}$ & $\begin{array}{l}0.0174 \\
(16)\end{array}$ & $\begin{array}{l}0.0123 \\
(15)\end{array}$ & $\begin{array}{l}-0.0021 \\
(12)\end{array}$ & $\begin{array}{l}-0.0022 \\
(12)\end{array}$ & $\begin{array}{l}-0.0043 \\
(12)\end{array}$ \\
\hline C23 & $\begin{array}{l}0.0130 \\
(15)\end{array}$ & $\begin{array}{l}0.0172 \\
(16)\end{array}$ & $\begin{array}{l}0.0191 \\
(17)\end{array}$ & $\begin{array}{l}-0.0014 \\
(12)\end{array}$ & $\begin{array}{l}-0.0008 \\
(12)\end{array}$ & $\begin{array}{l}-0.0049 \\
(13)\end{array}$ \\
\hline C24 & $\begin{array}{l}0.0105 \\
(14)\end{array}$ & $\begin{array}{l}0.0169 \\
(16)\end{array}$ & $\begin{array}{l}0.0169 \\
(16)\end{array}$ & $\begin{array}{l}0.0047 \\
(12)\end{array}$ & $\begin{array}{l}-0.0002 \\
(12)\end{array}$ & $\begin{array}{l}-0.0018 \\
(12)\end{array}$ \\
\hline C25 & $\begin{array}{l}0.0248 \\
(18)\end{array}$ & $\begin{array}{l}0.0175 \\
(17)\end{array}$ & $\begin{array}{l}0.0226 \\
(18)\end{array}$ & $\begin{array}{l}-0.0001 \\
(14)\end{array}$ & $\begin{array}{l}0.0013 \\
(14)\end{array}$ & $\begin{array}{l}0.0010 \\
(14)\end{array}$ \\
\hline C26 & $\begin{array}{l}0.0126 \\
(14)\end{array}$ & $\begin{array}{l}0.0067 \\
(13)\end{array}$ & $\begin{array}{l}0.0139 \\
(15)\end{array}$ & $\begin{array}{l}-0.0001 \\
(11)\end{array}$ & $\begin{array}{l}0.0000 \\
(11)\end{array}$ & $\begin{array}{l}0.0015 \\
(11)\end{array}$ \\
\hline C27 & $\begin{array}{l}0.0107 \\
(14)\end{array}$ & $\begin{array}{l}0.0164 \\
(16)\end{array}$ & $\begin{array}{l}0.0218 \\
(17)\end{array}$ & $\begin{array}{l}0.0017 \\
(12)\end{array}$ & $\begin{array}{l}-0.0004 \\
(12)\end{array}$ & $\begin{array}{l}-0.0046 \\
(13)\end{array}$ \\
\hline C28 & $\begin{array}{l}0.0143 \\
(15)\end{array}$ & $\begin{array}{l}0.0186 \\
(17)\end{array}$ & $\begin{array}{l}0.0184 \\
(16)\end{array}$ & $\begin{array}{l}-0.0022 \\
(12)\end{array}$ & $\begin{array}{l}-0.0020 \\
(12)\end{array}$ & $\begin{array}{l}-0.0046 \\
(13)\end{array}$ \\
\hline C29 & $\begin{array}{l}0.0188 \\
(17)\end{array}$ & $\begin{array}{l}0.0204 \\
(17)\end{array}$ & $\begin{array}{l}0.0231 \\
(18)\end{array}$ & $\begin{array}{l}-0.0023 \\
(13)\end{array}$ & $\begin{array}{l}-0.0044 \\
(14)\end{array}$ & $\begin{array}{l}-0.0137 \\
(14)\end{array}$ \\
\hline C30 & $\begin{array}{l}0.0168 \\
(16)\end{array}$ & $\begin{array}{l}0.0174 \\
(16)\end{array}$ & $\begin{array}{l}0.0194 \\
(17)\end{array}$ & $\begin{array}{l}0.0034 \\
(13)\end{array}$ & $\begin{array}{l}-0.0039 \\
(13)\end{array}$ & $\begin{array}{l}-0.0048 \\
(13)\end{array}$ \\
\hline O1 & $\begin{array}{l}0.0170 \\
(12)\end{array}$ & $\begin{array}{l}0.0357 \\
(15)\end{array}$ & $\begin{array}{l}0.0218 \\
(13)\end{array}$ & $\begin{array}{l}0.0083 \\
(11)\end{array}$ & $\begin{array}{l}-0.0028 \\
(10)\end{array}$ & $\begin{array}{l}-0.0093 \\
(11)\end{array}$ \\
\hline $\mathrm{O} 2$ & $\begin{array}{l}0.0264 \\
(13)\end{array}$ & $\begin{array}{l}0.0222 \\
(13)\end{array}$ & $\begin{array}{l}0.0249 \\
(14)\end{array}$ & $\begin{array}{l}-0.0046 \\
(10)\end{array}$ & $\begin{array}{l}0.0025 \\
(11)\end{array}$ & $\begin{array}{l}0.0052 \\
(11)\end{array}$ \\
\hline
\end{tabular}




\begin{tabular}{|c|c|c|c|c|c|c|}
\hline O3 & $\begin{array}{l}0.0231 \\
(12)\end{array}$ & $\begin{array}{l}0.0169 \\
(12)\end{array}$ & $\begin{array}{l}0.0133 \\
(11)\end{array}$ & $\begin{array}{l}-0.0057 \\
(9)\end{array}$ & $\begin{array}{l}-0.0018 \\
(9)\end{array}$ & $\begin{array}{l}-0.0018 \\
(9)\end{array}$ \\
\hline $\mathrm{O} 4$ & $\begin{array}{l}0.0220 \\
(12)\end{array}$ & $\begin{array}{l}0.0143 \\
(11)\end{array}$ & $\begin{array}{l}0.0154 \\
(12)\end{array}$ & $0.0015(9)$ & $\begin{array}{l}-0.0033 \\
(9)\end{array}$ & $\begin{array}{l}-0.0015 \\
\text { (9) }\end{array}$ \\
\hline O5 & $\begin{array}{l}0.0109 \\
(10)\end{array}$ & $\begin{array}{l}0.0222 \\
(12)\end{array}$ & $\begin{array}{l}0.0156 \\
(11)\end{array}$ & $0.0009(9)$ & $\begin{array}{l}-0.0002 \\
(9)\end{array}$ & $\begin{array}{l}-0.0064 \\
(9)\end{array}$ \\
\hline $\mathrm{O} 6$ & $\begin{array}{l}0.0108 \\
(10)\end{array}$ & $\begin{array}{l}0.0178 \\
(11)\end{array}$ & $\begin{array}{l}0.0147 \\
(11)\end{array}$ & $0.0008(9)$ & $\begin{array}{l}-0.0002 \\
(8)\end{array}$ & $\begin{array}{l}-0.0055 \\
(9)\end{array}$ \\
\hline O7 & $\begin{array}{l}0.0348 \\
(15)\end{array}$ & $\begin{array}{l}0.0190 \\
(13)\end{array}$ & $\begin{array}{l}0.0297 \\
(15)\end{array}$ & $\begin{array}{l}-0.0072 \\
(11)\end{array}$ & $\begin{array}{l}-0.0022 \\
(12)\end{array}$ & $\begin{array}{l}-0.0004 \\
(11)\end{array}$ \\
\hline O8 & $\begin{array}{l}0.0141 \\
(13)\end{array}$ & $\begin{array}{l}0.0394 \\
(16)\end{array}$ & $\begin{array}{l}0.0526 \\
(19)\end{array}$ & $\begin{array}{l}0.0008 \\
(11)\end{array}$ & $\begin{array}{l}-0.0028 \\
(12)\end{array}$ & $\begin{array}{l}-0.0274 \\
(14)\end{array}$ \\
\hline O9 & $\begin{array}{l}0.0373 \\
(15)\end{array}$ & $\begin{array}{l}0.0333 \\
(15)\end{array}$ & $\begin{array}{l}0.0224 \\
(14)\end{array}$ & $\begin{array}{l}0.0082 \\
(12)\end{array}$ & $\begin{array}{l}-0.0084 \\
(12)\end{array}$ & $\begin{array}{l}-0.0151 \\
(12)\end{array}$ \\
\hline Os1 & $\begin{array}{l}0.00957 \\
(6)\end{array}$ & $\begin{array}{l}0.01199 \\
(7)\end{array}$ & $\begin{array}{l}0.01086 \\
(7)\end{array}$ & $\begin{array}{l}-0.00047 \\
(4)\end{array}$ & $\begin{array}{l}0.00070 \\
(4)\end{array}$ & $\begin{array}{l}-0.00293 \\
(4)\end{array}$ \\
\hline Os2 & $\begin{array}{l}0.01044 \\
(6)\end{array}$ & $\begin{array}{l}0.01276 \\
(7)\end{array}$ & $\begin{array}{l}0.01335 \\
(7)\end{array}$ & $\begin{array}{l}-0.00026 \\
(4)\end{array}$ & $\begin{array}{l}-0.00131 \\
(5)\end{array}$ & $\begin{array}{l}-0.00473 \\
(5)\end{array}$ \\
\hline P1 & $0.0100(3)$ & $0.0127(4)$ & $0.0104(4)$ & $\begin{array}{l}-0.0016 \\
(3)\end{array}$ & $0.0017(3)$ & $\begin{array}{l}-0.0024 \\
(3)\end{array}$ \\
\hline
\end{tabular}

Geometric parameters $(\AA \stackrel{\circ}{\circ})$ for (3)

\begin{tabular}{|l|l|l|l|}
\hline $\mathrm{C} 1-\mathrm{C} 6$ & $1.392(4)$ & $\mathrm{C} 18-\mathrm{C} 19$ & $1.395(5)$ \\
\hline $\mathrm{C} 1-\mathrm{C} 2$ & $1.406(4)$ & $\mathrm{C} 18-\mathrm{C} 21$ & $1.511(5)$ \\
\hline $\mathrm{C} 1-\mathrm{P} 1$ & $1.820(3)$ & $\mathrm{C} 19-\mathrm{C} 20$ & $1.397(5)$ \\
\hline $\mathrm{C} 2-\mathrm{C} 3$ & $1.383(4)$ & $\mathrm{C} 19-\mathrm{H} 19$ & 0.9500 \\
\hline $\mathrm{C} 2-\mathrm{H} 2$ & 0.9500 & $\mathrm{C} 20-\mathrm{H} 20$ & 0.9500 \\
\hline $\mathrm{C} 3-\mathrm{C} 4$ & $1.398(5)$ & $\mathrm{C} 21-\mathrm{H} 21 \mathrm{~A}$ & 0.9800 \\
\hline
\end{tabular}




\begin{tabular}{|l|l|l|l|}
\hline C3-H3 & 0.9500 & C21-H21B & 0.9800 \\
\hline C4-C5 & $1.399(5)$ & C21-H21C & 0.9800 \\
\hline C4-C7 & $1.507(5)$ & C22-O1 & $1.157(4)$ \\
\hline C5-C6 & $1.394(5)$ & C22-Os1 & $1.858(3)$ \\
\hline C5-H5 & 0.9500 & C23-O2 & $1.159(4)$ \\
\hline C6-H6 & 0.9500 & C23-Os1 & $1.857(3)$ \\
\hline C7-H7A & 0.9800 & C24-O3 & $1.256(4)$ \\
\hline C7-H7B & 0.9800 & C24-O4 & $1.260(4)$ \\
\hline C7-H7C & 0.9800 & C24-C25 & $1.512(5)$ \\
\hline C8-C13 & $1.391(4)$ & C25-H25A & 0.9800 \\
\hline C8-C9 & $1.394(5)$ & C25-H25B & 0.9800 \\
\hline C8-P1 & $1.825(3)$ & C25-H25C & 0.9800 \\
\hline C9-C10 & $1.386(5)$ & C26-O5 & $1.255(4)$ \\
\hline C9-H9 & 0.9500 & C26-O6 & $1.269(4)$ \\
\hline C10-C11 & $1.385(5)$ & C26-C27 & $1.502(4)$ \\
\hline C10-H10 & 0.9500 & C27-H27A & 0.9800 \\
\hline C11-C12 & $1.400(5)$ & C27-H27B & 0.9800 \\
\hline C11-C14 & $1.508(5)$ & C27-H27C & 0.9800 \\
\hline C12-C13 & $1.391(5)$ & C28-O7 & $1.139(4)$ \\
\hline C12-H12 & 0.9500 & C28-Os2 & $1.887(3)$ \\
\hline C13-H13 & 0.9500 & C29-O8 & $1.148(4)$ \\
\hline C14-H14A & 0.9800 & C29-Os2 & $1.875(3)$ \\
\hline C14-H14B & 0.9800 & C30-O9 & $1.126(4)$ \\
\hline C14-H14C & 0.9800 & C30-Os2 & $1.979(3)$ \\
\hline C15-C20 & $1.395(4)$ & $2.128(2)$ \\
\hline
\end{tabular}




\begin{tabular}{|l|l|l|l|}
\hline C15-C16 & $1.399(4)$ & O4-Os2 & $2.122(2)$ \\
\hline C15-P1 & $1.834(3)$ & O5-Os1 & $2.127(2)$ \\
\hline C16-C17 & $1.388(5)$ & O6-Os2 & $2.124(2)$ \\
\hline C16-H16 & 0.9500 & Os1-P1 & $2.4261(10)$ \\
\hline C17-C18 & $1.392(5)$ & Os1-Os2 & $2.7624(8)$ \\
\hline C17-H17 & 0.9500 & & \\
\hline C6-C1-C2 & $118.4(3)$ & $\begin{array}{l}\text { H21A-C21- } \\
\text { H21B }\end{array}$ & 109.5 \\
\hline C6-C1-P1 & $123.4(2)$ & C18-C21-H21C & 109.5 \\
\hline C2-C1-P1 & $118.3(2)$ & $\begin{array}{l}\text { H21A-C21- } \\
\text { H21C }\end{array}$ & 109.5 \\
\hline C3-C2-C1 & $120.6(3)$ & $\begin{array}{l}\text { H21B-C21- } \\
\text { H21C }\end{array}$ & 109.5 \\
\hline C3-C2-H2 & 119.7 & O1-C22-Os1 & $176.5(3)$ \\
\hline C1-C2-H2 & 119.7 & O2-C23-Os1 & $179.9(4)$ \\
\hline C2-C3-C4 & $121.2(3)$ & O3-C24-O4 & $125.8(3)$ \\
\hline C2-C3-H3 & 119.4 & O3-C24-C25 & $116.7(3)$ \\
\hline C4-C3-H3 & 119.4 & O4-C24-C25 & $117.5(3)$ \\
\hline C3-C4-C5 & $118.1(3)$ & C24-C25-H25A & 109.5 \\
\hline C3-C4-C7 & $120.5(3)$ & C24-C25-H25B & 109.5 \\
\hline C5-C4-C7 & $121.3(3)$ & $\begin{array}{l}\text { H25A-C25- } \\
\text { H25B }\end{array}$ & 109.5 \\
\hline C6-C5-C4 & $120.8(3)$ & C24-C25-H25C & 109.5 \\
\hline C6-C5-H5 & 119.6 & 119.6 & 109.5 \\
\hline C4-C5-H5 & & 109.5 \\
\hline
\end{tabular}




\begin{tabular}{|c|c|c|c|}
\hline & & $\mathrm{H} 25 \mathrm{C}$ & \\
\hline $\mathrm{C} 1-\mathrm{C} 6-\mathrm{C} 5$ & $120.8(3)$ & O5-C26-O6 & 125.1 (3) \\
\hline $\mathrm{C} 1-\mathrm{C} 6-\mathrm{H} 6$ & 119.6 & O5-C26-C27 & $118.1(3)$ \\
\hline $\mathrm{C} 5-\mathrm{C} 6-\mathrm{H} 6$ & 119.6 & $\mathrm{O} 6-\mathrm{C} 26-\mathrm{C} 27$ & $116.7(3)$ \\
\hline $\mathrm{C} 4-\mathrm{C} 7-\mathrm{H} 7 \mathrm{~A}$ & 109.5 & $\mathrm{C} 26-\mathrm{C} 27-\mathrm{H} 27 \mathrm{~A}$ & 109.5 \\
\hline $\mathrm{C} 4-\mathrm{C} 7-\mathrm{H} 7 \mathrm{~B}$ & 109.5 & C26-C27-H27B & 109.5 \\
\hline $\mathrm{H} 7 \mathrm{~A}-\mathrm{C} 7-\mathrm{H} 7 \mathrm{~B}$ & 109.5 & $\begin{array}{l}\mathrm{H} 27 \mathrm{~A}-\mathrm{C} 27- \\
\mathrm{H} 27 \mathrm{~B}\end{array}$ & 109.5 \\
\hline $\mathrm{C} 4-\mathrm{C} 7-\mathrm{H} 7 \mathrm{C}$ & 109.5 & $\mathrm{C} 26-\mathrm{C} 27-\mathrm{H} 27 \mathrm{C}$ & 109.5 \\
\hline $\mathrm{H} 7 \mathrm{~A}-\mathrm{C} 7-\mathrm{H} 7 \mathrm{C}$ & 109.5 & $\begin{array}{l}\text { H27A-C27- } \\
\text { H27C }\end{array}$ & 109.5 \\
\hline $\mathrm{H} 7 \mathrm{~B}-\mathrm{C} 7-\mathrm{H} 7 \mathrm{C}$ & 109.5 & $\begin{array}{l}\text { H27B-C27- } \\
\text { H27C }\end{array}$ & 109.5 \\
\hline $\mathrm{C} 13-\mathrm{C} 8-\mathrm{C} 9$ & $117.8(3)$ & O7-C28-Os2 & $179.4(3)$ \\
\hline $\mathrm{C} 13-\mathrm{C} 8-\mathrm{P} 1$ & $122.5(2)$ & O8-C29-Os2 & 179.5 (3) \\
\hline $\mathrm{C} 9-\mathrm{C} 8-\mathrm{P} 1$ & $119.5(2)$ & O9-C30-Os2 & $173.5(3)$ \\
\hline $\mathrm{C} 10-\mathrm{C} 9-\mathrm{C} 8$ & $121.0(3)$ & $\mathrm{C} 24-\mathrm{O} 3-\mathrm{Os} 1$ & $125.3(2)$ \\
\hline $\mathrm{C} 10-\mathrm{C} 9-\mathrm{H} 9$ & 119.5 & $\mathrm{C} 24-\mathrm{O} 4-\mathrm{Os} 2$ & $122.6(2)$ \\
\hline С8-C9-H9 & 119.5 & C26-O5-Os1 & $125.1(2)$ \\
\hline C11-C10-C9 & $121.8(3)$ & C26-O6-Os2 & $123.45(19)$ \\
\hline $\mathrm{C} 11-\mathrm{C} 10-\mathrm{H} 10$ & 119.1 & C23-Os1-C22 & $90.83(14)$ \\
\hline $\mathrm{C} 9-\mathrm{C} 10-\mathrm{H} 10$ & 119.1 & C23-Os1-O5 & $96.20(12)$ \\
\hline $\mathrm{C} 10-\mathrm{C} 11-\mathrm{C} 12$ & $117.2(3)$ & C22-Os1-O5 & $172.36(12)$ \\
\hline $\mathrm{C} 10-\mathrm{C} 11-\mathrm{C} 14$ & $120.8(3)$ & $\mathrm{C} 23-\mathrm{O} 1-\mathrm{O} 3$ & $177.66(12)$ \\
\hline $\mathrm{C} 12-\mathrm{C} 11-\mathrm{C} 14$ & $122.0(3)$ & $\mathrm{C} 22-\mathrm{Os} 1-\mathrm{O} 3$ & $89.97(12)$ \\
\hline $\mathrm{C} 13-\mathrm{C} 12-\mathrm{C} 11$ & $121.4(3)$ & O5-Os1-O3 & $82.89(9)$ \\
\hline
\end{tabular}




\begin{tabular}{|l|l|l|l|}
\hline C13-C12-H12 & 119.3 & C23-Os1-P1 & $90.97(11)$ \\
\hline C11-C12-H12 & 119.3 & C22-Os1-P1 & $98.03(10)$ \\
\hline C12-C13-C8 & $120.9(3)$ & O5-Os1-P1 & $84.88(7)$ \\
\hline C12-C13-H13 & 119.6 & O3-Os1-P1 & $91.10(7)$ \\
\hline C8-C13-H13 & 119.6 & C23-Os1-Os2 & $96.20(10)$ \\
\hline C11-C14-H14A & 109.5 & C22-Os1-Os2 & $94.14(10)$ \\
\hline C11-C14-H14B & 109.5 & O5-Os1-Os2 & $82.15(6)$ \\
\hline H14A-C14-H14B & 109.5 & O3-Os1-Os2 & $81.55(7)$ \\
\hline C11-C14-H14C & 109.5 & P1-Os1-Os2 & $165.78(2)$ \\
\hline H14A-C14-H14C & 109.5 & C29-Os2-C28 & $89.92(15)$ \\
\hline H14B-C14-H14C & 109.5 & C29-Os2-C30 & $94.50(14)$ \\
\hline C20-C15-C16 & $118.1(3)$ & C28-Os2-C30 & $95.08(14)$ \\
\hline C20-C15-P1 & $123.0(2)$ & C29-Os2-O4 & $93.53(13)$ \\
\hline C16-C15-P1 & $118.6(2)$ & C28-Os2-O4 & $173.31(11)$ \\
\hline C17-C16-C15 & $120.7(3)$ & C30-Os2-O4 & $90.37(12)$ \\
\hline C17-C16-H16 & 119.6 & C29-Os2-O6 & $174.59(11)$ \\
\hline C15-C16-H16 & 119.6 & C28-Os2-O6 & $92.36(12)$ \\
\hline C16-C17-C18 & $121.5(3)$ & C30-Os2-O6 & $90.19(11)$ \\
\hline C16-C17-H17 & 119.3 & O4-Os2-O6 & $83.72(9)$ \\
\hline C18-C17-H17 & 119.3 & C29-Os2-Os1 & $91.62(10)$ \\
\hline C17-C18-C19 & $117.8(3)$ & C28-Os2-Os1 & $90.16(10)$ \\
\hline C17-C18-C21 & $121.0(3)$ & C30-Os2-Os1 & $171.95(10)$ \\
\hline C19-C18-C21 & $121.2(3)$ & O4-Os2-Os1 & $84.02(7)$ \\
\hline C18-C19-C20 & $121.2(3)$ & O6-Os2-Os1 & $83.47(6)$ \\
\hline C18-C19-H19 & 119.4 & C1-C8 & $103.95(14)$ \\
\hline
\end{tabular}




\begin{tabular}{|c|c|c|c|}
\hline $\mathrm{C} 20-\mathrm{C} 19-\mathrm{H} 19$ & 119.4 & $\mathrm{C} 1-\mathrm{P} 1-\mathrm{C} 15$ & $104.43(14)$ \\
\hline $\mathrm{C} 15-\mathrm{C} 20-\mathrm{C} 19$ & $120.6(3)$ & $\mathrm{C} 8-\mathrm{P} 1-\mathrm{C} 15$ & $104.85(14)$ \\
\hline $\mathrm{C} 15-\mathrm{C} 20-\mathrm{H} 20$ & 119.7 & $\mathrm{C} 1-\mathrm{P} 1-\mathrm{Os} 1$ & $116.59(11)$ \\
\hline $\mathrm{C} 19-\mathrm{C} 20-\mathrm{H} 20$ & 119.7 & $\mathrm{C} 8-\mathrm{P} 1-\mathrm{Os} 1$ & $115.81(11)$ \\
\hline $\mathrm{C} 18-\mathrm{C} 21-\mathrm{H} 21 \mathrm{~A}$ & 109.5 & C15-P1-Os1 & $109.99(10)$ \\
\hline $\mathrm{C} 18-\mathrm{C} 21-\mathrm{H} 21 \mathrm{~B}$ & 109.5 & & \\
\hline $\mathrm{C} 6-\mathrm{C} 1-\mathrm{C} 2-\mathrm{C} 3$ & $-1.1(5)$ & $\begin{array}{l}\mathrm{P} 1-\mathrm{C} 15-\mathrm{C} 20- \\
\mathrm{C} 19\end{array}$ & $171.4(2)$ \\
\hline $\mathrm{P} 1-\mathrm{C} 1-\mathrm{C} 2-\mathrm{C} 3$ & $177.7(2)$ & $\begin{array}{l}\mathrm{C} 18-\mathrm{C} 19-\mathrm{C} 20- \\
\mathrm{C} 15\end{array}$ & $0.6(5)$ \\
\hline $\mathrm{C} 1-\mathrm{C} 2-\mathrm{C} 3-\mathrm{C} 4$ & $1.0(5)$ & $\begin{array}{l}\mathrm{O} 4-\mathrm{C} 24-\mathrm{O} 3- \\
\text { Os1 }\end{array}$ & $6.4(5)$ \\
\hline $\mathrm{C} 2-\mathrm{C} 3-\mathrm{C} 4-\mathrm{C} 5$ & $0.0(5)$ & $\begin{array}{l}\text { C25-C24-O3- } \\
\text { Os1 }\end{array}$ & $-172.8(2)$ \\
\hline $\mathrm{C} 2-\mathrm{C} 3-\mathrm{C} 4-\mathrm{C} 7$ & $-178.3(3)$ & $\begin{array}{l}\mathrm{O} 3-\mathrm{C} 24-\mathrm{O} 4- \\
\text { Os2 }\end{array}$ & $2.3(4)$ \\
\hline $\mathrm{C} 3-\mathrm{C} 4-\mathrm{C} 5-\mathrm{C} 6$ & $-0.9(5)$ & $\begin{array}{l}\text { C25-C24-O4- } \\
\text { Os2 }\end{array}$ & $-178.5(2)$ \\
\hline $\mathrm{C} 7-\mathrm{C} 4-\mathrm{C} 5-\mathrm{C} 6$ & $177.3(3)$ & $\begin{array}{l}\mathrm{O} 6-\mathrm{C} 26-\mathrm{O} 5- \\
\text { Os1 }\end{array}$ & $6.5(4)$ \\
\hline $\mathrm{C} 2-\mathrm{C} 1-\mathrm{C} 6-\mathrm{C} 5$ & $0.2(5)$ & $\begin{array}{l}\text { C27-C26-O5- } \\
\text { Os1 }\end{array}$ & $-173.6(2)$ \\
\hline $\mathrm{P} 1-\mathrm{C} 1-\mathrm{C} 6-\mathrm{C} 5$ & $-178.6(2)$ & $\begin{array}{l}\text { O5-C26-O6- } \\
\text { Os2 }\end{array}$ & $1.6(4)$ \\
\hline $\mathrm{C} 4-\mathrm{C} 5-\mathrm{C} 6-\mathrm{C} 1$ & $0.8(5)$ & $\begin{array}{l}\text { C27-C26-O6- } \\
\text { Os2 }\end{array}$ & $-178.3(2)$ \\
\hline $\mathrm{C} 13-\mathrm{C} 8-\mathrm{C} 9-\mathrm{C} 10$ & $0.4(6)$ & $\mathrm{C} 6-\mathrm{C} 1-\mathrm{P} 1-\mathrm{C} 8$ & $-0.2(3)$ \\
\hline
\end{tabular}




\begin{tabular}{|c|c|c|c|}
\hline $\mathrm{P} 1-\mathrm{C} 8-\mathrm{C} 9-\mathrm{C} 10$ & $174.8(3)$ & $\mathrm{C} 2-\mathrm{C} 1-\mathrm{P} 1-\mathrm{C} 8$ & $-178.9(2)$ \\
\hline $\mathrm{C} 8-\mathrm{C} 9-\mathrm{C} 10-\mathrm{C} 11$ & $-1.1(6)$ & $\mathrm{C} 6-\mathrm{C} 1-\mathrm{P} 1-\mathrm{C} 15$ & $109.5(3)$ \\
\hline $\begin{array}{l}\mathrm{C} 9-\mathrm{C} 10-\mathrm{C} 11- \\
\mathrm{C} 12\end{array}$ & $1.4(6)$ & $\mathrm{C} 2-\mathrm{C} 1-\mathrm{P} 1-\mathrm{C} 15$ & $-69.2(3)$ \\
\hline $\begin{array}{l}\mathrm{C} 9-\mathrm{C} 10-\mathrm{C} 11- \\
\mathrm{C} 14\end{array}$ & $-179.0(4)$ & $\mathrm{C} 6-\mathrm{C} 1-\mathrm{P} 1-\mathrm{Os} 1$ & $-128.9(2)$ \\
\hline $\begin{array}{l}\mathrm{C} 10-\mathrm{C} 11-\mathrm{C} 12- \\
\mathrm{C} 13\end{array}$ & $-1.2(5)$ & $\mathrm{C} 2-\mathrm{C} 1-\mathrm{P} 1-\mathrm{Os} 1$ & $52.3(3)$ \\
\hline $\begin{array}{l}\mathrm{C} 14-\mathrm{C} 11-\mathrm{C} 12- \\
\mathrm{C} 13\end{array}$ & $179.3(3)$ & $\mathrm{C} 13-\mathrm{C} 8-\mathrm{P} 1-\mathrm{C} 1$ & $82.1(3)$ \\
\hline $\begin{array}{l}\text { C11-C12-C13- } \\
\text { C8 }\end{array}$ & $0.6(6)$ & $\mathrm{C} 9-\mathrm{C} 8-\mathrm{P} 1-\mathrm{C} 1$ & $-92.0(3)$ \\
\hline $\mathrm{C} 9-\mathrm{C} 8-\mathrm{C} 13-\mathrm{C} 12$ & $-0.2(5)$ & $\begin{array}{l}\mathrm{C} 13-\mathrm{C} 8-\mathrm{P} 1- \\
\mathrm{C} 15\end{array}$ & $-27.3(3)$ \\
\hline $\mathrm{P} 1-\mathrm{C} 8-\mathrm{C} 13-\mathrm{C} 12$ & $-174.4(3)$ & C9-C8-P1-C15 & $158.6(3)$ \\
\hline $\begin{array}{l}\mathrm{C} 20-\mathrm{C} 15-\mathrm{C} 16- \\
\mathrm{C} 17\end{array}$ & $1.5(5)$ & $\begin{array}{l}\text { C13-C8-P1- } \\
\text { Os1 }\end{array}$ & $-148.7(2)$ \\
\hline $\begin{array}{l}\mathrm{P} 1-\mathrm{C} 15-\mathrm{C} 16- \\
\mathrm{C} 17\end{array}$ & $-172.3(3)$ & $\mathrm{C} 9-\mathrm{C} 8-\mathrm{P} 1-\mathrm{Os} 1$ & $37.2(3)$ \\
\hline $\begin{array}{l}\mathrm{C} 15-\mathrm{C} 16-\mathrm{C} 17- \\
\mathrm{C} 18\end{array}$ & $0.7(5)$ & $\begin{array}{l}\mathrm{C} 20-\mathrm{C} 15-\mathrm{P} 1- \\
\mathrm{C} 1\end{array}$ & $6.1(3)$ \\
\hline $\begin{array}{l}\text { C16-C17-C18- } \\
\text { C19 }\end{array}$ & $-2.2(5)$ & $\begin{array}{l}\mathrm{C} 16-\mathrm{C} 15-\mathrm{P} 1- \\
\mathrm{C} 1\end{array}$ & $179.7(3)$ \\
\hline $\begin{array}{l}\mathrm{C} 16-\mathrm{C} 17-\mathrm{C} 18- \\
\mathrm{C} 21\end{array}$ & $176.4(3)$ & $\begin{array}{l}\mathrm{C} 20-\mathrm{C} 15-\mathrm{P} 1- \\
\mathrm{C} 8\end{array}$ & $115.2(3)$ \\
\hline $\begin{array}{l}\text { C17-C18-C19- } \\
\text { C20 }\end{array}$ & $1.5(5)$ & $\begin{array}{l}\mathrm{C} 16-\mathrm{C} 15-\mathrm{P} 1- \\
\mathrm{C} 8\end{array}$ & $-71.3(3)$ \\
\hline $\begin{array}{l}\text { C21-C18-C19- } \\
\text { C20 }\end{array}$ & $-177.0(3)$ & $\begin{array}{l}\text { C20-C15-P1- } \\
\text { Os1 }\end{array}$ & $-119.7(3)$ \\
\hline
\end{tabular}




\begin{tabular}{|l|l|l|l|}
\hline $\begin{array}{l}\text { C16-C15-C20- } \\
\text { C19 }\end{array}$ & $-2.2(5)$ & $\begin{array}{l}\text { C16-C15-P1- } \\
\text { Os1 }\end{array}$ & $53.8(3)$ \\
\hline
\end{tabular}

\section{(4) $\cdot 1.5 \mathrm{C}_{8} \mathrm{H}_{10}$}

Crystal data

\begin{tabular}{|l|l|}
\hline $\mathrm{C}_{50} \mathrm{H}_{48} \mathrm{O}_{8} \mathrm{Os}_{2} \mathrm{P}_{2} \cdot 1.5 \mathrm{C}_{8} \mathrm{H}_{10}$ & $Z=2$ \\
\hline$M_{r}=1378.46$ & $F(000)=1362$ \\
\hline Triclinic, $P \overline{1}$ & $D_{x}=1.658 \mathrm{Mg} \mathrm{m}^{-3}$ \\
\hline$a=12.81225(16) \AA$ & Cu Ka radiation, $\lambda=1.54184 \AA$ \\
\hline$b=14.8455(2) \AA$ & Cell parameters from 23482 reflections \\
\hline$C=16.6818(2) \AA$ & $\theta=3.7-73.2^{\circ}$ \\
\hline$\alpha=98.1785(11)^{\circ}$ & $\mu=9.55 \mathrm{~mm}^{-1}$ \\
\hline$\beta=101.7904(11)^{\circ}$ & $T=293 \mathrm{~K}^{\circ}$ \\
\hline$\gamma=113.3846(14)^{\circ}$ & Rectangular plate, clear pale yellow \\
\hline$V=2761.37(7) \AA^{3}$ & $0.11 \times 0.07 \times 0.02 \mathrm{~mm}$ \\
\hline
\end{tabular}

Data collection

\begin{tabular}{|c|c|}
\hline $\begin{array}{l}\text { Rigaku SuperNova AtlasS2 CCD } \\
\text { diffractometer }\end{array}$ & 9768 independent reflections \\
\hline $\begin{array}{l}\text { Radiation source: micro-focus sealed X-ray } \\
\text { tube, SuperNova }(\mathrm{Cu}) \text { X-ray Source }\end{array}$ & 8691 reflections with $I>2 \sigma(I)$ \\
\hline Mirror monochromator & $R_{\text {int }}=0.066$ \\
\hline Detector resolution: 5.2387 pixels $\mathrm{mm}^{-1}$ & $\theta_{\max }=66.6^{\circ}, \theta_{\min }=3.4^{\circ}$ \\
\hline$\omega$ scans & $h=-15 \rightarrow 15$ \\
\hline $\begin{array}{l}\text { Absorption correction: gaussian CrysAlis } \\
P R O \text { (Rigaku OD, 2017) }\end{array}$ & $k=-17 \rightarrow 17$ \\
\hline
\end{tabular}




\begin{tabular}{|l|l|}
\hline$T_{\min }=0.626, T_{\max }=1.000$ & $I=-19 \rightarrow 19$ \\
\hline 49090 measured reflections & \\
\hline
\end{tabular}

Refinement

\begin{tabular}{|l|l|}
\hline Refinement on $F^{2}$ & 0 restraints \\
\hline Least-squares matrix: full & $\begin{array}{l}\text { Hydrogen site location: inferred from } \\
\text { neighbouring sites }\end{array}$ \\
\hline$R\left[F^{2}>2 \sigma\left(F^{2}\right)\right]=0.027$ & $\mathrm{H}$-atom parameters constrained \\
\hline$w R\left(F^{2}\right)=0.068$ & $\begin{array}{l}W=1 /\left[\sigma^{2}\left(F_{0}^{2}\right)+(0.0326 P)^{2}+0.5818 P\right] \\
\text { where } P=\left(F_{0}^{2}+2 F_{c}^{2}\right) / 3\end{array}$ \\
\hline$S=1.05$ & $(\Delta / \sigma)_{\max }=0.009$ \\
\hline 9768 reflections & $\Delta\rangle_{\max }=2.09 \mathrm{e} \AA^{-3}$ \\
\hline 678 parameters & $\Delta\rangle_{\min }=-1.11 \mathrm{e} \AA^{-3}$ \\
\hline
\end{tabular}

Special details

Geometry. All esds (except the esd in the dihedral angle between two I.s. planes) are estimated using the full covariance matrix. The cell esds are taken into account individually in the estimation of esds in distances, angles and torsion angles; correlations between esds in cell parameters are only used when they are defined by crystal symmetry. An approximate (isotropic) treatment of cell esds is used for estimating esds involving l.s. planes.

Fractional atomic coordinates and isotropic or equivalent isotropic displacement parameters $\left(\AA^{2}\right)$ for (4) $1.5 \mathrm{C}_{8} \mathrm{H}_{10}$

\begin{tabular}{|l|l|l|l|l|}
\hline & $x$ & $y$ & $z$ & $U_{\text {iso }} / U_{\text {eq }}$ \\
\hline Os1 & $0.52561(2)$ & $0.31103(2)$ & $0.17940(2)$ & $0.01350(5)$ \\
\hline Os2 & $0.72811(2)$ & $0.49183(2)$ & $0.22971(2)$ & $0.01374(5)$ \\
\hline P1 & $0.32377(7)$ & $0.18569(6)$ & $0.15247(6)$ & $0.01582(17)$ \\
\hline
\end{tabular}




\begin{tabular}{|c|c|c|c|c|}
\hline P2 & $0.87312(7)$ & $0.66724(6)$ & $0.28705(5)$ & $0.01529(17)$ \\
\hline O1 & $0.6466(2)$ & $0.2025(2)$ & $0.27154(18)$ & $0.0273(6)$ \\
\hline $\mathrm{O} 2$ & $0.5582(2)$ & $0.2355(2)$ & $0.01363(17)$ & $0.0262(6)$ \\
\hline $\mathrm{O} 3$ & $0.8908(2)$ & $0.3974(2)$ & $0.28500(19)$ & $0.0293(6)$ \\
\hline $\mathrm{O} 4$ & $0.7791(2)$ & $0.4713(2)$ & $0.06201(18)$ & $0.0302(6)$ \\
\hline $\mathrm{O} 5$ & $0.4957(2)$ & $0.37716(19)$ & $0.28926(16)$ & $0.0191(5)$ \\
\hline O6 & $0.6814(2)$ & $0.4997(2)$ & $0.34497(16)$ & $0.0229(6)$ \\
\hline $\mathrm{O} 7$ & $0.4501(2)$ & $0.39783(19)$ & $0.11990(16)$ & $0.0201(5)$ \\
\hline O8 & $0.6001(2)$ & $0.54765(18)$ & $0.19350(17)$ & $0.0210(5)$ \\
\hline C1 & $0.5991(3)$ & $0.2435(3)$ & $0.2357(2)$ & $0.0202(8)$ \\
\hline C2 & $0.5478(3)$ & $0.2634(3)$ & $0.0782(2)$ & $0.0191(8)$ \\
\hline C3 & $0.8295(3)$ & $0.4349(3)$ & $0.2647(2)$ & $0.0205(8)$ \\
\hline C4 & $0.7596(3)$ & 0.4799 (3) & $0.1262(2)$ & $0.0204(8)$ \\
\hline C5 & $0.5784(3)$ & $0.4483(3)$ & $0.3484(2)$ & $0.0208(8)$ \\
\hline C6 & $0.5499(4)$ & $0.4710(3)$ & $0.4293(3)$ & $0.0281(9)$ \\
\hline $\mathrm{H} 6 \mathrm{~A}$ & 0.471410 & 0.466933 & 0.416934 & $0.042^{*}$ \\
\hline H6B & 0.606215 & 0.537999 & 0.461884 & $0.042^{*}$ \\
\hline $\mathrm{H} 6 \mathrm{C}$ & 0.553879 & 0.422658 & 0.460874 & $0.042^{*}$ \\
\hline $\mathrm{C} 7$ & $0.4981(3)$ & $0.4930(3)$ & $0.1434(2)$ & $0.0214(8)$ \\
\hline C8 & $0.4266(4)$ & $0.5450(3)$ & $0.1082(3)$ & $0.0332(10)$ \\
\hline H8A & 0.423870 & 0.542089 & 0.049895 & $0.050^{*}$ \\
\hline H8B & 0.462793 & 0.614544 & 0.139633 & $0.050^{*}$ \\
\hline $\mathrm{H} 8 \mathrm{C}$ & 0.347401 & 0.511789 & 0.112562 & $0.050^{*}$ \\
\hline C9 & $0.2214(3)$ & $0.2385(3)$ & $0.1172(2)$ & $0.0184(7)$ \\
\hline C10 & $0.1934(3)$ & 0.2931 (3) & $0.1778(2)$ & $0.0198(7)$ \\
\hline
\end{tabular}




\begin{tabular}{|l|l|l|l|l|}
\hline H10 & 0.216126 & 0.292314 & 0.234216 & $0.024^{\star}$ \\
\hline C11 & $0.1323(3)$ & $0.3483(3)$ & $0.1547(2)$ & $0.0224(8)$ \\
\hline H11 & 0.114982 & 0.384548 & 0.195946 & $0.027^{\star}$ \\
\hline C12 & $0.0959(3)$ & $0.3506(3)$ & $0.0702(3)$ & $0.0229(8)$ \\
\hline C13 & $0.1244(3)$ & $0.2967(3)$ & $0.0105(3)$ & $0.0268(8)$ \\
\hline H13 & 0.101210 & 0.297283 & -0.045899 & $0.032^{\star}$ \\
\hline C14 & $0.1866(3)$ & $0.2418(3)$ & $0.0330(2)$ & $0.0233(8)$ \\
\hline H14 & 0.205414 & 0.206952 & -0.008293 & $0.028^{\star}$ \\
\hline C15 & $0.0313(4)$ & $0.4125(3)$ & $0.0454(3)$ & $0.0313(9)$ \\
\hline H15A & -0.005020 & 0.424502 & 0.088157 & $0.047^{\star}$ \\
\hline H15B & -0.028828 & 0.376251 & -0.007556 & $0.047^{\star}$ \\
\hline H15C & 0.086583 & 0.476050 & 0.039950 & $0.047^{\star}$ \\
\hline C16 & $0.2803(3)$ & $0.0689(3)$ & $0.0732(2)$ & $0.0183(7)$ \\
\hline C17 & $0.1659(3)$ & $0.0121(3)$ & $0.0176(2)$ & $0.0228(8)$ \\
\hline H17 & 0.108135 & 0.034979 & 0.018611 & $0.027^{\star}$ \\
\hline C18 & $0.1379(3)$ & $-0.0777(3)$ & $-0.0388(2)$ & $0.0237(8)$ \\
\hline H18 & 0.061579 & -0.114091 & -0.075470 & $0.028^{\star}$ \\
\hline C19 & $0.2219(3)$ & $-0.1146(3)$ & $-0.0416(2)$ & $0.0197(7)$ \\
\hline C20 & $0.3357(3)$ & $-0.0581(3)$ & $0.0153(2)$ & $0.0208(7)$ \\
\hline H20 & 0.393119 & -0.081477 & 0.015326 & $0.025^{\star}$ \\
\hline C21 & $0.3637(3)$ & $0.0315(3)$ & $0.0712(2)$ & $0.0210(8)$ \\
\hline H21 & 0.439844 & 0.067623 & 0.108184 & $0.025^{\star}$ \\
\hline C22 & $0.1942(3)$ & $-0.2102(3)$ & $-0.1050(2)$ & $0.0250(8)$ \\
\hline H22A & -0.194378 & -0.140910 & $0.037^{\star}$ \\
\hline H22B & -0.239125 & -0.138782 & $0.037^{\star}$ \\
\hline & & & \\
\hline
\end{tabular}




\begin{tabular}{|l|l|l|l|l|}
\hline H22C & 0.206713 & -0.257824 & -0.075699 & $0.037^{\star}$ \\
\hline C23 & $0.2753(3)$ & $0.1389(3)$ & $0.2410(2)$ & $0.0183(7)$ \\
\hline C24 & $0.1564(3)$ & $0.0729(3)$ & $0.2272(3)$ & $0.0235(8)$ \\
\hline H24 & 0.104064 & 0.050648 & 0.173139 & $0.028^{\star}$ \\
\hline C25 & $0.1153(3)$ & $0.0399(3)$ & $0.2936(3)$ & $0.0232(8)$ \\
\hline H25 & 0.035691 & -0.004162 & 0.283412 & $0.028^{\star}$ \\
\hline C26 & $0.1916(3)$ & $0.0719(3)$ & $0.3750(3)$ & $0.0229(8)$ \\
\hline C27 & $0.3100(3)$ & $0.1376(3)$ & $0.3879(3)$ & $0.0264(8)$ \\
\hline H27 & 0.362345 & 0.160071 & 0.442027 & $0.032^{\star}$ \\
\hline C28 & $0.3521(3)$ & $0.1706(3)$ & $0.3214(3)$ & $0.0245(8)$ \\
\hline H28 & 0.431883 & 0.213904 & 0.331386 & $0.029^{\star}$ \\
\hline C29 & $0.1465(4)$ & $0.0355(3)$ & $0.4471(3)$ & $0.0323(9)$ \\
\hline H29A & 0.080771 & 0.050251 & 0.450432 & $0.048^{\star}$ \\
\hline H29B & 0.208936 & 0.069394 & 0.499248 & $0.048^{\star}$ \\
\hline H29C & 0.121051 & -0.036301 & 0.437346 & $0.048^{\star}$ \\
\hline C30 & $0.9286(3)$ & $0.7089(3)$ & $0.4023(2)$ & $0.0178(7)$ \\
\hline C31 & $0.8474(3)$ & $0.7050(3)$ & $0.4485(2)$ & $0.0198(7)$ \\
\hline H31 & 0.767888 & 0.683094 & 0.420013 & $0.024^{\star}$ \\
\hline C32 & $0.8847(3)$ & $0.7333(3)$ & $0.5358(2)$ & $0.0207(7)$ \\
\hline H32 & 1.029639 & 0.730875 & 0.565175 & $0.025^{\star}$ \\
\hline C33 & $1.0028(3)$ & $0.7654(3)$ & $0.5810(2)$ & $0.0218(8)$ \\
\hline C34 & $1.161989(3)$ & $0.7684(3)$ & $0.5350(2)$ & $0.0228(8)$ \\
\hline H34 & 0.789112 & 0.563842 & $0.027^{\star}$ \\
\hline C35 & $0.7413(3)$ & $0.4474(2)$ & $0.0206(7)$ \\
\hline H35 & 0.744667 & 0.418307 & $0.025^{\star}$ \\
\hline & & & & \\
\hline
\end{tabular}




\begin{tabular}{|l|l|l|l|l|}
\hline C36 & $1.0416(4)$ & $0.7940(3)$ & $0.6762(2)$ & $0.0286(9)$ \\
\hline H36A & 1.038792 & 0.856629 & 0.696442 & $0.043^{\star}$ \\
\hline H36B & 1.121316 & 0.801975 & 0.696327 & $0.043^{\star}$ \\
\hline H36C & 0.989527 & 0.741690 & 0.696652 & $0.043^{\star}$ \\
\hline C37 & $1.0046(3)$ & $0.6985(3)$ & $0.2496(2)$ & $0.0173(7)$ \\
\hline C38 & $1.0630(3)$ & $0.6365(3)$ & $0.2544(3)$ & $0.0235(8)$ \\
\hline H38 & 1.038309 & 0.583498 & 0.280537 & $0.028^{\star}$ \\
\hline C39 & $1.1576(3)$ & $0.6532(3)$ & $0.2204(3)$ & $0.0244(8)$ \\
\hline H39 & 1.196303 & 0.612016 & 0.225191 & $0.029^{\star}$ \\
\hline C40 & $1.1954(3)$ & $0.7304(3)$ & $0.1796(3)$ & $0.0258(8)$ \\
\hline C41 & $1.1394(3)$ & $0.7934(3)$ & $0.1769(3)$ & $0.0270(9)$ \\
\hline H41 & 1.164746 & 0.846531 & 0.151005 & $0.032^{\star}$ \\
\hline C42 & $1.0463(3)$ & $0.7788(3)$ & $0.2120(3)$ & $0.0244(8)$ \\
\hline H42 & 1.011337 & 0.822979 & 0.210336 & $0.029^{\star}$ \\
\hline C43 & $1.2929(4)$ & $0.7427(4)$ & $0.1380(3)$ & $0.0337(10)$ \\
\hline H43A & 1.357108 & 0.738327 & 0.175825 & $0.050^{\star}$ \\
\hline H43B & 1.321035 & 0.807465 & 0.124746 & $0.050^{\star}$ \\
\hline H43C & 1.262384 & 0.689978 & 0.086924 & $0.050^{\star}$ \\
\hline C44 & $0.8176(3)$ & $0.7585(3)$ & $0.2589(2)$ & $0.0170(7)$ \\
\hline C45 & $0.8316(3)$ & $0.8426(3)$ & $0.3172(2)$ & $0.0195(7)$ \\
\hline H45 & 0.874379 & 0.856310 & 0.373248 & $0.023^{\star}$ \\
\hline C46 & $0.7829(3)$ & $0.9061(3)$ & $0.2928(3)$ & $0.0237(8)$ \\
\hline H46 & 0.793734 & 0.961822 & 0.332987 & $0.028^{\star}$ \\
\hline C47 $(3)$ & $0.8052(3)$ & $0.1513(3)$ & $0.0248(8)$ \\
\hline C48 & $0.8885(3)$ & $0.2098(3)$ & $0.0240(8)$ \\
\hline & & & \\
\hline
\end{tabular}




\begin{tabular}{|c|c|c|c|c|}
\hline $\mathrm{H} 48$ & 0.664560 & 0.792533 & 0.095008 & $0.030^{*}$ \\
\hline C49 & $0.7538(3)$ & $0.7410(3)$ & $0.1748(2)$ & $0.0211(7)$ \\
\hline $\mathrm{H} 49$ & 0.743559 & 0.685698 & 0.134480 & $0.025^{\star}$ \\
\hline C50 & $0.6617(4)$ & $0.9549(3)$ & $0.1835(3)$ & $0.0347(10)$ \\
\hline $\mathrm{H} 50 \mathrm{~A}$ & 0.576957 & 0.917897 & 0.169453 & $0.052^{*}$ \\
\hline $\mathrm{H} 50 \mathrm{~B}$ & 0.683391 & 0.975972 & 0.135111 & $0.052^{*}$ \\
\hline $\mathrm{H} 50 \mathrm{C}$ & 0.688619 & 1.013497 & 0.229212 & $0.052^{*}$ \\
\hline C51 & $0.4260(4)$ & $0.6645(3)$ & $0.3715(3)$ & $0.0303(9)$ \\
\hline C52 & 0.4041 (3) & $0.7228(3)$ & $0.4328(3)$ & $0.0308(9)$ \\
\hline H52 & 0.453454 & 0.791965 & 0.452394 & $0.037^{*}$ \\
\hline C53 & $0.3088(4)$ & $0.6788(3)$ & 0.4654 (3) & $0.0298(9)$ \\
\hline H53 & 0.296385 & 0.719046 & 0.507002 & $0.036^{*}$ \\
\hline C54 & $0.2323(3)$ & $0.5762(3)$ & $0.4369(3)$ & $0.0269(9)$ \\
\hline C55 & $0.2548(4)$ & $0.5190(3)$ & $0.3750(3)$ & $0.0285(9)$ \\
\hline H55 & 0.204875 & 0.449997 & 0.354793 & $0.034^{*}$ \\
\hline C56 & $0.3493(4)$ & $0.5618(3)$ & 0.3425 (3) & $0.0302(9)$ \\
\hline H56 & 0.361549 & 0.521472 & 0.300929 & $0.036^{*}$ \\
\hline C57 & $0.5316(4)$ & $0.7132(4)$ & $0.3385(3)$ & $0.0383(10)$ \\
\hline $\mathrm{H} 57 \mathrm{~A}$ & 0.546104 & 0.661414 & 0.308249 & $0.057^{*}$ \\
\hline H57B & 0.600382 & 0.756331 & 0.385135 & $0.057^{*}$ \\
\hline $\mathrm{H} 57 \mathrm{C}$ & 0.515246 & 0.752699 & 0.301284 & $0.057^{*}$ \\
\hline C58 & $0.1317(4)$ & $0.5287(3)$ & $0.4742(3)$ & $0.0360(10)$ \\
\hline $\mathrm{H} 58 \mathrm{~A}$ & 0.063317 & 0.478694 & 0.430344 & $0.054^{*}$ \\
\hline H58B & 0.112728 & 0.580063 & 0.499867 & $0.054^{*}$ \\
\hline H58C & 0.155174 & 0.497014 & 0.516227 & $0.054^{*}$ \\
\hline
\end{tabular}




\begin{tabular}{|l|l|l|l|l|}
\hline C59 & $0.6146(4)$ & $0.0436(4)$ & $0.5492(4)$ & $0.0483(14)$ \\
\hline H59 & 0.693393 & 0.073771 & 0.581970 & $0.058^{\star}$ \\
\hline C60 & $0.5899(5)$ & $0.0302(3)$ & $0.4632(4)$ & $0.0484(14)$ \\
\hline H60 & 0.652302 & 0.051126 & 0.439229 & $0.058^{\star}$ \\
\hline C61 & $0.4750(5)$ & $-0.0134(3)$ & $0.4113(4)$ & $0.0434(12)$ \\
\hline C62 & $0.4498(7)$ & $-0.0286(5)$ & $0.3172(4)$ & $0.0687(18)$ \\
\hline H62A & 0.370174 & -0.038161 & 0.293340 & $0.103^{*}$ \\
\hline H62B & 0.458306 & -0.087231 & 0.293593 & $0.103^{*}$ \\
\hline H62C & 0.504674 & 0.029967 & 0.304633 & $0.103^{\star}$ \\
\hline
\end{tabular}

Atomic displacement parameters $\left(\AA^{2}\right)$ for $(\mathbf{4}) \cdot 1.5 \mathrm{C}_{8} \mathrm{H}_{10}$

\begin{tabular}{|l|l|l|l|l|l|l|}
\hline & $U^{\prime 1}$ & $U^{22}$ & $U^{\beta 3}$ & $U^{12}$ & $U^{13}$ & $U^{23}$ \\
\hline Os1 & $\begin{array}{l}0.01122 \\
(8)\end{array}$ & $\begin{array}{l}0.01568 \\
(8)\end{array}$ & $\begin{array}{l}0.01396 \\
(9)\end{array}$ & $\begin{array}{l}0.00621 \\
(6)\end{array}$ & $\begin{array}{l}0.00354 \\
(6)\end{array}$ & $\begin{array}{l}0.00397 \\
(6)\end{array}$ \\
\hline Os2 & $\begin{array}{l}0.01186 \\
(8)\end{array}$ & $\begin{array}{l}0.01575 \\
(8)\end{array}$ & $\begin{array}{l}0.01351 \\
(9)\end{array}$ & $\begin{array}{l}0.00615 \\
(6)\end{array}$ & $\begin{array}{l}0.00287 \\
(6)\end{array}$ & $\begin{array}{l}0.00414 \\
(6)\end{array}$ \\
\hline P1 & $0.0121(4)$ & $0.0176(4)$ & $0.0184(4)$ & $0.0067(3)$ & $0.0049(3)$ & $0.0049(3)$ \\
\hline P2 & $0.0138(4)$ & $0.0166(4)$ & $0.0154(4)$ & $0.0067(3)$ & $0.0037(3)$ & $0.0043(3)$ \\
\hline O1 & 0.0222 & 0.0301 & 0.0351 & 0.0158 & 0.0052 & 0.0158 \\
\hline O2 & $(13)$ & $(14)$ & $(16)$ & $(12)$ & $(11)$ & $(12)$ \\
\hline O3 & 0.0272 & 0.0324 & 0.0188 & 0.0140 & 0.0083 & 0.0012 \\
& $(14)$ & $(14)$ & $(15)$ & $(12)$ & $(11)$ & $(11)$ \\
\hline O4 & 0.0202 & 0.0274 & 0.0380 & 0.0134 & -0.0026 & 0.0088 \\
& $(13)$ & $(14)$ & $(17)$ & $(12)$ & $(11)$ & $(12)$ \\
\hline O5 & 0.0268 & 0.0426 & 0.0212 & 0.0132 & 0.0104 & 0.0090 \\
\hline & $(14)$ & $(17)$ & $(16)$ & $(13)$ & $(11)$ & $(12)$ \\
\hline
\end{tabular}




\begin{tabular}{|c|c|c|c|c|c|c|}
\hline & (12) & (13) & (13) & (10) & (10) & (10) \\
\hline O6 & $\begin{array}{l}0.0234 \\
(13)\end{array}$ & $\begin{array}{l}0.0257 \\
(13)\end{array}$ & $\begin{array}{l}0.0140 \\
(13)\end{array}$ & $\begin{array}{l}0.0054 \\
(11)\end{array}$ & $\begin{array}{l}0.0074 \\
(10)\end{array}$ & $\begin{array}{l}0.0013 \\
(10)\end{array}$ \\
\hline $\mathrm{O} 7$ & $\begin{array}{l}0.0134 \\
(11)\end{array}$ & $\begin{array}{l}0.0230 \\
(13)\end{array}$ & $\begin{array}{l}0.0245 \\
(14)\end{array}$ & $\begin{array}{l}0.0094 \\
(10)\end{array}$ & $\begin{array}{l}0.0022 \\
(10)\end{array}$ & $\begin{array}{l}0.0080 \\
(10)\end{array}$ \\
\hline $\mathrm{O} 8$ & $\begin{array}{l}0.0157 \\
(12)\end{array}$ & $\begin{array}{l}0.0193 \\
(12)\end{array}$ & $\begin{array}{l}0.0271 \\
(14)\end{array}$ & $\begin{array}{l}0.0084 \\
(10)\end{array}$ & $\begin{array}{l}0.0037 \\
(10)\end{array}$ & $\begin{array}{l}0.0050 \\
(10)\end{array}$ \\
\hline C1 & $\begin{array}{l}0.0131 \\
(16)\end{array}$ & $\begin{array}{l}0.0185 \\
(17)\end{array}$ & $\begin{array}{l}0.0234 \\
(19)\end{array}$ & $\begin{array}{l}0.0010 \\
(14)\end{array}$ & $\begin{array}{l}0.0085 \\
(14)\end{array}$ & $\begin{array}{l}0.0019 \\
(14)\end{array}$ \\
\hline C2 & $\begin{array}{l}0.0126 \\
(16)\end{array}$ & $\begin{array}{l}0.0211 \\
(17)\end{array}$ & $0.023(2)$ & $\begin{array}{l}0.0071 \\
(14)\end{array}$ & $\begin{array}{l}0.0014 \\
(14)\end{array}$ & $\begin{array}{l}0.0098 \\
(15)\end{array}$ \\
\hline C3 & $\begin{array}{l}0.0174 \\
(17)\end{array}$ & $\begin{array}{l}0.0176 \\
(16)\end{array}$ & $\begin{array}{l}0.0191 \\
(19)\end{array}$ & $\begin{array}{l}0.0020 \\
(14)\end{array}$ & $\begin{array}{l}0.0032 \\
(14)\end{array}$ & $\begin{array}{l}0.0025 \\
(14)\end{array}$ \\
\hline C4 & $\begin{array}{l}0.0156 \\
(17)\end{array}$ & $\begin{array}{l}0.0235 \\
(18)\end{array}$ & 0.017 (2) & $\begin{array}{l}0.0046 \\
(14)\end{array}$ & $\begin{array}{l}0.0029 \\
(14)\end{array}$ & $\begin{array}{l}0.0058 \\
(14)\end{array}$ \\
\hline C5 & $\begin{array}{l}0.0222 \\
(19)\end{array}$ & $\begin{array}{l}0.0252 \\
(18)\end{array}$ & $0.023(2)$ & $\begin{array}{l}0.0146 \\
(16)\end{array}$ & $\begin{array}{l}0.0113 \\
(15)\end{array}$ & $\begin{array}{l}0.0100 \\
(15)\end{array}$ \\
\hline C6 & $0.031(2)$ & $0.037(2)$ & $0.020(2)$ & $\begin{array}{l}0.0171 \\
(18)\end{array}$ & $\begin{array}{l}0.0108 \\
(16)\end{array}$ & $\begin{array}{l}0.0061 \\
(16)\end{array}$ \\
\hline $\mathrm{C7}$ & $\begin{array}{l}0.0218 \\
(18)\end{array}$ & $\begin{array}{l}0.0230 \\
(18)\end{array}$ & $\begin{array}{l}0.0199 \\
(19)\end{array}$ & $\begin{array}{l}0.0101 \\
(15)\end{array}$ & $\begin{array}{l}0.0055 \\
(15)\end{array}$ & $\begin{array}{l}0.0066 \\
(14)\end{array}$ \\
\hline C8 & $0.023(2)$ & $0.027(2)$ & $0.050(3)$ & $\begin{array}{l}0.0144 \\
(17)\end{array}$ & $\begin{array}{l}0.0009 \\
(18)\end{array}$ & $\begin{array}{l}0.0137 \\
(19)\end{array}$ \\
\hline C9 & $\begin{array}{l}0.0133 \\
(16)\end{array}$ & $\begin{array}{l}0.0208 \\
(17)\end{array}$ & $\begin{array}{l}0.0197 \\
(19)\end{array}$ & $\begin{array}{l}0.0060 \\
(13)\end{array}$ & $\begin{array}{l}0.0041 \\
(13)\end{array}$ & $\begin{array}{l}0.0056 \\
(14)\end{array}$ \\
\hline C10 & $\begin{array}{l}0.0162 \\
(16)\end{array}$ & $\begin{array}{l}0.0210 \\
(17)\end{array}$ & $\begin{array}{l}0.0190 \\
(18)\end{array}$ & $\begin{array}{l}0.0073 \\
(14)\end{array}$ & $\begin{array}{l}0.0005 \\
(13)\end{array}$ & $\begin{array}{l}0.0048 \\
(14)\end{array}$ \\
\hline C11 & $\begin{array}{l}0.0190 \\
(17)\end{array}$ & $\begin{array}{l}0.0219 \\
(17)\end{array}$ & $0.026(2)$ & $\begin{array}{l}0.0100 \\
(15)\end{array}$ & $\begin{array}{l}0.0059 \\
(15)\end{array}$ & $\begin{array}{l}0.0037 \\
(15)\end{array}$ \\
\hline
\end{tabular}




\begin{tabular}{|c|c|c|c|c|c|c|}
\hline C12 & $\begin{array}{l}0.0163 \\
(17)\end{array}$ & $\begin{array}{l}0.0267 \\
(18)\end{array}$ & $0.031(2)$ & $\begin{array}{l}0.0108 \\
(15)\end{array}$ & $\begin{array}{l}0.0076 \\
(15)\end{array}$ & $\begin{array}{l}0.0160 \\
(16)\end{array}$ \\
\hline $\mathrm{C} 13$ & $\begin{array}{l}0.0201 \\
(18)\end{array}$ & $0.039(2)$ & $0.028(2)$ & $\begin{array}{l}0.0150 \\
(17)\end{array}$ & $\begin{array}{l}0.0090 \\
(15)\end{array}$ & $\begin{array}{l}0.0173 \\
(17)\end{array}$ \\
\hline C14 & $\begin{array}{l}0.0200 \\
(18)\end{array}$ & $0.032(2)$ & $0.021(2)$ & $\begin{array}{l}0.0131 \\
(16)\end{array}$ & $\begin{array}{l}0.0069 \\
(15)\end{array}$ & $\begin{array}{l}0.0083 \\
(15)\end{array}$ \\
\hline C15 & $0.028(2)$ & $0.034(2)$ & $0.040(3)$ & $\begin{array}{l}0.0198 \\
(18)\end{array}$ & $\begin{array}{l}0.0094 \\
(18)\end{array}$ & $\begin{array}{l}0.0157 \\
(19)\end{array}$ \\
\hline C16 & $\begin{array}{l}0.0146 \\
(16)\end{array}$ & $\begin{array}{l}0.0218 \\
(17)\end{array}$ & $\begin{array}{l}0.0185 \\
(18)\end{array}$ & $\begin{array}{l}0.0073 \\
(14)\end{array}$ & $\begin{array}{l}0.0050 \\
(13)\end{array}$ & $\begin{array}{l}0.0068 \\
(14)\end{array}$ \\
\hline C17 & $\begin{array}{l}0.0167 \\
(18)\end{array}$ & $\begin{array}{l}0.0278 \\
(19)\end{array}$ & $0.025(2)$ & $\begin{array}{l}0.0114 \\
(15)\end{array}$ & $\begin{array}{l}0.0062 \\
(15)\end{array}$ & $\begin{array}{l}0.0060 \\
(15)\end{array}$ \\
\hline C18 & $\begin{array}{l}0.0126 \\
(17)\end{array}$ & $\begin{array}{l}0.0299 \\
(19)\end{array}$ & $0.023(2)$ & $\begin{array}{l}0.0060 \\
(15)\end{array}$ & $\begin{array}{l}0.0019 \\
(14)\end{array}$ & $\begin{array}{l}0.0045 \\
(15)\end{array}$ \\
\hline C19 & $\begin{array}{l}0.0199 \\
(17)\end{array}$ & $\begin{array}{l}0.0196 \\
(17)\end{array}$ & $\begin{array}{l}0.0193 \\
(19)\end{array}$ & $\begin{array}{l}0.0071 \\
(14)\end{array}$ & $\begin{array}{l}0.0071 \\
(14)\end{array}$ & $\begin{array}{l}0.0068 \\
(14)\end{array}$ \\
\hline C20 & $\begin{array}{l}0.0175 \\
(17)\end{array}$ & $\begin{array}{l}0.0230 \\
(18)\end{array}$ & $0.024(2)$ & $\begin{array}{l}0.0106 \\
(14)\end{array}$ & $\begin{array}{l}0.0063 \\
(14)\end{array}$ & $\begin{array}{l}0.0072 \\
(15)\end{array}$ \\
\hline $\mathrm{C} 21$ & $\begin{array}{l}0.0141 \\
(16)\end{array}$ & $\begin{array}{l}0.0229 \\
(18)\end{array}$ & $\begin{array}{l}0.0199 \\
(19)\end{array}$ & $\begin{array}{l}0.0044 \\
(14)\end{array}$ & $\begin{array}{l}0.0011 \\
(14)\end{array}$ & $\begin{array}{l}0.0042 \\
(14)\end{array}$ \\
\hline $\mathrm{C} 22$ & $\begin{array}{l}0.0226 \\
(18)\end{array}$ & $\begin{array}{l}0.0245 \\
(18)\end{array}$ & $0.023(2)$ & $\begin{array}{l}0.0087 \\
(15)\end{array}$ & $\begin{array}{l}0.0041 \\
(15)\end{array}$ & $\begin{array}{l}0.0003 \\
(15)\end{array}$ \\
\hline $\mathrm{C} 23$ & $\begin{array}{l}0.0177 \\
(17)\end{array}$ & $\begin{array}{l}0.0211 \\
(17)\end{array}$ & $\begin{array}{l}0.0208 \\
(19)\end{array}$ & $\begin{array}{l}0.0108 \\
(14)\end{array}$ & $\begin{array}{l}0.0093 \\
(14)\end{array}$ & $\begin{array}{l}0.0072 \\
(14)\end{array}$ \\
\hline $\mathrm{C} 24$ & $\begin{array}{l}0.0209 \\
(18)\end{array}$ & $\begin{array}{l}0.0240 \\
(18)\end{array}$ & $0.024(2)$ & $\begin{array}{l}0.0088 \\
(15)\end{array}$ & $\begin{array}{l}0.0070 \\
(15)\end{array}$ & $\begin{array}{l}0.0043 \\
(15)\end{array}$ \\
\hline $\mathrm{C} 25$ & $\begin{array}{l}0.0166 \\
(17)\end{array}$ & $\begin{array}{l}0.0230 \\
(18)\end{array}$ & $0.031(2)$ & $\begin{array}{l}0.0074 \\
(14)\end{array}$ & $\begin{array}{l}0.0119 \\
(15)\end{array}$ & $\begin{array}{l}0.0072 \\
(15)\end{array}$ \\
\hline C26 & 0.0280 & 0.0201 & $0.029(2)$ & 0.0137 & 0.0144 & 0.0113 \\
\hline
\end{tabular}




\begin{tabular}{|c|c|c|c|c|c|c|}
\hline & (19) & (17) & & (15) & (16) & (15) \\
\hline $\mathrm{C} 27$ & $0.026(2)$ & $\begin{array}{l}0.0286 \\
(19)\end{array}$ & $0.023(2)$ & $\begin{array}{l}0.0101 \\
(16)\end{array}$ & $\begin{array}{l}0.0044 \\
(16)\end{array}$ & $\begin{array}{l}0.0095 \\
(16)\end{array}$ \\
\hline $\mathrm{C} 28$ & $\begin{array}{l}0.0196 \\
(18)\end{array}$ & $\begin{array}{l}0.0251 \\
(18)\end{array}$ & $0.025(2)$ & $\begin{array}{l}0.0059 \\
(15)\end{array}$ & $\begin{array}{l}0.0037 \\
(15)\end{array}$ & $\begin{array}{l}0.0106 \\
(15)\end{array}$ \\
\hline C29 & $0.037(2)$ & $0.031(2)$ & $0.031(2)$ & $\begin{array}{l}0.0121 \\
(18)\end{array}$ & $\begin{array}{l}0.0162 \\
(18)\end{array}$ & $\begin{array}{l}0.0127 \\
(18)\end{array}$ \\
\hline C30 & $\begin{array}{l}0.0192 \\
(17)\end{array}$ & $\begin{array}{l}0.0187 \\
(16)\end{array}$ & $\begin{array}{l}0.0175 \\
(18)\end{array}$ & $\begin{array}{l}0.0107 \\
(14)\end{array}$ & $\begin{array}{l}0.0031 \\
(14)\end{array}$ & $\begin{array}{l}0.0067 \\
(13)\end{array}$ \\
\hline C31 & $\begin{array}{l}0.0164 \\
(17)\end{array}$ & $\begin{array}{l}0.0204 \\
(17)\end{array}$ & $\begin{array}{l}0.0229 \\
(19)\end{array}$ & $\begin{array}{l}0.0090 \\
(14)\end{array}$ & $\begin{array}{l}0.0036 \\
(14)\end{array}$ & $\begin{array}{l}0.0065 \\
(14)\end{array}$ \\
\hline C32 & $\begin{array}{l}0.0233 \\
(18)\end{array}$ & $\begin{array}{l}0.0190 \\
(17)\end{array}$ & $0.022(2)$ & $\begin{array}{l}0.0100 \\
(14)\end{array}$ & $\begin{array}{l}0.0089 \\
(15)\end{array}$ & $\begin{array}{l}0.0066 \\
(14)\end{array}$ \\
\hline C33 & $0.030(2)$ & $\begin{array}{l}0.0171 \\
(16)\end{array}$ & $\begin{array}{l}0.0207 \\
(19)\end{array}$ & $\begin{array}{l}0.0124 \\
(15)\end{array}$ & $\begin{array}{l}0.0058 \\
(15)\end{array}$ & $\begin{array}{l}0.0062 \\
(14)\end{array}$ \\
\hline C34 & $\begin{array}{l}0.0185 \\
(17)\end{array}$ & $\begin{array}{l}0.0223 \\
(17)\end{array}$ & $0.023(2)$ & $\begin{array}{l}0.0081 \\
(14)\end{array}$ & $\begin{array}{l}-0.0007 \\
(14)\end{array}$ & $\begin{array}{l}0.0032 \\
(14)\end{array}$ \\
\hline C35 & $\begin{array}{l}0.0177 \\
(17)\end{array}$ & $\begin{array}{l}0.0231 \\
(17)\end{array}$ & $\begin{array}{l}0.0209 \\
(19)\end{array}$ & $\begin{array}{l}0.0103 \\
(14)\end{array}$ & $\begin{array}{l}0.0035 \\
(14)\end{array}$ & $\begin{array}{l}0.0045 \\
(14)\end{array}$ \\
\hline C36 & $0.033(2)$ & $0.032(2)$ & $0.022(2)$ & $\begin{array}{l}0.0176 \\
(18)\end{array}$ & $\begin{array}{l}0.0048 \\
(16)\end{array}$ & $\begin{array}{l}0.0047 \\
(16)\end{array}$ \\
\hline C37 & $\begin{array}{l}0.0149 \\
(16)\end{array}$ & $\begin{array}{l}0.0175 \\
(16)\end{array}$ & $\begin{array}{l}0.0180 \\
(18)\end{array}$ & $\begin{array}{l}0.0073 \\
(13)\end{array}$ & $\begin{array}{l}0.0035 \\
(13)\end{array}$ & $\begin{array}{l}0.0016 \\
(13)\end{array}$ \\
\hline C38 & $\begin{array}{l}0.0202 \\
(18)\end{array}$ & $\begin{array}{l}0.0263 \\
(19)\end{array}$ & $0.029(2)$ & $\begin{array}{l}0.0111 \\
(15)\end{array}$ & $\begin{array}{l}0.0103 \\
(15)\end{array}$ & $\begin{array}{l}0.0133 \\
(16)\end{array}$ \\
\hline C39 & $\begin{array}{l}0.0199 \\
(18)\end{array}$ & $\begin{array}{l}0.0278 \\
(19)\end{array}$ & $0.030(2)$ & $\begin{array}{l}0.0145 \\
(15)\end{array}$ & $\begin{array}{l}0.0080 \\
(15)\end{array}$ & $\begin{array}{l}0.0077 \\
(16)\end{array}$ \\
\hline $\mathrm{C} 40$ & $\begin{array}{l}0.0209 \\
(18)\end{array}$ & $0.036(2)$ & $0.024(2)$ & $\begin{array}{l}0.0134 \\
(16)\end{array}$ & $\begin{array}{l}0.0091 \\
(15)\end{array}$ & $\begin{array}{l}0.0105 \\
(16)\end{array}$ \\
\hline
\end{tabular}




\begin{tabular}{|c|c|c|c|c|c|c|}
\hline C41 & $\begin{array}{l}0.0228 \\
(19)\end{array}$ & $0.033(2)$ & $0.037(2)$ & $\begin{array}{l}0.0153 \\
(16)\end{array}$ & $\begin{array}{l}0.0159 \\
(17)\end{array}$ & $\begin{array}{l}0.0217 \\
(18)\end{array}$ \\
\hline C42 & $\begin{array}{l}0.0215 \\
(18)\end{array}$ & $\begin{array}{l}0.0241 \\
(18)\end{array}$ & $0.033(2)$ & $\begin{array}{l}0.0122 \\
(15)\end{array}$ & $\begin{array}{l}0.0097 \\
(16)\end{array}$ & $\begin{array}{l}0.0127 \\
(16)\end{array}$ \\
\hline C43 & $0.029(2)$ & $0.047(2)$ & $0.037(2)$ & $\begin{array}{l}0.0218 \\
(19)\end{array}$ & $\begin{array}{l}0.0172 \\
(18)\end{array}$ & $0.019(2)$ \\
\hline C44 & $\begin{array}{l}0.0122 \\
(15)\end{array}$ & $\begin{array}{l}0.0193 \\
(16)\end{array}$ & $\begin{array}{l}0.0219 \\
(19)\end{array}$ & $\begin{array}{l}0.0075 \\
(13)\end{array}$ & $\begin{array}{l}0.0068 \\
(13)\end{array}$ & $\begin{array}{l}0.0079 \\
(14)\end{array}$ \\
\hline C45 & $\begin{array}{l}0.0166 \\
(17)\end{array}$ & $\begin{array}{l}0.0223 \\
(17)\end{array}$ & $\begin{array}{l}0.0213 \\
(19)\end{array}$ & $\begin{array}{l}0.0086 \\
(14)\end{array}$ & $\begin{array}{l}0.0073 \\
(14)\end{array}$ & $\begin{array}{l}0.0077 \\
(14)\end{array}$ \\
\hline C46 & $\begin{array}{l}0.0250 \\
(19)\end{array}$ & $\begin{array}{l}0.0189 \\
(17)\end{array}$ & $0.031(2)$ & $\begin{array}{l}0.0104 \\
(15)\end{array}$ & $\begin{array}{l}0.0122 \\
(16)\end{array}$ & $\begin{array}{l}0.0091 \\
(15)\end{array}$ \\
\hline C47 & $\begin{array}{l}0.0186 \\
(18)\end{array}$ & $\begin{array}{l}0.0278 \\
(19)\end{array}$ & $0.032(2)$ & $\begin{array}{l}0.0110 \\
(15)\end{array}$ & $\begin{array}{l}0.0123 \\
(15)\end{array}$ & $\begin{array}{l}0.0156 \\
(16)\end{array}$ \\
\hline C48 & $\begin{array}{l}0.0199 \\
(18)\end{array}$ & $0.030(2)$ & $0.025(2)$ & $\begin{array}{l}0.0095 \\
(15)\end{array}$ & $\begin{array}{l}0.0067 \\
(15)\end{array}$ & $\begin{array}{l}0.0146 \\
(16)\end{array}$ \\
\hline C49 & $\begin{array}{l}0.0203 \\
(18)\end{array}$ & $\begin{array}{l}0.0247 \\
(18)\end{array}$ & $\begin{array}{l}0.0206 \\
(19)\end{array}$ & $\begin{array}{l}0.0104 \\
(15)\end{array}$ & $\begin{array}{l}0.0081 \\
(14)\end{array}$ & $\begin{array}{l}0.0075 \\
(14)\end{array}$ \\
\hline C50 & $0.035(2)$ & $0.037(2)$ & $0.046(3)$ & $\begin{array}{l}0.0227 \\
(19)\end{array}$ & $0.015(2)$ & $0.024(2)$ \\
\hline C51 & $0.024(2)$ & $0.035(2)$ & $0.035(2)$ & $\begin{array}{l}0.0179 \\
(17)\end{array}$ & $\begin{array}{l}0.0029 \\
(17)\end{array}$ & $\begin{array}{l}0.0131 \\
(18)\end{array}$ \\
\hline C52 & $\begin{array}{l}0.0186 \\
(19)\end{array}$ & $0.027(2)$ & $0.042(3)$ & $\begin{array}{l}0.0088 \\
(16)\end{array}$ & $\begin{array}{l}0.0020 \\
(17)\end{array}$ & $\begin{array}{l}0.0077 \\
(18)\end{array}$ \\
\hline C53 & $0.024(2)$ & $0.030(2)$ & $0.040(3)$ & $\begin{array}{l}0.0174 \\
(17)\end{array}$ & $\begin{array}{l}0.0077 \\
(17)\end{array}$ & $\begin{array}{l}0.0066 \\
(18)\end{array}$ \\
\hline C54 & $\begin{array}{l}0.0182 \\
(18)\end{array}$ & $0.031(2)$ & $0.032(2)$ & $\begin{array}{l}0.0139 \\
(16)\end{array}$ & $\begin{array}{l}-0.0015 \\
(15)\end{array}$ & $\begin{array}{l}0.0118 \\
(17)\end{array}$ \\
\hline C55 & $0.028(2)$ & 0.0253 & $0.028(2)$ & 0.0117 & -0.0011 & 0.0077 \\
\hline
\end{tabular}




\begin{tabular}{|l|l|l|l|l|l|l|}
\hline & & $(19)$ & & $(16)$ & $(16)$ & $(16)$ \\
\hline C56 & $0.034(2)$ & $0.031(2)$ & $0.025(2)$ & $\begin{array}{l}0.0188 \\
(18)\end{array}$ & $\begin{array}{l}0.0002 \\
(17)\end{array}$ & $\begin{array}{l}0.0051 \\
(17)\end{array}$ \\
\hline C57 & $0.029(2)$ & $0.045(3)$ & $0.045(3)$ & $0.019(2)$ & $0.013(2)$ & $0.015(2)$ \\
\hline C58 & $0.027(2)$ & $0.033(2)$ & $0.049(3)$ & $\begin{array}{l}0.0144 \\
(18)\end{array}$ & $\begin{array}{l}0.0086 \\
(19)\end{array}$ & $0.017(2)$ \\
\hline C59 & $0.032(2)$ & $0.028(2)$ & $0.090(5)$ & $\begin{array}{l}0.0139 \\
(19)\end{array}$ & $0.025(3)$ & $0.018(2)$ \\
\hline C60 & $0.049(3)$ & $0.027(2)$ & $0.088(4)$ & $0.020(2)$ & $0.047(3)$ & $0.024(2)$ \\
\hline C61 & $0.050(3)$ & $0.027(2)$ & $0.062(3)$ & $0.019(2)$ & $0.027(2)$ & $0.014(2)$ \\
\hline C62 & $0.094(5)$ & $0.043(3)$ & $0.075(5)$ & $0.029(3)$ & $0.036(4)$ & $0.014(3)$ \\
\hline
\end{tabular}

Geometric parameters $\left(\AA \stackrel{\AA}{\circ} \stackrel{\circ}{)}\right.$ for $(4) \cdot 1.5 C_{8} H_{10}$

\begin{tabular}{|c|c|c|c|}
\hline Os1-Os2 & $2.7534(2)$ & $\mathrm{C} 29-\mathrm{H} 29 \mathrm{~A}$ & 0.9600 \\
\hline Os1-P1 & $2.4123(8)$ & $\mathrm{C} 29-\mathrm{H} 29 \mathrm{~B}$ & 0.9600 \\
\hline Os1-O5 & $2.132(2)$ & $\mathrm{C} 29-\mathrm{H} 29 \mathrm{C}$ & 0.9600 \\
\hline Os1-O7 & $2.131(2)$ & $\mathrm{C} 30-\mathrm{C} 31$ & $1.402(5)$ \\
\hline Os $1-\mathrm{C} 1$ & $1.852(4)$ & C30-C35 & $1.404(5)$ \\
\hline Os1-C2 & $1.857(4)$ & $\mathrm{C} 31-\mathrm{H} 31$ & 0.9300 \\
\hline Os2-P2 & $2.4233(8)$ & C31-C32 & $1.382(5)$ \\
\hline Os2-O6 & 2.129 (3) & $\mathrm{C} 32-\mathrm{H} 32$ & 0.9300 \\
\hline Os2-O8 & $2.135(2)$ & C32-C33 & $1.395(5)$ \\
\hline Os2-C3 & $1.857(4)$ & C33-C34 & $1.391(6)$ \\
\hline Os2-C4 & $1.853(4)$ & C33-C36 & 1.507 (5) \\
\hline P1-C9 & $1.821(4)$ & C34-H34 & 0.9300 \\
\hline $\mathrm{P} 1-\mathrm{C} 16$ & $1.829(4)$ & C34-C35 & $1.386(6)$ \\
\hline
\end{tabular}




\begin{tabular}{|l|l|l|l|}
\hline P1—C23 & $1.836(4)$ & C35-H35 & 0.9300 \\
\hline P2—C30 & $1.828(4)$ & C36-H36A & 0.9600 \\
\hline P2-C37 & $1.826(4)$ & C36-H36B & 0.9600 \\
\hline P2-C44 & $1.836(4)$ & C36-H36C & 0.9600 \\
\hline O1-C1 & $1.161(5)$ & C37-C38 & $1.399(5)$ \\
\hline O2-C2 & $1.151(5)$ & C37-C42 & $1.395(5)$ \\
\hline O3-C3 & $1.153(5)$ & C38-H38 & 0.9300 \\
\hline O4-C4 & $1.149(5)$ & C38-C39 & $1.390(6)$ \\
\hline O5-C5 & $1.265(5)$ & C39-H39 & 0.9300 \\
\hline O6-C5 & $1.253(5)$ & C39-C40 & $1.389(6)$ \\
\hline O7-C7 & $1.257(5)$ & C40-C41 & $1.386(6)$ \\
\hline O8-C7 & $1.259(5)$ & C40-C43 & $1.511(6)$ \\
\hline C5-C6 & $1.495(5)$ & C41-H41 & 0.9300 \\
\hline C6-H6A & 0.9600 & C41-C42 & $1.390(6)$ \\
\hline C6-H6B & 0.9600 & C42-H42 & 0.9300 \\
\hline C6-H6C & 0.9600 & C43-H43A & 0.9600 \\
\hline C7—C8 & $1.501(5)$ & C43-H43B & 0.9600 \\
\hline C8-H8A & 0.9600 & C43-H43C & 0.9600 \\
\hline C8-H8B & 0.9600 & C44-C45 & $1.394(5)$ \\
\hline C8-H8C & 0.9600 & C44-C49 & $1.400(5)$ \\
\hline C9-C10 & $1.398(5)$ & C45-H45 & 0.9300 \\
\hline C9-C14 & $1.398(5)$ & C45-C46 & $1.384(5)$ \\
\hline C10-H10 & 0.9300 & C46-H46 & 0.9300 \\
\hline C10-C11 & $1.381(5)$ & C46-C47 & $1.390(6)$ \\
\hline C11-H11 & 0.9300 & $1.395(6)$ \\
\hline
\end{tabular}




\begin{tabular}{|l|l|l|l|}
\hline C11-C12 & $1.401(6)$ & C47-C50 & $1.499(5)$ \\
\hline C12-C13 & $1.385(6)$ & C48-H48 & 0.9300 \\
\hline C12-C15 & $1.507(5)$ & C48-C49 & $1.382(5)$ \\
\hline C13-H13 & 0.9300 & C49-H49 & 0.9300 \\
\hline C13-C14 & $1.385(6)$ & C50-H50A & 0.9600 \\
\hline C14-H14 & 0.9300 & C50-H50B & 0.9600 \\
\hline C15-H15A & 0.9600 & C50-H50C & 0.9600 \\
\hline C15-H15B & 0.9600 & C51-C52 & $1.391(7)$ \\
\hline C15-H15C & 0.9600 & C51-C56 & $1.389(6)$ \\
\hline C16-C17 & $1.400(5)$ & C51-C57 & $1.516(6)$ \\
\hline C16-C21 & $1.389(5)$ & C52-H52 & 0.9300 \\
\hline C17-H17 & 0.9300 & C52-C53 & $1.397(6)$ \\
\hline C17-C18 & $1.385(6)$ & C53-H53 & 0.9300 \\
\hline C18-H18 & 0.9300 & C53-C54 & $1.388(6)$ \\
\hline C18-C19 & $1.393(5)$ & C54-C55 & $1.392(6)$ \\
\hline C19-C20 & $1.403(5)$ & C54-C58 & $1.509(6)$ \\
\hline C19-C22 & $1.510(5)$ & C55-H55 & 0.9300 \\
\hline C20-H20 & 0.9300 & C55-C56 & $1.386(6)$ \\
\hline C20-C21 & $1.377(5)$ & C56-H56 & 0.9300 \\
\hline C21-H21 & 0.9300 & C57-H57A & 0.9600 \\
\hline C22-H22A & 0.9600 & C57-H57B & 0.9600 \\
\hline C22-H22B & 0.9600 & C57-H57C & 0.9600 \\
\hline C22-H22C & 0.9600 & C58-H58A & 0.9600 \\
\hline C23-C24 & $1.393(5)$ & C58-H58B & 0.9600 \\
\hline C23-C28 & $1.380(5)$ & C58-H58C & 0.9600 \\
\hline
\end{tabular}




\begin{tabular}{|l|l|l|l|}
\hline C24-H24 & 0.9300 & C59-H59 & 0.9300 \\
\hline C24-C25 & $1.389(5)$ & C59-C60 & $1.371(9)$ \\
\hline C25-H25 & 0.9300 & C59-C61 & $1.392(7)$ \\
\hline C25-C26 & $1.390(6)$ & C60-H60 & 0.9300 \\
\hline C26-C27 & $1.389(5)$ & C60-C61 & $1.377(8)$ \\
\hline C26-C29 & $1.515(5)$ & C61-C62 & $1.501(9)$ \\
\hline C27-H27 & 0.9300 & C62-H62A & 0.9600 \\
\hline C27-C28 & $1.397(6)$ & C62-H62B & 0.9600 \\
\hline C28-H28 & 0.9300 & C62-H62C & 0.9600 \\
\hline P1-Os1-Os2 & $162.49(2)$ & C26-C27-C28 & $121.4(4)$ \\
\hline O5-Os1-Os2 & $81.65(7)$ & C28-C27-H27 & 119.3 \\
\hline O5-Os1-P1 & $83.89(7)$ & C23-C28-C27 & $120.0(4)$ \\
\hline O7-Os1-Os2 & $82.78(6)$ & C23-C28-H28 & 120.0 \\
\hline O7-Os1-P1 & $85.94(7)$ & C27-C28-H28 & 120.0 \\
\hline O7-Os1-O5 & $83.98(10)$ & C26-C29-H29A & 109.5 \\
\hline C1-Os1-Os2 & $93.70(10)$ & C26-C29-H29B & 109.5 \\
\hline C1-Os1-P1 & $97.17(10)$ & C26-C29-H29C & 109.5 \\
\hline C1-Os1-O5 & $94.24(13)$ & $\begin{array}{l}\text { H29A-C29- } \\
\text { H29B }\end{array}$ & 109.5 \\
\hline C1-Os1-O7 & $176.25(12)$ & $\begin{array}{l}\text { H29A-C29- } \\
\text { H29C }\end{array}$ & 109.5 \\
\hline C1-Os1-C2 & $91.78(16)$ & $\begin{array}{l}\text { H29B-C29- } \\
\text { H29C }\end{array}$ & 109.5 \\
\hline C2-Os1-Os2 & $96.48(10)$ & C31-C30-P2 & $118.5(3)$ \\
\hline Os1-P1 & $96.86(11)$ & C31-C30-C35 & $118.2(3)$ \\
\hline
\end{tabular}




\begin{tabular}{|c|c|c|c|}
\hline C2-Os1-O5 & $173.80(12)$ & $\mathrm{C} 35-\mathrm{C} 30-\mathrm{P} 2$ & $123.3(3)$ \\
\hline $\mathrm{C} 2-\mathrm{Os} 1-\mathrm{O} 7$ & $89.93(13)$ & $\mathrm{C} 30-\mathrm{C} 31-\mathrm{H} 31$ & 119.7 \\
\hline $\mathrm{P} 2-\mathrm{Os} 2-\mathrm{O} 1$ & $165.16(2)$ & C32-C31-C30 & $120.5(3)$ \\
\hline O6-Os2-Os1 & $82.41(7)$ & $\mathrm{C} 32-\mathrm{C} 31-\mathrm{H} 31$ & 119.7 \\
\hline O6-Os2-P2 & $87.53(7)$ & $\mathrm{C} 31-\mathrm{C} 32-\mathrm{H} 32$ & 119.2 \\
\hline O6-Os2-O8 & $83.13(11)$ & C31-C32-C33 & $121.6(4)$ \\
\hline O8-Os2-Os1 & $81.09(7)$ & C33-C32-H32 & 119.2 \\
\hline O8-Os2-P2 & $86.92(7)$ & $\mathrm{C} 32-\mathrm{C} 33-\mathrm{C} 36$ & $121.0(4)$ \\
\hline C3-Os2-Os1 & $94.75(10)$ & C34-C33-C32 & $117.7(4)$ \\
\hline C3-Os2-P2 & $96.72(11)$ & $\mathrm{C} 34-\mathrm{C} 33-\mathrm{C} 36$ & $121.3(4)$ \\
\hline $\mathrm{C} 3-\mathrm{Os} 2-\mathrm{O} 6$ & 93.37 (14) & $\mathrm{C} 33-\mathrm{C} 34-\mathrm{H} 34$ & 119.1 \\
\hline C3-Os2-O8 & $174.87(13)$ & C35-C34-C33 & $121.7(3)$ \\
\hline $\mathrm{C} 4-\mathrm{Os} 2-\mathrm{Os} 1$ & $94.51(11)$ & C35-C34-H34 & 119.1 \\
\hline C4-Os2-P2 & $95.24(11)$ & $\mathrm{C} 30-\mathrm{C} 35-\mathrm{H} 35$ & 119.9 \\
\hline C4-Os2-O6 & $176.67(12)$ & C34-C35-C30 & $120.3(4)$ \\
\hline C4-Os2-O8 & $95.15(14)$ & C34-C35-H35 & 119.9 \\
\hline $\mathrm{C} 4-\mathrm{O} 2-\mathrm{C} 3$ & $88.15(16)$ & C33-C36-H36A & 109.5 \\
\hline $\mathrm{C} 9-\mathrm{P} 1-\mathrm{Os} 1$ & $109.92(11)$ & C33-C36-H36B & 109.5 \\
\hline $\mathrm{C} 9-\mathrm{P} 1-\mathrm{C} 16$ & $107.47(16)$ & $\mathrm{C} 33-\mathrm{C} 36-\mathrm{H} 36 \mathrm{C}$ & 109.5 \\
\hline $\mathrm{C} 9-\mathrm{P} 1-\mathrm{C} 23$ & $101.55(16)$ & $\begin{array}{l}\text { H36A-C36- } \\
\text { H36B }\end{array}$ & 109.5 \\
\hline $\mathrm{C} 16-\mathrm{P} 1-\mathrm{Os} 1$ & $114.91(12)$ & $\begin{array}{l}\mathrm{H} 36 \mathrm{~A}-\mathrm{C} 36- \\
\mathrm{H} 36 \mathrm{C}\end{array}$ & 109.5 \\
\hline $\mathrm{C} 16-\mathrm{P} 1-\mathrm{C} 23$ & $102.87(16)$ & $\begin{array}{l}\mathrm{H} 36 \mathrm{~B}-\mathrm{C} 36- \\
\mathrm{H} 36 \mathrm{C}\end{array}$ & 109.5 \\
\hline $\mathrm{C} 23-\mathrm{P} 1-\mathrm{Os} 1$ & $118.80(12)$ & C38-C37-P2 & $119.1(3)$ \\
\hline
\end{tabular}




\begin{tabular}{|l|l|l|l|}
\hline C30-P2-Os2 & $115.60(12)$ & C42-C37-P2 & $122.8(3)$ \\
\hline C30-P2-C44 & $103.11(16)$ & C42-C37-C38 & $118.0(3)$ \\
\hline C37-P2-Os2 & $113.05(11)$ & C37-C38-H38 & 119.7 \\
\hline C37-P2-C30 & $105.73(16)$ & C39-C38-C37 & $120.6(4)$ \\
\hline C37-P2-C44 & $104.30(16)$ & C39-C38-H38 & 119.7 \\
\hline C44-P2-Os2 & $113.87(11)$ & C38-C39-H39 & 119.4 \\
\hline C5-O5-Os1 & $122.6(2)$ & C40-C39-C38 & $121.2(4)$ \\
\hline C5-O6-Os2 & $122.3(2)$ & C40-C39-H39 & 119.4 \\
\hline C7-O7-Os1 & $121.3(2)$ & C39-C40-C43 & $120.2(4)$ \\
\hline C7-O8-Os2 & $123.0(2)$ & C41-C40-C39 & $118.0(4)$ \\
\hline O1-C1-Os1 & $179.0(3)$ & C41-C40-C43 & $121.8(4)$ \\
\hline O2-C2-Os1 & $176.8(3)$ & C40-C41-H41 & 119.3 \\
\hline O3-C3-Os2 & $178.4(3)$ & C40-C41-C42 & $121.4(4)$ \\
\hline O4-C4-Os2 & $179.1(3)$ & C42-C41-H41 & 119.3 \\
\hline O5-C5-C6 & $116.4(3)$ & C37-C42-H42 & 119.7 \\
\hline O6-C5-O5 & $125.6(3)$ & C41-C42-C37 & $120.7(4)$ \\
\hline O6-C5-C6 & $118.0(3)$ & C41-C42-H42 & 119.7 \\
\hline C5-C6-H6A & 109.5 & C40-C43-H43A & 109.5 \\
\hline C5-C6-H6B & 109.5 & C40-C43-H43B & 109.5 \\
\hline C5-C6-H6C & 109.5 & C40-C43-H43C & 109.5 \\
\hline H6A-C6-H6B & 109.5 & H43A-C43- & 109.5 \\
\hline H6A-C6-H6C & 109.5 & H43B & 109.5 \\
\hline H6B-C6-H6C & 109.5 & H43C & 109.5 \\
\hline
\end{tabular}




\begin{tabular}{|c|c|c|c|}
\hline $\mathrm{O} 7-\mathrm{C} 7-\mathrm{O} 8$ & $125.7(3)$ & $\mathrm{C} 45-\mathrm{C} 44-\mathrm{P} 2$ & $123.6(3)$ \\
\hline O7-C7-C8 & $116.6(3)$ & C45-C44-C49 & $117.8(3)$ \\
\hline $\mathrm{O} 8-\mathrm{C} 7-\mathrm{C} 8$ & $117.7(3)$ & C49-C44-P2 & $118.5(3)$ \\
\hline $\mathrm{C} 7-\mathrm{C} 8-\mathrm{H} 8 \mathrm{~A}$ & 109.5 & $\mathrm{C} 44-\mathrm{C} 45-\mathrm{H} 45$ & 119.5 \\
\hline $\mathrm{C} 7-\mathrm{C} 8-\mathrm{H} 8 \mathrm{~B}$ & 109.5 & $\mathrm{C} 46-\mathrm{C} 45-\mathrm{C} 44$ & $121.0(3)$ \\
\hline $\mathrm{C} 7-\mathrm{C} 8-\mathrm{H} 8 \mathrm{C}$ & 109.5 & $\mathrm{C} 46-\mathrm{C} 45-\mathrm{H} 45$ & 119.5 \\
\hline $\mathrm{H} 8 \mathrm{~A}-\mathrm{C} 8-\mathrm{H} 8 \mathrm{~B}$ & 109.5 & $\mathrm{C} 45-\mathrm{C} 46-\mathrm{H} 46$ & 119.3 \\
\hline $\mathrm{H} 8 \mathrm{~A}-\mathrm{C} 8-\mathrm{H} 8 \mathrm{C}$ & 109.5 & $\mathrm{C} 45-\mathrm{C} 46-\mathrm{C} 47$ & $121.5(4)$ \\
\hline $\mathrm{H} 8 \mathrm{~B}-\mathrm{C} 8-\mathrm{H} 8 \mathrm{C}$ & 109.5 & $\mathrm{C} 47-\mathrm{C} 46-\mathrm{H} 46$ & 119.3 \\
\hline $\mathrm{C} 10-\mathrm{C} 9-\mathrm{P} 1$ & $118.8(3)$ & $\mathrm{C} 46-\mathrm{C} 47-\mathrm{C} 48$ & $117.4(3)$ \\
\hline $\mathrm{C} 10-\mathrm{C} 9-\mathrm{C} 14$ & $118.3(3)$ & C46-C47-C50 & $121.8(4)$ \\
\hline C14-C9-P1 & $121.9(3)$ & C48-C47-C50 & $120.8(4)$ \\
\hline $\mathrm{C} 9-\mathrm{C} 10-\mathrm{H} 10$ & 119.7 & $\mathrm{C} 47-\mathrm{C} 48-\mathrm{H} 48$ & 119.2 \\
\hline $\mathrm{C} 11-\mathrm{C} 10-\mathrm{C} 9$ & $120.6(3)$ & C49-C48-C47 & $121.7(4)$ \\
\hline $\mathrm{C} 11-\mathrm{C} 10-\mathrm{H} 10$ & 119.7 & $\mathrm{C} 49-\mathrm{C} 48-\mathrm{H} 48$ & 119.2 \\
\hline $\mathrm{C} 10-\mathrm{C} 11-\mathrm{H} 11$ & 119.4 & $\mathrm{C} 44-\mathrm{C} 49-\mathrm{H} 49$ & 119.7 \\
\hline $\mathrm{C} 10-\mathrm{C} 11-\mathrm{C} 12$ & $121.2(4)$ & C48-C49-C44 & $120.6(4)$ \\
\hline $\mathrm{C} 12-\mathrm{C} 11-\mathrm{H} 11$ & 119.4 & $\mathrm{C} 48-\mathrm{C} 49-\mathrm{H} 49$ & 119.7 \\
\hline C11-C12-C15 & $121.0(4)$ & C47-C50-H50A & 109.5 \\
\hline $\mathrm{C} 13-\mathrm{C} 12-\mathrm{C} 11$ & $117.8(3)$ & C47-C50-H50B & 109.5 \\
\hline C13-C12-C15 & $121.1(4)$ & C47-C50-H50C & 109.5 \\
\hline $\mathrm{C} 12-\mathrm{C} 13-\mathrm{H} 13$ & 119.2 & $\begin{array}{l}\mathrm{H} 50 \mathrm{~A}-\mathrm{C} 50- \\
\mathrm{H} 50 \mathrm{~B}\end{array}$ & 109.5 \\
\hline $\mathrm{C} 12-\mathrm{C} 13-\mathrm{C} 14$ & $121.5(4)$ & $\begin{array}{l}\mathrm{H} 50 \mathrm{~A}-\mathrm{C} 50- \\
\mathrm{H} 50 \mathrm{C}\end{array}$ & 109.5 \\
\hline $\mathrm{C} 14-\mathrm{C} 13-\mathrm{H} 13$ & 119.2 & $\mathrm{H} 50 \mathrm{~B}-\mathrm{C} 50-$ & 109.5 \\
\hline
\end{tabular}




\begin{tabular}{|l|l|l|l|}
\hline & & H50C & \\
\hline C9-C14-H14 & 119.8 & C52-C51-C57 & $120.0(4)$ \\
\hline C13-C14-C9 & $120.5(4)$ & C56-C51-C52 & $118.2(4)$ \\
\hline C13-C14-H14 & 119.8 & C56-C51-C57 & $121.8(4)$ \\
\hline C12-C15-H15A & 109.5 & C51-C52-H52 & 119.6 \\
\hline C12-C15-H15B & 109.5 & C51-C52-C53 & $120.8(4)$ \\
\hline C12-C15-H15C & 109.5 & C53-C52-H52 & 119.6 \\
\hline H15A-C15-H15B & 109.5 & C52-C53-H53 & 119.4 \\
\hline H15A-C15-H15C & 109.5 & C54-C53-C52 & $121.2(4)$ \\
\hline H15B-C15-H15C & 109.5 & C54-C53-H53 & 119.4 \\
\hline C17-C16-P1 & $123.6(3)$ & C53-C54-C55 & $117.3(4)$ \\
\hline C21-C16-P1 & $118.1(3)$ & C53-C54-C58 & $120.9(4)$ \\
\hline C21-C16-C17 & $118.2(3)$ & C55-C54-C58 & $121.8(4)$ \\
\hline C16-C17-H17 & 119.7 & C54-C55-H55 & 119.0 \\
\hline C18-C17-C16 & $120.6(3)$ & C56-C55-C54 & $121.9(4)$ \\
\hline C18-C17-H17 & 119.7 & C56-C55-H55 & 119.0 \\
\hline C17-C18-H18 & 119.4 & C51-C56-H56 & 119.7 \\
\hline C17-C18-C19 & $121.3(3)$ & C55-C56-C51 & $120.6(4)$ \\
\hline C19-C18-H18 & 119.4 & C55-C56-H56 & 119.7 \\
\hline C18-C19-C20 & $117.7(3)$ & C51-C57-H57A & 109.5 \\
\hline C18-C19-C22 & $122.0(3)$ & C51-C57-H57B & 109.5 \\
\hline C20-C19-C22 & $120.3(3)$ & C51-C57-H57C & 109.5 \\
\hline C19-C20-H20 & 119.5 & H57A-C57- & 109.5 \\
\hline C21-C20-C19 & $121.0(3)$ & H57B & 109.5 \\
\hline
\end{tabular}




\begin{tabular}{|c|c|c|c|}
\hline $\mathrm{C} 21-\mathrm{C} 20-\mathrm{H} 20$ & 119.5 & $\begin{array}{l}\text { H57B-C57- } \\
\text { H57C }\end{array}$ & 109.5 \\
\hline $\mathrm{C} 16-\mathrm{C} 21-\mathrm{H} 21$ & 119.4 & $\mathrm{C} 54-\mathrm{C} 58-\mathrm{H} 58 \mathrm{~A}$ & 109.5 \\
\hline $\mathrm{C} 20-\mathrm{C} 21-\mathrm{C} 16$ & $121.2(3)$ & C54-C58-H58B & 109.5 \\
\hline $\mathrm{C} 20-\mathrm{C} 21-\mathrm{H} 21$ & 119.4 & C54-C58-H58C & 109.5 \\
\hline $\mathrm{C} 19-\mathrm{C} 22-\mathrm{H} 22 \mathrm{~A}$ & 109.5 & $\begin{array}{l}\mathrm{H} 58 \mathrm{~A}-\mathrm{C} 58- \\
\mathrm{H} 58 \mathrm{~B}\end{array}$ & 109.5 \\
\hline C19-C22-H22B & 109.5 & $\begin{array}{l}\mathrm{H} 58 \mathrm{~A}-\mathrm{C} 58- \\
\mathrm{H} 58 \mathrm{C}\end{array}$ & 109.5 \\
\hline $\mathrm{C} 19-\mathrm{C} 22-\mathrm{H} 22 \mathrm{C}$ & 109.5 & $\begin{array}{l}\mathrm{H} 58 \mathrm{~B}-\mathrm{C} 58- \\
\mathrm{H} 58 \mathrm{C}\end{array}$ & 109.5 \\
\hline $\mathrm{H} 22 \mathrm{~A}-\mathrm{C} 22-\mathrm{H} 22 \mathrm{~B}$ & 109.5 & C60-C59-H59 & 119.1 \\
\hline $\mathrm{H} 22 \mathrm{~A}-\mathrm{C} 22-\mathrm{H} 22 \mathrm{C}$ & 109.5 & $\mathrm{C} 60-\mathrm{C} 59-\mathrm{C} 61^{\mathrm{i}}$ & $121.8(5)$ \\
\hline $\mathrm{H} 22 \mathrm{~B}-\mathrm{C} 22-\mathrm{H} 22 \mathrm{C}$ & 109.5 & $\mathrm{C} 61-\mathrm{C} 59-\mathrm{H} 59$ & 119.1 \\
\hline $\mathrm{C} 24-\mathrm{C} 23-\mathrm{P} 1$ & $119.0(3)$ & $\mathrm{C} 59-\mathrm{C} 60-\mathrm{H} 60$ & 119.1 \\
\hline $\mathrm{C} 28-\mathrm{C} 23-\mathrm{P} 1$ & $121.9(3)$ & C59-C60-C61 & $121.8(5)$ \\
\hline C28-C23-C24 & $119.1(3)$ & $\mathrm{C} 61-\mathrm{C} 60-\mathrm{H} 60$ & 119.1 \\
\hline $\mathrm{C} 23-\mathrm{C} 24-\mathrm{H} 24$ & 119.7 & C59i-C61-C62 & $122.6(6)$ \\
\hline C25-C24-C23 & $120.6(4)$ & C60-C61-C59i & $116.4(5)$ \\
\hline C25-C24-H24 & 119.7 & C60-C61-C62 & $121.0(5)$ \\
\hline C24-C25-H25 & 119.6 & $\mathrm{C} 61-\mathrm{C} 62-\mathrm{H} 62 \mathrm{~A}$ & 109.5 \\
\hline C24-C25-C26 & $120.9(3)$ & $\mathrm{C} 61-\mathrm{C} 62-\mathrm{H} 62 \mathrm{~B}$ & 109.5 \\
\hline C26-C25-H25 & 119.6 & $\mathrm{C} 61-\mathrm{C} 62-\mathrm{H} 62 \mathrm{C}$ & 109.5 \\
\hline C25-C26-C29 & $120.6(4)$ & $\begin{array}{l}\mathrm{H} 62 \mathrm{~A}-\mathrm{C} 62- \\
\mathrm{H} 62 \mathrm{~B}\end{array}$ & 109.5 \\
\hline C27-C26-C25 & $118.0(3)$ & $\begin{array}{l}\mathrm{H} 62 \mathrm{~A}-\mathrm{C} 62- \\
\mathrm{H} 62 \mathrm{C}\end{array}$ & 109.5 \\
\hline
\end{tabular}




\begin{tabular}{|c|c|c|c|}
\hline C27-C26-C29 & $121.3(4)$ & $\begin{array}{l}\mathrm{H} 62 \mathrm{~B}-\mathrm{C} 62- \\
\mathrm{H} 62 \mathrm{C}\end{array}$ & 109.5 \\
\hline $\mathrm{C} 26-\mathrm{C} 27-\mathrm{H} 27$ & 119.3 & & \\
\hline Os1-P1-C9-C10 & $-85.8(3)$ & $\begin{array}{l}\mathrm{C} 23-\mathrm{P} 1-\mathrm{C} 16- \\
\mathrm{C} 21\end{array}$ & $-93.0(3)$ \\
\hline Os1-P1-C9-C14 & $82.6(3)$ & $\begin{array}{l}\text { C23-C24-C25- } \\
\text { C26 }\end{array}$ & $0.0(6)$ \\
\hline $\begin{array}{l}\text { Os1-P1-C16- } \\
\text { C17 }\end{array}$ & $-146.1(3)$ & $\begin{array}{l}\text { C24-C23-C28- } \\
\text { C27 }\end{array}$ & $-0.9(6)$ \\
\hline $\begin{array}{l}\text { Os1-P1-C16- } \\
\text { C21 }\end{array}$ & $37.6(3)$ & $\begin{array}{l}\mathrm{C} 24-\mathrm{C} 25-\mathrm{C} 26- \\
\mathrm{C} 27\end{array}$ & $-0.1(6)$ \\
\hline $\begin{array}{l}\text { Os1-P1-C23- } \\
\mathrm{C} 24\end{array}$ & $174.7(2)$ & $\begin{array}{l}\text { C24-C25-C26- } \\
\text { C29 }\end{array}$ & $-179.8(4)$ \\
\hline $\begin{array}{l}\text { Os1-P1-C23- } \\
\text { C28 }\end{array}$ & $-2.4(4)$ & $\begin{array}{l}\text { C25-C26-C27- } \\
\text { C28 }\end{array}$ & $-0.3(6)$ \\
\hline Os1-O5-C5-O6 & $12.5(5)$ & $\begin{array}{l}\text { C26-C27-C28- } \\
\text { C23 }\end{array}$ & $0.8(6)$ \\
\hline Os1-O5-C5-C6 & $-166.5(2)$ & $\begin{array}{l}\text { C28-C23-C24- } \\
\text { C25 }\end{array}$ & $0.5(6)$ \\
\hline Os1-O7-C7-O8 & $10.6(5)$ & $\begin{array}{l}\mathrm{C} 29-\mathrm{C} 26-\mathrm{C} 27- \\
\mathrm{C} 28\end{array}$ & $179.4(4)$ \\
\hline Os1-O7-C7-C8 & $-169.2(3)$ & $\begin{array}{l}\text { C30-P2-C37- } \\
\text { C38 }\end{array}$ & $77.4(3)$ \\
\hline $\begin{array}{l}\text { Os2-P2-C30- } \\
\text { C31 }\end{array}$ & $-61.2(3)$ & $\begin{array}{l}\text { C30-P2-C37- } \\
\text { C42 }\end{array}$ & $-106.2(3)$ \\
\hline $\begin{array}{l}\text { Os2-P2-C30- } \\
\text { C35 }\end{array}$ & $116.4(3)$ & $\begin{array}{l}\text { C30-P2-C44- } \\
\text { C45 }\end{array}$ & $5.9(3)$ \\
\hline Os2-P2-C37- & $-50.0(3)$ & C30-P2-C44- & $-171.6(3)$ \\
\hline
\end{tabular}




\begin{tabular}{|c|c|c|c|}
\hline C38 & & C49 & \\
\hline $\begin{array}{l}\text { Os2-P2-C37- } \\
\text { C42 }\end{array}$ & $126.4(3)$ & $\begin{array}{l}\text { C30-C31-C32- } \\
\text { C33 }\end{array}$ & $-0.8(5)$ \\
\hline $\begin{array}{l}\text { Os2-P2-C44- } \\
\text { C45 }\end{array}$ & $131.9(3)$ & $\begin{array}{l}\mathrm{C} 31-\mathrm{C} 30-\mathrm{C} 35- \\
\mathrm{C} 34\end{array}$ & $0.0(5)$ \\
\hline $\begin{array}{l}\text { Os2-P2-C44- } \\
\text { C49 }\end{array}$ & $-45.6(3)$ & $\begin{array}{l}\text { C31-C32-C33- } \\
\text { C34 }\end{array}$ & $0.2(5)$ \\
\hline Os2-O6-C5-O5 & $10.4(5)$ & $\begin{array}{l}\text { C31-C32-C33- } \\
\text { C36 }\end{array}$ & $-178.7(3)$ \\
\hline Os2-O6-C5-C6 & $-170.6(3)$ & $\begin{array}{l}\text { C32-C33-C34- } \\
\text { C35 }\end{array}$ & $0.5(5)$ \\
\hline Os2-O8-C7-O7 & $13.3(6)$ & $\begin{array}{l}\text { C33-C34-C35- } \\
\text { C30 }\end{array}$ & $-0.6(6)$ \\
\hline Os2-O8-C7-C8 & $-166.9(3)$ & $\begin{array}{l}\text { C35-C30-C31- } \\
\text { C32 }\end{array}$ & $0.6(5)$ \\
\hline $\mathrm{P} 1-\mathrm{C} 9-\mathrm{C} 10-\mathrm{C} 11$ & $169.3(3)$ & $\begin{array}{l}\text { C36-C33-C34- } \\
\text { C35 }\end{array}$ & $179.4(4)$ \\
\hline $\mathrm{P} 1-\mathrm{C} 9-\mathrm{C} 14-\mathrm{C} 13$ & $-169.5(3)$ & $\begin{array}{l}\text { C37-P2-C30- } \\
\text { C31 }\end{array}$ & $172.9(3)$ \\
\hline $\begin{array}{l}\mathrm{P} 1-\mathrm{C} 16-\mathrm{C} 17- \\
\mathrm{C} 18\end{array}$ & $-177.6(3)$ & $\begin{array}{l}\text { C37-P2-C30- } \\
\text { C35 }\end{array}$ & $-9.5(3)$ \\
\hline $\begin{array}{l}\mathrm{P} 1-\mathrm{C} 16-\mathrm{C} 21- \\
\mathrm{C} 20\end{array}$ & $177.5(3)$ & $\begin{array}{l}\text { C37-P2-C44- } \\
\text { C45 }\end{array}$ & $-104.4(3)$ \\
\hline $\begin{array}{l}\mathrm{P} 1-\mathrm{C} 23-\mathrm{C} 24- \\
\mathrm{C} 25\end{array}$ & $-176.8(3)$ & $\begin{array}{l}\text { C37-P2-C44- } \\
\text { C49 }\end{array}$ & $78.1(3)$ \\
\hline $\begin{array}{l}\mathrm{P} 1-\mathrm{C} 23-\mathrm{C} 28- \\
\mathrm{C} 27\end{array}$ & $176.3(3)$ & $\begin{array}{l}\text { C37-C38-C39- } \\
\text { C40 }\end{array}$ & $-1.2(6)$ \\
\hline $\begin{array}{l}\text { P2-C30-C31- } \\
\text { C32 }\end{array}$ & $178.4(3)$ & $\begin{array}{l}\text { C38-C37-C42- } \\
\text { C41 }\end{array}$ & $2.9(6)$ \\
\hline
\end{tabular}




\begin{tabular}{|c|c|c|c|}
\hline $\begin{array}{l}\text { P2-C30-C35- } \\
\text { C34 }\end{array}$ & $-177.6(3)$ & $\begin{array}{l}\text { C38-C39-C40- } \\
\text { C41 }\end{array}$ & $2.8(6)$ \\
\hline $\begin{array}{l}\text { P2-C37-C38- } \\
\text { C39 }\end{array}$ & $174.9(3)$ & $\begin{array}{l}\text { C38-C39-C40- } \\
\text { C43 }\end{array}$ & $-175.8(4)$ \\
\hline $\begin{array}{l}\mathrm{P} 2-\mathrm{C} 37-\mathrm{C} 42- \\
\mathrm{C} 41\end{array}$ & $-173.5(3)$ & $\begin{array}{l}\text { C39-C40-C41- } \\
\text { C42 }\end{array}$ & $-1.6(6)$ \\
\hline $\begin{array}{l}\text { P2-C44-C45- } \\
\text { C46 }\end{array}$ & $-176.9(3)$ & $\begin{array}{l}\text { C40-C41-C42- } \\
\text { C37 }\end{array}$ & $-1.3(6)$ \\
\hline $\begin{array}{l}\mathrm{P} 2-\mathrm{C} 44-\mathrm{C} 49- \\
\mathrm{C} 48\end{array}$ & $177.2(3)$ & $\begin{array}{l}\text { C42-C37-C38- } \\
\text { C39 }\end{array}$ & $-1.7(6)$ \\
\hline $\mathrm{C} 9-\mathrm{P} 1-\mathrm{C} 16-\mathrm{C} 17$ & $-23.4(4)$ & $\begin{array}{l}\mathrm{C} 43-\mathrm{C} 40-\mathrm{C} 41- \\
\mathrm{C} 42\end{array}$ & $177.1(4)$ \\
\hline $\mathrm{C} 9-\mathrm{P} 1-\mathrm{C} 16-\mathrm{C} 21$ & $160.3(3)$ & $\begin{array}{l}\text { C44-P2-C30- } \\
\text { C31 }\end{array}$ & $63.7(3)$ \\
\hline $\mathrm{C} 9-\mathrm{P} 1-\mathrm{C} 23-\mathrm{C} 24$ & $54.1(3)$ & $\begin{array}{l}\mathrm{C} 44-\mathrm{P} 2-\mathrm{C} 30- \\
\mathrm{C} 35\end{array}$ & $-118.7(3)$ \\
\hline $\mathrm{C} 9-\mathrm{P} 1-\mathrm{C} 23-\mathrm{C} 28$ & $-123.0(3)$ & $\begin{array}{l}\text { C44-P2-C37- } \\
\text { C38 }\end{array}$ & $-174.2(3)$ \\
\hline $\begin{array}{l}\mathrm{C} 9-\mathrm{C} 10-\mathrm{C} 11- \\
\mathrm{C} 12\end{array}$ & $0.5(5)$ & $\begin{array}{l}\mathrm{C} 44-\mathrm{P} 2-\mathrm{C} 37- \\
\mathrm{C} 42\end{array}$ & $2.2(4)$ \\
\hline $\begin{array}{l}\mathrm{C} 10-\mathrm{C} 9-\mathrm{C} 14- \\
\mathrm{C} 13\end{array}$ & $-1.0(5)$ & $\begin{array}{l}\mathrm{C} 44-\mathrm{C} 45-\mathrm{C} 46- \\
\mathrm{C} 47\end{array}$ & $0.1(6)$ \\
\hline $\begin{array}{l}\mathrm{C} 10-\mathrm{C} 11-\mathrm{C} 12- \\
\mathrm{C} 13\end{array}$ & $-0.8(5)$ & $\begin{array}{l}\mathrm{C} 45-\mathrm{C} 44-\mathrm{C} 49- \\
\mathrm{C} 48\end{array}$ & $-0.4(5)$ \\
\hline $\begin{array}{l}\mathrm{C} 10-\mathrm{C} 11-\mathrm{C} 12- \\
\mathrm{C} 15\end{array}$ & $-178.8(3)$ & $\begin{array}{l}\mathrm{C} 45-\mathrm{C} 46-\mathrm{C} 47- \\
\mathrm{C} 48\end{array}$ & $-1.2(5)$ \\
\hline $\begin{array}{l}\text { C11-C12-C13- } \\
\text { C14 }\end{array}$ & $0.1(6)$ & $\begin{array}{l}\mathrm{C} 45-\mathrm{C} 46-\mathrm{C} 47- \\
\mathrm{C} 50\end{array}$ & $177.9(4)$ \\
\hline $\mathrm{C} 12-\mathrm{C} 13-\mathrm{C} 14-$ & $0.8(6)$ & C46-C47-C48- & $1.4(6)$ \\
\hline
\end{tabular}




\begin{tabular}{|c|c|c|c|}
\hline C9 & & C49 & \\
\hline $\begin{array}{l}\mathrm{C} 14-\mathrm{C} 9-\mathrm{C} 10- \\
\mathrm{C} 11\end{array}$ & $0.4(5)$ & $\begin{array}{l}\text { C47-C48-C49- } \\
\text { C44 }\end{array}$ & $-0.6(6)$ \\
\hline $\begin{array}{l}\mathrm{C} 15-\mathrm{C} 12-\mathrm{C} 13- \\
\mathrm{C} 14\end{array}$ & $178.2(4)$ & $\begin{array}{l}\mathrm{C} 49-\mathrm{C} 44-\mathrm{C} 45- \\
\mathrm{C} 46\end{array}$ & $0.7(5)$ \\
\hline $\mathrm{C} 16-\mathrm{P} 1-\mathrm{C} 9-\mathrm{C} 10$ & $148.4(3)$ & $\begin{array}{l}\text { C50-C47-C48- } \\
\text { C49 }\end{array}$ & $-177.7(4)$ \\
\hline $\mathrm{C} 16-\mathrm{P} 1-\mathrm{C} 9-\mathrm{C} 14$ & $-43.1(3)$ & $\begin{array}{l}\text { C51-C52-C53- } \\
\text { C54 }\end{array}$ & $-0.9(6)$ \\
\hline $\begin{array}{l}\mathrm{C} 16-\mathrm{P} 1-\mathrm{C} 23- \\
\mathrm{C} 24\end{array}$ & $-57.0(3)$ & $\begin{array}{l}\text { C52-C51-C56- } \\
\text { C55 }\end{array}$ & $-0.9(6)$ \\
\hline $\begin{array}{l}\mathrm{C} 16-\mathrm{P} 1-\mathrm{C} 23- \\
\mathrm{C} 28\end{array}$ & $125.8(3)$ & $\begin{array}{l}\text { C52-C53-C54- } \\
\text { C55 }\end{array}$ & $0.3(6)$ \\
\hline $\begin{array}{l}\mathrm{C} 16-\mathrm{C} 17-\mathrm{C} 18- \\
\mathrm{C} 19\end{array}$ & $0.4(6)$ & $\begin{array}{l}\mathrm{C} 52-\mathrm{C} 53-\mathrm{C} 54- \\
\mathrm{C} 58\end{array}$ & $178.2(4)$ \\
\hline $\begin{array}{l}\mathrm{C} 17-\mathrm{C} 16-\mathrm{C} 21- \\
\mathrm{C} 20\end{array}$ & $1.0(6)$ & $\begin{array}{l}\text { C53-C54-C55- } \\
\text { C56 }\end{array}$ & $-0.1(6)$ \\
\hline $\begin{array}{l}\text { C17-C18-C19- } \\
\text { C20 }\end{array}$ & $0.7(6)$ & $\begin{array}{l}\text { C54-C55-C56- } \\
\text { C51 }\end{array}$ & $0.3(6)$ \\
\hline $\begin{array}{l}\text { C17-C18-C19- } \\
\text { C22 }\end{array}$ & $-177.6(4)$ & $\begin{array}{l}\text { C56-C51-C52- } \\
\text { C53 }\end{array}$ & $1.2(6)$ \\
\hline $\begin{array}{l}\text { C18-C19-C20- } \\
\text { C21 }\end{array}$ & $-0.9(6)$ & $\begin{array}{l}\text { C57-C51-C52- } \\
\text { C53 }\end{array}$ & $-178.5(4)$ \\
\hline $\begin{array}{l}\mathrm{C} 19-\mathrm{C} 20-\mathrm{C} 21- \\
\mathrm{C} 16\end{array}$ & $0.1(6)$ & $\begin{array}{l}\text { C57-C51-C56- } \\
\text { C55 }\end{array}$ & $178.8(4)$ \\
\hline $\begin{array}{l}\mathrm{C} 21-\mathrm{C} 16-\mathrm{C} 17- \\
\mathrm{C} 18\end{array}$ & $-1.2(6)$ & $\begin{array}{l}\text { C58-C54-C55- } \\
\text { C56 }\end{array}$ & $-177.9(4)$ \\
\hline $\begin{array}{l}\mathrm{C} 22-\mathrm{C} 19-\mathrm{C} 20- \\
\mathrm{C} 21\end{array}$ & $177.3(3)$ & $\begin{array}{l}\text { C59-C60-C61- } \\
\text { C59i }\end{array}$ & $-0.4(8)$ \\
\hline
\end{tabular}




\begin{tabular}{|l|l|l|l|}
\hline C23-P1-C9-C10 & $40.8(3)$ & $\begin{array}{l}\text { C59-C60-C61- } \\
\text { C62 }\end{array}$ & $-179.2(5)$ \\
\hline C23-P1-C9-C14 & $-150.7(3)$ & $\begin{array}{l}\text { C61-C59-C60- } \\
\text { C61 }\end{array}$ & $0.4(8)$ \\
\hline $\begin{array}{l}\text { C23-P1-C16- } \\
\text { C17 }\end{array}$ & $83.3(3)$ & & \\
\hline
\end{tabular}

Symmetry code: (i) $-x+1,-y,-z+1$.

(5)

Crystal data

\begin{tabular}{|l|l|}
\hline $\mathrm{C}_{12} \mathrm{H}_{10} \mathrm{O}_{10} \mathrm{Os}_{2}$ & $D_{\mathrm{x}}=2.792 \mathrm{Mg} \mathrm{m}^{-3}$ \\
\hline$M_{r}=694.60$ & Cu Ka radiation, $\lambda=1.54184 \AA$ \\
\hline Orthorhombic, Pbca & Cell parameters from 11012 reflections \\
\hline$a=9.79942(10) \AA$ & $\theta=5.7-73.4^{\circ}$ \\
\hline$b=15.51827(19) \AA$ & $\mu=29.08 \mathrm{~mm}^{-1}$ \\
\hline$c=21.7339(2) \AA$ & $T=100 \mathrm{~K}$ \\
\hline$V=3305.08(6) \AA^{3}$ & Trapezoid, colorless \\
\hline$Z=8$ & $0.08 \times 0.06 \times 0.04 \mathrm{~mm}$ \\
\hline$F(000)=2512$ & \\
\hline
\end{tabular}

Data collection

\begin{tabular}{|l|l|}
\hline $\begin{array}{l}\text { Rigaku SuperNova AtlasS2 CCD } \\
\text { diffractometer }\end{array}$ & 3300 independent reflections \\
\hline $\begin{array}{l}\text { Radiation source: micro-focus sealed X-ray } \\
\text { tube, SuperNova }(\mathrm{Cu}) \text { X-ray Source }\end{array}$ & 3196 reflections with $I>2 \sigma(I)$ \\
\hline Mirror monochromator & $R_{\mathrm{int}}=0.027$ \\
\hline Detector resolution: 5.2387 pixels $\mathrm{mm}^{-1}$ & $\theta_{\max }=73.7^{\circ}, \theta_{\min }=4.1^{\circ}$ \\
\hline
\end{tabular}




\begin{tabular}{|l|l|}
\hline$\omega$ scans & $h=-11 \rightarrow 12$ \\
\hline $\begin{array}{c}\text { Absorption correction: multi-scan } \\
\text { CrysAlis } P R O \text { (Rigaku OD, 2015) }\end{array}$ & $k=-18 \rightarrow 19$ \\
\hline$T_{\min }=0.637, T_{\max }=1.000$ & $I=-27 \rightarrow 26$ \\
\hline 16571 measured reflections & \\
\hline
\end{tabular}

\section{Refinement}

\begin{tabular}{|c|c|}
\hline Refinement on $F^{2}$ & 0 restraints \\
\hline Least-squares matrix: full & $\begin{array}{l}\text { Hydrogen site location: inferred from } \\
\text { neighbouring sites }\end{array}$ \\
\hline$R\left[F^{2}>2 \mathrm{~s}\left(F^{2}\right)\right]=0.018$ & H-atom parameters constrained \\
\hline$w R\left(F^{2}\right)=0.039$ & $\begin{array}{l}w=1 /\left[\sigma^{2}\left(F_{0}^{2}\right)+(0.0142 P)^{2}+12 . P\right] \\
\text { where } P=\left(F_{0}^{2}+2 F_{c}^{2}\right) / 3\end{array}$ \\
\hline$S=1.03$ & $(\Delta / \sigma)_{\max }=0.003$ \\
\hline 3300 reflections & $\Delta\rangle_{\max }=0.55{\mathrm{e} \AA^{-3}}^{-3}$ \\
\hline 219 parameters & $\Delta\rangle_{\min }=-0.93$ e $\AA^{-3}$ \\
\hline
\end{tabular}

\section{Special details}

Geometry. All esds (except the esd in the dihedral angle between two l.s. planes) are estimated using the full covariance matrix. The cell esds are taken into account individually in the estimation of esds in distances, angles and torsion angles; correlations between esds in cell parameters are only used when they are defined by crystal symmetry. An approximate (isotropic) treatment of cell esds is used for estimating esds involving l.s. planes.

Fractional atomic coordinates and isotropic or equivalent isotropic displacement parameters $\left(\AA^{2}\right)$ for (5)

\begin{tabular}{|l|l|l|l|l|}
\hline$x$ & $y$ & $z$ & $U_{\text {iso }}^{*} / U_{\text {eq }}$ \\
\hline
\end{tabular}




\begin{tabular}{|c|c|c|c|c|}
\hline Os1 & $0.80792(2)$ & $0.55113(2)$ & $0.59651(2)$ & $0.00927(5)$ \\
\hline Os2 & $0.58526(2)$ & $0.65884(2)$ & $0.58989(2)$ & 0.00867 (5) \\
\hline 01 & 0.7665 (3) & $0.47459(19)$ & $0.46970(12)$ & $0.0240(6)$ \\
\hline $\mathrm{O} 2$ & $1.0011(3)$ & $0.68133(19)$ & $0.54088(14)$ & $0.0258(6)$ \\
\hline O3 & $1.0342(3)$ & $0.41963(19)$ & $0.63032(15)$ & $0.0298(7)$ \\
\hline $\mathrm{O} 4$ & $0.5287(3)$ & $0.60269(19)$ & $0.45938(12)$ & $0.0228(6)$ \\
\hline O5 & 0.7527 (3) & $0.80956(17)$ & $0.54447(13)$ & $0.0204(6)$ \\
\hline O6 & 0.3175 (3) & $0.76514(18)$ & $0.58797(12)$ & $0.0198(6)$ \\
\hline $\mathrm{O} 7$ & $0.4843(2)$ & $0.55431(16)$ & $0.63274(12)$ & $0.0141(5)$ \\
\hline $\mathrm{O} 8$ & $0.6683(2)$ & $0.46936(16)$ & $0.64223(11)$ & $0.0133(5)$ \\
\hline O9 & $0.6379(2)$ & $0.68838(16)$ & $0.68238(11)$ & $0.0139(5)$ \\
\hline O10 & $0.8253(2)$ & $0.60506(16)$ & $0.68545(11)$ & $0.0125(5)$ \\
\hline C1 & $0.7804(3)$ & $0.5025(2)$ & $0.51766(17)$ & $0.0163(7)$ \\
\hline C2 & $0.9285(4)$ & $0.6318(2)$ & $0.56154(17)$ & $0.0169(7)$ \\
\hline C3 & 0.9519 (4) & $0.4673(2)$ & $0.61907(17)$ & $0.0167(7)$ \\
\hline C4 & $0.5482(3)$ & $0.6241(2)$ & $0.50883(17)$ & $0.0139(7)$ \\
\hline C5 & $0.6878(3)$ & $0.7526(2)$ & $0.56034(16)$ & $0.0136(7)$ \\
\hline C6 & 0.4139 (3) & $0.7261(2)$ & $0.59352(15)$ & $0.0133(7)$ \\
\hline C7 & $0.5444(3)$ & $0.4876(2)$ & $0.65198(15)$ & $0.0128(7)$ \\
\hline C8 & $0.4621(4)$ & $0.4258(2)$ & $0.69069(17)$ & $0.0179(7)$ \\
\hline H8A & 0.382532 & 0.407957 & 0.667429 & $0.021^{*}$ \\
\hline H8B & 0.430190 & 0.455912 & 0.727057 & $0.021^{*}$ \\
\hline C9 & $0.5398(5)$ & $0.3462(3)$ & $0.7107(2)$ & $0.0339(11)$ \\
\hline H9A & 0.563801 & 0.312751 & 0.675178 & $0.051^{*}$ \\
\hline H9B & 0.483504 & 0.312306 & 0.737563 & $0.051^{*}$ \\
\hline
\end{tabular}




\begin{tabular}{|l|l|l|l|l|}
\hline H9C & 0.621204 & 0.363151 & 0.732080 & $0.051^{*}$ \\
\hline C10 & $0.7394(3)$ & $0.6553(2)$ & $0.70948(15)$ & $0.0112(6)$ \\
\hline C11 & $0.7578(3)$ & $0.6738(2)$ & $0.77686(16)$ & $0.0141(7)$ \\
\hline H11A & 0.726967 & 0.731813 & 0.786111 & $0.017^{\star}$ \\
\hline H11B & 0.853480 & 0.669456 & 0.787837 & $0.017^{\star}$ \\
\hline C12 & $0.6742(4)$ & $0.6082(3)$ & $0.81360(17)$ & $0.0205(8)$ \\
\hline H12A & 0.703193 & 0.550999 & 0.803112 & $0.031^{\star}$ \\
\hline H12B & 0.579226 & 0.614842 & 0.803915 & $0.031^{\star}$ \\
\hline H12C & 0.687896 & 0.617754 & 0.856809 & $0.031^{\star}$ \\
\hline
\end{tabular}

Atomic displacement parameters $\left(\AA^{2}\right)$ for (5)

\begin{tabular}{|l|l|l|l|l|l|l|}
\hline & $U^{11}$ & $U^{22}$ & $U^{\beta 3}$ & $U^{12}$ & $U^{13}$ & $U^{23}$ \\
\hline Os1 & $\begin{array}{l}0.00868 \\
(7)\end{array}$ & $\begin{array}{l}0.00912 \\
(8)\end{array}$ & $\begin{array}{l}0.01001 \\
(8)\end{array}$ & $\begin{array}{l}0.00042 \\
(5)\end{array}$ & $\begin{array}{l}0.00130 \\
(5)\end{array}$ & $\begin{array}{l}0.00014 \\
(5)\end{array}$ \\
\hline Os2 & $\begin{array}{l}0.00900 \\
(7)\end{array}$ & $\begin{array}{l}0.00854 \\
(8)\end{array}$ & $\begin{array}{l}0.00847 \\
(8)\end{array}$ & $\begin{array}{l}0.00035 \\
(5)\end{array}$ & $\begin{array}{l}-0.00063 \\
(5)\end{array}$ & $\begin{array}{l}0.00031 \\
(5)\end{array}$ \\
\hline O1 & 0.0343 & 0.0243 & 0.0134 & 0.0077 & -0.0004 & -0.0050 \\
& $(15)$ & $(15)$ & $(13)$ & $(12)$ & $(12)$ & $(11)$ \\
\hline O2 & 0.0169 & 0.0243 & 0.0362 & -0.0014 & 0.0072 & 0.0124 \\
& $(13)$ & $(15)$ & $(17)$ & $(11)$ & $(12)$ & $(13)$ \\
\hline O3 & 0.0264 & 0.0216 & 0.0415 & 0.0127 & -0.0082 & 0.0003 \\
& $(15)$ & $(15)$ & $(18)$ & $(13)$ & $(13)$ & $(13)$ \\
\hline O4 & 0.0245 & 0.0281 & 0.0158 & 0.0045 & -0.0081 & -0.0098 \\
& $(13)$ & $(15)$ & $(13)$ & $(12)$ & $(11)$ & $(11)$ \\
\hline O5 & 0.0189 & 0.0149 & 0.0275 & -0.0041 & 0.0005 & 0.0054 \\
& $(12)$ & $(13)$ & $(14)$ & $(11)$ & $(11)$ & $(11)$ \\
\hline O6 & 0.0147 & 0.0227 & 0.0219 & 0.0063 & -0.0020 & -0.0004 \\
\hline
\end{tabular}




\begin{tabular}{|c|c|c|c|c|c|c|}
\hline & (12) & (14) & (14) & (11) & (10) & (11) \\
\hline $\mathrm{O} 7$ & $\begin{array}{l}0.0119 \\
(11)\end{array}$ & $\begin{array}{l}0.0116 \\
(12)\end{array}$ & $\begin{array}{l}0.0188 \\
(12)\end{array}$ & $\begin{array}{l}-0.0013 \\
(9)\end{array}$ & $\begin{array}{l}0.0019 \\
(10)\end{array}$ & $\begin{array}{l}0.0043 \\
(10)\end{array}$ \\
\hline O8 & $\begin{array}{l}0.0111 \\
(11)\end{array}$ & $\begin{array}{l}0.0115 \\
(12)\end{array}$ & $\begin{array}{l}0.0174 \\
(12)\end{array}$ & $0.0000(9)$ & $0.0012(9)$ & $\begin{array}{l}0.0033 \\
(10)\end{array}$ \\
\hline O9 & $\begin{array}{l}0.0153 \\
(11)\end{array}$ & $\begin{array}{l}0.0159 \\
(12)\end{array}$ & $\begin{array}{l}0.0104 \\
(11)\end{array}$ & $\begin{array}{l}0.0040 \\
(10)\end{array}$ & $\begin{array}{l}-0.0030 \\
(9)\end{array}$ & $\begin{array}{l}-0.0018 \\
(9)\end{array}$ \\
\hline O10 & $\begin{array}{l}0.0109 \\
(11)\end{array}$ & $\begin{array}{l}0.0163 \\
(12)\end{array}$ & $\begin{array}{l}0.0103 \\
(11)\end{array}$ & $0.0018(9)$ & $\begin{array}{l}-0.0020 \\
(9)\end{array}$ & $\begin{array}{l}-0.0021 \\
(9)\end{array}$ \\
\hline C1 & $\begin{array}{l}0.0144 \\
(16)\end{array}$ & $\begin{array}{l}0.0137 \\
(17)\end{array}$ & $\begin{array}{l}0.0208 \\
(19)\end{array}$ & $\begin{array}{l}0.0033 \\
(13)\end{array}$ & $\begin{array}{l}0.0034 \\
(14)\end{array}$ & $\begin{array}{l}0.0014 \\
(15)\end{array}$ \\
\hline C2 & $\begin{array}{l}0.0150 \\
(17)\end{array}$ & $\begin{array}{l}0.0150 \\
(17)\end{array}$ & $\begin{array}{l}0.0206 \\
(18)\end{array}$ & $\begin{array}{l}0.0061 \\
(14)\end{array}$ & $\begin{array}{l}-0.0001 \\
(14)\end{array}$ & $\begin{array}{l}0.0008 \\
(15)\end{array}$ \\
\hline C3 & $\begin{array}{l}0.0166 \\
(17)\end{array}$ & $\begin{array}{l}0.0160 \\
(18)\end{array}$ & $\begin{array}{l}0.0175 \\
(17)\end{array}$ & $\begin{array}{l}-0.0020 \\
(15)\end{array}$ & $\begin{array}{l}0.0024 \\
(14)\end{array}$ & $\begin{array}{l}-0.0001 \\
(14)\end{array}$ \\
\hline C4 & $\begin{array}{l}0.0096 \\
(15)\end{array}$ & $\begin{array}{l}0.0128 \\
(16)\end{array}$ & $\begin{array}{l}0.0195 \\
(18)\end{array}$ & $\begin{array}{l}0.0012 \\
(13)\end{array}$ & $\begin{array}{l}-0.0005 \\
(13)\end{array}$ & $\begin{array}{l}0.0017 \\
(14)\end{array}$ \\
\hline C5 & $\begin{array}{l}0.0146 \\
(16)\end{array}$ & $\begin{array}{l}0.0135 \\
(17)\end{array}$ & $\begin{array}{l}0.0128 \\
(16)\end{array}$ & $\begin{array}{l}0.0050 \\
(14)\end{array}$ & $\begin{array}{l}-0.0038 \\
(13)\end{array}$ & $\begin{array}{l}0.0003 \\
(13)\end{array}$ \\
\hline C6 & $\begin{array}{l}0.0147 \\
(17)\end{array}$ & $\begin{array}{l}0.0143 \\
(17)\end{array}$ & $\begin{array}{l}0.0109 \\
(16)\end{array}$ & $\begin{array}{l}-0.0035 \\
(14)\end{array}$ & $\begin{array}{l}0.0028 \\
(13)\end{array}$ & $\begin{array}{l}0.0004 \\
(13)\end{array}$ \\
\hline $\mathrm{C7}$ & $\begin{array}{l}0.0138 \\
(16)\end{array}$ & $\begin{array}{l}0.0131 \\
(16)\end{array}$ & $\begin{array}{l}0.0116 \\
(15)\end{array}$ & $\begin{array}{l}-0.0049 \\
(13)\end{array}$ & $\begin{array}{l}-0.0012 \\
(13)\end{array}$ & $\begin{array}{l}-0.0024 \\
(13)\end{array}$ \\
\hline C8 & $\begin{array}{l}0.0165 \\
(17)\end{array}$ & $\begin{array}{l}0.0178 \\
(18)\end{array}$ & $\begin{array}{l}0.0193 \\
(18)\end{array}$ & $\begin{array}{l}-0.0037 \\
(14)\end{array}$ & $\begin{array}{l}0.0012 \\
(14)\end{array}$ & $\begin{array}{l}0.0058 \\
(15)\end{array}$ \\
\hline C9 & $0.026(2)$ & $0.023(2)$ & $0.053(3)$ & $\begin{array}{l}-0.0025 \\
(17)\end{array}$ & $0.009(2)$ & $0.022(2)$ \\
\hline C10 & $\begin{array}{l}0.0102 \\
(14)\end{array}$ & $\begin{array}{l}0.0118 \\
(16)\end{array}$ & $\begin{array}{l}0.0115 \\
(15)\end{array}$ & $\begin{array}{l}-0.0040 \\
(12)\end{array}$ & $\begin{array}{l}-0.0004 \\
(13)\end{array}$ & $\begin{array}{l}0.0019 \\
(13)\end{array}$ \\
\hline
\end{tabular}




\begin{tabular}{|l|l|l|l|l|l|l|}
\hline C11 & $\begin{array}{l}0.0132 \\
(15)\end{array}$ & $\begin{array}{l}0.0175 \\
(17)\end{array}$ & $\begin{array}{l}0.0115 \\
(16)\end{array}$ & $\begin{array}{l}0.0006 \\
(14)\end{array}$ & -0.0019 & -0.0009 \\
$(13)$ & $(14)$ \\
\hline C12 & $\begin{array}{l}0.0131 \\
(16)\end{array}$ & $0.034(2)$ & $\begin{array}{l}0.0141 \\
(17)\end{array}$ & $\begin{array}{l}-0.0032 \\
(15)\end{array}$ & $\begin{array}{l}0.0002 \\
(14)\end{array}$ & $\begin{array}{l}0.0042 \\
(16)\end{array}$ \\
\hline
\end{tabular}

Geometric parameters (Å, $\stackrel{\circ}{)}$ for (5)

\begin{tabular}{|c|c|c|c|}
\hline Os1-Os2 & $2.7523(2)$ & $\mathrm{O} 8-\mathrm{C} 7$ & $1.265(4)$ \\
\hline Os1-O8 & $2.114(2)$ & $\mathrm{O} 9-\mathrm{C} 10$ & $1.265(4)$ \\
\hline Os1-O10 & $2.113(2)$ & $\mathrm{O} 10-\mathrm{C} 10$ & $1.261(4)$ \\
\hline Os1-C1 & $1.891(4)$ & C7-C8 & $1.509(5)$ \\
\hline Os1-C2 & $1.882(4)$ & $\mathrm{C} 8-\mathrm{H} 8 \mathrm{~A}$ & 0.9700 \\
\hline Os1-C3 & $1.981(4)$ & $\mathrm{C} 8-\mathrm{H} 8 \mathrm{~B}$ & 0.9700 \\
\hline Os2-O7 & $2.116(2)$ & $\mathrm{C} 8-\mathrm{C} 9$ & $1.515(5)$ \\
\hline Os2-09 & 2.125 (2) & $\mathrm{C} 9-\mathrm{H} 9 \mathrm{~A}$ & 0.9600 \\
\hline Os2-C4 & $1.878(4)$ & $\mathrm{C} 9-\mathrm{H} 9 \mathrm{~B}$ & 0.9600 \\
\hline Os2-C5 & $1.882(4)$ & $\mathrm{C} 9-\mathrm{H} 9 \mathrm{C}$ & 0.9600 \\
\hline Os2-C6 & $1.979(4)$ & $\mathrm{C} 10-\mathrm{C} 11$ & $1.503(5)$ \\
\hline $\mathrm{O} 1-\mathrm{C} 1$ & $1.137(5)$ & $\mathrm{C} 11-\mathrm{H} 11 \mathrm{~A}$ & 0.9700 \\
\hline $\mathrm{O} 2-\mathrm{C} 2$ & $1.139(5)$ & $\mathrm{C} 11-\mathrm{H} 11 \mathrm{~B}$ & 0.9700 \\
\hline $\mathrm{O} 3-\mathrm{C} 3$ & $1.122(5)$ & $\mathrm{C} 11-\mathrm{C} 12$ & $1.531(5)$ \\
\hline O4-C4 & $1.141(5)$ & $\mathrm{C} 12-\mathrm{H} 12 \mathrm{~A}$ & 0.9600 \\
\hline O5-C5 & $1.142(5)$ & $\mathrm{C} 12-\mathrm{H} 12 \mathrm{~B}$ & 0.9600 \\
\hline $\mathrm{O} 6-\mathrm{C} 6$ & $1.128(4)$ & $\mathrm{C} 12-\mathrm{H} 12 \mathrm{C}$ & 0.9600 \\
\hline $\mathrm{O} 7-\mathrm{C} 7$ & $1.262(4)$ & & \\
\hline O8-Os1-Os2 & $82.89(6)$ & $\mathrm{O} 2-\mathrm{C} 2-\mathrm{Os} 1$ & $179.2(4)$ \\
\hline
\end{tabular}




\begin{tabular}{|c|c|c|c|}
\hline O10-Os1-Os2 & $82.61(6)$ & $\mathrm{O} 3-\mathrm{C} 3-\mathrm{Os} 1$ & $178.3(3)$ \\
\hline $\mathrm{O} 10-\mathrm{Os} 1-\mathrm{O} 8$ & $81.95(10)$ & $\mathrm{O} 4-\mathrm{C} 4-\mathrm{Os} 2$ & $178.5(3)$ \\
\hline C1-Os1-Os2 & $94.69(10)$ & O5-C5-Os2 & $177.4(3)$ \\
\hline $\mathrm{C} 1-\mathrm{Os} 1-\mathrm{O} 8$ & $95.42(13)$ & $\mathrm{O} 6-\mathrm{C} 6-\mathrm{Os} 2$ & $171.5(3)$ \\
\hline $\mathrm{C} 1-\mathrm{Os} 1-\mathrm{O} 10$ & $176.43(12)$ & $\mathrm{O} 7-\mathrm{C} 7-\mathrm{O} 8$ & $125.2(3)$ \\
\hline $\mathrm{C} 1-\mathrm{O} 1-\mathrm{C} 3$ & $93.66(15)$ & O7-C7-C8 & $117.2(3)$ \\
\hline C2-Os1-Os2 & $94.16(11)$ & $\mathrm{O} 8-\mathrm{C} 7-\mathrm{C} 8$ & $117.6(3)$ \\
\hline $\mathrm{C} 2-\mathrm{Os} 1-\mathrm{O} 8$ & $174.57(13)$ & $\mathrm{C} 7-\mathrm{C} 8-\mathrm{H} 8 \mathrm{~A}$ & 108.7 \\
\hline C2-Os1-O10 & $93.17(13)$ & $\mathrm{C} 7-\mathrm{C} 8-\mathrm{H} 8 \mathrm{~B}$ & 108.7 \\
\hline $\mathrm{C} 2-\mathrm{Os} 1-\mathrm{C} 1$ & 89.35 (16) & $\mathrm{C} 7-\mathrm{C} 8-\mathrm{C} 9$ & $114.2(3)$ \\
\hline C2-Os1-C3 & $95.16(15)$ & $\mathrm{H} 8 \mathrm{~A}-\mathrm{C} 8-\mathrm{H} 8 \mathrm{~B}$ & 107.6 \\
\hline C3-Os1-Os2 & $167.54(10)$ & $\mathrm{C} 9-\mathrm{C} 8-\mathrm{H} 8 \mathrm{~A}$ & 108.7 \\
\hline $\mathrm{C} 3-\mathrm{O} 1-\mathrm{O} 8$ & $87.14(12)$ & $\mathrm{C} 9-\mathrm{C} 8-\mathrm{H} 8 \mathrm{~B}$ & 108.7 \\
\hline C3-Os1-O10 & $88.64(12)$ & $\mathrm{C} 8-\mathrm{C} 9-\mathrm{H} 9 \mathrm{~A}$ & 109.5 \\
\hline O7-Os2-Os1 & $83.23(7)$ & $\mathrm{C} 8-\mathrm{C} 9-\mathrm{H} 9 \mathrm{~B}$ & 109.5 \\
\hline O7-Os2-O9 & $82.10(10)$ & $\mathrm{C} 8-\mathrm{C} 9-\mathrm{H} 9 \mathrm{C}$ & 109.5 \\
\hline O9-Os2-Os1 & $83.63(6)$ & $\mathrm{H} 9 \mathrm{~A}-\mathrm{C} 9-\mathrm{H} 9 \mathrm{~B}$ & 109.5 \\
\hline C4-Os2-Os1 & $91.60(10)$ & $\mathrm{H} 9 \mathrm{~A}-\mathrm{C} 9-\mathrm{H} 9 \mathrm{C}$ & 109.5 \\
\hline C4-Os2-O7 & $95.87(13)$ & $\mathrm{H} 9 \mathrm{~B}-\mathrm{C} 9-\mathrm{H} 9 \mathrm{C}$ & 109.5 \\
\hline C4-Os2-O9 & $174.99(12)$ & $\mathrm{O} 9-\mathrm{C} 10-\mathrm{C} 11$ & $118.1(3)$ \\
\hline $\mathrm{C} 4-\mathrm{Os} 2-\mathrm{C} 5$ & $90.30(15)$ & $\mathrm{O} 10-\mathrm{C} 10-\mathrm{O} 9$ & $125.7(3)$ \\
\hline C4-Os2-C6 & $91.42(14)$ & $\mathrm{O} 10-\mathrm{C} 10-\mathrm{C} 11$ & $116.2(3)$ \\
\hline C5-Os2-Os1 & $93.68(10)$ & $\mathrm{C} 10-\mathrm{C} 11-\mathrm{H} 11 \mathrm{~A}$ & 110.0 \\
\hline $\mathrm{C} 5-\mathrm{Os} 2-\mathrm{O} 7$ & $173.16(12)$ & $\mathrm{C} 10-\mathrm{C} 11-\mathrm{H} 11 \mathrm{~B}$ & 110.0 \\
\hline C5-Os2-O9 & $91.51(13)$ & $\mathrm{C} 10-\mathrm{C} 11-\mathrm{C} 12$ & $108.5(3)$ \\
\hline
\end{tabular}




\begin{tabular}{|c|c|c|c|}
\hline C5-Os2-C6 & $93.40(14)$ & $\begin{array}{l}\mathrm{H} 11 \mathrm{~A}-\mathrm{C} 11- \\
\mathrm{H} 11 \mathrm{~B}\end{array}$ & 108.4 \\
\hline C6-Os2-Os1 & $172.29(10)$ & $\mathrm{C} 12-\mathrm{C} 11-\mathrm{H} 11 \mathrm{~A}$ & 110.0 \\
\hline C6-Os2-O7 & $89.40(12)$ & $\mathrm{C} 12-\mathrm{C} 11-\mathrm{H} 11 \mathrm{~B}$ & 110.0 \\
\hline C6-Os2-O9 & $93.14(12)$ & $\mathrm{C} 11-\mathrm{C} 12-\mathrm{H} 12 \mathrm{~A}$ & 109.5 \\
\hline C7-O7-Os2 & $123.8(2)$ & $\mathrm{C} 11-\mathrm{C} 12-\mathrm{H} 12 \mathrm{~B}$ & 109.5 \\
\hline $\mathrm{C} 7-\mathrm{O} 8-\mathrm{Os} 1$ & $124.4(2)$ & $\mathrm{C} 11-\mathrm{C} 12-\mathrm{H} 12 \mathrm{C}$ & 109.5 \\
\hline C10-O9-Os2 & $122.9(2)$ & $\begin{array}{l}\mathrm{H} 12 \mathrm{~A}-\mathrm{C} 12- \\
\mathrm{H} 12 \mathrm{~B}\end{array}$ & 109.5 \\
\hline C10-O10-Os1 & $124.7(2)$ & $\begin{array}{l}\mathrm{H} 12 \mathrm{~A}-\mathrm{C} 12- \\
\mathrm{H} 12 \mathrm{C}\end{array}$ & 109.5 \\
\hline $\mathrm{O} 1-\mathrm{C} 1-\mathrm{Os} 1$ & $178.3(3)$ & $\begin{array}{l}\mathrm{H} 12 \mathrm{~B}-\mathrm{C} 12- \\
\mathrm{H} 12 \mathrm{C}\end{array}$ & 109.5 \\
\hline Os1-O8-C7-O7 & $4.6(5)$ & $\begin{array}{l}\text { Os2-O9-C10- } \\
\text { O10 }\end{array}$ & $3.7(5)$ \\
\hline $\mathrm{Os} 1-\mathrm{O} 8-\mathrm{C} 7-\mathrm{C} 8$ & $-174.2(2)$ & $\begin{array}{l}\text { Os2-O9-C10- } \\
\text { C11 }\end{array}$ & $-173.6(2)$ \\
\hline $\begin{array}{l}\text { Os1-O10-C10- } \\
\text { O9 }\end{array}$ & $-7.9(5)$ & $\mathrm{O} 7-\mathrm{C} 7-\mathrm{C} 8-\mathrm{C} 9$ & $178.5(4)$ \\
\hline $\begin{array}{l}\text { Os1-O10-C10- } \\
\text { C11 }\end{array}$ & $169.5(2)$ & $\mathrm{O} 8-\mathrm{C} 7-\mathrm{C} 8-\mathrm{C} 9$ & $-2.7(5)$ \\
\hline Os2-O7-C7-O8 & $-8.7(5)$ & $\begin{array}{l}\mathrm{O}-\mathrm{C} 10-\mathrm{C} 11- \\
\mathrm{C} 12\end{array}$ & $86.1(4)$ \\
\hline Os2-O7-C7-C8 & $170.1(2)$ & $\begin{array}{l}\mathrm{O} 10-\mathrm{C} 10-\mathrm{C} 11- \\
\mathrm{C} 12\end{array}$ & $-91.5(4)$ \\
\hline
\end{tabular}

(6)

Crystal data 


\begin{tabular}{|l|l|}
\hline $\mathrm{C}_{32} \mathrm{H}_{31} \mathrm{O}_{9} \mathrm{Os}_{2} \mathrm{P}$ & $Z=2$ \\
\hline$M_{r}=970.94$ & $F(000)=924$ \\
\hline Triclinic, $P \overline{1}$ & $D_{x}=1.998 \mathrm{Mg} \mathrm{m}^{-3}$ \\
\hline$a=10.0952(2) \AA$ & Cu Ka radiation, $\lambda=1.54184 \AA$ \\
\hline$b=11.6630(3) \AA$ & Cell parameters from 22340 reflections \\
\hline$C=13.7536(3) \AA$ & $\theta=3.8-73.3^{\circ}$ \\
\hline$\alpha=88.6185(19)^{\circ}$ & $\mu=15.55 \mathrm{~mm}^{-1}$ \\
\hline$\beta=86.7627(18)^{\circ}$ & $T=100 \mathrm{~K}$ \\
\hline$\gamma=86.9563(19)^{\circ}$ & Block, colorless \\
\hline$V=1614.12(6) \AA^{3}$ & $0.21 \times 0.14 \times 0.06 \mathrm{~mm}$ \\
\hline
\end{tabular}

\section{Data collection}

\begin{tabular}{|c|c|}
\hline $\begin{array}{l}\text { Rigaku SuperNova AtlasS2 CCD } \\
\text { diffractometer }\end{array}$ & 6461 independent reflections \\
\hline $\begin{array}{l}\text { Radiation source: micro-focus sealed X-ray } \\
\text { tube, SuperNova }(\mathrm{Cu}) \text { X-ray Source }\end{array}$ & 6196 reflections with $I>2 \sigma(I)$ \\
\hline Mirror monochromator & $R_{\text {int }}=0.038$ \\
\hline Detector resolution: 5.2387 pixels $\mathrm{mm}^{-1}$ & $\theta_{\max }=73.7^{\circ}, \theta_{\min }=3.8^{\circ}$ \\
\hline$\omega$ scans & $h=-12 \rightarrow 12$ \\
\hline $\begin{array}{l}\text { Absorption correction: multi-scan } \\
\text { CrysAlis PRO (Rigaku OD, 2015) }\end{array}$ & $k=-14 \rightarrow 14$ \\
\hline$T_{\min }=0.389, T_{\max }=1.000$ & $I=-17 \rightarrow 17$ \\
\hline 30757 measured reflections & \\
\hline
\end{tabular}

\section{Refinement}




\begin{tabular}{|l|l|}
\hline Least-squares matrix: full & $\begin{array}{l}\text { Hydrogen site location: inferred from } \\
\text { neighbouring sites }\end{array}$ \\
\hline$R\left[F^{2}>2 \sigma\left(F^{2}\right)\right]=0.019$ & $\mathrm{H}$-atom parameters constrained \\
\hline$W R\left(F^{2}\right)=0.046$ & $\begin{array}{c}w=1 /\left[\sigma^{2}\left(F_{0}^{2}\right)+(0.0202 P)^{2}+1.4592 P\right] \\
\text { where } P=\left(F_{0}^{2}+2 F_{\mathrm{c}}^{2}\right) / 3\end{array}$ \\
\hline$S=1.08$ & $(\Delta / \sigma)_{\max }=0.002$ \\
\hline 6461 reflections & $\Delta\rangle_{\max }=0.55$ e $\AA^{-3}$ \\
\hline 402 parameters & $\Delta\rangle_{\min }=-1.21 \mathrm{e} \AA^{-3}$ \\
\hline
\end{tabular}

\section{Special details}

Geometry. All esds (except the esd in the dihedral angle between two I.s. planes) are estimated using the full covariance matrix. The cell esds are taken into account individually in the estimation of esds in distances, angles and torsion angles; correlations between esds in cell parameters are only used when they are defined by crystal symmetry. An approximate (isotropic) treatment of cell esds is used for estimating esds involving I.s. planes.

Fractional atomic coordinates and isotropic or equivalent isotropic displacement parameters $\left(\AA^{2}\right)$ for (6)

\begin{tabular}{|l|l|l|l|l|}
\hline & $x$ & $y$ & $z$ & $U_{\text {iso }} / U_{\text {eq }}$ \\
\hline Os1 & $0.40363(2)$ & $0.24014(2)$ & $0.74050(2)$ & $0.01309(4)$ \\
\hline Os2 & $0.54412(2)$ & $0.18661(2)$ & $0.90234(2)$ & $0.01502(4)$ \\
\hline P1 & $0.24570(6)$ & $0.31901(6)$ & $0.62891(5)$ & $0.01422(13)$ \\
\hline O1 & $0.4095(2)$ & $0.00160(17)$ & $0.66128(18)$ & $0.0304(5)$ \\
\hline O2 & $0.6339(2)$ & $0.29582(19)$ & $0.60145(16)$ & $0.0265(5)$ \\
\hline O3 & $0.6587(2)$ & $-0.03401(19)$ & $0.81251(16)$ & $0.0290(5)$ \\
\hline O4 & $0.7726(2)$ & $0.31646(19)$ & $0.81165(17)$ & $0.0291(5)$ \\
\hline
\end{tabular}




\begin{tabular}{|l|l|l|l|l|}
\hline O5 & $0.6750(2)$ & $0.1397(2)$ & $1.09935(16)$ & $0.0305(5)$ \\
\hline O6 & $0.24276(18)$ & $0.20690(17)$ & $0.84390(14)$ & $0.0183(4)$ \\
\hline O7 & $0.36401(19)$ & $0.11713(17)$ & $0.95760(15)$ & $0.0208(4)$ \\
\hline O8 & $0.38423(19)$ & $0.40238(16)$ & $0.80787(14)$ & $0.0183(4)$ \\
\hline O9 & $0.44438(18)$ & $0.34012(17)$ & $0.95493(15)$ & $0.0194(4)$ \\
\hline C1 & $0.4078(3)$ & $0.0939(3)$ & $0.6913(2)$ & $0.0224(6)$ \\
\hline C2 & $0.5461(3)$ & $0.2749(2)$ & $0.6550(2)$ & $0.0198(6)$ \\
\hline C3 & $0.6153(3)$ & $0.0492(3)$ & $0.8479(2)$ & $0.0215(6)$ \\
\hline C4 & $0.6870(3)$ & $0.2654(3)$ & $0.8460(2)$ & $0.0213(6)$ \\
\hline C5 & $0.6204(3)$ & $0.1570(2)$ & $1.0308(2)$ & $0.0210(6)$ \\
\hline C6 & $0.2555(3)$ & $0.1515(2)$ & $0.9227(2)$ & $0.0204(6)$ \\
\hline C7 & $0.1308(3)$ & $0.1237(4)$ & $0.9828(3)$ & $0.0456(11)$ \\
\hline H7A & 0.125794 & 0.040890 & 0.986624 & $0.055^{\star}$ \\
\hline H7B & 0.138925 & 0.150066 & 1.048443 & $0.055^{\star}$ \\
\hline C8 & $0.0036(3)$ & $0.1740(4)$ & $0.9464(3)$ & $0.0415(9)$ \\
\hline H8A & -0.009125 & 0.144618 & 0.883182 & $0.062^{\star}$ \\
\hline H8B & 0.006905 & 0.256121 & 0.941912 & $0.062^{\star}$ \\
\hline H8C & -0.068809 & 0.153792 & 0.990605 & $0.062^{\star}$ \\
\hline C9 & $0.3874(3)$ & $0.4124(2)$ & $0.8986(2)$ & $0.0167(5)$ \\
\hline C10 & $0.3137(3)$ & $0.5149(2)$ & $0.9445(2)$ & $0.0222(6)$ \\
\hline H10A & 0.303259 & 0.575955 & 0.895932 & $0.027^{\star}$ \\
\hline H10B & 0.364372 & 0.542942 & 0.995984 & $0.027^{\star}$ \\
\hline C11 & $0.1783(3)$ & $0.4823(3)$ & $0.9860(3)$ & $0.0376(8)$ \\
\hline H11A & 0.126660 & 0.458409 & 0.934307 & $0.056^{\star}$ \\
\hline H11B & 0.547445 & 1.017205 & $0.056^{\star}$ \\
\hline & & & \\
\hline
\end{tabular}




\begin{tabular}{|l|l|l|l|l|}
\hline $\mathrm{H} 11 \mathrm{C}$ & 0.188798 & 0.420482 & 1.032689 & $0.056^{\star}$ \\
\hline $\mathrm{C} 12$ & $0.1795(3)$ & $0.4602(2)$ & $0.6651(2)$ & $0.0174(5)$ \\
\hline $\mathrm{C} 13$ & $0.0499(3)$ & $0.4791(3)$ & $0.7035(2)$ & $0.0254(6)$ \\
\hline $\mathrm{H} 13$ & -0.008069 & 0.419587 & 0.707137 & $0.030^{\star}$ \\
\hline $\mathrm{C} 14$ & $0.0072(3)$ & $0.5875(3)$ & $0.7366(3)$ & $0.0337(8)$ \\
\hline $\mathrm{H} 14$ & -0.079865 & 0.599664 & 0.761356 & $0.040^{\star}$ \\
\hline $\mathrm{C} 15$ & $0.0914(4)$ & $0.6779(3)$ & $0.7334(2)$ & $0.0321(7)$ \\
\hline $\mathrm{C} 16$ & $0.2206(3)$ & $0.6582(3)$ & $0.6962(2)$ & $0.0267(7)$ \\
\hline $\mathrm{H} 16$ & 0.278265 & 0.717901 & 0.693574 & $0.032^{\star}$ \\
\hline $\mathrm{C} 17$ & $0.2655(3)$ & $0.5516(2)$ & $0.6630(2)$ & $0.0211(6)$ \\
\hline $\mathrm{H} 17$ & 0.353074 & 0.539957 & 0.639044 & $0.025^{\star}$ \\
\hline $\mathrm{C} 18$ & $0.0429(5)$ & $0.7949(3)$ & $0.7678(3)$ & $0.0512(11)$ \\
\hline $\mathrm{H} 18 \mathrm{~A}$ & 0.069325 & 0.804868 & 0.832919 & $0.077^{\star}$ \\
\hline $\mathrm{H} 18 \mathrm{~B}$ & -0.052191 & 0.801978 & 0.767068 & $0.077^{\star}$ \\
\hline $\mathrm{H} 18 \mathrm{C}$ & 0.080683 & 0.852632 & 0.725414 & $0.077^{\star}$ \\
\hline $\mathrm{C} 19$ & $0.1010(3)$ & $0.2370(2)$ & $0.6101(2)$ & $0.0176(5)$ \\
\hline $\mathrm{C} 20$ & $0.0678(3)$ & $0.1458(3)$ & $0.6720(2)$ & $0.0265(7)$ \\
\hline $\mathrm{H} 20$ & 0.118338 & 0.127549 & 0.725229 & $0.032^{\star}$ \\
\hline $\mathrm{C} 21$ & $-0.0408(3)$ & $0.0815(3)$ & $0.6548(2)$ & $0.0285(7)$ \\
\hline $\mathrm{H} 21$ & -0.062350 & 0.021569 & 0.697637 & $0.034^{\star}$ \\
\hline $\mathrm{C} 22$ & $-0.1171(3)$ & $0.1047(3)$ & $0.5758(2)$ & $0.0218(6)$ \\
\hline $\mathrm{C} 23$ & $-0.0843(3)$ & $0.1965(3)$ & $0.5152(2)$ & $0.0278(7)$ \\
\hline $\mathrm{H} 23$ & -0.135113 & 0.214581 & 0.462034 & $0.033^{\star}$ \\
\hline $\mathrm{C} 24$ & $0.2622(3)$ & $0.5318(2)$ & $0.0257(7)$ \\
\hline $\mathrm{H} 24$ & 0.323730 & 0.490052 & $0.031^{\star}$ \\
\hline & & & & \\
\hline
\end{tabular}




\begin{tabular}{|l|l|l|l|l|}
\hline C25 & $-0.2292(3)$ & $0.0303(3)$ & $0.5551(2)$ & $0.0292(7)$ \\
\hline H25A & -0.194827 & -0.034993 & 0.518712 & $0.044^{\star}$ \\
\hline H25B & -0.292855 & 0.073834 & 0.517934 & $0.044^{\star}$ \\
\hline H25C & -0.271454 & 0.004677 & 0.615436 & $0.044^{\star}$ \\
\hline C26 & $0.3104(3)$ & $0.3386(2)$ & $0.5033(2)$ & $0.0160(5)$ \\
\hline C27 & $0.3794(3)$ & $0.2460(2)$ & $0.4584(2)$ & $0.0191(6)$ \\
\hline H27 & 0.403098 & 0.181038 & 0.495291 & $0.023^{\star}$ \\
\hline C28 & $0.4133(3)$ & $0.2495(3)$ & $0.3591(2)$ & $0.0223(6)$ \\
\hline H28 & 0.457846 & 0.186188 & 0.330034 & $0.027^{\star}$ \\
\hline C29 & $0.3814(3)$ & $0.3467(3)$ & $0.3027(2)$ & $0.0211(6)$ \\
\hline C30 & $0.3178(3)$ & $0.4399(2)$ & $0.3482(2)$ & $0.0222(6)$ \\
\hline H30 & 0.299088 & 0.506516 & 0.311874 & $0.027^{\star}$ \\
\hline C31 & $0.2810(3)$ & $0.4368(2)$ & $0.4474(2)$ & $0.0197(6)$ \\
\hline H31 & 0.236715 & 0.500351 & 0.476270 & $0.024^{\star}$ \\
\hline C32 & $0.4146(3)$ & $0.3481(3)$ & $0.1945(2)$ & $0.0300(7)$ \\
\hline H32A & 0.508795 & 0.335838 & 0.182509 & $0.045^{\star}$ \\
\hline H32B & 0.386097 & 0.421106 & 0.166994 & $0.045^{\star}$ \\
\hline H32C & 0.369938 & 0.288249 & 0.165069 & $0.045^{\star}$ \\
\hline & & & & \\
\hline
\end{tabular}

Atomic displacement parameters $\left(\AA^{2}\right)$ for (6)

\begin{tabular}{|l|l|l|l|l|l|l|}
\hline & $U^{11}$ & $U^{22}$ & $U^{\beta 3}$ & $U^{12}$ & $U^{13}$ & $U^{23}$ \\
\hline Os1 & $\begin{array}{l}0.01340 \\
(6)\end{array}$ & $\begin{array}{l}0.01470 \\
(6)\end{array}$ & $\begin{array}{l}0.01134 \\
(6)\end{array}$ & $\begin{array}{l}-0.00004 \\
(4)\end{array}$ & $\begin{array}{l}-0.00365 \\
(4)\end{array}$ & $\begin{array}{l}0.00142 \\
(4)\end{array}$ \\
\hline Os2 & $\begin{array}{l}0.01319 \\
(6)\end{array}$ & $\begin{array}{l}0.01902 \\
(7)\end{array}$ & $\begin{array}{l}0.01305 \\
(7)\end{array}$ & $\begin{array}{l}0.00051 \\
(4)\end{array}$ & $\begin{array}{l}-0.00461 \\
(4)\end{array}$ & $\begin{array}{l}0.00201 \\
(4)\end{array}$ \\
\hline P1 & $0.0142(3)$ & $0.0170(3)$ & $0.0116(3)$ & -0.0003 & -0.0032 & $0.0015(2)$ \\
\hline
\end{tabular}




\begin{tabular}{|c|c|c|c|c|c|c|}
\hline & & & & (2) & (2) & \\
\hline O1 & $\begin{array}{l}0.0403 \\
(13)\end{array}$ & $\begin{array}{l}0.0159 \\
(10)\end{array}$ & $\begin{array}{l}0.0364 \\
(13)\end{array}$ & $0.0034(9)$ & $\begin{array}{l}-0.0165 \\
(10)\end{array}$ & $\begin{array}{l}-0.0060 \\
(9)\end{array}$ \\
\hline $\mathrm{O} 2$ & $\begin{array}{l}0.0228 \\
(11)\end{array}$ & $\begin{array}{l}0.0313 \\
(12)\end{array}$ & $\begin{array}{l}0.0249 \\
(12)\end{array}$ & $\begin{array}{l}-0.0051 \\
(9)\end{array}$ & $0.0060(9)$ & $\begin{array}{l}-0.0002 \\
(9)\end{array}$ \\
\hline O3 & $\begin{array}{l}0.0367 \\
(12)\end{array}$ & $\begin{array}{l}0.0260 \\
(12)\end{array}$ & $\begin{array}{l}0.0240 \\
(12)\end{array}$ & $\begin{array}{l}0.0076 \\
(10)\end{array}$ & $\begin{array}{l}-0.0060 \\
(10)\end{array}$ & $\begin{array}{l}-0.0028 \\
(9)\end{array}$ \\
\hline $\mathrm{O} 4$ & $\begin{array}{l}0.0209 \\
(10)\end{array}$ & $\begin{array}{l}0.0339 \\
(12)\end{array}$ & $\begin{array}{l}0.0329 \\
(13)\end{array}$ & $\begin{array}{l}-0.0064 \\
(9)\end{array}$ & $\begin{array}{l}-0.0046 \\
(9)\end{array}$ & $\begin{array}{l}0.0088 \\
(10)\end{array}$ \\
\hline O5 & $\begin{array}{l}0.0317 \\
(12)\end{array}$ & $\begin{array}{l}0.0401 \\
(13)\end{array}$ & $\begin{array}{l}0.0203 \\
(12)\end{array}$ & $\begin{array}{l}0.0020 \\
(10)\end{array}$ & $\begin{array}{l}-0.0131 \\
(10)\end{array}$ & $0.0016(9)$ \\
\hline $\mathrm{O} 6$ & $0.0153(9)$ & $\begin{array}{l}0.0249 \\
(10)\end{array}$ & $\begin{array}{l}0.0147 \\
(10)\end{array}$ & $\begin{array}{l}-0.0018 \\
(8)\end{array}$ & $\begin{array}{l}-0.0030 \\
(7)\end{array}$ & $0.0033(8)$ \\
\hline $\mathrm{O} 7$ & $0.0173(9)$ & $\begin{array}{l}0.0278 \\
(11)\end{array}$ & $\begin{array}{l}0.0174 \\
(10)\end{array}$ & $\begin{array}{l}-0.0010 \\
(8)\end{array}$ & $\begin{array}{l}-0.0033 \\
(8)\end{array}$ & $0.0075(8)$ \\
\hline O8 & $\begin{array}{l}0.0212 \\
(10)\end{array}$ & $0.0171(9)$ & $\begin{array}{l}0.0170 \\
(10)\end{array}$ & $0.0010(8)$ & $\begin{array}{l}-0.0044 \\
(8)\end{array}$ & $\begin{array}{l}-0.0016 \\
(8)\end{array}$ \\
\hline O9 & $0.0179(9)$ & $\begin{array}{l}0.0230 \\
(10)\end{array}$ & $\begin{array}{l}0.0175 \\
(10)\end{array}$ & $0.0026(8)$ & $\begin{array}{l}-0.0054 \\
(8)\end{array}$ & $\begin{array}{l}-0.0017 \\
(8)\end{array}$ \\
\hline C1 & $\begin{array}{l}0.0200 \\
(14)\end{array}$ & $\begin{array}{l}0.0298 \\
(16)\end{array}$ & $\begin{array}{l}0.0176 \\
(14)\end{array}$ & $\begin{array}{l}0.0006 \\
(12)\end{array}$ & $\begin{array}{l}-0.0085 \\
(11)\end{array}$ & $\begin{array}{l}0.0091 \\
(12)\end{array}$ \\
\hline C2 & $\begin{array}{l}0.0249 \\
(14)\end{array}$ & $\begin{array}{l}0.0157 \\
(13)\end{array}$ & $\begin{array}{l}0.0198 \\
(14)\end{array}$ & $\begin{array}{l}0.0002 \\
(11)\end{array}$ & $\begin{array}{l}-0.0090 \\
(12)\end{array}$ & $\begin{array}{l}-0.0030 \\
(11)\end{array}$ \\
\hline C3 & $\begin{array}{l}0.0201 \\
(14)\end{array}$ & $\begin{array}{l}0.0272 \\
(16)\end{array}$ & $\begin{array}{l}0.0179 \\
(15)\end{array}$ & $\begin{array}{l}-0.0034 \\
(12)\end{array}$ & $\begin{array}{l}-0.0083 \\
(11)\end{array}$ & $\begin{array}{l}0.0072 \\
(12)\end{array}$ \\
\hline C4 & $\begin{array}{l}0.0201 \\
(14)\end{array}$ & $\begin{array}{l}0.0249 \\
(15)\end{array}$ & $\begin{array}{l}0.0191 \\
(15)\end{array}$ & $\begin{array}{l}0.0041 \\
(12)\end{array}$ & $\begin{array}{l}-0.0075 \\
(11)\end{array}$ & $\begin{array}{l}0.0011 \\
(11)\end{array}$ \\
\hline C5 & $\begin{array}{l}0.0189 \\
(13)\end{array}$ & $\begin{array}{l}0.0242 \\
(14)\end{array}$ & $\begin{array}{l}0.0197 \\
(15)\end{array}$ & $\begin{array}{l}0.0028 \\
(11)\end{array}$ & $\begin{array}{l}-0.0030 \\
(11)\end{array}$ & $\begin{array}{l}0.0001 \\
(11)\end{array}$ \\
\hline
\end{tabular}




\begin{tabular}{|c|c|c|c|c|c|c|}
\hline C6 & $\begin{array}{l}0.0170 \\
(13)\end{array}$ & $\begin{array}{l}0.0247 \\
(14)\end{array}$ & $\begin{array}{l}0.0193 \\
(15)\end{array}$ & $\begin{array}{l}-0.0013 \\
(11)\end{array}$ & $\begin{array}{l}-0.0008 \\
(11)\end{array}$ & $\begin{array}{l}0.0026 \\
(11)\end{array}$ \\
\hline $\mathrm{C} 7$ & $\begin{array}{l}0.0237 \\
(17)\end{array}$ & $0.067(3)$ & $0.044(2)$ & $\begin{array}{l}0.0000 \\
(17)\end{array}$ & $\begin{array}{l}0.0035 \\
(15)\end{array}$ & $0.031(2)$ \\
\hline C8 & $\begin{array}{l}0.0178 \\
(15)\end{array}$ & 0.064 (3) & $0.042(2)$ & $\begin{array}{l}-0.0049 \\
(16)\end{array}$ & $\begin{array}{l}0.0014 \\
(14)\end{array}$ & $\begin{array}{l}0.0141 \\
(19)\end{array}$ \\
\hline C9 & $\begin{array}{l}0.0127 \\
(12)\end{array}$ & $\begin{array}{l}0.0210 \\
(13)\end{array}$ & $\begin{array}{l}0.0168 \\
(14)\end{array}$ & $\begin{array}{l}-0.0021 \\
(10)\end{array}$ & $\begin{array}{l}-0.0025 \\
(10)\end{array}$ & $\begin{array}{l}-0.0025 \\
(10)\end{array}$ \\
\hline $\mathrm{C} 10$ & $\begin{array}{l}0.0202 \\
(14)\end{array}$ & $\begin{array}{l}0.0234 \\
(14)\end{array}$ & $\begin{array}{l}0.0232 \\
(15)\end{array}$ & $\begin{array}{l}0.0005 \\
(11)\end{array}$ & $\begin{array}{l}-0.0036 \\
(11)\end{array}$ & $\begin{array}{l}-0.0047 \\
(12)\end{array}$ \\
\hline C11 & $\begin{array}{l}0.0249 \\
(16)\end{array}$ & $0.041(2)$ & $0.046(2)$ & $\begin{array}{l}0.0017 \\
(14)\end{array}$ & $\begin{array}{l}0.0058 \\
(15)\end{array}$ & $\begin{array}{l}-0.0144 \\
(17)\end{array}$ \\
\hline C12 & $\begin{array}{l}0.0195 \\
(13)\end{array}$ & $\begin{array}{l}0.0205 \\
(13)\end{array}$ & $\begin{array}{l}0.0118 \\
(13)\end{array}$ & $\begin{array}{l}0.0054 \\
(11)\end{array}$ & $\begin{array}{l}-0.0043 \\
(10)\end{array}$ & $\begin{array}{l}0.0013 \\
(10)\end{array}$ \\
\hline $\mathrm{C} 13$ & $\begin{array}{l}0.0229 \\
(15)\end{array}$ & $\begin{array}{l}0.0332 \\
(17)\end{array}$ & $\begin{array}{l}0.0199 \\
(15)\end{array}$ & $\begin{array}{l}0.0019 \\
(12)\end{array}$ & $\begin{array}{l}-0.0016 \\
(12)\end{array}$ & $\begin{array}{l}-0.0039 \\
(12)\end{array}$ \\
\hline C14 & $\begin{array}{l}0.0277 \\
(16)\end{array}$ & $0.046(2)$ & $\begin{array}{l}0.0265 \\
(17)\end{array}$ & $\begin{array}{l}0.0140 \\
(15)\end{array}$ & $\begin{array}{l}-0.0017 \\
(13)\end{array}$ & $\begin{array}{l}-0.0100 \\
(15)\end{array}$ \\
\hline C15 & $0.047(2)$ & $\begin{array}{l}0.0279 \\
(16)\end{array}$ & $\begin{array}{l}0.0218 \\
(16)\end{array}$ & $\begin{array}{l}0.0122 \\
(14)\end{array}$ & $\begin{array}{l}-0.0142 \\
(14)\end{array}$ & $\begin{array}{l}-0.0063 \\
(13)\end{array}$ \\
\hline C16 & $\begin{array}{l}0.0395 \\
(17)\end{array}$ & $\begin{array}{l}0.0212 \\
(14)\end{array}$ & $\begin{array}{l}0.0204 \\
(15)\end{array}$ & $\begin{array}{l}0.0014 \\
(13)\end{array}$ & $\begin{array}{l}-0.0119 \\
(13)\end{array}$ & $\begin{array}{l}-0.0007 \\
(12)\end{array}$ \\
\hline C17 & $\begin{array}{l}0.0238 \\
(14)\end{array}$ & $\begin{array}{l}0.0208 \\
(14)\end{array}$ & $\begin{array}{l}0.0189 \\
(15)\end{array}$ & $\begin{array}{l}0.0027 \\
(11)\end{array}$ & $\begin{array}{l}-0.0063 \\
(11)\end{array}$ & $\begin{array}{l}0.0013 \\
(11)\end{array}$ \\
\hline C18 & $0.064(3)$ & $0.041(2)$ & 0.047 (3) & $0.025(2)$ & $-0.011(2)$ & $\begin{array}{l}-0.0175 \\
(18)\end{array}$ \\
\hline C19 & $\begin{array}{l}0.0140 \\
(12)\end{array}$ & $\begin{array}{l}0.0222 \\
(14)\end{array}$ & $\begin{array}{l}0.0165 \\
(14)\end{array}$ & $\begin{array}{l}-0.0007 \\
(10)\end{array}$ & $\begin{array}{l}-0.0015 \\
(10)\end{array}$ & $\begin{array}{l}-0.0009 \\
(11)\end{array}$ \\
\hline $\mathrm{C} 20$ & 0.0288 & 0.0280 & 0.0244 & -0.0087 & -0.0126 & 0.0075 \\
\hline
\end{tabular}




\begin{tabular}{|c|c|c|c|c|c|c|}
\hline & (16) & (16) & (16) & (13) & (13) & (13) \\
\hline C21 & $\begin{array}{l}0.0304 \\
(16)\end{array}$ & $\begin{array}{l}0.0268 \\
(16)\end{array}$ & $\begin{array}{l}0.0298 \\
(18)\end{array}$ & $\begin{array}{l}-0.0102 \\
(13)\end{array}$ & $\begin{array}{l}-0.0101 \\
(14)\end{array}$ & $\begin{array}{l}0.0095 \\
(13)\end{array}$ \\
\hline C22 & $\begin{array}{l}0.0175 \\
(13)\end{array}$ & $\begin{array}{l}0.0257 \\
(15)\end{array}$ & $\begin{array}{l}0.0228 \\
(15)\end{array}$ & $\begin{array}{l}-0.0023 \\
(11)\end{array}$ & $\begin{array}{l}-0.0016 \\
(11)\end{array}$ & $\begin{array}{l}-0.0057 \\
(12)\end{array}$ \\
\hline C23 & $\begin{array}{l}0.0201 \\
(14)\end{array}$ & $\begin{array}{l}0.0410 \\
(18)\end{array}$ & $\begin{array}{l}0.0233 \\
(16)\end{array}$ & $\begin{array}{l}-0.0052 \\
(13)\end{array}$ & $\begin{array}{l}-0.0088 \\
(12)\end{array}$ & $\begin{array}{l}0.0057 \\
(13)\end{array}$ \\
\hline C24 & $\begin{array}{l}0.0211 \\
(14)\end{array}$ & $\begin{array}{l}0.0327 \\
(16)\end{array}$ & $\begin{array}{l}0.0237 \\
(16)\end{array}$ & $\begin{array}{l}-0.0060 \\
(12)\end{array}$ & $\begin{array}{l}-0.0065 \\
(12)\end{array}$ & $\begin{array}{l}0.0108 \\
(13)\end{array}$ \\
\hline C25 & $\begin{array}{l}0.0247 \\
(15)\end{array}$ & $\begin{array}{l}0.0340 \\
(17)\end{array}$ & $\begin{array}{l}0.0307 \\
(18)\end{array}$ & $\begin{array}{l}-0.0091 \\
(13)\end{array}$ & $\begin{array}{l}-0.0064 \\
(13)\end{array}$ & $\begin{array}{l}-0.0077 \\
(14)\end{array}$ \\
\hline C26 & $\begin{array}{l}0.0133 \\
(12)\end{array}$ & $\begin{array}{l}0.0215 \\
(13)\end{array}$ & $\begin{array}{l}0.0135 \\
(13)\end{array}$ & $\begin{array}{l}-0.0032 \\
(10)\end{array}$ & $\begin{array}{l}-0.0019 \\
(10)\end{array}$ & $\begin{array}{l}0.0009 \\
(10)\end{array}$ \\
\hline C27 & $\begin{array}{l}0.0208 \\
(13)\end{array}$ & $\begin{array}{l}0.0187 \\
(13)\end{array}$ & $\begin{array}{l}0.0181 \\
(14)\end{array}$ & $\begin{array}{l}-0.0001 \\
(11)\end{array}$ & $\begin{array}{l}-0.0047 \\
(11)\end{array}$ & $\begin{array}{l}0.0021 \\
(11)\end{array}$ \\
\hline C28 & $\begin{array}{l}0.0238 \\
(14)\end{array}$ & $\begin{array}{l}0.0239 \\
(14)\end{array}$ & $\begin{array}{l}0.0192 \\
(15)\end{array}$ & $\begin{array}{l}0.0010 \\
(11)\end{array}$ & $\begin{array}{l}-0.0013 \\
(11)\end{array}$ & $\begin{array}{l}-0.0028 \\
(11)\end{array}$ \\
\hline C29 & $\begin{array}{l}0.0205 \\
(13)\end{array}$ & $\begin{array}{l}0.0280 \\
(15)\end{array}$ & $\begin{array}{l}0.0154 \\
(14)\end{array}$ & $\begin{array}{l}-0.0060 \\
(11)\end{array}$ & $\begin{array}{l}-0.0024 \\
(11)\end{array}$ & $\begin{array}{l}0.0002 \\
(11)\end{array}$ \\
\hline C30 & $\begin{array}{l}0.0268 \\
(15)\end{array}$ & $\begin{array}{l}0.0211 \\
(14)\end{array}$ & $\begin{array}{l}0.0192 \\
(15)\end{array}$ & $\begin{array}{l}-0.0035 \\
(12)\end{array}$ & $\begin{array}{l}-0.0046 \\
(12)\end{array}$ & $\begin{array}{l}0.0059 \\
(11)\end{array}$ \\
\hline C31 & $\begin{array}{l}0.0226 \\
(14)\end{array}$ & $\begin{array}{l}0.0196 \\
(13)\end{array}$ & $\begin{array}{l}0.0169 \\
(14)\end{array}$ & $\begin{array}{l}0.0003 \\
(11)\end{array}$ & $\begin{array}{l}-0.0021 \\
(11)\end{array}$ & $\begin{array}{l}0.0014 \\
(11)\end{array}$ \\
\hline C32 & $\begin{array}{l}0.0383 \\
(18)\end{array}$ & $\begin{array}{l}0.0344 \\
(17)\end{array}$ & $\begin{array}{l}0.0171 \\
(16)\end{array}$ & $\begin{array}{l}-0.0037 \\
(14)\end{array}$ & $\begin{array}{l}0.0026 \\
(13)\end{array}$ & $\begin{array}{l}-0.0016 \\
(13)\end{array}$ \\
\hline
\end{tabular}

Geometric parameters ( $(\AA, \stackrel{\circ}{)}$ for (6)

\begin{tabular}{|l|l|l|l|}
\hline Os1-Os2 & $2.7479(2)$ & C13-C14 & $1.392(5)$ \\
\hline Os1-P1 & $2.4075(6)$ & C14-H14 & 0.9300 \\
\hline
\end{tabular}




\begin{tabular}{|c|c|c|c|}
\hline Os1-O6 & $2.1422(19)$ & $\mathrm{C} 14-\mathrm{C} 15$ & $1.387(5)$ \\
\hline Os1-O8 & 2.1218 (19) & $\mathrm{C} 15-\mathrm{C} 16$ & $1.383(5)$ \\
\hline Os $1-\mathrm{C} 1$ & $1.848(3)$ & C15-C18 & $1.503(5)$ \\
\hline Os1-C2 & $1.861(3)$ & $\mathrm{C} 16-\mathrm{H} 16$ & 0.9300 \\
\hline Os2-O7 & $2.1239(19)$ & $\mathrm{C} 16-\mathrm{C} 17$ & $1.380(4)$ \\
\hline Os2-O9 & $2.129(2)$ & $\mathrm{C} 17-\mathrm{H} 17$ & 0.9300 \\
\hline Os2-C3 & $1.876(3)$ & $\mathrm{C} 18-\mathrm{H} 18 \mathrm{~A}$ & 0.9600 \\
\hline Os2-C4 & $1.872(3)$ & $\mathrm{C} 18-\mathrm{H} 18 \mathrm{~B}$ & 0.9600 \\
\hline Os2-C5 & $1.983(3)$ & $\mathrm{C} 18-\mathrm{H} 18 \mathrm{C}$ & 0.9600 \\
\hline $\mathrm{P} 1-\mathrm{C} 12$ & $1.814(3)$ & $\mathrm{C} 19-\mathrm{C} 20$ & $1.388(4)$ \\
\hline P1-C19 & $1.822(3)$ & $\mathrm{C} 19-\mathrm{C} 24$ & $1.391(4)$ \\
\hline $\mathrm{P} 1-\mathrm{C} 26$ & $1.825(3)$ & $\mathrm{C} 20-\mathrm{H} 20$ & 0.9300 \\
\hline $\mathrm{O} 1-\mathrm{C} 1$ & $1.161(4)$ & C20-C21 & $1.395(4)$ \\
\hline $\mathrm{O} 2-\mathrm{C} 2$ & $1.152(4)$ & $\mathrm{C} 21-\mathrm{H} 21$ & 0.9300 \\
\hline $\mathrm{O} 3-\mathrm{C} 3$ & $1.151(4)$ & C21-C22 & $1.380(4)$ \\
\hline $\mathrm{O} 4-\mathrm{C} 4$ & $1.148(4)$ & C22-C23 & $1.384(4)$ \\
\hline O5-C5 & $1.127(4)$ & C22-C25 & $1.506(4)$ \\
\hline $\mathrm{O} 6-\mathrm{C} 6$ & $1.257(3)$ & $\mathrm{C} 23-\mathrm{H} 23$ & 0.9300 \\
\hline O7-C6 & $1.263(3)$ & C23-C24 & $1.387(4)$ \\
\hline $\mathrm{O} 8-\mathrm{C} 9$ & 1.259 (3) & C24-H24 & 0.9300 \\
\hline O9-C9 & 1.268 (3) & $\mathrm{C} 25-\mathrm{H} 25 \mathrm{~A}$ & 0.9600 \\
\hline $\mathrm{C} 6-\mathrm{C} 7$ & $1.512(4)$ & $\mathrm{C} 25-\mathrm{H} 25 \mathrm{~B}$ & 0.9600 \\
\hline $\mathrm{C} 7-\mathrm{H} 7 \mathrm{~A}$ & 0.9700 & $\mathrm{C} 25-\mathrm{H} 25 \mathrm{C}$ & 0.9600 \\
\hline $\mathrm{C} 7-\mathrm{H} 7 \mathrm{~B}$ & 0.9700 & C26-C27 & $1.392(4)$ \\
\hline C7-C8 & $1.491(5)$ & $\mathrm{C} 26-\mathrm{C} 31$ & $1.392(4)$ \\
\hline
\end{tabular}




\begin{tabular}{|l|l|l|l|}
\hline C8-H8A & 0.9600 & C27-H27 & 0.9300 \\
\hline C8-H8B & 0.9600 & C27-C28 & $1.389(4)$ \\
\hline C8-H8C & 0.9600 & C28-H28 & 0.9300 \\
\hline C9-C10 & $1.507(4)$ & C28-C29 & $1.393(4)$ \\
\hline C10-H10A & 0.9700 & C29-C30 & $1.379(4)$ \\
\hline C10-H10B & 0.9700 & C29-C32 & $1.506(4)$ \\
\hline C10-C11 & $1.515(4)$ & C30-H30 & 0.9300 \\
\hline C11-H11A & 0.9600 & C30-C31 & $1.394(4)$ \\
\hline C11-H11B & 0.9600 & C31-H31 & 0.9300 \\
\hline C11-H11C & 0.9600 & C32-H32A & 0.9600 \\
\hline C12-C13 & $1.392(4)$ & C32-H32B & 0.9600 \\
\hline C12-C17 & $1.409(4)$ & C32-H32C & 0.9600 \\
\hline C13-H13 & 0.9300 & & 120.9 \\
\hline O & $89.68(13)$ & C14-C15-C18 & $120.9(4)$ \\
\hline P1-Os1-Os2 & $164.673(18)$ & H11B-C11- & 109.5 \\
\hline O6-Os1-Os2 & $80.26(5)$ & H11C & $122.2(2)$ \\
\hline O6-Os1-P1 & $89.31(5)$ & C13-C12-C17 & $118.6(3)$ \\
\hline O8-Os1-Os2 & $81.23(5)$ & C17-C12-P1 & $118.9(2)$ \\
\hline O8-Os1-P1 & $86.12(5)$ & C12-C13-H13 & 120.1 \\
\hline O8-Os1-O6 & $81.16(8)$ & C12-C13-C14 & $119.7(3)$ \\
\hline C1-Os1-Os2 & $97.27(8)$ & C14-C13-H13 & 120.1 \\
\hline C1-Os1-P1 & $94.45(8)$ & C13-C14-H14 & 119.2 \\
\hline C1-Os1-O6 & $93.03(11)$ & C15-C14-C13 & $121.7(3)$ \\
\hline C1-Os1-O8 & $174.16(11)$ & C15-C14-H14 & 119.2 \\
\hline C1-Os1-C2 & 89.21 & \\
\hline
\end{tabular}




\begin{tabular}{|l|l|l|l|}
\hline C2-Os1-Os2 & $98.10(8)$ & C16-C15-C14 & $118.3(3)$ \\
\hline C2-Os1-P1 & $91.79(8)$ & C16-C15-C18 & $120.8(3)$ \\
\hline C2-Os1-O6 & $176.99(10)$ & C15-C16-H16 & 119.4 \\
\hline C2-Os1-O8 & $96.11(10)$ & C17-C16-C15 & $121.3(3)$ \\
\hline O7-Os2-Os1 & $84.05(5)$ & C17-C16-H16 & 119.4 \\
\hline O7-Os2-O9 & $81.07(8)$ & C12-C17-H17 & 119.8 \\
\hline O9-Os2-Os1 & $82.62(5)$ & C16-C17-C12 & $120.4(3)$ \\
\hline C3-Os2-Os1 & $91.82(8)$ & C16-C17-H17 & 119.8 \\
\hline C3-Os2-O7 & $95.21(10)$ & C15-C18-H18A & 109.5 \\
\hline C3-Os2-O9 & $173.58(9)$ & C15-C18-H18B & 109.5 \\
\hline C3-Os2-C5 & $94.90(12)$ & C15-C18-H18C & 109.5 \\
\hline C4-Os2-Os1 & $89.46(8)$ & $\begin{array}{l}\text { H18A-C18- } \\
\text { H18B }\end{array}$ & 109.5 \\
\hline C4-Os2-O7 & $171.57(10)$ & $\begin{array}{l}\text { H18A-C18- } \\
\text { H18C }\end{array}$ & 109.5 \\
\hline C4-Os2-O9 & $92.79(11)$ & $\begin{array}{l}\text { H18B-C18- } \\
\text { H18C }\end{array}$ & 109.5 \\
\hline C4-Os2-C3 & $90.36(13)$ & C20-C19-P1 & $121.1(2)$ \\
\hline C4-Os2-C5 & $96.32(12)$ & C20-C19-C24 & $118.0(2)$ \\
\hline C5-Os2-Os1 & $171.10(9)$ & C24-C19-P1 & $120.8(2)$ \\
\hline C5-Os2-O7 & $89.52(10)$ & C19-C20-H20 & 119.8 \\
\hline C5-Os2-O9 & $90.31(10)$ & C19-C20-C21 & $120.4(3)$ \\
\hline C12-P1-Os1 & $111.59(9)$ & C21-C20-H20 & 119.8 \\
\hline C12-P1-C19 & $105.33(13)$ & C20-C21-H21 & 119.2 \\
\hline C12-P1-C26 & $104.93(13)$ & C22-C21-C20 & $121.6(3)$ \\
\hline C19-P1-Os1 & $117.64(9)$ & C22-C21-H21 & 119.2 \\
\hline
\end{tabular}




\begin{tabular}{|c|c|c|c|}
\hline $\mathrm{C} 19-\mathrm{P} 1-\mathrm{C} 26$ & $100.50(12)$ & $\mathrm{C} 21-\mathrm{C} 22-\mathrm{C} 23$ & $117.6(3)$ \\
\hline $\mathrm{C} 26-\mathrm{P} 1-\mathrm{Os} 1$ & $115.42(9)$ & C21-C22-C25 & $121.0(3)$ \\
\hline $\mathrm{C} 6-\mathrm{O} 6-\mathrm{Os} 1$ & $124.22(18)$ & C23-C22-C25 & $121.5(3)$ \\
\hline C6-O7-Os2 & $119.70(18)$ & $\mathrm{C} 22-\mathrm{C} 23-\mathrm{H} 23$ & 119.2 \\
\hline C9-O8-Os1 & $122.11(18)$ & $\mathrm{C} 22-\mathrm{C} 23-\mathrm{C} 24$ & $121.6(3)$ \\
\hline C9-O9-Os2 & $121.98(19)$ & $\mathrm{C} 24-\mathrm{C} 23-\mathrm{H} 23$ & 119.2 \\
\hline $\mathrm{O} 1-\mathrm{C} 1-\mathrm{Os} 1$ & $179.2(3)$ & $\mathrm{C} 19-\mathrm{C} 24-\mathrm{H} 24$ & 119.6 \\
\hline $\mathrm{O} 2-\mathrm{C} 2-\mathrm{Os} 1$ & $179.4(3)$ & $\mathrm{C} 23-\mathrm{C} 24-\mathrm{C} 19$ & $120.7(3)$ \\
\hline O3-C3-Os2 & $178.5(2)$ & $\mathrm{C} 23-\mathrm{C} 24-\mathrm{H} 24$ & 119.6 \\
\hline O4-C4-Os2 & $178.1(3)$ & $\mathrm{C} 22-\mathrm{C} 25-\mathrm{H} 25 \mathrm{~A}$ & 109.5 \\
\hline O5-C5-Os2 & $173.6(3)$ & $\mathrm{C} 22-\mathrm{C} 25-\mathrm{H} 25 \mathrm{~B}$ & 109.5 \\
\hline $\mathrm{O} 6-\mathrm{C} 6-\mathrm{O} 7$ & $125.9(3)$ & $\mathrm{C} 22-\mathrm{C} 25-\mathrm{H} 25 \mathrm{C}$ & 109.5 \\
\hline $\mathrm{O} 6-\mathrm{C} 6-\mathrm{C} 7$ & $118.0(3)$ & $\begin{array}{l}\mathrm{H} 25 \mathrm{~A}-\mathrm{C} 25- \\
\mathrm{H} 25 \mathrm{~B}\end{array}$ & 109.5 \\
\hline $\mathrm{O} 7-\mathrm{C} 6-\mathrm{C} 7$ & $116.0(3)$ & $\begin{array}{l}\mathrm{H} 25 \mathrm{~A}-\mathrm{C} 25- \\
\mathrm{H} 25 \mathrm{C}\end{array}$ & 109.5 \\
\hline $\mathrm{C} 6-\mathrm{C} 7-\mathrm{H} 7 \mathrm{~A}$ & 108.3 & $\begin{array}{l}\mathrm{H} 25 \mathrm{~B}-\mathrm{C} 25- \\
\mathrm{H} 25 \mathrm{C}\end{array}$ & 109.5 \\
\hline $\mathrm{C} 6-\mathrm{C} 7-\mathrm{H} 7 \mathrm{~B}$ & 108.3 & $\mathrm{C} 27-\mathrm{C} 26-\mathrm{P} 1$ & $118.1(2)$ \\
\hline $\mathrm{H} 7 \mathrm{~A}-\mathrm{C} 7-\mathrm{H} 7 \mathrm{~B}$ & 107.4 & $\mathrm{C} 31-\mathrm{C} 26-\mathrm{P} 1$ & $122.9(2)$ \\
\hline $\mathrm{C} 8-\mathrm{C} 7-\mathrm{C} 6$ & $115.9(3)$ & $\mathrm{C} 31-\mathrm{C} 26-\mathrm{C} 27$ & $118.6(3)$ \\
\hline $\mathrm{C} 8-\mathrm{C} 7-\mathrm{H} 7 \mathrm{~A}$ & 108.3 & $\mathrm{C} 26-\mathrm{C} 27-\mathrm{H} 27$ & 119.6 \\
\hline $\mathrm{C} 8-\mathrm{C} 7-\mathrm{H} 7 \mathrm{~B}$ & 108.3 & C28-C27-C26 & $120.8(3)$ \\
\hline $\mathrm{C} 7-\mathrm{C} 8-\mathrm{H} 8 \mathrm{~A}$ & 109.5 & $\mathrm{C} 28-\mathrm{C} 27-\mathrm{H} 27$ & 119.6 \\
\hline $\mathrm{C} 7-\mathrm{C} 8-\mathrm{H} 8 \mathrm{~B}$ & 109.5 & $\mathrm{C} 27-\mathrm{C} 28-\mathrm{H} 28$ & 119.7 \\
\hline $\mathrm{C} 7-\mathrm{C} 8-\mathrm{H} 8 \mathrm{C}$ & 109.5 & C27-C28-C29 & $120.7(3)$ \\
\hline
\end{tabular}




\begin{tabular}{|c|c|c|c|}
\hline $\mathrm{H} 8 \mathrm{~A}-\mathrm{C} 8-\mathrm{H} 8 \mathrm{~B}$ & 109.5 & $\mathrm{C} 29-\mathrm{C} 28-\mathrm{H} 28$ & 119.7 \\
\hline $\mathrm{H} 8 \mathrm{~A}-\mathrm{C} 8-\mathrm{H} 8 \mathrm{C}$ & 109.5 & $\mathrm{C} 28-\mathrm{C} 29-\mathrm{C} 32$ & $120.3(3)$ \\
\hline $\mathrm{H} 8 \mathrm{~B}-\mathrm{C} 8-\mathrm{H} 8 \mathrm{C}$ & 109.5 & C30-C29-C28 & $118.3(3)$ \\
\hline $\mathrm{O} 8-\mathrm{C} 9-\mathrm{O} 9$ & $124.7(3)$ & C30-C29-C32 & $121.4(3)$ \\
\hline $\mathrm{O} 8-\mathrm{C} 9-\mathrm{C} 10$ & $117.8(2)$ & $\mathrm{C} 29-\mathrm{C} 30-\mathrm{H} 30$ & 119.2 \\
\hline $\mathrm{O} 9-\mathrm{C} 9-\mathrm{C} 10$ & $117.4(3)$ & $\mathrm{C} 29-\mathrm{C} 30-\mathrm{C} 31$ & $121.6(3)$ \\
\hline $\mathrm{C} 9-\mathrm{C} 10-\mathrm{H} 10 \mathrm{~A}$ & 109.6 & $\mathrm{C} 31-\mathrm{C} 30-\mathrm{H} 30$ & 119.2 \\
\hline $\mathrm{C} 9-\mathrm{C} 10-\mathrm{H} 10 \mathrm{~B}$ & 109.6 & $\mathrm{C} 26-\mathrm{C} 31-\mathrm{C} 30$ & $120.0(3)$ \\
\hline $\mathrm{C} 9-\mathrm{C} 10-\mathrm{C} 11$ & $110.2(2)$ & $\mathrm{C} 26-\mathrm{C} 31-\mathrm{H} 31$ & 120.0 \\
\hline $\mathrm{H} 10 \mathrm{~A}-\mathrm{C} 10-\mathrm{H} 10 \mathrm{~B}$ & 108.1 & $\mathrm{C} 30-\mathrm{C} 31-\mathrm{H} 31$ & 120.0 \\
\hline $\mathrm{C} 11-\mathrm{C} 10-\mathrm{H} 10 \mathrm{~A}$ & 109.6 & $\mathrm{C} 29-\mathrm{C} 32-\mathrm{H} 32 \mathrm{~A}$ & 109.5 \\
\hline $\mathrm{C} 11-\mathrm{C} 10-\mathrm{H} 10 \mathrm{~B}$ & 109.6 & $\mathrm{C} 29-\mathrm{C} 32-\mathrm{H} 32 \mathrm{~B}$ & 109.5 \\
\hline $\mathrm{C} 10-\mathrm{C} 11-\mathrm{H} 11 \mathrm{~A}$ & 109.5 & $\mathrm{C} 29-\mathrm{C} 32-\mathrm{H} 32 \mathrm{C}$ & 109.5 \\
\hline $\mathrm{C} 10-\mathrm{C} 11-\mathrm{H} 11 \mathrm{~B}$ & 109.5 & $\begin{array}{l}\mathrm{H} 32 \mathrm{~A}-\mathrm{C} 32- \\
\mathrm{H} 32 \mathrm{~B}\end{array}$ & 109.5 \\
\hline $\mathrm{C} 10-\mathrm{C} 11-\mathrm{H} 11 \mathrm{C}$ & 109.5 & $\begin{array}{l}\mathrm{H} 32 \mathrm{~A}-\mathrm{C} 32- \\
\mathrm{H} 32 \mathrm{C}\end{array}$ & 109.5 \\
\hline $\mathrm{H} 11 \mathrm{~A}-\mathrm{C} 11-\mathrm{H} 11 \mathrm{~B}$ & 109.5 & $\begin{array}{l}\mathrm{H} 32 \mathrm{~B}-\mathrm{C} 32- \\
\mathrm{H} 32 \mathrm{C}\end{array}$ & 109.5 \\
\hline $\mathrm{H} 11 \mathrm{~A}-\mathrm{C} 11-\mathrm{H} 11 \mathrm{C}$ & 109.5 & & \\
\hline $\begin{array}{l}\text { Os1-P1-C12- } \\
\mathrm{C} 13\end{array}$ & $-107.8(2)$ & $\begin{array}{l}\text { C13-C14-C15- } \\
\text { C16 }\end{array}$ & $0.0(5)$ \\
\hline $\begin{array}{l}\text { Os1-P1-C12- } \\
\text { C17 }\end{array}$ & $66.0(2)$ & $\begin{array}{l}\text { C13-C14-C15- } \\
\text { C18 }\end{array}$ & $178.9(3)$ \\
\hline $\begin{array}{l}\text { Os1-P1-C19- } \\
\mathrm{C} 20\end{array}$ & $13.3(3)$ & $\begin{array}{l}\mathrm{C} 14-\mathrm{C} 15-\mathrm{C} 16- \\
\mathrm{C} 17\end{array}$ & $0.0(5)$ \\
\hline
\end{tabular}




\begin{tabular}{|c|c|c|c|}
\hline $\begin{array}{l}\text { Os1-P1-C19- } \\
\text { C24 }\end{array}$ & $-165.1(2)$ & $\begin{array}{l}\mathrm{C} 15-\mathrm{C} 16-\mathrm{C} 17- \\
\mathrm{C} 12\end{array}$ & $0.8(4)$ \\
\hline $\begin{array}{l}\text { Os1-P1-C26- } \\
\text { C27 }\end{array}$ & $50.8(2)$ & $\begin{array}{l}\text { C17-C12-C13- } \\
\text { C14 }\end{array}$ & $1.5(4)$ \\
\hline $\begin{array}{l}\text { Os1-P1-C26- } \\
\text { C31 }\end{array}$ & $-136.7(2)$ & $\begin{array}{l}\mathrm{C} 18-\mathrm{C} 15-\mathrm{C} 16- \\
\mathrm{C} 17\end{array}$ & $-178.9(3)$ \\
\hline Os1-O6-C6-O7 & $7.4(4)$ & $\begin{array}{l}\mathrm{C} 19-\mathrm{P} 1-\mathrm{C} 12- \\
\mathrm{C} 13\end{array}$ & $20.9(3)$ \\
\hline $\mathrm{Os} 1-\mathrm{O} 6-\mathrm{C} 6-\mathrm{C} 7$ & $-173.3(3)$ & $\begin{array}{l}\mathrm{C} 19-\mathrm{P} 1-\mathrm{C} 12- \\
\mathrm{C} 17\end{array}$ & $-165.2(2)$ \\
\hline Os1-O8-C9-O9 & $25.0(3)$ & $\begin{array}{l}\text { C19-P1-C26- } \\
\text { C27 }\end{array}$ & $-76.8(2)$ \\
\hline Os $1-O 8-C 9-C 10$ & $-152.37(18)$ & $\begin{array}{l}\mathrm{C} 19-\mathrm{P} 1-\mathrm{C} 26- \\
\mathrm{C} 31\end{array}$ & $95.7(2)$ \\
\hline Os2-O7-C6-O6 & $15.6(4)$ & $\begin{array}{l}\mathrm{C} 19-\mathrm{C} 20-\mathrm{C} 21- \\
\mathrm{C} 22\end{array}$ & $1.0(6)$ \\
\hline Os2-O7-C6-C7 & $-163.7(3)$ & $\begin{array}{l}\mathrm{C} 20-\mathrm{C} 19-\mathrm{C} 24- \\
\mathrm{C} 23\end{array}$ & $-1.2(5)$ \\
\hline Os2-O9-C9-O8 & $-0.6(4)$ & $\begin{array}{l}\mathrm{C} 20-\mathrm{C} 21-\mathrm{C} 22- \\
\mathrm{C} 23\end{array}$ & $-1.8(5)$ \\
\hline Os2-O9-C9-C10 & $176.83(17)$ & $\begin{array}{l}\mathrm{C} 20-\mathrm{C} 21-\mathrm{C} 22- \\
\mathrm{C} 25\end{array}$ & $176.5(3)$ \\
\hline $\begin{array}{l}\mathrm{P} 1-\mathrm{C} 12-\mathrm{C} 13- \\
\mathrm{C} 14\end{array}$ & $175.4(2)$ & $\begin{array}{l}\text { C21-C22-C23- } \\
\text { C24 }\end{array}$ & $1.0(5)$ \\
\hline $\begin{array}{l}\mathrm{P} 1-\mathrm{C} 12-\mathrm{C} 17- \\
\mathrm{C} 16\end{array}$ & $-175.6(2)$ & $\begin{array}{l}\mathrm{C} 22-\mathrm{C} 23-\mathrm{C} 24- \\
\mathrm{C} 19\end{array}$ & $0.5(5)$ \\
\hline $\begin{array}{l}\mathrm{P} 1-\mathrm{C} 19-\mathrm{C} 20- \\
\mathrm{C} 21\end{array}$ & $-177.9(3)$ & $\begin{array}{l}\mathrm{C} 24-\mathrm{C} 19-\mathrm{C} 20- \\
\mathrm{C} 21\end{array}$ & $0.5(5)$ \\
\hline $\mathrm{P} 1-\mathrm{C} 19-\mathrm{C} 24-$ & $177.2(3)$ & $\mathrm{C} 25-\mathrm{C} 22-\mathrm{C} 23-$ & $-177.3(3)$ \\
\hline
\end{tabular}




\begin{tabular}{|c|c|c|c|}
\hline C23 & & C24 & \\
\hline $\begin{array}{l}\mathrm{P} 1-\mathrm{C} 26-\mathrm{C} 27- \\
\mathrm{C} 28\end{array}$ & $170.1(2)$ & $\begin{array}{l}\mathrm{C} 26-\mathrm{P} 1-\mathrm{C} 12- \\
\mathrm{C} 13\end{array}$ & $126.5(2)$ \\
\hline $\begin{array}{l}\mathrm{P} 1-\mathrm{C} 26-\mathrm{C} 31- \\
\mathrm{C} 30\end{array}$ & $-171.0(2)$ & $\begin{array}{l}\mathrm{C} 26-\mathrm{P} 1-\mathrm{C} 12- \\
\mathrm{C} 17\end{array}$ & $-59.7(2)$ \\
\hline $\mathrm{O} 6-\mathrm{C} 6-\mathrm{C} 7-\mathrm{C} 8$ & $-5.1(5)$ & $\begin{array}{l}\text { C26-P1-C19- } \\
\text { C20 }\end{array}$ & $139.4(3)$ \\
\hline $\mathrm{O} 7-\mathrm{C} 6-\mathrm{C} 7-\mathrm{C} 8$ & $174.3(3)$ & $\begin{array}{l}\text { C26-P1-C19- } \\
\text { C24 }\end{array}$ & $-38.9(3)$ \\
\hline $\mathrm{O} 8-\mathrm{C} 9-\mathrm{C} 10-\mathrm{C} 11$ & $97.4(3)$ & $\begin{array}{l}\text { C26-C27-C28- } \\
\text { C29 }\end{array}$ & $1.3(4)$ \\
\hline $\mathrm{O} 9-\mathrm{C} 9-\mathrm{C} 10-\mathrm{C} 11$ & $-80.2(3)$ & $\begin{array}{l}\text { C27-C26-C31- } \\
\text { C30 }\end{array}$ & $1.5(4)$ \\
\hline $\begin{array}{l}\mathrm{C} 12-\mathrm{P} 1-\mathrm{C} 19- \\
\mathrm{C} 20\end{array}$ & $-111.7(3)$ & $\begin{array}{l}\text { C27-C28-C29- } \\
\text { C30 }\end{array}$ & $1.3(4)$ \\
\hline $\begin{array}{l}\mathrm{C} 12-\mathrm{P} 1-\mathrm{C} 19- \\
\mathrm{C} 24\end{array}$ & 69.9 (3) & $\begin{array}{l}\text { C27-C28-C29- } \\
\text { C32 }\end{array}$ & $-178.0(3)$ \\
\hline $\begin{array}{l}\mathrm{C} 12-\mathrm{P} 1-\mathrm{C} 26- \\
\mathrm{C} 27\end{array}$ & $174.1(2)$ & $\begin{array}{l}\mathrm{C} 28-\mathrm{C} 29-\mathrm{C} 30- \\
\mathrm{C} 31\end{array}$ & $-2.6(4)$ \\
\hline $\begin{array}{l}\mathrm{C} 12-\mathrm{P} 1-\mathrm{C} 26- \\
\mathrm{C} 31\end{array}$ & $-13.4(2)$ & $\begin{array}{l}\text { C29-C30-C31- } \\
\text { C26 }\end{array}$ & $1.2(4)$ \\
\hline $\begin{array}{l}\text { C12-C13-C14- } \\
\text { C15 }\end{array}$ & $-0.8(5)$ & $\begin{array}{l}\text { C31-C26-C27- } \\
\text { C28 }\end{array}$ & $-2.7(4)$ \\
\hline $\begin{array}{l}\text { C13-C12-C17- } \\
\text { C16 }\end{array}$ & $-1.6(4)$ & $\begin{array}{l}\text { C32-C29-C30- } \\
\text { C31 }\end{array}$ & $176.7(3)$ \\
\hline
\end{tabular}

\section{(7) $\cdot \mathrm{CH}_{2} \mathrm{Cl}_{2}$}

Crystal data 


\begin{tabular}{|l|l|}
\hline$M_{r}=1332.20$ & $D_{x}=1.712 \mathrm{Mg} \mathrm{m}^{-3}$ \\
\hline Monoclinic, $P 2_{1} / c$ & Mo Ka radiation, $\lambda=0.71073 \AA$ \\
\hline$a=18.4968(6) \AA$ & Cell parameters from 11533 reflections \\
\hline$b=18.7369(7) \AA$ & $\theta=1.6-27.6^{\circ}$ \\
\hline$C=14.9214(6) \AA$ & $\mu=5.13 \mathrm{~mm}^{-1}$ \\
\hline$\beta=91.806(2)^{\circ}$ & $T=100 \mathrm{~K}$ \\
\hline$V=5168.8(3) \AA^{3}$ & Prisms, colorless \\
\hline$Z=4$ & $0.28 \times 0.12 \times 0.09 \mathrm{~mm}$ \\
\hline
\end{tabular}

Data collection

\begin{tabular}{|c|c|}
\hline $\begin{array}{l}\text { Rigaku SCX-Mini Mercury 2+ CCD } \\
\text { diffractometer }\end{array}$ & 9639 reflections with $I>2 \sigma(I)$ \\
\hline Radiation source: sealed tube & $R_{\text {int }}=0.060$ \\
\hline$\omega$-scans & $\theta_{\max }=27.5^{\circ}, \theta_{\min }=3.1^{\circ}$ \\
\hline $\begin{array}{l}\text { Absorption correction: multi-scan } \\
\text { ABSCOR (Higashi, 2001) }\end{array}$ & $h=-24 \rightarrow 24$ \\
\hline$T_{\min }=0.673, T_{\max }=1.00$ & $k=-23 \rightarrow 24$ \\
\hline 54214 measured reflections & $I=-19 \rightarrow 19$ \\
\hline 11794 independent reflections & \\
\hline
\end{tabular}

Refinement

\begin{tabular}{|l|l|}
\hline Refinement on $F^{2}$ & 20 restraints \\
\hline Least-squares matrix: full & Hydrogen site location: mixed \\
\hline$R\left[F^{2}>2 \sigma\left(F^{2}\right)\right]=0.036$ & H-atom parameters constrained \\
\hline$W R\left(F^{2}\right)=0.071$ & $\begin{array}{c}W=1 /\left[\sigma^{2}\left(F_{0}^{2}\right)+(0.0224 P)^{2}+9.8357 P\right] \\
\text { where } P=\left(F_{0}^{2}+2 F_{c}^{2}\right) / 3\end{array}$ \\
\hline
\end{tabular}




\begin{tabular}{|l|l|}
\hline$S=1.07$ & $(\Delta / \sigma)_{\max }=0.003$ \\
\hline 11794 reflections & $\Delta\rangle_{\max }=1.39 \mathrm{e} \AA^{-3}$ \\
\hline 624 parameters & $\Delta\rangle_{\min }=-1.32 \mathrm{e} \AA^{-3}$ \\
\hline
\end{tabular}

Special details

Geometry. All esds (except the esd in the dihedral angle between two I.s. planes) are estimated using the full covariance matrix. The cell esds are taken into account individually in the estimation of esds in distances, angles and torsion angles; correlations between esds in cell parameters are only used when they are defined by crystal symmetry. An approximate (isotropic) treatment of cell esds is used for estimating esds involving I.s. planes.

Fractional atomic coordinates and isotropic or equivalent isotropic displacement parameters $\left(\AA^{2}\right)$ for (7) $\cdot \mathrm{CH}_{2} \mathrm{Cl}_{2}$

\begin{tabular}{|l|l|l|l|l|l|}
\hline & $x$ & $y$ & $z$ & $U_{\text {iso }} / U_{\text {eq }}$ & Occ. $(<1)$ \\
\hline C1 & $0.7088(2)$ & $0.4345(2)$ & $-0.0552(3)$ & $0.0150(9)$ & \\
\hline C2 & $0.6426(2)$ & $0.4242(2)$ & $-0.0150(3)$ & $0.0218(10)$ & \\
\hline H2 & 0.6372 & 0.4380 & 0.0457 & $0.026^{\star}$ & \\
\hline C3 & $0.5843(3)$ & $0.3938(3)$ & $-0.0628(3)$ & $0.0248(11)$ & \\
\hline H3 & 0.5397 & 0.3874 & -0.0340 & $0.030^{*}$ & \\
\hline C4 & $0.5903(2)$ & $0.3728(2)$ & $-0.1516(3)$ & $0.0202(10)$ & \\
\hline C5 & $0.6558(3)$ & $0.3852(3)$ & $-0.1917(3)$ & $0.0247(11)$ & \\
\hline H5 & 0.6608 & 0.3727 & -0.2529 & $0.030^{\star}$ & \\
\hline C6 & $0.7143(3)$ & $0.4154(2)$ & $-0.1445(3)$ & $0.0216(10)$ & \\
\hline H6 & 0.7585 & 0.4231 & -0.1738 & $0.026^{\star}$ & \\
\hline C7 & $0.5284(2)$ & $0.3369(3)$ & $-0.2015(3)$ & $0.0236(11)$ & \\
\hline H7A & 0.5338 & 0.2850 & -0.1967 & $0.035^{\star}$ & \\
\hline
\end{tabular}




\begin{tabular}{|l|l|l|l|l|l|}
\hline H7B & 0.5285 & 0.3509 & -0.2648 & $0.035^{\star}$ & \\
\hline H7C & 0.4826 & 0.3513 & -0.1756 & $0.035^{\star}$ & \\
\hline C8 & $0.8199(2)$ & $0.5373(2)$ & $-0.0699(3)$ & $0.0153(9)$ & \\
\hline C9 & $0.8615(2)$ & $0.5187(2)$ & $-0.1427(3)$ & $0.0180(9)$ & \\
\hline H9 & 0.8780 & 0.4710 & -0.1488 & $0.022^{\star}$ & \\
\hline C10 & $0.8790(2)$ & $0.5699(2)$ & $-0.2067(3)$ & $0.0190(10)$ & \\
\hline H10 & 0.9057 & 0.5557 & -0.2571 & $0.023^{\star}$ & \\
\hline C11 & $0.8585(2)$ & $0.6407(2)$ & $-0.1988(3)$ & $0.0188(10)$ & \\
\hline C12 & $0.8190(2)$ & $0.6591(2)$ & $-0.1246(3)$ & $0.0176(10)$ & \\
\hline H12 & 0.8053 & 0.7074 & -0.1165 & $0.021^{\star}$ & \\
\hline C13 & $0.7991(2)$ & $0.6088(2)$ & $-0.0624(3)$ & $0.0160(9)$ & \\
\hline H13 & 0.7707 & 0.6230 & -0.0135 & $0.019^{\star}$ & \\
\hline C14 & $0.8760(3)$ & $0.6936(3)$ & $-0.2702(3)$ & $0.0281(12)$ & \\
\hline H14A & 0.9130 & 0.6737 & -0.3085 & $0.042^{\star}$ & \\
\hline H14B & 0.8941 & 0.7377 & -0.2422 & $0.042^{\star}$ & \\
\hline H14C & 0.8322 & 0.7041 & -0.3066 & $0.042^{\star}$ & \\
\hline C15 & $0.8541(2)$ & $0.4003(2)$ & $0.0034(3)$ & $0.0159(9)$ & \\
\hline C16 & $0.8354(3)$ & $0.3286(2)$ & $-0.0059(3)$ & $0.0197(10)$ & \\
\hline H16 & 0.7860 & 0.3157 & -0.0142 & $0.024^{\star}$ & \\
\hline C17 & $0.8879(3)$ & $0.2756(2)$ & $-0.0031(3)$ & $0.0240(11)$ & \\
\hline H17 & 0.8738 & 0.2271 & -0.0101 & $0.029^{\star}$ & \\
\hline C18 & $0.9605(3)$ & $0.2921(2)$ & $0.0097(3)$ & $0.0216(10)$ & \\
\hline C19 & $0.9795(3)$ & $0.3640(3)$ & $0.0204(3)$ & $0.0219(10)$ & \\
\hline H19 & 1.0290 & 0.3767 & 0.0288 & $0.026^{\star}$ & \\
\hline C20 & $0.9271(2)$ & $0.4168(2)$ & $0.0189(3)$ & $0.0201(10)$ & \\
\hline
\end{tabular}




\begin{tabular}{|c|c|c|c|c|}
\hline $\mathrm{H} 2 \mathrm{O}$ & 0.9410 & 0.4651 & 0.0286 & $0.024^{*}$ \\
\hline C21 & $1.0178(3)$ & $0.2344(3)$ & $0.0113(4)$ & $0.0348(13)$ \\
\hline $\mathrm{H} 21 \mathrm{~A}$ & 1.0485 & 0.2400 & -0.0406 & $0.052^{*}$ \\
\hline H21B & 0.9945 & 0.1875 & 0.0090 & $0.052^{*}$ \\
\hline $\mathrm{H} 21 \mathrm{C}$ & 1.0476 & 0.2384 & 0.0666 & $0.052^{*}$ \\
\hline C22 & $0.8191(2)$ & $0.4573(2)$ & $0.5615(3)$ & $0.0167(9)$ \\
\hline C23 & $0.8132(2)$ & $0.4047(2)$ & $0.6263(3)$ & $0.0206(10)$ \\
\hline H23 & 0.7676 & 0.3829 & 0.6351 & $0.025^{\star}$ \\
\hline C24 & $0.8732(2)$ & $0.3834(2)$ & $0.6785(3)$ & $0.0213(10)$ \\
\hline H24 & 0.8681 & 0.3469 & 0.7220 & $0.026^{*}$ \\
\hline C25 & $0.9404(2)$ & $0.4152(2)$ & $0.6676(3)$ & $0.0224(10)$ \\
\hline C26 & $0.9467(3)$ & $0.4665(2)$ & $0.6006(3)$ & $0.0237(11)$ \\
\hline H26 & 0.9925 & 0.4874 & 0.5904 & $0.028^{*}$ \\
\hline C27 & $0.8867(2)$ & $0.4875(2)$ & $0.5484(3)$ & $0.0200(10)$ \\
\hline $\mathrm{H} 27$ & 0.8920 & 0.5228 & 0.5034 & $0.024^{*}$ \\
\hline C28 & $1.0047(3)$ & $0.3951(3)$ & $0.7271(3)$ & $0.0312(12)$ \\
\hline $\mathrm{H} 28 \mathrm{~A}$ & 0.9886 & 0.3856 & 0.7879 & $0.047^{*}$ \\
\hline H28B & 1.0396 & 0.4345 & 0.7288 & $0.047^{*}$ \\
\hline $\mathrm{H} 28 \mathrm{C}$ & 1.0277 & 0.3523 & 0.7034 & $0.047^{*}$ \\
\hline C29 & $0.7175(2)$ & $0.5702(2)$ & $0.5717(3)$ & $0.0166(9)$ \\
\hline C30 & $0.7101(2)$ & $0.5582(3)$ & $0.6632(3)$ & $0.0205(10)$ \\
\hline H3O & 0.7193 & 0.5119 & 0.6870 & $0.025^{\star}$ \\
\hline C31 & $0.6898(2)$ & $0.6121(2)$ & $0.7198(3)$ & $0.0216(10)$ \\
\hline H31 & 0.6835 & 0.6021 & 0.7815 & $0.026^{*}$ \\
\hline C32 & $0.6784(3)$ & $0.6816(3)$ & 0.6875 & $0.0242(11)$ \\
\hline
\end{tabular}




\begin{tabular}{|l|l|l|l|l|l|}
\hline C33 & $0.6863(3)$ & $0.6937(2)$ & $0.5969(3)$ & $0.0247(11)$ & \\
\hline H33 & 0.6786 & 0.7403 & 0.5735 & $0.030^{\star}$ & \\
\hline C34 & $0.7056(3)$ & $0.6388(2)$ & $0.5392(3)$ & $0.0218(10)$ & \\
\hline H34 & 0.7105 & 0.6483 & 0.4771 & $0.026^{\star}$ & \\
\hline C35 & $0.6576(3)$ & $0.7410(3)$ & $0.7489(4)$ & $0.0358(14)$ & \\
\hline H35A & 0.6086 & 0.7327 & 0.7698 & $0.054^{\star}$ & \\
\hline H35B & 0.6589 & 0.7865 & 0.7166 & $0.054^{\star}$ & \\
\hline H35C & 0.6916 & 0.7427 & 0.8005 & $0.054^{\star}$ & \\
\hline C36 & $0.6674(2)$ & $0.4326(2)$ & $0.5165(3)$ & $0.0182(10)$ & \\
\hline C37 & $0.6754(3)$ & $0.3610(2)$ & $0.4919(3)$ & $0.0239(11)$ & \\
\hline H37 & 0.7210 & 0.3440 & 0.4733 & $0.029^{\star}$ & \\
\hline C38 & $0.6167(3)$ & $0.3149(3)$ & $0.4947(3)$ & $0.0277(12)$ & \\
\hline H38 & 0.6222 & 0.2669 & 0.4757 & $0.033^{\star}$ & \\
\hline C39 & $0.5499(3)$ & $0.3375(3)$ & $0.5249(3)$ & $0.0253(11)$ & \\
\hline C40 & $0.5429(3)$ & $0.4081(3)$ & $0.5487(3)$ & $0.0235(11)$ & \\
\hline H40 & 0.4977 & 0.4247 & 0.5689 & $0.028^{\star}$ & \\
\hline C41 & $0.6002(2)$ & $0.4555(3)$ & $0.5438(3)$ & $0.0216(10)$ & \\
\hline H41 & 0.5934 & 0.5042 & 0.5592 & $0.026^{\star}$ & \\
\hline C42 & $0.4871(3)$ & $0.2861(3)$ & $0.5288(4)$ & $0.0396(14)$ & \\
\hline H42A & 0.4449 & 0.3108 & 0.5520 & $0.059^{\star}$ & \\
\hline H42B & 0.5002 & 0.2461 & 0.5683 & $0.059^{\star}$ & \\
\hline H42C & 0.4753 & 0.2680 & 0.4684 & $0.059^{\star}$ & \\
\hline C43 & $0.7013(2)$ & $0.3863(2)$ & $0.2485(3)$ & $0.0200(10)$ & \\
\hline C44 & $0.6742(3)$ & $0.3105(3)$ & $0.2501(4)$ & $0.0333(13)$ & \\
\hline H44A & 0.7102 & 0.2812 & 0.2796 & $0.040^{\star}$ & 0.73 \\
\hline & & & & & \\
\hline
\end{tabular}




\begin{tabular}{|l|l|l|l|l|l|}
\hline H44B & 0.6317 & 0.3090 & 0.2855 & $0.040^{\star}$ & 0.73 \\
\hline H44C & 0.6244 & 0.3098 & 0.2298 & $0.040^{\star}$ & 0.27 \\
\hline H44D & 0.6764 & 0.2933 & 0.3108 & $0.040^{\star}$ & 0.27 \\
\hline C45 & $0.6568(6)$ & $0.2799(4)$ & $0.1632(5)$ & $0.050(2)$ & 0.73 \\
\hline H45A & 0.6211 & 0.3101 & 0.1314 & $0.074^{\star}$ & 0.73 \\
\hline H45B & 0.6368 & 0.2320 & 0.1708 & $0.074^{\star}$ & 0.73 \\
\hline H45C & 0.7007 & 0.2770 & 0.1284 & $0.074^{\star}$ & 0.73 \\
\hline C45A & $0.7161(12)$ & $0.2623(7)$ & $0.1944(17)$ & $0.053(6)$ & 0.27 \\
\hline H45D & 0.7320 & 0.2880 & 0.1414 & $0.079^{\star}$ & 0.27 \\
\hline H45E & 0.6859 & 0.2217 & 0.1756 & $0.079^{\star}$ & 0.27 \\
\hline H45F & 0.7585 & 0.2450 & 0.2291 & $0.079^{\star}$ & 0.27 \\
\hline C46 & $0.8792(2)$ & $0.4331(2)$ & $0.2770(3)$ & $0.0152(9)$ & \\
\hline C47 & $0.9384(2)$ & $0.3784(2)$ & $0.2877(3)$ & $0.0211(10)$ & \\
\hline H47A & 0.9586 & 0.3797 & 0.3499 & $0.025^{\star}$ & \\
\hline H47B & 0.9778 & 0.3897 & 0.2467 & $0.025^{\star}$ & \\
\hline C48 & $0.9092(3)$ & $0.3045(3)$ & $0.2671(4)$ & $0.0360(13)$ & \\
\hline H48A & 0.8704 & 0.2933 & 0.3080 & $0.054^{\star}$ & \\
\hline H48B & 0.9482 & 0.2694 & 0.2748 & $0.054^{\star}$ & \\
\hline H48C & 0.8901 & 0.3031 & 0.2051 & $0.054^{\star}$ & \\
\hline C49 & $0.8253(3)$ & $0.5936(3)$ & $0.1509(3)$ & $0.0203(10)$ & \\
\hline C50 & $0.6878(3)$ & $0.5657(2)$ & $0.1278(3)$ & $0.0195(10)$ & \\
\hline C51 & $0.8005(3)$ & $0.6073(2)$ & $0.3551(3)$ & $0.0194(10)$ & \\
\hline C52 & $0.6668(3)$ & $0.5639(2)$ & $0.3303(3)$ & $0.0177(10)$ & \\
\hline C53 & $0.5166(3)$ & $0.9665(3)$ & $0.2748(4)$ & $0.0437(15)$ & \\
\hline H53A & 0.4810 & 1.0036 & 0.2903 & $0.052^{\star}$ & \\
\hline & & & & & \\
\hline
\end{tabular}




\begin{tabular}{|l|l|l|l|l|l|}
\hline H53B & 0.5619 & 0.9909 & 0.2588 & $0.052^{*}$ & \\
\hline O1 & $\begin{array}{l}0.71078 \\
(19)\end{array}$ & $\begin{array}{l}0.41452 \\
(16)\end{array}$ & $0.1737(2)$ & $0.0250(8)$ & \\
\hline O2 & $\begin{array}{l}0.71458 \\
(19)\end{array}$ & $\begin{array}{l}0.41517 \\
(17)\end{array}$ & $0.3238(2)$ & $0.0257(8)$ & \\
\hline O3 & $\begin{array}{l}0.85738 \\
(17)\end{array}$ & $\begin{array}{l}0.44616 \\
(16)\end{array}$ & $0.1974(2)$ & $0.0215(7)$ & \\
\hline O4 & $\begin{array}{l}0.85480 \\
(16)\end{array}$ & $\begin{array}{l}0.46159 \\
(16)\end{array}$ & $0.3465(2)$ & $0.0199(7)$ & \\
\hline O5 & $\begin{array}{l}0.86026 \\
(19)\end{array}$ & $\begin{array}{l}0.64365 \\
(18)\end{array}$ & $0.1453(2)$ & $0.0301(8)$ & \\
\hline O6 & $\begin{array}{l}0.63722 \\
(19)\end{array}$ & $0.5994(2)$ & $0.1074(2)$ & $0.0362(9)$ & \\
\hline O7 & $\begin{array}{l}0.83055 \\
(18)\end{array}$ & $\begin{array}{l}0.66144 \\
(17)\end{array}$ & $0.3629(2)$ & $0.0278(8)$ & \\
\hline O8 & $\begin{array}{l}0.61028 \\
(17)\end{array}$ & $\begin{array}{l}0.59124 \\
(18)\end{array}$ & $0.3218(2)$ & $0.0266(8)$ & \\
\hline Os1 & $0.76852(2)$ & $0.51169(2)$ & $0.15852(2)$ & $0.01284(5)$ & \\
\hline Os2 & $0.75547(2)$ & $0.51941(2)$ & $0.34254(2)$ & $0.01333(5)$ & \\
\hline P1 & $0.78714(6)$ & $0.47126(6)$ & $0.00812(8)$ & $0.0143(2)$ & \\
\hline P2 & $0.74144(6)$ & $0.49529(6)$ & $0.49914(8)$ & $0.0156(2)$ & \\
\hline $\begin{array}{l}(11) \\
\text { Cl1 }\end{array}$ & $\begin{array}{l}0.53392 \\
(11)\end{array}$ & 0.91213 & $\begin{array}{l}0.36866 \\
(11)\end{array}$ & $0.0674(5)$ & \\
\hline
\end{tabular}

Atomic displacement parameters $\left(\AA^{2}\right)$ for $(7) \cdot \mathrm{CH}_{2} \mathrm{Cl}_{2}$

\begin{tabular}{|l|l|l|l|l|l|l|}
\hline & $U^{11}$ & $U^{22}$ & $U^{\beta 3}$ & $U^{12}$ & $U^{13}$ & $U^{23}$ \\
\hline
\end{tabular}




\begin{tabular}{|c|c|c|c|c|c|c|}
\hline C1 & $0.016(2)$ & $0.012(2)$ & $0.016(2)$ & $\begin{array}{l}0.0012 \\
(16)\end{array}$ & $\begin{array}{l}-0.0008 \\
(18)\end{array}$ & $\begin{array}{l}-0.0003 \\
(17)\end{array}$ \\
\hline C2 & $0.019(3)$ & $0.029(3)$ & $0.017(2)$ & $\begin{array}{l}0.0015 \\
(19)\end{array}$ & $\begin{array}{l}0.0021 \\
(19)\end{array}$ & $-0.003(2)$ \\
\hline C3 & 0.018 (3) & $0.032(3)$ & $0.025(3)$ & $-0.002(2)$ & $0.004(2)$ & $-0.003(2)$ \\
\hline C4 & $0.018(3)$ & $0.019(2)$ & $0.023(3)$ & $\begin{array}{l}-0.0001 \\
(18)\end{array}$ & $-0.004(2)$ & $0.001(2)$ \\
\hline $\mathrm{C} 5$ & $0.024(3)$ & $0.034(3)$ & $0.016(2)$ & $-0.001(2)$ & $-0.002(2)$ & $-0.005(2)$ \\
\hline C6 & $0.023(3)$ & $0.026(3)$ & $0.016(2)$ & $\begin{array}{l}-0.0069 \\
(19)\end{array}$ & $0.003(2)$ & $-0.002(2)$ \\
\hline $\mathrm{C7}$ & $0.016(3)$ & $0.032(3)$ & $0.023(3)$ & $-0.001(2)$ & $0.000(2)$ & $-0.006(2)$ \\
\hline C8 & $0.017(2)$ & $0.017(2)$ & $0.012(2)$ & $\begin{array}{l}-0.0020 \\
(17)\end{array}$ & $\begin{array}{l}-0.0046 \\
(17)\end{array}$ & $\begin{array}{l}-0.0024 \\
(18)\end{array}$ \\
\hline C9 & $0.016(2)$ & $0.017(2)$ & $0.021(2)$ & $\begin{array}{l}-0.0023 \\
(18)\end{array}$ & $\begin{array}{l}0.0004 \\
(18)\end{array}$ & $\begin{array}{l}0.0006 \\
(19)\end{array}$ \\
\hline C10 & $0.022(3)$ & $0.023(2)$ & $0.012(2)$ & $\begin{array}{l}-0.0041 \\
(19)\end{array}$ & $\begin{array}{l}0.0058 \\
(19)\end{array}$ & $\begin{array}{l}-0.0026 \\
(19)\end{array}$ \\
\hline C11 & $0.014(2)$ & $0.022(2)$ & $0.020(2)$ & $\begin{array}{l}-0.0040 \\
(18)\end{array}$ & $\begin{array}{l}-0.0023 \\
(19)\end{array}$ & $0.007(2)$ \\
\hline C12 & $0.015(2)$ & $0.018(2)$ & $0.020(2)$ & $\begin{array}{l}0.0005 \\
(17)\end{array}$ & $\begin{array}{l}-0.0042 \\
(19)\end{array}$ & $\begin{array}{l}-0.0013 \\
(19)\end{array}$ \\
\hline $\mathrm{C} 13$ & $0.016(2)$ & $0.021(2)$ & 0.011 (2) & $\begin{array}{l}-0.0004 \\
(18)\end{array}$ & $\begin{array}{l}0.0025 \\
(18)\end{array}$ & $\begin{array}{l}-0.0006 \\
(18)\end{array}$ \\
\hline C14 & $0.030(3)$ & 0.027 (3) & 0.028 (3) & $0.003(2)$ & $0.006(2)$ & $0.011(2)$ \\
\hline C15 & $0.020(2)$ & $0.020(2)$ & $0.008(2)$ & $\begin{array}{l}-0.0017 \\
(18)\end{array}$ & $\begin{array}{l}0.0016 \\
(18)\end{array}$ & $\begin{array}{l}0.0016 \\
(18)\end{array}$ \\
\hline C16 & $0.022(3)$ & $0.023(2)$ & 0.014 (2) & $\begin{array}{l}-0.0025 \\
(19)\end{array}$ & $\begin{array}{l}0.0003 \\
(19)\end{array}$ & $\begin{array}{l}-0.0002 \\
(19)\end{array}$ \\
\hline
\end{tabular}




\begin{tabular}{|l|l|l|l|l|l|l|}
\hline C17 & $0.033(3)$ & $0.017(2)$ & $0.022(3)$ & $0.002(2)$ & $0.000(2)$ & $-0.001(2)$ \\
\hline C18 & $0.026(3)$ & $0.025(3)$ & $0.014(2)$ & $0.009(2)$ & $0.000(2)$ & $0.000(2)$ \\
\hline C19 & $0.017(3)$ & $0.033(3)$ & $0.015(2)$ & $0.002(2)$ & $\begin{array}{l}0.0001 \\
(19)\end{array}$ & $-0.001(2)$ \\
\hline C20 & $0.019(3)$ & $0.023(2)$ & $0.019(2)$ & $\begin{array}{l}-0.0007 \\
(19)\end{array}$ & $\begin{array}{l}0.0002 \\
(19)\end{array}$ & $-0.001(2)$ \\
\hline C21 & $0.035(3)$ & $0.033(3)$ & $0.036(3)$ & $0.015(2)$ & $0.001(3)$ & $-0.003(3)$ \\
\hline C22 & $0.020(2)$ & $0.016(2)$ & $0.014(2)$ & $\begin{array}{l}0.0049 \\
(18)\end{array}$ & $\begin{array}{l}0.0011 \\
(18)\end{array}$ & 0.0007 \\
\hline C23 & $0.016(2)$ & $0.021(2)$ & $0.026(3)$ & $\begin{array}{l}0.0002 \\
(18)\end{array}$ & $0.003(2)$ & $0.001(2)$ \\
\hline C24 & $0.021(3)$ & $0.022(2)$ & $0.021(3)$ & $\begin{array}{l}0.0046 \\
(19)\end{array}$ & $0.002(2)$ & $0.002(2)$ \\
\hline C25 & $0.019(3)$ & $0.025(3)$ & $0.022(3)$ & $\begin{array}{l}0.0061 \\
(19)\end{array}$ & $-0.001(2)$ & $-0.006(2)$ \\
\hline C26 & $0.020(3)$ & $0.028(3)$ & $0.024(3)$ & $\begin{array}{l}0.0023 \\
(19)\end{array}$ & $0.006(2)$ & $-0.006(2)$ \\
\hline C27 & $0.023(3)$ & $0.024(2)$ & $0.013(2)$ & $\begin{array}{l}0.0016 \\
(19)\end{array}$ & $\begin{array}{l}0.0044 \\
(19)\end{array}$ & $\begin{array}{l}-0.0013 \\
(19)\end{array}$ \\
\hline C28 & $0.030(3)$ & $0.035(3)$ & $0.028(3)$ & $0.005(2)$ & $-0.006(2)$ & $0.003(2)$ \\
\hline C29 & $0.014(2)$ & $0.021(2)$ & $0.015(2)$ & $\begin{array}{l}0.0025 \\
(17)\end{array}$ & $\begin{array}{l}0.0036 \\
(18)\end{array}$ & -0.0048 \\
$(19)$
\end{tabular}




\begin{tabular}{|c|c|c|c|c|c|c|}
\hline C34 & $0.025(3)$ & $0.021(2)$ & $0.019(3)$ & $\begin{array}{l}0.0049 \\
(19)\end{array}$ & $0.001(2)$ & $0.001(2)$ \\
\hline C35 & 0.041 (3) & $0.038(3)$ & $0.028(3)$ & $0.016(3)$ & $-0.004(3)$ & $-0.013(2)$ \\
\hline C36 & $0.022(3)$ & $0.019(2)$ & $0.014(2)$ & $\begin{array}{l}0.0020 \\
(18)\end{array}$ & $\begin{array}{l}0.0030 \\
(19)\end{array}$ & $\begin{array}{l}0.0047 \\
(19)\end{array}$ \\
\hline C37 & $0.024(3)$ & $0.022(2)$ & $0.027(3)$ & $\begin{array}{l}0.0011 \\
(19)\end{array}$ & $0.010(2)$ & $0.005(2)$ \\
\hline C38 & $0.030(3)$ & $0.021(2)$ & $0.032(3)$ & $-0.004(2)$ & $0.008(2)$ & $0.000(2)$ \\
\hline C39 & $0.023(3)$ & $0.027(3)$ & $0.026(3)$ & $-0.005(2)$ & $0.000(2)$ & 0.005 (2) \\
\hline $\mathrm{C} 40$ & $0.016(3)$ & 0.035 (3) & $0.020(3)$ & $0.001(2)$ & $0.003(2)$ & $-0.001(2)$ \\
\hline C41 & $0.023(3)$ & $0.021(2)$ & $0.021(3)$ & $\begin{array}{l}0.0030 \\
(19)\end{array}$ & $0.000(2)$ & $0.005(2)$ \\
\hline C42 & $0.032(3)$ & $0.037(3)$ & $0.051(4)$ & $-0.011(2)$ & $0.010(3)$ & $-0.001(3)$ \\
\hline $\mathrm{C} 43$ & $0.018(3)$ & $0.014(2)$ & $0.028(3)$ & $\begin{array}{l}-0.0045 \\
(18)\end{array}$ & $0.008(2)$ & $0.001(2)$ \\
\hline C44 & $0.043(3)$ & $0.020(3)$ & $0.037(3)$ & $-0.010(2)$ & $0.011(3)$ & $-0.005(2)$ \\
\hline $\mathrm{C} 45$ & $0.092(7)$ & $0.030(4)$ & $0.027(4)$ & $-0.026(4)$ & $-0.007(4)$ & $0.006(3)$ \\
\hline C45A & $0.066(12)$ & 0.029 (9) & $0.064(12)$ & $-0.009(9)$ & $0.018(10)$ & $-0.005(9)$ \\
\hline C46 & $0.017(2)$ & $0.012(2)$ & $0.017(2)$ & $\begin{array}{l}-0.0012 \\
(16)\end{array}$ & $\begin{array}{l}0.0025 \\
(18)\end{array}$ & $\begin{array}{l}-0.0002 \\
(18)\end{array}$ \\
\hline C47 & $0.020(3)$ & $0.025(3)$ & $0.019(2)$ & $\begin{array}{l}0.0062 \\
(19)\end{array}$ & $-0.001(2)$ & $0.001(2)$ \\
\hline C48 & $0.042(3)$ & $0.023(3)$ & $0.042(3)$ & $0.001(2)$ & $-0.010(3)$ & 0.001 (2) \\
\hline C49 & $0.021(3)$ & $0.028(3)$ & $0.012(2)$ & $-0.007(2)$ & $\begin{array}{l}0.0004 \\
(19)\end{array}$ & $-0.002(2)$ \\
\hline C50 & $0.021(3)$ & $0.028(3)$ & $0.010(2)$ & $-0.004(2)$ & $\begin{array}{l}0.0054 \\
(19)\end{array}$ & $\begin{array}{l}-0.0076 \\
(19)\end{array}$ \\
\hline C51 & $0.027(3)$ & $0.022(2)$ & $0.009(2)$ & $-0.001(2)$ & 0.0046 & -0.0026 \\
\hline
\end{tabular}




\begin{tabular}{|l|l|l|l|l|l|l|}
\hline & & & & & $(19)$ & $(19)$ \\
\hline C52 & $0.028(3)$ & $0.017(2)$ & $0.009(2)$ & $\begin{array}{l}-0.0085 \\
(19)\end{array}$ & $\begin{array}{l}0.0048 \\
(19)\end{array}$ & -0.0014 \\
$(18)$
\end{tabular}




\begin{tabular}{|l|l|l|l|l|l|l|}
\hline Cl2 & $\begin{array}{l}0.0841 \\
(14)\end{array}$ & $\begin{array}{l}0.0830 \\
(13)\end{array}$ & $0.0365(9)$ & $\begin{array}{l}0.0053 \\
(10)\end{array}$ & $0.0230(9)$ & $0.0165(9)$ \\
\hline
\end{tabular}

Geometric parameters (Å, ㅇ) for (7) $\cdot \mathrm{CH}_{2} \mathrm{Cl}_{2}$

\begin{tabular}{|l|l|l|l|}
\hline C1-C6 & $1.387(6)$ & C32-C33 & $1.383(7)$ \\
\hline C1-C2 & $1.393(6)$ & C32-C35 & $1.500(6)$ \\
\hline C1-P1 & $1.838(4)$ & C33-C34 & $1.395(6)$ \\
\hline C2-C3 & $1.395(6)$ & C33-H33 & 0.9500 \\
\hline C2-H2 & 0.9500 & C34-H34 & 0.9500 \\
\hline C3-C4 & $1.390(7)$ & C35-H35A & 0.9800 \\
\hline C3-H3 & 0.9500 & C35-H35B & 0.9800 \\
\hline C4-C5 & $1.388(7)$ & C35-H35C & 0.9800 \\
\hline C4-C7 & $1.505(6)$ & C36-C41 & $1.388(6)$ \\
\hline C5-C6 & $1.394(6)$ & C36-C37 & $1.400(6)$ \\
\hline C5-H5 & 0.9500 & C36-P2 & $1.829(5)$ \\
\hline C6-H6 & 0.9500 & C37-C38 & $1.390(6)$ \\
\hline C7-H7A & 0.9800 & C37-H37 & 0.9500 \\
\hline C7-H7B & 0.9800 & C38-C39 & $1.394(7)$ \\
\hline C7-H7C & 0.9800 & C38-H38 & 0.9500 \\
\hline C8-C9 & $1.394(6)$ & C39-C40 & $1.376(7)$ \\
\hline C8-C13 & $1.399(6)$ & C39-C42 & $1.512(7)$ \\
\hline C8-P1 & $1.816(4)$ & C40-C41 & $1.388(6)$ \\
\hline C9-C10 & $1.400(6)$ & C40-H40 & 0.9500 \\
\hline C9-H9 & 0.9500 & C41-H41 & 0.9500 \\
\hline C10-C11 & $1.386(6)$ & C42-H42A & 0.9800 \\
\hline C10-H10 & 0.9500 & C42-H42B & 0.9800 \\
\hline
\end{tabular}




\begin{tabular}{|l|l|l|l|}
\hline C11-C12 & $1.388(6)$ & C42-H42C & 0.9800 \\
\hline C11-C14 & $1.498(6)$ & C43-O1 & $1.252(5)$ \\
\hline C12-C13 & $1.381(6)$ & C43-O2 & $1.263(6)$ \\
\hline C12-H12 & 0.9500 & C43-C44 & $1.507(6)$ \\
\hline C13-H13 & 0.9500 & C44-C45 & $1.445(8)$ \\
\hline C14-H14A & 0.9800 & C44-C45A & $1.466(11)$ \\
\hline C14-H14B & 0.9800 & C44-H44A & 0.9600 \\
\hline C14-H14C & 0.9800 & C44-H44B & 0.9600 \\
\hline C15-C16 & $1.393(6)$ & C44-H44C & 0.9601 \\
\hline C15-C20 & $1.398(6)$ & C44-H44D & 0.9599 \\
\hline C15-P1 & $1.820(4)$ & C45-H45A & 0.9800 \\
\hline C16-C17 & $1.388(6)$ & C45-H45B & 0.9800 \\
\hline C16-H16 & 0.9500 & C45-H45C & 0.9800 \\
\hline C17-C18 & $1.385(7)$ & C45A-H45D & 0.9800 \\
\hline C17-H17 & 0.9500 & C45A-H45E & 0.9800 \\
\hline C18-C19 & $1.400(7)$ & C45A-H45F & 0.9800 \\
\hline C18-C21 & $1.514(6)$ & C46-O4 & $1.262(5)$ \\
\hline C19-C20 & $1.385(6)$ & C46-O3 & $1.267(5)$ \\
\hline C19-H19 & 0.9500 & C46-C47 & $1.505(6)$ \\
\hline C20-H20 & 0.9500 & C47-C48 & $1.513(7)$ \\
\hline C21-H21A & 0.9800 & C47-H47A & 0.9900 \\
\hline C21-H21B & 0.9800 & C47-H47B & 0.9900 \\
\hline C21-H21C & 0.9800 & C48-H48A & 0.9800 \\
\hline C22-C23 & $1.388(6)$ & C48-H48B & 0.9800 \\
\hline C22-C27 & $1.392(6)$ & C48-H48C & 0.9800 \\
\hline
\end{tabular}




\begin{tabular}{|c|c|c|c|}
\hline C22-P2 & $1.831(4)$ & $\mathrm{C} 49-\mathrm{O} 5$ & $1.143(5)$ \\
\hline C23-C24 & $1.394(6)$ & C49-Os1 & $1.866(5)$ \\
\hline $\mathrm{C} 23-\mathrm{H} 23$ & 0.9500 & $\mathrm{C} 50-\mathrm{O} 6$ & $1.162(5)$ \\
\hline C24-C25 & $1.394(7)$ & C50-Os1 & $1.849(5)$ \\
\hline $\mathrm{C} 24-\mathrm{H} 24$ & 0.9500 & $\mathrm{C} 51-\mathrm{O} 7$ & $1.161(5)$ \\
\hline C25-C26 & $1.395(7)$ & C51-Os2 & $1.853(5)$ \\
\hline C25-C28 & $1.509(6)$ & C52-O8 & $1.168(5)$ \\
\hline C26-C27 & $1.392(6)$ & C52-Os2 & $1.843(5)$ \\
\hline C26-H26 & 0.9500 & $\mathrm{C} 53-\mathrm{Cl} 1$ & $1.751(6)$ \\
\hline $\mathrm{C} 27-\mathrm{H} 27$ & 0.9500 & $\mathrm{C} 53-\mathrm{Cl} 2$ & $1.753(6)$ \\
\hline $\mathrm{C} 28-\mathrm{H} 28 \mathrm{~A}$ & 0.9800 & C53-H53A & 0.9900 \\
\hline $\mathrm{C} 28-\mathrm{H} 28 \mathrm{~B}$ & 0.9800 & C53-H53B & 0.9900 \\
\hline $\mathrm{C} 28-\mathrm{H} 28 \mathrm{C}$ & 0.9800 & O1-Os1 & 2.127 (3) \\
\hline C29-C34 & $1.389(6)$ & O2-Os2 & $2.110(3)$ \\
\hline C29-C30 & $1.395(6)$ & O3-Os1 & $2.118(3)$ \\
\hline C29-P2 & $1.836(4)$ & O4-Os2 & $2.132(3)$ \\
\hline C30-C31 & $1.377(6)$ & Os1-P1 & $2.4037(11)$ \\
\hline $\mathrm{C} 30-\mathrm{H} 30$ & 0.9500 & Os1-Os2 & 2.7677 (3) \\
\hline C31-C32 & $1.403(7)$ & Os2-P2 & $2.4022(12)$ \\
\hline $\mathrm{C} 31-\mathrm{H} 31$ & 0.9500 & & \\
\hline $\mathrm{C} 6-\mathrm{C} 1-\mathrm{C} 2$ & $118.1(4)$ & $\mathrm{C} 41-\mathrm{C} 36-\mathrm{P} 2$ & $121.7(3)$ \\
\hline $\mathrm{C} 6-\mathrm{C} 1-\mathrm{P} 1$ & $120.6(3)$ & $\mathrm{C} 37-\mathrm{C} 36-\mathrm{P} 2$ & $119.5(3)$ \\
\hline $\mathrm{C} 2-\mathrm{C} 1-\mathrm{P} 1$ & $121.3(3)$ & C38-C37-C36 & $119.9(4)$ \\
\hline $\mathrm{C} 1-\mathrm{C} 2-\mathrm{C} 3$ & $120.8(4)$ & $\mathrm{C} 38-\mathrm{C} 37-\mathrm{H} 37$ & 120.1 \\
\hline
\end{tabular}




\begin{tabular}{|l|l|l|l|}
\hline C1-C2-H2 & 119.6 & C36-C37-H37 & 120.1 \\
\hline C3-C2-H2 & 119.6 & C37-C38-C39 & $121.5(5)$ \\
\hline C4-C3-C2 & $121.3(4)$ & C37-C38-H38 & 119.3 \\
\hline C4-C3-H3 & 119.4 & C39-C38-H38 & 119.3 \\
\hline C2-C3-H3 & 119.4 & C40-C39-C38 & $117.8(4)$ \\
\hline C5-C4-C3 & $117.4(4)$ & C40-C39-C42 & $121.5(5)$ \\
\hline C5-C4-C7 & $121.4(4)$ & C38-C39-C42 & $120.6(5)$ \\
\hline C3-C4-C7 & $121.2(4)$ & C39-C40-C41 & $121.5(4)$ \\
\hline C4-C5-C6 & $121.6(4)$ & C39-C40-H40 & 119.2 \\
\hline C4-C5-H5 & 119.2 & C41-C40-H40 & 119.2 \\
\hline C6-C5-H5 & 119.2 & C40-C41-C36 & $120.7(4)$ \\
\hline C1-C6-C5 & $120.7(4)$ & C40-C41-H41 & 119.6 \\
\hline C1-C6-H6 & 119.6 & C36-C41-H41 & 119.6 \\
\hline C5-C6-H6 & 119.6 & C39-C42-H42A & 109.5 \\
\hline C4-C7-H7A & 109.5 & C39-C42-H42B & 109.5 \\
\hline C4-C7-H7B & 109.5 & $\begin{array}{l}\text { H42A-C42- } \\
\text { H42B }\end{array}$ & 109.5 \\
\hline H7A-C7-H7B & 109.5 & C39-C42-H42C & 109.5 \\
\hline C4-C7-H7C & 109.5 & H42A-C42- & 109.5 \\
\hline H7A-C7-H7C & 109.5 & H42B-C42- & 109.5 \\
\hline H7B-C7-H7C & 109.5 & O42C & $125.7(4)$ \\
\hline C9-C8-C13 & $117.5(4)$ & $122.2(3)$ & O1-C43-O2 \\
\hline C9-C8-P1 & $120.1(3)$ & O2-C43-C44 & $116.4(4)$ \\
\hline C13-C8-P1 & $115.1(5)$ \\
\hline
\end{tabular}




\begin{tabular}{|c|c|c|c|}
\hline $\mathrm{C} 8-\mathrm{C} 9-\mathrm{C} 10$ & $120.4(4)$ & C45A-C44-C43 & $112.9(7)$ \\
\hline $\mathrm{C} 8-\mathrm{C} 9-\mathrm{H} 9$ & 119.8 & $\mathrm{C} 45-\mathrm{C} 44-\mathrm{H} 44 \mathrm{~A}$ & 108.4 \\
\hline $\mathrm{C} 10-\mathrm{C} 9-\mathrm{H} 9$ & 119.8 & $\mathrm{C} 43-\mathrm{C} 44-\mathrm{H} 44 \mathrm{~A}$ & 108.6 \\
\hline $\mathrm{C} 11-\mathrm{C} 10-\mathrm{C} 9$ & $121.8(4)$ & C45-C44-H44B & 108.6 \\
\hline $\mathrm{C} 11-\mathrm{C} 10-\mathrm{H} 10$ & 119.1 & C43-C44-H44B & 108.4 \\
\hline $\mathrm{C} 9-\mathrm{C} 10-\mathrm{H} 10$ & 119.1 & $\begin{array}{l}\mathrm{H} 44 \mathrm{~A}-\mathrm{C} 44- \\
\mathrm{H} 44 \mathrm{~B}\end{array}$ & 107.5 \\
\hline C10-C11-C12 & $117.2(4)$ & $\begin{array}{l}\mathrm{C} 45 \mathrm{~A}-\mathrm{C} 44- \\
\mathrm{H} 44 \mathrm{C}\end{array}$ & 109.4 \\
\hline $\mathrm{C} 10-\mathrm{C} 11-\mathrm{C} 14$ & $120.4(4)$ & $\mathrm{C} 43-\mathrm{C} 44-\mathrm{H} 44 \mathrm{C}$ & 108.9 \\
\hline C12-C11-C14 & $122.3(4)$ & $\begin{array}{l}\text { C45A-C44- } \\
\mathrm{H} 44 \mathrm{D}\end{array}$ & 108.7 \\
\hline $\mathrm{C} 13-\mathrm{C} 12-\mathrm{C} 11$ & $121.7(4)$ & C43-C44-H44D & 109.1 \\
\hline $\mathrm{C} 13-\mathrm{C} 12-\mathrm{H} 12$ & 119.2 & $\begin{array}{l}\mathrm{H} 44 \mathrm{C}-\mathrm{C} 44- \\
\mathrm{H} 44 \mathrm{D}\end{array}$ & 107.8 \\
\hline $\mathrm{C} 11-\mathrm{C} 12-\mathrm{H} 12$ & 119.2 & $\mathrm{C} 44-\mathrm{C} 45-\mathrm{H} 45 \mathrm{~A}$ & 109.5 \\
\hline $\mathrm{C} 12-\mathrm{C} 13-\mathrm{C} 8$ & $121.3(4)$ & C44-C45-H45B & 109.5 \\
\hline $\mathrm{C} 12-\mathrm{C} 13-\mathrm{H} 13$ & 119.4 & $\begin{array}{l}\mathrm{H} 45 \mathrm{~A}-\mathrm{C} 45- \\
\mathrm{H} 45 \mathrm{~B}\end{array}$ & 109.5 \\
\hline $\mathrm{C} 8-\mathrm{C} 13-\mathrm{H} 13$ & 119.4 & $\mathrm{C} 44-\mathrm{C} 45-\mathrm{H} 45 \mathrm{C}$ & 109.5 \\
\hline $\mathrm{C} 11-\mathrm{C} 14-\mathrm{H} 14 \mathrm{~A}$ & 109.5 & $\begin{array}{l}\mathrm{H} 45 \mathrm{~A}-\mathrm{C} 45- \\
\mathrm{H} 45 \mathrm{C}\end{array}$ & 109.5 \\
\hline $\mathrm{C} 11-\mathrm{C} 14-\mathrm{H} 14 \mathrm{~B}$ & 109.5 & $\begin{array}{l}\mathrm{H} 45 \mathrm{~B}-\mathrm{C} 45- \\
\mathrm{H} 45 \mathrm{C}\end{array}$ & 109.5 \\
\hline $\mathrm{H} 14 \mathrm{~A}-\mathrm{C} 14-\mathrm{H} 14 \mathrm{~B}$ & 109.5 & $\begin{array}{l}\text { C44-C45A- } \\
\text { H45D }\end{array}$ & 109.5 \\
\hline $\mathrm{C} 11-\mathrm{C} 14-\mathrm{H} 14 \mathrm{C}$ & 109.5 & $\begin{array}{l}\text { C44-C45A- } \\
\text { H45E }\end{array}$ & 109.5 \\
\hline
\end{tabular}




\begin{tabular}{|l|l|l|l|}
\hline H14A-C14-H14C & 109.5 & $\begin{array}{l}\text { H45D-C45A- } \\
\text { H45E }\end{array}$ & 109.5 \\
\hline H14B-C14-H14C & 109.5 & C44-C45A-H45F & 109.5 \\
\hline C16-C15-C20 & $117.7(4)$ & $\begin{array}{l}\text { H45D-C45A- } \\
\text { H45F }\end{array}$ & 109.5 \\
\hline C16-C15-P1 & $122.8(3)$ & $\begin{array}{l}\text { H45E-C45A- } \\
\text { H45F }\end{array}$ & 109.5 \\
\hline C20-C15-P1 & $119.1(3)$ & O4-C46-O3 & $125.3(4)$ \\
\hline C17-C16-C15 & $121.1(4)$ & O4-C46-C47 & $118.6(4)$ \\
\hline C17-C16-H16 & 119.5 & O3-C46-C47 & $116.1(4)$ \\
\hline C15-C16-H16 & 119.5 & C46-C47-C48 & $110.3(4)$ \\
\hline C18-C17-C16 & $121.2(4)$ & C46-C47-H47A & 109.6 \\
\hline C18-C17-H17 & 119.4 & C48-C47-H47A & 109.6 \\
\hline C16-C17-H17 & 119.4 & C46-C47-H47B & 109.6 \\
\hline C17-C18-C19 & $118.0(4)$ & C48-C47-H47B & 109.6 \\
\hline C17-C18-C21 & $121.2(4)$ & $\begin{array}{l}\text { H47A-C47- } \\
\text { H47B }\end{array}$ & 108.1 \\
\hline C19-C18-C21 & $120.8(4)$ & C47-C48-H48A & 109.5 \\
\hline C20-C19-C18 & $120.9(4)$ & C47-C48-H48B & 109.5 \\
\hline C20-C19-H19 & 119.6 & $\begin{array}{l}\text { H48A-C48- } \\
\text { H48B }\end{array}$ & 109.5 \\
\hline C18-C19-H19 & 119.6 & C47-C48-H48C & 109.5 \\
\hline C19-C20-C15 & $121.0(4)$ & $\begin{array}{l}\text { H48A-C48- } \\
\text { H48C }\end{array}$ & 109.5 \\
\hline C15-C20-H20 & 119.5 & H48B-C48- & 109.5 \\
\hline
\end{tabular}




\begin{tabular}{|c|c|c|c|}
\hline $\mathrm{C} 18-\mathrm{C} 21-\mathrm{H} 21 \mathrm{~A}$ & 109.5 & O6-C50-Os 1 & $179.2(4)$ \\
\hline $\mathrm{C} 18-\mathrm{C} 21-\mathrm{H} 21 \mathrm{~B}$ & 109.5 & $\mathrm{O} 7-\mathrm{C} 51-\mathrm{Os} 2$ & $178.1(4)$ \\
\hline $\mathrm{H} 21 \mathrm{~A}-\mathrm{C} 21-\mathrm{H} 21 \mathrm{~B}$ & 109.5 & $\mathrm{O} 8-\mathrm{C} 52-\mathrm{Os} 2$ & $179.0(4)$ \\
\hline $\mathrm{C} 18-\mathrm{C} 21-\mathrm{H} 21 \mathrm{C}$ & 109.5 & $\mathrm{Cl} 1-\mathrm{C} 53-\mathrm{Cl} 2$ & $112.5(3)$ \\
\hline $\mathrm{H} 21 \mathrm{~A}-\mathrm{C} 21-\mathrm{H} 21 \mathrm{C}$ & 109.5 & $\mathrm{Cl} 1-\mathrm{C} 53-\mathrm{H} 53 \mathrm{~A}$ & 109.1 \\
\hline $\mathrm{H} 21 \mathrm{~B}-\mathrm{C} 21-\mathrm{H} 21 \mathrm{C}$ & 109.5 & $\mathrm{Cl} 2-\mathrm{C} 53-\mathrm{H} 53 \mathrm{~A}$ & 109.1 \\
\hline $\mathrm{C} 23-\mathrm{C} 22-\mathrm{C} 27$ & $118.5(4)$ & $\mathrm{Cl} 1-\mathrm{C} 53-\mathrm{H} 53 \mathrm{~B}$ & 109.1 \\
\hline $\mathrm{C} 23-\mathrm{C} 22-\mathrm{P} 2$ & $123.5(3)$ & $\mathrm{Cl} 2-\mathrm{C} 53-\mathrm{H} 53 \mathrm{~B}$ & 109.1 \\
\hline $\mathrm{C} 27-\mathrm{C} 22-\mathrm{P} 2$ & $117.7(3)$ & $\begin{array}{l}\mathrm{H} 53 \mathrm{~A}-\mathrm{C} 53- \\
\mathrm{H} 53 \mathrm{~B}\end{array}$ & 107.8 \\
\hline $\mathrm{C} 22-\mathrm{C} 23-\mathrm{C} 24$ & $120.9(4)$ & $\mathrm{C} 43-01-\mathrm{Os} 1$ & $122.8(3)$ \\
\hline $\mathrm{C} 22-\mathrm{C} 23-\mathrm{H} 23$ & 119.5 & $\mathrm{C} 43-\mathrm{O} 2-\mathrm{Os} 2$ & $125.0(3)$ \\
\hline $\mathrm{C} 24-\mathrm{C} 23-\mathrm{H} 23$ & 119.5 & $\mathrm{C} 46-\mathrm{O} 3-\mathrm{Os} 1$ & $126.2(3)$ \\
\hline $\mathrm{C} 23-\mathrm{C} 24-\mathrm{C} 25$ & $120.7(4)$ & $\mathrm{C} 46-\mathrm{O} 4-\mathrm{Os} 2$ & $121.5(3)$ \\
\hline $\mathrm{C} 23-\mathrm{C} 24-\mathrm{H} 24$ & 119.7 & C50-Os1-C49 & $89.3(2)$ \\
\hline $\mathrm{C} 25-\mathrm{C} 24-\mathrm{H} 24$ & 119.7 & $\mathrm{C} 50-\mathrm{Os} 1-\mathrm{O} 3$ & $176.98(17)$ \\
\hline $\mathrm{C} 24-\mathrm{C} 25-\mathrm{C} 26$ & $118.2(4)$ & $\mathrm{C} 49-\mathrm{Os} 1-\mathrm{O} 3$ & $93.43(17)$ \\
\hline $\mathrm{C} 24-\mathrm{C} 25-\mathrm{C} 28$ & $120.9(4)$ & $\mathrm{C} 50-\mathrm{Os} 1-\mathrm{O} 1$ & $95.18(17)$ \\
\hline $\mathrm{C} 26-\mathrm{C} 25-\mathrm{C} 28$ & $120.8(4)$ & $\mathrm{C} 49-\mathrm{Os} 1-\mathrm{O} 1$ & $175.29(17)$ \\
\hline $\mathrm{C} 27-\mathrm{C} 26-\mathrm{C} 25$ & $120.8(4)$ & $\mathrm{O} 3-\mathrm{Os} 1-\mathrm{O} 1$ & $82.10(13)$ \\
\hline $\mathrm{C} 27-\mathrm{C} 26-\mathrm{H} 26$ & 119.6 & $\mathrm{C} 50-\mathrm{Os} 1-\mathrm{P} 1$ & $94.55(13)$ \\
\hline $\mathrm{C} 25-\mathrm{C} 26-\mathrm{H} 26$ & 119.6 & $\mathrm{C} 49-\mathrm{O} 1-\mathrm{P} 1$ & $96.01(14)$ \\
\hline $\mathrm{C} 26-\mathrm{C} 27-\mathrm{C} 22$ & $120.7(4)$ & $\mathrm{O} 3-\mathrm{Os} 1-\mathrm{P} 1$ & $86.56(9)$ \\
\hline $\mathrm{C} 26-\mathrm{C} 27-\mathrm{H} 27$ & 119.6 & $\mathrm{O} 1-\mathrm{Os} 1-\mathrm{P} 1$ & $85.24(9)$ \\
\hline $\mathrm{C} 22-\mathrm{C} 27-\mathrm{H} 27$ & 119.6 & C50-Os1-Os2 & $97.03(13)$ \\
\hline $\mathrm{C} 25-\mathrm{C} 28-\mathrm{H} 28 \mathrm{~A}$ & 109.5 & C49-Os1-Os2 & $94.85(14)$ \\
\hline
\end{tabular}




\begin{tabular}{|c|c|c|c|}
\hline $\mathrm{C} 25-\mathrm{C} 28-\mathrm{H} 28 \mathrm{~B}$ & 109.5 & O3-Os1-Os2 & $81.38(8)$ \\
\hline $\mathrm{H} 28 \mathrm{~A}-\mathrm{C} 28-\mathrm{H} 28 \mathrm{~B}$ & 109.5 & O1-Os1-Os2 & $83.06(8)$ \\
\hline $\mathrm{C} 25-\mathrm{C} 28-\mathrm{H} 28 \mathrm{C}$ & 109.5 & $\mathrm{P} 1-\mathrm{Os} 1-\mathrm{Os} 2$ & $164.21(3)$ \\
\hline $\mathrm{H} 28 \mathrm{~A}-\mathrm{C} 28-\mathrm{H} 28 \mathrm{C}$ & 109.5 & C52-Os2-C51 & $90.2(2)$ \\
\hline $\mathrm{H} 28 \mathrm{~B}-\mathrm{C} 28-\mathrm{H} 28 \mathrm{C}$ & 109.5 & $\mathrm{C} 52-\mathrm{Os} 2-\mathrm{O} 2$ & $95.23(16)$ \\
\hline C34-C29-C30 & $118.2(4)$ & $\mathrm{C} 51-\mathrm{Os} 2-\mathrm{O} 2$ & $174.03(17)$ \\
\hline C34-C29-P2 & $122.6(3)$ & C52-Os2-O4 & $174.58(15)$ \\
\hline C30-C29-P2 & $119.1(3)$ & $\mathrm{C} 51-\mathrm{Os} 2-\mathrm{O} 4$ & $93.70(17)$ \\
\hline C31-C30-C29 & $121.2(4)$ & $\mathrm{O} 2-\mathrm{Os} 2-\mathrm{O} 4$ & $80.69(13)$ \\
\hline $\mathrm{C} 31-\mathrm{C} 30-\mathrm{H} 30$ & 119.4 & $\mathrm{C} 52-\mathrm{Os} 2-\mathrm{P} 2$ & $93.35(13)$ \\
\hline $\mathrm{C} 29-\mathrm{C} 30-\mathrm{H} 30$ & 119.4 & $\mathrm{C} 51-\mathrm{Os} 2-\mathrm{P} 2$ & $97.51(14)$ \\
\hline C30-C31-C32 & $120.8(4)$ & $\mathrm{O} 2-\mathrm{Os} 2-\mathrm{P} 2$ & $84.59(9)$ \\
\hline C30-C31-H31 & 119.6 & $\mathrm{O} 4-\mathrm{Os} 2-\mathrm{P} 2$ & $89.83(9)$ \\
\hline $\mathrm{C} 32-\mathrm{C} 31-\mathrm{H} 31$ & 119.6 & C52-Os2-Os1 & $91.74(13)$ \\
\hline $\mathrm{C} 33-\mathrm{C} 32-\mathrm{C} 31$ & $117.9(4)$ & C51-Os2-Os1 & $95.39(13)$ \\
\hline C33-C32-C35 & $120.9(5)$ & $\mathrm{O} 2-\mathrm{Os} 2-\mathrm{Os} 1$ & $82.07(9)$ \\
\hline C31-C32-C35 & $121.2(4)$ & O4-Os2-Os1 & $84.19(8)$ \\
\hline C32-C33-C34 & $121.4(5)$ & $\mathrm{P} 2-\mathrm{Os} 2-\mathrm{Os} 1$ & $166.10(3)$ \\
\hline C32-C33-H33 & 119.3 & $\mathrm{C} 8-\mathrm{P} 1-\mathrm{C} 15$ & $103.4(2)$ \\
\hline C34-C33-H33 & 119.3 & $\mathrm{C} 8-\mathrm{P} 1-\mathrm{C} 1$ & $101.52(19)$ \\
\hline $\mathrm{C} 29-\mathrm{C} 34-\mathrm{C} 33$ & $120.5(4)$ & $\mathrm{C} 15-\mathrm{P} 1-\mathrm{C} 1$ & $103.44(19)$ \\
\hline C29-C34-H34 & 119.8 & $\mathrm{C} 8-\mathrm{P} 1-\mathrm{Os} 1$ & $116.39(14)$ \\
\hline C33-C34-H34 & 119.8 & $\mathrm{C} 15-\mathrm{P} 1-\mathrm{Os} 1$ & $112.59(14)$ \\
\hline C32-C35-H35A & 109.5 & $\mathrm{C} 1-\mathrm{P} 1-\mathrm{O} 1$ & $117.63(14)$ \\
\hline C32-C35-H35B & 109.5 & C36-P2-C22 & $104.9(2)$ \\
\hline
\end{tabular}




\begin{tabular}{|c|c|c|c|}
\hline $\mathrm{H} 35 \mathrm{~A}-\mathrm{C} 35-\mathrm{H} 35 \mathrm{~B}$ & 109.5 & C36-P2-C29 & $102.2(2)$ \\
\hline $\mathrm{C} 32-\mathrm{C} 35-\mathrm{H} 35 \mathrm{C}$ & 109.5 & $\mathrm{C} 22-\mathrm{P} 2-\mathrm{C} 29$ & $101.4(2)$ \\
\hline $\mathrm{H} 35 \mathrm{~A}-\mathrm{C} 35-\mathrm{H} 35 \mathrm{C}$ & 109.5 & $\mathrm{C} 36-\mathrm{P} 2-\mathrm{Os} 2$ & $111.30(15)$ \\
\hline H35B-C35-H35C & 109.5 & C22-P2-Os2 & $117.41(15)$ \\
\hline $\mathrm{C} 41-\mathrm{C} 36-\mathrm{C} 37$ & $118.4(4)$ & $\mathrm{C} 29-\mathrm{P} 2-\mathrm{Os} 2$ & $117.74(15)$ \\
\hline $\mathrm{C} 6-\mathrm{C} 1-\mathrm{C} 2-\mathrm{C} 3$ & $1.6(7)$ & $\begin{array}{l}\text { C38-C39-C40- } \\
\text { C41 }\end{array}$ & $-0.5(7)$ \\
\hline $\mathrm{P} 1-\mathrm{C} 1-\mathrm{C} 2-\mathrm{C} 3$ & $-177.7(4)$ & $\begin{array}{l}\text { C42-C39-C40- } \\
\text { C41 }\end{array}$ & $-179.0(5)$ \\
\hline $\mathrm{C} 1-\mathrm{C} 2-\mathrm{C} 3-\mathrm{C} 4$ & $0.2(7)$ & $\begin{array}{l}\text { C39-C40-C41- } \\
\text { C36 }\end{array}$ & $-1.6(7)$ \\
\hline $\mathrm{C} 2-\mathrm{C} 3-\mathrm{C} 4-\mathrm{C} 5$ & $-1.9(7)$ & $\begin{array}{l}\mathrm{C} 37-\mathrm{C} 36-\mathrm{C} 41- \\
\mathrm{C} 40\end{array}$ & $1.7(7)$ \\
\hline $\mathrm{C} 2-\mathrm{C} 3-\mathrm{C} 4-\mathrm{C} 7$ & $177.0(4)$ & $\begin{array}{l}\mathrm{P} 2-\mathrm{C} 36-\mathrm{C} 41- \\
\mathrm{C} 40\end{array}$ & $174.1(4)$ \\
\hline $\mathrm{C} 3-\mathrm{C} 4-\mathrm{C} 5-\mathrm{C} 6$ & $1.9(7)$ & $\begin{array}{l}\text { O1-C43-C44- } \\
\text { C45 }\end{array}$ & $-4.3(8)$ \\
\hline $\mathrm{C} 7-\mathrm{C} 4-\mathrm{C} 5-\mathrm{C} 6$ & $-177.0(4)$ & $\begin{array}{l}\mathrm{O} 2-\mathrm{C} 43-\mathrm{C} 44- \\
\mathrm{C} 45\end{array}$ & $177.7(6)$ \\
\hline $\mathrm{C} 2-\mathrm{C} 1-\mathrm{C} 6-\mathrm{C} 5$ & $-1.6(7)$ & $\begin{array}{l}\text { O1-C43-C44- } \\
\text { C45A }\end{array}$ & $50.5(14)$ \\
\hline $\mathrm{P} 1-\mathrm{C} 1-\mathrm{C} 6-\mathrm{C} 5$ & $177.7(4)$ & $\begin{array}{l}\mathrm{O} 2-\mathrm{C} 43-\mathrm{C} 44- \\
\mathrm{C} 45 \mathrm{~A}\end{array}$ & $-127.6(14)$ \\
\hline $\mathrm{C} 4-\mathrm{C} 5-\mathrm{C} 6-\mathrm{C} 1$ & $-0.2(7)$ & $\begin{array}{l}\text { O4-C46-C47- } \\
\text { C48 }\end{array}$ & $107.9(5)$ \\
\hline $\mathrm{C} 13-\mathrm{C} 8-\mathrm{C} 9-\mathrm{C} 10$ & $-2.1(6)$ & $\begin{array}{l}\text { O3-C46-C47- } \\
\text { C48 }\end{array}$ & $-71.4(5)$ \\
\hline
\end{tabular}




\begin{tabular}{|c|c|c|c|}
\hline $\mathrm{P} 1-\mathrm{C} 8-\mathrm{C} 9-\mathrm{C} 10$ & $173.0(3)$ & $\begin{array}{l}\mathrm{O} 2-\mathrm{C} 43-\mathrm{O} 1- \\
\text { Os1 }\end{array}$ & $10.8(7)$ \\
\hline $\mathrm{C} 8-\mathrm{C} 9-\mathrm{C} 10-\mathrm{C} 11$ & $2.5(7)$ & $\begin{array}{l}\text { C44-C43-O1- } \\
\text { Os1 }\end{array}$ & $-167.0(3)$ \\
\hline $\begin{array}{l}\mathrm{C} 9-\mathrm{C} 10-\mathrm{C} 11- \\
\mathrm{C} 12\end{array}$ & $-0.6(7)$ & $\begin{array}{l}\mathrm{O} 1-\mathrm{C} 43-\mathrm{O} 2- \\
\text { Os2 }\end{array}$ & $0.5(7)$ \\
\hline $\begin{array}{l}\mathrm{C} 9-\mathrm{C} 10-\mathrm{C} 11- \\
\mathrm{C} 14\end{array}$ & $-177.8(4)$ & $\begin{array}{l}\text { C44-C43-O2- } \\
\text { Os2 }\end{array}$ & $178.4(3)$ \\
\hline $\begin{array}{l}\mathrm{C} 10-\mathrm{C} 11-\mathrm{C} 12- \\
\mathrm{C} 13\end{array}$ & $-1.6(7)$ & $\begin{array}{l}\mathrm{O} 4-\mathrm{C} 46-\mathrm{O} 3- \\
\text { Os1 }\end{array}$ & $-5.9(6)$ \\
\hline $\begin{array}{l}\text { C14-C11-C12- } \\
\text { C13 }\end{array}$ & $175.6(4)$ & $\begin{array}{l}\text { C47-C46-O3- } \\
\text { Os1 }\end{array}$ & $173.4(3)$ \\
\hline $\begin{array}{l}\mathrm{C} 11-\mathrm{C} 12-\mathrm{C} 13- \\
\mathrm{C} 8\end{array}$ & $2.0(7)$ & $\begin{array}{l}\text { O3-C46-O4- } \\
\text { Os2 }\end{array}$ & $14.4(6)$ \\
\hline $\mathrm{C} 9-\mathrm{C} 8-\mathrm{C} 13-\mathrm{C} 12$ & $-0.1(6)$ & $\begin{array}{l}\text { C47-C46-O4- } \\
\text { Os2 }\end{array}$ & $-164.8(3)$ \\
\hline $\mathrm{P} 1-\mathrm{C} 8-\mathrm{C} 13-\mathrm{C} 12$ & $-175.3(3)$ & C9-C8-P1-C15 & $27.8(4)$ \\
\hline $\begin{array}{l}\mathrm{C} 20-\mathrm{C} 15-\mathrm{C} 16- \\
\mathrm{C} 17\end{array}$ & $-2.3(7)$ & $\begin{array}{l}\mathrm{C} 13-\mathrm{C} 8-\mathrm{P} 1- \\
\mathrm{C} 15\end{array}$ & $-157.2(4)$ \\
\hline $\begin{array}{l}\mathrm{P} 1-\mathrm{C} 15-\mathrm{C} 16- \\
\mathrm{C} 17\end{array}$ & $-175.6(4)$ & $\mathrm{C} 9-\mathrm{C} 8-\mathrm{P} 1-\mathrm{C} 1$ & $-79.2(4)$ \\
\hline $\begin{array}{l}\text { C15-C16-C17- } \\
\text { C18 }\end{array}$ & $0.5(7)$ & $\mathrm{C} 13-\mathrm{C} 8-\mathrm{P} 1-\mathrm{C} 1$ & $95.8(4)$ \\
\hline $\begin{array}{l}\text { C16-C17-C18- } \\
\text { C19 }\end{array}$ & $0.5(7)$ & $\mathrm{C} 9-\mathrm{C} 8-\mathrm{P} 1-\mathrm{Os} 1$ & $151.8(3)$ \\
\hline $\begin{array}{l}\mathrm{C} 16-\mathrm{C} 17-\mathrm{C} 18- \\
\mathrm{C} 21\end{array}$ & $-179.1(5)$ & $\begin{array}{l}\mathrm{C} 13-\mathrm{C} 8-\mathrm{P} 1- \\
\text { Os1 }\end{array}$ & $-33.3(4)$ \\
\hline $\begin{array}{l}\text { C17-C18-C19- } \\
\text { C20 }\end{array}$ & $0.5(7)$ & $\begin{array}{l}\mathrm{C} 16-\mathrm{C} 15-\mathrm{P} 1- \\
\mathrm{C} 8\end{array}$ & $-132.7(4)$ \\
\hline
\end{tabular}




\begin{tabular}{|c|c|c|c|}
\hline $\begin{array}{l}\text { C21-C18-C19- } \\
\text { C20 }\end{array}$ & $180.0(4)$ & $\begin{array}{l}\mathrm{C} 20-\mathrm{C} 15-\mathrm{P} 1- \\
\mathrm{C} 8\end{array}$ & $54.1(4)$ \\
\hline $\begin{array}{l}\text { C18-C19-C20- } \\
\text { C15 }\end{array}$ & $-2.4(7)$ & $\begin{array}{l}\mathrm{C} 16-\mathrm{C} 15-\mathrm{P} 1- \\
\mathrm{C} 1\end{array}$ & $-27.2(4)$ \\
\hline $\begin{array}{l}\mathrm{C} 16-\mathrm{C} 15-\mathrm{C} 20- \\
\mathrm{C} 19\end{array}$ & $3.2(7)$ & $\begin{array}{l}\mathrm{C} 20-\mathrm{C} 15-\mathrm{P} 1- \\
\mathrm{C} 1\end{array}$ & $159.7(3)$ \\
\hline $\begin{array}{l}\mathrm{P} 1-\mathrm{C} 15-\mathrm{C} 20- \\
\mathrm{C} 19\end{array}$ & $176.8(3)$ & $\begin{array}{l}\text { C16-C15-P1- } \\
\text { Os1 }\end{array}$ & $100.9(4)$ \\
\hline $\begin{array}{l}\text { C27-C22-C23- } \\
\text { C24 }\end{array}$ & $1.3(7)$ & $\begin{array}{l}\text { C20-C15-P1- } \\
\text { Os1 }\end{array}$ & $-72.3(4)$ \\
\hline $\begin{array}{l}\text { P2-C22-C23- } \\
\text { C24 }\end{array}$ & $-172.8(4)$ & $\mathrm{C} 6-\mathrm{C} 1-\mathrm{P} 1-\mathrm{C} 8$ & $46.7(4)$ \\
\hline $\begin{array}{l}\text { C22-C23-C24- } \\
\text { C25 }\end{array}$ & $0.8(7)$ & $\mathrm{C} 2-\mathrm{C} 1-\mathrm{P} 1-\mathrm{C} 8$ & $-134.1(4)$ \\
\hline $\begin{array}{l}\text { C23-C24-C25- } \\
\text { C26 }\end{array}$ & $-2.7(7)$ & $\mathrm{C} 6-\mathrm{C} 1-\mathrm{P} 1-\mathrm{C} 15$ & $-60.3(4)$ \\
\hline $\begin{array}{l}\text { C23-C24-C25- } \\
\text { C28 }\end{array}$ & $176.8(4)$ & $\mathrm{C} 2-\mathrm{C} 1-\mathrm{P} 1-\mathrm{C} 15$ & $118.9(4)$ \\
\hline $\begin{array}{l}\mathrm{C} 24-\mathrm{C} 25-\mathrm{C} 26- \\
\mathrm{C} 27\end{array}$ & $2.5(7)$ & $\mathrm{C} 6-\mathrm{C} 1-\mathrm{P} 1-\mathrm{Os} 1$ & $174.9(3)$ \\
\hline $\begin{array}{l}\mathrm{C} 28-\mathrm{C} 25-\mathrm{C} 26- \\
\mathrm{C} 27\end{array}$ & $-176.9(4)$ & $\mathrm{C} 2-\mathrm{C} 1-\mathrm{P} 1-\mathrm{Os} 1$ & $-5.9(4)$ \\
\hline $\begin{array}{l}\text { C25-C26-C27- } \\
\text { C22 }\end{array}$ & $-0.5(7)$ & $\begin{array}{l}\text { C41-C36-P2- } \\
\text { C22 }\end{array}$ & $130.3(4)$ \\
\hline $\begin{array}{l}\text { C23-C22-C27- } \\
\text { C26 }\end{array}$ & $-1.5(7)$ & $\begin{array}{l}\text { C37-C36-P2- } \\
\text { C22 }\end{array}$ & $-57.4(4)$ \\
\hline $\begin{array}{l}\text { P2-C22-C27- } \\
\text { C26 }\end{array}$ & $173.0(3)$ & $\begin{array}{l}\text { C41-C36-P2- } \\
\text { C29 }\end{array}$ & $24.9(4)$ \\
\hline C34-C29-C30- & $1.5(7)$ & C37-C36-P2- & $-162.8(4)$ \\
\hline
\end{tabular}




\begin{tabular}{|c|c|c|c|}
\hline C31 & & C29 & \\
\hline $\begin{array}{l}\mathrm{P} 2-\mathrm{C} 29-\mathrm{C} 30- \\
\mathrm{C} 31\end{array}$ & $-177.7(4)$ & $\begin{array}{l}\text { C41-C36-P2- } \\
\text { Os2 }\end{array}$ & $-101.7(4)$ \\
\hline $\begin{array}{l}\mathrm{C} 29-\mathrm{C} 30-\mathrm{C} 31- \\
\mathrm{C} 32\end{array}$ & $-2.3(7)$ & $\begin{array}{l}\mathrm{C} 37-\mathrm{C} 36-\mathrm{P} 2- \\
\text { Os2 }\end{array}$ & $70.6(4)$ \\
\hline $\begin{array}{l}\text { C30-C31-C32- } \\
\text { C33 }\end{array}$ & $1.6(7)$ & $\begin{array}{l}\mathrm{C} 23-\mathrm{C} 22-\mathrm{P} 2- \\
\mathrm{C} 36\end{array}$ & $-16.3(4)$ \\
\hline $\begin{array}{l}\text { C30-C31-C32- } \\
\text { C35 }\end{array}$ & $-178.9(5)$ & $\begin{array}{l}\mathrm{C} 27-\mathrm{C} 22-\mathrm{P} 2- \\
\mathrm{C} 36\end{array}$ & $169.6(3)$ \\
\hline $\begin{array}{l}\text { C31-C32-C33- } \\
\text { C34 }\end{array}$ & $-0.4(7)$ & $\begin{array}{l}\mathrm{C} 23-\mathrm{C} 22-\mathrm{P} 2- \\
\mathrm{C} 29\end{array}$ & $89.8(4)$ \\
\hline $\begin{array}{l}\text { C35-C32-C33- } \\
\text { C34 }\end{array}$ & $-179.9(5)$ & $\begin{array}{l}\mathrm{C} 27-\mathrm{C} 22-\mathrm{P} 2- \\
\mathrm{C} 29\end{array}$ & $-84.3(4)$ \\
\hline $\begin{array}{l}\text { C30-C29-C34- } \\
\text { C33 }\end{array}$ & $-0.3(7)$ & $\begin{array}{l}\text { C23-C22-P2- } \\
\text { Os2 }\end{array}$ & $-140.4(3)$ \\
\hline $\begin{array}{l}\mathrm{P} 2-\mathrm{C} 29-\mathrm{C} 34- \\
\mathrm{C} 33\end{array}$ & $178.9(4)$ & $\begin{array}{l}\mathrm{C} 27-\mathrm{C} 22-\mathrm{P} 2- \\
\text { Os2 }\end{array}$ & $45.4(4)$ \\
\hline $\begin{array}{l}\text { C32-C33-C34- } \\
\text { C29 }\end{array}$ & $-0.3(7)$ & $\begin{array}{l}\mathrm{C} 34-\mathrm{C} 29-\mathrm{P} 2- \\
\mathrm{C} 36\end{array}$ & $-120.5(4)$ \\
\hline $\begin{array}{l}\text { C41-C36-C37- } \\
\text { C38 }\end{array}$ & $0.3(7)$ & $\begin{array}{l}\mathrm{C} 30-\mathrm{C} 29-\mathrm{P} 2- \\
\mathrm{C} 36\end{array}$ & $58.7(4)$ \\
\hline $\begin{array}{l}\mathrm{P} 2-\mathrm{C} 36-\mathrm{C} 37- \\
\mathrm{C} 38\end{array}$ & $-172.3(4)$ & $\begin{array}{l}\mathrm{C} 34-\mathrm{C} 29-\mathrm{P} 2- \\
\mathrm{C} 22\end{array}$ & $131.3(4)$ \\
\hline $\begin{array}{l}\text { C36-C37-C38- } \\
\text { C39 }\end{array}$ & $-2.4(8)$ & $\begin{array}{l}\mathrm{C} 30-\mathrm{C} 29-\mathrm{P} 2- \\
\mathrm{C} 22\end{array}$ & $-49.5(4)$ \\
\hline $\begin{array}{l}\text { C37-C38-C39- } \\
\text { C40 }\end{array}$ & $2.5(8)$ & $\begin{array}{l}\text { C34-C29-P2- } \\
\text { Os2 }\end{array}$ & $1.8(4)$ \\
\hline $\begin{array}{l}\text { C37-C38-C39- } \\
\text { C42 }\end{array}$ & $-179.0(5)$ & $\begin{array}{l}\text { C30-C29-P2- } \\
\text { Os2 }\end{array}$ & $-179.0(3)$ \\
\hline
\end{tabular}


(8)

Crystal data

\begin{tabular}{|c|c|}
\hline $\mathrm{C}_{48} \mathrm{H}_{44} \mathrm{O}_{8} \mathrm{Os}_{2} \mathrm{P}_{2}$ & $Z=2$ \\
\hline$M_{r}=1191.17$ & $F(000)=1156$ \\
\hline Triclinic, $P \overline{1}$ & $D_{\mathrm{x}}=1.747 \mathrm{Mg} \mathrm{m}^{-3}$ \\
\hline$a=10.7707(4) \AA$ & Mo $K \alpha$ radiation, $\lambda=0.71073 \AA$ \\
\hline$b=10.8014(4) \AA$ & Cell parameters from 9955 reflections \\
\hline$c=20.3790(7) \AA$ & $\theta=2.5-27.1^{\circ}$ \\
\hline$\alpha=104.393(1)^{\circ}$ & $\mu=5.73 \mathrm{~mm}^{-1}$ \\
\hline$\beta=91.009(1)^{\circ}$ & $T=200 \mathrm{~K}$ \\
\hline$\gamma=98.993(1)^{\circ}$ & Block, colorless \\
\hline$V=2264.27(14) \AA^{3}$ & $0.15 \times 0.11 \times 0.04 \mathrm{~mm}$ \\
\hline
\end{tabular}

Data collection

\begin{tabular}{|l|l|}
\hline Bruker Apexll CCD diffractometer & 8554 reflections with $I>2 \sigma(I)$ \\
\hline$\phi$ and $\omega$ scans & $R_{\text {int }}=0.058$ \\
\hline $\begin{array}{l}\text { Absorption correction: multi-scan } \\
\text { SADABS (Bruker, 2008) }\end{array}$ & $\theta_{\max }=27.1^{\circ}, \theta_{\min }=1.0^{\circ}$ \\
\hline$T_{\min }=0.538, T_{\max }=0.746$ & $h=-13 \rightarrow 13$ \\
\hline 30910 measured reflections & $k=-13 \rightarrow 13$ \\
\hline 9960 independent reflections & $I=-26 \rightarrow 26$ \\
\hline
\end{tabular}

Refinement

Refinement on $F^{2}$

Hydrogen site location: inferred from neighbouring sites 


\begin{tabular}{|c|c|}
\hline Least-squares matrix: full & $\mathrm{H}$-atom parameters constrained \\
\hline$R\left[F^{2}>2 \sigma\left(F^{2}\right)\right]=0.030$ & $\begin{array}{l}w=1 /\left[\sigma^{2}\left(F_{0}^{2}\right)+(0.0249 P)^{2}+1.5967 P\right] \\
\text { where } P=\left(F_{0}^{2}+2 F_{\mathrm{c}}^{2}\right) / 3\end{array}$ \\
\hline$w R\left(F^{2}\right)=0.082$ & $(\Delta / \sigma)_{\max }=0.002$ \\
\hline$S=1.06$ & 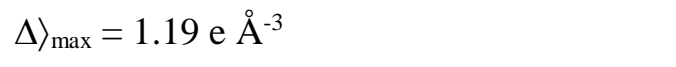 \\
\hline 9960 reflections & $\Delta\rangle_{\min }=-1.09 \mathrm{e} \AA^{-3}$ \\
\hline 548 parameters & $\begin{array}{l}\text { Extinction correction: SHELXL2018/3 } \\
\text { (Sheldrick 2018), } \\
\mathrm{Fc}^{*}=\mathrm{kFc}\left[1+0.001 \mathrm{xFc}^{2} \lambda^{3} / \sin (2 \theta)\right]^{-1 / 4}\end{array}$ \\
\hline 0 restraints & Extinction coefficient: $0.00032(9)$ \\
\hline
\end{tabular}

\section{Special details}

Geometry. All esds (except the esd in the dihedral angle between two I.s. planes) are estimated using the full covariance matrix. The cell esds are taken into account individually in the estimation of esds in distances, angles and torsion angles; correlations between esds in cell parameters are only used when they are defined by crystal symmetry. An approximate (isotropic) treatment of cell esds is used for estimating esds involving I.s. planes.

Fractional atomic coordinates and isotropic or equivalent isotropic displacement parameters $\left(\AA^{2}\right)$ for (8)

\begin{tabular}{|l|l|l|l|l|}
\hline & $x$ & $y$ & $z$ & $U_{\text {iso }} / U_{\text {eq }}$ \\
\hline Os1 & $0.69417(2)$ & $0.59590(2)$ & $0.29383(2)$ & $0.02493(6)$ \\
\hline P1 & $0.81773(10)$ & $0.76444(10)$ & $0.38172(5)$ & $0.0238(2)$ \\
\hline O1 & $0.6478(4)$ & $0.7406(3)$ & $0.24699(18)$ & $0.0461(9)$ \\
\hline C1 & $0.5899(6)$ & $0.7107(5)$ & $0.1902(3)$ & $0.0508(14)$ \\
\hline H1 & 0.587681 & 0.779927 & 0.169394 & $0.061^{*}$ \\
\hline Os2 & $0.50480(2)$ & $0.44535(2)$ & $0.20412(2)$ & $0.02496(6)$ \\
\hline
\end{tabular}




\begin{tabular}{|c|c|c|c|c|}
\hline $\mathrm{O} 2$ & $0.5349(3)$ & $0.6028(3)$ & $0.15726(16)$ & $0.0386(8)$ \\
\hline P2 & $0.32402(10)$ & $0.36297(11)$ & $0.12322(5)$ & $0.0254(2)$ \\
\hline C2 & $0.4312(5)$ & $0.6229(5)$ & $0.3295(2)$ & $0.0422(12)$ \\
\hline $\mathrm{H} 2$ & 0.371286 & 0.660669 & 0.358300 & $0.051^{*}$ \\
\hline C3 & $0.8164(5)$ & $0.5635(5)$ & $0.2324(3)$ & $0.0435(12)$ \\
\hline $\mathrm{O} 3$ & $0.5407(3)$ & $0.6400(3)$ & $0.35564(16)$ & $0.0374(8)$ \\
\hline C4 & $0.7314(5)$ & $0.4638(5)$ & $0.3293(3)$ & $0.0392(11)$ \\
\hline O4 & $0.3907(3)$ & $0.5630(3)$ & $0.27093(16)$ & $0.0389(8)$ \\
\hline O5 & $0.8900(4)$ & $0.5444(5)$ & $0.1927(2)$ & $0.0729(15)$ \\
\hline C5 & $0.6153(5)$ & $0.3528(5)$ & $0.1529(2)$ & $0.0381(11)$ \\
\hline $\mathrm{O} 6$ & $0.7552(4)$ & 0.3798 (4) & $0.3495(2)$ & $0.0662(13)$ \\
\hline C6 & $0.4812(5)$ & $0.3237(5)$ & $0.2534(3)$ & $0.0408(12)$ \\
\hline $\mathrm{O} 7$ & $0.6866(4)$ & $0.2952(4)$ & $0.1227(2)$ & $0.0571(11)$ \\
\hline $\mathrm{C} 7$ & $0.7472(4)$ & $0.9097(4)$ & $0.4120(2)$ & $0.0261(9)$ \\
\hline C8 & $0.7160(4)$ & $0.9755(4)$ & $0.3650(2)$ & $0.0302(9)$ \\
\hline $\mathrm{H} 8$ & 0.730434 & 0.943097 & 0.318522 & $0.036^{*}$ \\
\hline O8 & $0.4676(5)$ & $0.2497(4)$ & $0.2865(2)$ & $0.0745(15)$ \\
\hline $\mathrm{C9}$ & $0.6641(4)$ & $1.0875(4)$ & $0.3851(2)$ & $0.0317(10)$ \\
\hline H9 & 0.645251 & 1.131966 & 0.352375 & $0.038^{*}$ \\
\hline C10 & $0.6394(5)$ & $1.1354(5)$ & $0.4520(3)$ & $0.0383(11)$ \\
\hline C11 & $0.6713(6)$ & $1.0704(5)$ & $0.4985(3)$ & 0.0531 \\
\hline $\mathrm{H} 11$ & 0.656524 & 1.103077 & 0.544958 & $0.064^{*}$ \\
\hline C12 & $0.7240(6)$ & $0.9594(5)$ & $0.4795(2)$ & 0.0449 (13) \\
\hline $\mathrm{H} 12$ & 0.744483 & 0.916591 & 0.512723 & $0.054^{*}$ \\
\hline C13 & $0.9740(4)$ & $0.8264(4)$ & $0.3597(2)$ & $0.0266(9)$ \\
\hline
\end{tabular}




\begin{tabular}{|c|c|c|c|c|}
\hline C14 & $1.0236(4)$ & $0.9585(4)$ & $0.3734(2)$ & $0.0325(10)$ \\
\hline $\mathrm{H} 14$ & 0.975167 & 1.021349 & 0.395397 & $0.039^{*}$ \\
\hline C15 & $1.1443(5)$ & $0.9977(5)$ & $0.3546(3)$ & $0.0400(11)$ \\
\hline $\mathrm{H} 15$ & 1.176412 & 1.087678 & 0.363518 & $0.048^{*}$ \\
\hline C16 & $1.2181(5)$ & $0.9099(6)$ & $0.3235(3)$ & $0.0424(12)$ \\
\hline C17 & $1.1687(5)$ & $0.7792(5)$ & $0.3113(2)$ & $0.0393(11)$ \\
\hline $\mathrm{H} 17$ & 1.219089 & 0.716909 & 0.290980 & $0.047^{*}$ \\
\hline C18 & $1.0491(4)$ & $0.7374(5)$ & $0.3278(2)$ & $0.0339(10)$ \\
\hline $\mathrm{H} 18$ & 1.017219 & 0.647215 & 0.317486 & $0.041^{*}$ \\
\hline C19 & $0.8480(4)$ & $0.7134(4)$ & $0.4589(2)$ & $0.0266(9)$ \\
\hline $\mathrm{C} 20$ & $0.7464(5)$ & $0.6515(5)$ & $0.4866(2)$ & $0.0361(10)$ \\
\hline $\mathrm{H} 2 \mathrm{O}$ & 0.665145 & 0.634224 & 0.464179 & $0.043^{*}$ \\
\hline $\mathrm{C} 21$ & $0.7618(5)$ & $0.6148(5)$ & $0.5460(3)$ & $0.0416(12)$ \\
\hline $\mathrm{H} 21$ & 0.690740 & 0.575966 & 0.564927 & $0.050^{*}$ \\
\hline $\mathrm{C} 22$ & $0.8811(5)$ & $0.6343(5)$ & $0.5784(3)$ & $0.0430(12)$ \\
\hline $\mathrm{C} 23$ & $0.9822(5)$ & $0.6931(5)$ & $0.5503(3)$ & $0.0438(12)$ \\
\hline $\mathrm{H} 23$ & 1.064031 & 0.706989 & 0.571739 & $0.053^{*}$ \\
\hline C24 & $0.9663(4)$ & $0.7326(4)$ & $0.4905(2)$ & $0.0334(10)$ \\
\hline $\mathrm{H} 24$ & 1.037121 & 0.772633 & 0.471843 & $0.040^{*}$ \\
\hline C25 & $0.2555(4)$ & $0.4836(4)$ & 0.0925 (2) & $0.0294(9)$ \\
\hline C26 & $0.2262(5)$ & $0.4730(5)$ & $0.0242(2)$ & $0.0369(11)$ \\
\hline H26 & 0.244275 & 0.400683 & -0.009421 & $0.044^{*}$ \\
\hline $\mathrm{C} 27$ & $0.1711(5)$ & $0.5664(5)$ & $0.0050(3)$ & $0.0439(12)$ \\
\hline $\mathrm{H} 27$ & 0.152324 & 0.557505 & -0.041832 & $0.053^{*}$ \\
\hline $\mathrm{C} 28$ & $0.1428(5)$ & $0.6716(5)$ & $0.0515(3)$ & $0.0409(12)$ \\
\hline
\end{tabular}




\begin{tabular}{|c|c|c|c|c|}
\hline C29 & $0.1717(5)$ & $0.6832(5)$ & $0.1202(3)$ & $0.0464(13)$ \\
\hline H29 & 0.152913 & 0.755572 & 0.153414 & $0.056^{*}$ \\
\hline C30 & $0.2271(5)$ & $0.5909(5)$ & $0.1400(3)$ & $0.0432(12)$ \\
\hline $\mathrm{H} 30$ & 0.246246 & 0.600576 & 0.186823 & $0.052^{*}$ \\
\hline C31 & $0.1902(4)$ & $0.2697(4)$ & $0.1517(2)$ & $0.0290(9)$ \\
\hline C32 & $0.2086(4)$ & $0.1642(5)$ & $0.1759(2)$ & $0.0347(10)$ \\
\hline H32 & 0.291382 & 0.145594 & 0.179533 & $0.042^{*}$ \\
\hline C33 & $0.1090(5)$ & $0.0854(6)$ & $0.1951(3)$ & $0.0460(13)$ \\
\hline H33 & 0.123817 & 0.012699 & 0.210535 & $0.055^{*}$ \\
\hline C34 & $-0.0121(5)$ & $0.1127(8)$ & $0.1916(3)$ & $0.0634(19)$ \\
\hline C35 & $-0.0309(5)$ & $0.2184(8)$ & $0.1704(3)$ & $0.067(2)$ \\
\hline H35 & -0.113138 & 0.239727 & 0.169983 & $0.080^{*}$ \\
\hline C36 & $0.0684(5)$ & $0.2966(6)$ & $0.1491(3)$ & $0.0458(13)$ \\
\hline H36 & 0.052460 & 0.368229 & 0.132960 & $0.055^{*}$ \\
\hline C37 & $0.3638(4)$ & $0.2534(4)$ & $0.0457(2)$ & $0.0277(9)$ \\
\hline C38 & $0.2969(5)$ & $0.1299(5)$ & $0.0181(2)$ & $0.0383(11)$ \\
\hline H38 & 0.222740 & 0.100150 & 0.037977 & $0.046^{*}$ \\
\hline C39 & $0.3387(6)$ & $0.0496(5)$ & $-0.0390(3)$ & $0.0464(13)$ \\
\hline H39 & 0.292376 & -0.034764 & -0.057637 & $0.056^{*}$ \\
\hline C40 & $0.4462(5)$ & $0.0907(5)$ & $-0.0687(2)$ & $0.0386(11)$ \\
\hline C41 & $0.5085(5)$ & $0.2152(5)$ & $-0.0420(2)$ & $0.0388(11)$ \\
\hline $\mathrm{H} 41$ & 0.580414 & 0.246334 & -0.063274 & $0.047^{*}$ \\
\hline C42 & $0.4697(4)$ & $0.2957(5)$ & $0.0144(2)$ & $0.0355(10)$ \\
\hline $\mathrm{H} 42$ & 0.515520 & 0.380576 & 0.032129 & $0.043^{*}$ \\
\hline C43 & $0.5805(7)$ & $1.2550(6)$ & $0.4731(3)$ & $0.0644(18)$ \\
\hline
\end{tabular}




\begin{tabular}{|l|l|l|l|l|}
\hline H43A & 0.597271 & 1.292723 & 0.521960 & $0.097^{\star}$ \\
\hline H43B & 0.489365 & 1.232428 & 0.462586 & $0.097^{\star}$ \\
\hline H43C & 0.616319 & 1.318136 & 0.448422 & $0.097^{\star}$ \\
\hline C44 & $1.3501(6)$ & $0.9517(7)$ & $0.3040(4)$ & $0.0677(19)$ \\
\hline H44A & 1.408076 & 0.903532 & 0.320817 & $0.102^{\star}$ \\
\hline H44B & 1.376363 & 1.044780 & 0.324159 & $0.102^{\star}$ \\
\hline H44C & 1.351387 & 0.933956 & 0.254512 & $0.102^{\star}$ \\
\hline C45 & $0.8984(7)$ & $0.5927(7)$ & $0.6431(3)$ & $0.0692(19)$ \\
\hline H45A & 0.988432 & 0.598946 & 0.654205 & $0.104^{\star}$ \\
\hline H45B & 0.856481 & 0.502846 & 0.636818 & $0.104^{\star}$ \\
\hline H45C & 0.861447 & 0.649194 & 0.680233 & $0.104^{\star}$ \\
\hline C46 & $0.0825(7)$ & $0.7754(6)$ & $0.0301(3)$ & $0.0637(18)$ \\
\hline H46A & 0.115889 & 0.787900 & -0.012636 & $0.096^{\star}$ \\
\hline H46B & 0.101759 & 0.857079 & 0.065351 & $0.096^{\star}$ \\
\hline H46C & -0.008993 & 0.747778 & 0.023794 & $0.096^{\star}$ \\
\hline C47 & $-0.1216(6)$ & $0.0281(11)$ & $0.2136(5)$ & $0.118(4)$ \\
\hline H47A & -0.199251 & 0.030386 & 0.188434 & $0.177^{\star}$ \\
\hline H47B & -0.130619 & 0.060599 & 0.262317 & $0.177^{\star}$ \\
\hline H47C & -0.105507 & -0.061321 & 0.204095 & $0.177^{\star}$ \\
\hline C48 & $0.4942(7)$ & $0.0012(6)$ & $-0.1291(3)$ & $0.0611(17)$ \\
\hline H48A & 0.511144 & -0.076731 & -0.116634 & $0.092^{\star}$ \\
\hline H48B & 0.571967 & 0.046052 & -0.142700 & $0.092^{\star}$ \\
\hline H48C & 0.430502 & -0.023364 & -0.167034 & $0.092^{\star}$ \\
\hline & & & & \\
\hline
\end{tabular}

Atomic displacement parameters $\left(\AA^{2}\right)$ for (8)

\begin{tabular}{|l|l|l|l|l|l|l|}
\hline & $U^{11}$ & $U^{22}$ & $U^{\beta 3}$ & $U^{12}$ & $U^{13}$ & $U^{23}$ \\
\hline
\end{tabular}




\begin{tabular}{|c|c|c|c|c|c|c|}
\hline Os1 & $\begin{array}{l}0.02462 \\
(10)\end{array}$ & $\begin{array}{l}0.02413 \\
(10)\end{array}$ & $\begin{array}{l}0.02483 \\
(10)\end{array}$ & $\begin{array}{l}0.00310 \\
(7)\end{array}$ & $\begin{array}{l}-0.00128 \\
(6)\end{array}$ & $\begin{array}{l}0.00475 \\
(7)\end{array}$ \\
\hline $\mathrm{P} 1$ & $0.0245(5)$ & $0.0219(5)$ & $0.0241(5)$ & $0.0042(4)$ & $\begin{array}{l}-0.0020 \\
(4)\end{array}$ & $0.0041(4)$ \\
\hline O1 & $0.065(3)$ & $\begin{array}{l}0.0304 \\
(18)\end{array}$ & $0.040(2)$ & $\begin{array}{l}0.0011 \\
(17)\end{array}$ & $\begin{array}{l}-0.0208 \\
(18)\end{array}$ & $\begin{array}{l}0.0111 \\
(15)\end{array}$ \\
\hline C1 & $0.066(4)$ & $0.040(3)$ & $0.048(3)$ & $-0.005(3)$ & $-0.016(3)$ & $0.024(3)$ \\
\hline Os2 & $\begin{array}{l}0.02504 \\
(10)\end{array}$ & $\begin{array}{l}0.02498 \\
(10)\end{array}$ & $\begin{array}{l}0.02367 \\
(10)\end{array}$ & $\begin{array}{l}0.00369 \\
(7)\end{array}$ & $\begin{array}{l}-0.00202 \\
(6)\end{array}$ & $\begin{array}{l}0.00448 \\
(7)\end{array}$ \\
\hline $\mathrm{O} 2$ & $0.046(2)$ & $\begin{array}{l}0.0348 \\
(18)\end{array}$ & $\begin{array}{l}0.0338 \\
(18)\end{array}$ & $\begin{array}{l}-0.0027 \\
(15)\end{array}$ & $\begin{array}{l}-0.0078 \\
(15)\end{array}$ & $\begin{array}{l}0.0124 \\
(14)\end{array}$ \\
\hline P2 & $0.0238(5)$ & $0.0291(6)$ & $0.0215(5)$ & $0.0043(4)$ & $\begin{array}{l}-0.0015 \\
(4)\end{array}$ & $0.0034(4)$ \\
\hline C2 & $0.035(3)$ & $0.055(3)$ & $0.033(3)$ & $0.012(2)$ & $0.003(2)$ & $0.002(2)$ \\
\hline C3 & $0.034(3)$ & $0.045(3)$ & $0.041(3)$ & $-0.002(2)$ & $0.003(2)$ & $-0.003(2)$ \\
\hline O3 & $\begin{array}{l}0.0302 \\
(18)\end{array}$ & $0.050(2)$ & $\begin{array}{l}0.0273 \\
(16)\end{array}$ & $\begin{array}{l}0.0086 \\
(15)\end{array}$ & $\begin{array}{l}-0.0027 \\
(13)\end{array}$ & $\begin{array}{l}-0.0003 \\
(15)\end{array}$ \\
\hline C4 & $0.038(3)$ & $0.033(3)$ & $0.046(3)$ & $0.006(2)$ & $-0.008(2)$ & $0.008(2)$ \\
\hline $\mathrm{O} 4$ & $\begin{array}{l}0.0290 \\
(17)\end{array}$ & $0.052(2)$ & $\begin{array}{l}0.0298 \\
(17)\end{array}$ & $\begin{array}{l}0.0134 \\
(15)\end{array}$ & $\begin{array}{l}-0.0041 \\
(13)\end{array}$ & $\begin{array}{l}-0.0032 \\
(15)\end{array}$ \\
\hline O5 & $0.040(2)$ & $0.105(4)$ & $0.050(2)$ & $-0.002(2)$ & $\begin{array}{l}0.0157 \\
(19)\end{array}$ & $-0.017(2)$ \\
\hline C5 & $0.035(3)$ & $0.037(3)$ & $0.037(3)$ & $0.009(2)$ & $-0.010(2)$ & $-0.002(2)$ \\
\hline $\mathrm{O} 6$ & $0.075(3)$ & $0.046(2)$ & $0.086(3)$ & $0.014(2)$ & $-0.026(2)$ & $0.031(2)$ \\
\hline C6 & $0.041(3)$ & $0.039(3)$ & $0.039(3)$ & $-0.007(2)$ & $-0.011(2)$ & $0.011(2)$ \\
\hline $\mathrm{O} 7$ & $0.038(2)$ & $0.060(3)$ & $0.063(3)$ & $\begin{array}{l}0.0250 \\
(19)\end{array}$ & $\begin{array}{l}-0.0022 \\
(18)\end{array}$ & $-0.011(2)$ \\
\hline $\mathrm{C7}$ & $0.023(2)$ & $0.026(2)$ & $0.027(2)$ & 0.0043 & -0.0026 & 0.0022 \\
\hline
\end{tabular}




\begin{tabular}{|c|c|c|c|c|c|c|}
\hline & & & & (16) & (16) & (17) \\
\hline C8 & $0.037(3)$ & $0.030(2)$ & $0.026(2)$ & $\begin{array}{l}0.0077 \\
(19)\end{array}$ & $\begin{array}{l}0.0042 \\
(18)\end{array}$ & $\begin{array}{l}0.0085 \\
(18)\end{array}$ \\
\hline O8 & $0.098(4)$ & $0.056(3)$ & $0.068(3)$ & $-0.028(2)$ & $-0.035(3)$ & $0.041(2)$ \\
\hline C9 & $0.038(3)$ & $0.029(2)$ & $0.032(2)$ & $\begin{array}{l}0.0123 \\
(19)\end{array}$ & $\begin{array}{l}0.0020 \\
(19)\end{array}$ & $\begin{array}{l}0.0114 \\
(18)\end{array}$ \\
\hline C10 & $0.044(3)$ & $0.034(3)$ & 0.039 (3) & $0.015(2)$ & $0.012(2)$ & 0.007 (2) \\
\hline C11 & $0.089(5)$ & $0.050(3)$ & $0.026(2)$ & $0.034(3)$ & $0.016(3)$ & $0.007(2)$ \\
\hline C12 & $0.072(4)$ & $0.042(3)$ & $0.027(2)$ & 0.027 (3) & $0.008(2)$ & $0.009(2)$ \\
\hline C13 & $0.030(2)$ & $0.026(2)$ & $0.021(2)$ & $\begin{array}{l}0.0042 \\
(17)\end{array}$ & $\begin{array}{l}-0.0055 \\
(16)\end{array}$ & $\begin{array}{l}0.0015 \\
(16)\end{array}$ \\
\hline C14 & $0.029(2)$ & $0.030(2)$ & $0.037(2)$ & $\begin{array}{l}0.0031 \\
(18)\end{array}$ & $\begin{array}{l}-0.0066 \\
(19)\end{array}$ & $\begin{array}{l}0.0070 \\
(19)\end{array}$ \\
\hline C15 & 0.031 (3) & $0.041(3)$ & 0.049 (3) & $-0.005(2)$ & $-0.008(2)$ & $0.021(2)$ \\
\hline C16 & $0.030(3)$ & $0.063(4)$ & 0.041 (3) & $0.005(2)$ & $0.001(2)$ & $0.028(3)$ \\
\hline C17 & $0.030(2)$ & $0.053(3)$ & $0.037(3)$ & $0.016(2)$ & $0.003(2)$ & $0.009(2)$ \\
\hline C18 & $0.030(2)$ & $0.033(2)$ & 0.037 (3) & $\begin{array}{l}0.0085 \\
(19)\end{array}$ & $\begin{array}{l}-0.0021 \\
(19)\end{array}$ & $0.004(2)$ \\
\hline C19 & $0.030(2)$ & $0.023(2)$ & $0.025(2)$ & $\begin{array}{l}0.0031 \\
(17)\end{array}$ & $\begin{array}{l}-0.0061 \\
(17)\end{array}$ & $\begin{array}{l}0.0041 \\
(16)\end{array}$ \\
\hline C20 & $0.035(3)$ & $0.038(3)$ & 0.037 (3) & $0.002(2)$ & $-0.003(2)$ & $0.016(2)$ \\
\hline C21 & 0.049 (3) & $0.036(3)$ & $0.040(3)$ & $0.000(2)$ & $0.001(2)$ & $0.013(2)$ \\
\hline C22 & $0.058(3)$ & $0.038(3)$ & 0.037 (3) & $0.012(2)$ & $-0.004(2)$ & $0.014(2)$ \\
\hline C23 & $0.045(3)$ & $0.045(3)$ & $0.042(3)$ & $0.010(2)$ & $-0.016(2)$ & $0.012(2)$ \\
\hline C24 & $0.034(2)$ & $0.034(2)$ & $0.034(2)$ & $\begin{array}{l}0.0031 \\
(19)\end{array}$ & $\begin{array}{l}-0.0024 \\
(19)\end{array}$ & $\begin{array}{l}0.0115 \\
(19)\end{array}$ \\
\hline C25 & $0.030(2)$ & $0.031(2)$ & $0.026(2)$ & 0.0054 & -0.0033 & 0.0055 \\
\hline
\end{tabular}




\begin{tabular}{|c|c|c|c|c|c|c|}
\hline & & & & (18) & (17) & (18) \\
\hline C26 & $0.042(3)$ & $0.034(2)$ & $0.031(2)$ & $0.004(2)$ & $-0.007(2)$ & $\begin{array}{l}0.0045 \\
(19)\end{array}$ \\
\hline C27 & $0.050(3)$ & $0.046(3)$ & $0.036(3)$ & $0.000(2)$ & $-0.006(2)$ & $0.016(2)$ \\
\hline C28 & $0.037(3)$ & $0.040(3)$ & $0.046(3)$ & $-0.003(2)$ & $-0.011(2)$ & $0.018(2)$ \\
\hline C29 & 0.054 (3) & 0.045 (3) & $0.041(3)$ & 0.019 (3) & $-0.007(2)$ & $0.006(2)$ \\
\hline C30 & $0.050(3)$ & $0.048(3)$ & 0.031 (3) & $0.021(2)$ & $-0.006(2)$ & $0.003(2)$ \\
\hline C31 & $0.029(2)$ & $0.037(2)$ & $0.021(2)$ & $\begin{array}{l}0.0067 \\
(19)\end{array}$ & $\begin{array}{l}0.0039 \\
(17)\end{array}$ & $\begin{array}{l}0.0058 \\
(18)\end{array}$ \\
\hline C32 & $0.030(2)$ & $0.042(3)$ & $0.030(2)$ & $0.004(2)$ & $\begin{array}{l}0.0009 \\
(18)\end{array}$ & $0.007(2)$ \\
\hline C33 & $0.041(3)$ & $0.063(4)$ & $0.036(3)$ & $-0.005(2)$ & $0.000(2)$ & $0.023(2)$ \\
\hline C34 & $0.035(3)$ & $0.111(6)$ & $0.053(4)$ & $-0.005(3)$ & $0.001(3)$ & $0.049(4)$ \\
\hline C35 & $0.025(3)$ & $0.130(6)$ & 0.065 (4) & $0.015(3)$ & $0.007(3)$ & $0.060(4)$ \\
\hline C36 & $0.031(3)$ & $0.076(4)$ & 0.041 (3) & $0.014(3)$ & $0.003(2)$ & $0.030(3)$ \\
\hline C37 & $0.027(2)$ & $0.031(2)$ & $0.023(2)$ & $\begin{array}{l}0.0040 \\
(18)\end{array}$ & $\begin{array}{l}-0.0016 \\
(16)\end{array}$ & $\begin{array}{l}0.0051 \\
(17)\end{array}$ \\
\hline C38 & $0.042(3)$ & $0.037(3)$ & $0.029(2)$ & $-0.002(2)$ & $0.009(2)$ & $0.001(2)$ \\
\hline C39 & $0.068(4)$ & $0.031(3)$ & $0.035(3)$ & $0.006(2)$ & $0.006(2)$ & $0.000(2)$ \\
\hline C40 & $0.053(3)$ & $0.043(3)$ & $0.024(2)$ & $0.018(2)$ & $0.010(2)$ & $0.009(2)$ \\
\hline C41 & 0.031 (3) & $0.057(3)$ & $0.032(2)$ & $0.011(2)$ & $\begin{array}{l}0.0092 \\
(19)\end{array}$ & $0.014(2)$ \\
\hline C42 & $0.034(3)$ & $0.040(3)$ & $0.029(2)$ & $0.001(2)$ & $\begin{array}{l}0.0024 \\
(19)\end{array}$ & $0.005(2)$ \\
\hline $\mathrm{C} 43$ & $0.090(5)$ & $0.056(4)$ & $0.057(4)$ & $0.044(4)$ & $0.021(3)$ & $0.012(3)$ \\
\hline C44 & 0.039 (3) & $0.093(5)$ & $0.080(5)$ & $0.001(3)$ & $0.014(3)$ & $0.045(4)$ \\
\hline C45 & $0.084(5)$ & $0.080(5)$ & 0.059 (4) & $0.015(4)$ & $-0.010(3)$ & $0.045(4)$ \\
\hline
\end{tabular}




\begin{tabular}{|l|l|l|l|l|l|l|}
\hline C46 & $0.074(5)$ & $0.048(3)$ & $0.074(4)$ & $0.014(3)$ & $-0.017(4)$ & $0.025(3)$ \\
\hline C47 & $0.036(4)$ & $0.210(11)$ & $0.146(8)$ & $-0.016(5)$ & $0.000(4)$ & $0.138(8)$ \\
\hline C48 & $0.085(5)$ & $0.061(4)$ & $0.044(3)$ & $0.039(4)$ & $0.020(3)$ & $0.007(3)$ \\
\hline
\end{tabular}

Geometric parameters (Å, ) for (8)

\begin{tabular}{|l|l|l|l|}
\hline Os1-P1 & $2.4148(11)$ & $\mathrm{C} 22-\mathrm{C} 45$ & $1.512(7)$ \\
\hline Os1-O1 & $2.138(3)$ & $\mathrm{C} 23-\mathrm{H} 23$ & 0.9500 \\
\hline Os1-Os2 & $2.7387(2)$ & $\mathrm{C} 23-\mathrm{C} 24$ & $1.403(6)$ \\
\hline Os1-C3 & $1.845(5)$ & $\mathrm{C} 24-\mathrm{H} 24$ & 0.9500 \\
\hline Os1-O3 & $2.137(3)$ & $\mathrm{C} 25-\mathrm{C} 26$ & $1.395(6)$ \\
\hline Os1-C4 & $1.844(5)$ & $\mathrm{C} 25-\mathrm{C} 30$ & $1.392(6)$ \\
\hline $\mathrm{P} 1-\mathrm{C} 7$ & $1.825(4)$ & $\mathrm{C} 26-\mathrm{H} 26$ & 0.9500 \\
\hline $\mathrm{P} 1-\mathrm{C} 13$ & $1.818(5)$ & $\mathrm{C} 26-\mathrm{C} 27$ & $1.378(7)$ \\
\hline $\mathrm{P} 1-\mathrm{C} 19$ & $1.831(4)$ & $\mathrm{C} 27-\mathrm{H} 27$ & 0.9500 \\
\hline O1-C1 & $1.248(6)$ & $\mathrm{C} 27-\mathrm{C} 28$ & $1.365(8)$ \\
\hline C1-H1 & 0.9500 & $\mathrm{C} 28-\mathrm{C} 29$ & $1.397(7)$ \\
\hline C1-O2 & $1.242(6)$ & $\mathrm{C} 28-\mathrm{C} 46$ & $1.528(7)$ \\
\hline Os2-O2 & $2.134(3)$ & $\mathrm{C} 29-\mathrm{H} 29$ & 0.9500 \\
\hline Os2-P2 & $2.4325(11)$ & $\mathrm{C} 29-\mathrm{C} 30$ & $1.374(7)$ \\
\hline Os2-O4 & $2.160(3)$ & $\mathrm{C} 30-\mathrm{H} 30$ & 0.9500 \\
\hline Os2-C5 & $1.844(5)$ & $\mathrm{C} 31-\mathrm{C} 32$ & $1.390(6)$ \\
\hline Os2-C6 & $1.834(5)$ & $\mathrm{C} 31-\mathrm{C} 36$ & $1.390(6)$ \\
\hline P2-C25 & $1.824(4)$ & $\mathrm{C} 32-\mathrm{H} 32$ & 0.9500 \\
\hline P2-C31 & $1.815(5)$ & $\mathrm{C} 32-\mathrm{C} 33$ & $1.385(7)$ \\
\hline P2-C37 & $1.826(4)$ & $\mathrm{C} 33-\mathrm{H} 33$ & 0.9500 \\
\hline C2-H2 & 0.9500 & $\mathrm{C} 33-\mathrm{C} 34$ & $1.386(8)$ \\
\hline & & & \\
\hline
\end{tabular}




\begin{tabular}{|l|l|l|l|}
\hline C2-O3 & $1.253(6)$ & C34-C35 & $1.359(9)$ \\
\hline C2-O4 & $1.243(6)$ & C34-C47 & $1.520(8)$ \\
\hline C3-O5 & $1.148(6)$ & C35-H35 & 0.9500 \\
\hline C4-O6 & $1.145(6)$ & C35-C36 & $1.400(8)$ \\
\hline C5-O7 & $1.155(6)$ & C36-H36 & 0.9500 \\
\hline C6-O8 & $1.161(6)$ & C37-C38 & $1.388(6)$ \\
\hline C7-C8 & $1.390(6)$ & C37-C42 & $1.387(6)$ \\
\hline C7-C12 & $1.390(6)$ & C38-H38 & 0.9500 \\
\hline C8-H8 & 0.9500 & C38-C39 & $1.395(7)$ \\
\hline C8-C9 & $1.384(6)$ & C39-H39 & 0.9500 \\
\hline C9-H9 & 0.9500 & C39-C40 & $1.379(7)$ \\
\hline C9-C10 & $1.378(6)$ & C40-C41 & $1.377(7)$ \\
\hline C10-C11 & $1.381(7)$ & C40-C48 & $1.518(7)$ \\
\hline C10-C43 & $1.499(7)$ & C41-H41 & 0.9500 \\
\hline C11-H11 & 0.9500 & C41-C42 & $1.374(6)$ \\
\hline C11-C12 & $1.378(7)$ & C42-H42 & 0.9500 \\
\hline C12-H12 & 0.9500 & C43-H43A & 0.9800 \\
\hline C13-C14 & $1.400(6)$ & C43-H43B & 0.9800 \\
\hline C13-C18 & $1.397(6)$ & C43-H43C & 0.9800 \\
\hline C14-H14 & 0.9500 & C44-H44A & 0.9800 \\
\hline C14-C15 & $1.393(7)$ & C44-H44B & 0.9800 \\
\hline C15-H15 & 0.9500 & C44-H44C & 0.9800 \\
\hline C15-C16 & $1.374(8)$ & C45-H45A & 0.9800 \\
\hline C16-C17 & $1.387(8)$ & C45-H45B & 0.9800 \\
\hline C16-C44 & $1.514(7)$ & C45-H45C & 0.9800 \\
\hline
\end{tabular}




\begin{tabular}{|c|c|c|c|}
\hline $\mathrm{C} 17-\mathrm{H} 17$ & 0.9500 & $\mathrm{C} 46-\mathrm{H} 46 \mathrm{~A}$ & 0.9800 \\
\hline C17-C18 & $1.373(7)$ & $\mathrm{C} 46-\mathrm{H} 46 \mathrm{~B}$ & 0.9800 \\
\hline $\mathrm{C} 18-\mathrm{H} 18$ & 0.9500 & $\mathrm{C} 46-\mathrm{H} 46 \mathrm{C}$ & 0.9800 \\
\hline C19-C20 & $1.394(6)$ & $\mathrm{C} 47-\mathrm{H} 47 \mathrm{~A}$ & 0.9800 \\
\hline C19-C24 & $1.381(6)$ & $\mathrm{C} 47-\mathrm{H} 47 \mathrm{~B}$ & 0.9800 \\
\hline $\mathrm{C} 20-\mathrm{H} 20$ & 0.9500 & $\mathrm{C} 47-\mathrm{H} 47 \mathrm{C}$ & 0.9800 \\
\hline $\mathrm{C} 20-\mathrm{C} 21$ & $1.378(7)$ & $\mathrm{C} 48-\mathrm{H} 48 \mathrm{~A}$ & 0.9800 \\
\hline $\mathrm{C} 21-\mathrm{H} 21$ & 0.9500 & $\mathrm{C} 48-\mathrm{H} 48 \mathrm{~B}$ & 0.9800 \\
\hline $\mathrm{C} 21-\mathrm{C} 22$ & $1.396(7)$ & $\mathrm{C} 48-\mathrm{H} 48 \mathrm{C}$ & 0.9800 \\
\hline $\mathrm{C} 22-\mathrm{C} 23$ & $1.380(8)$ & & \\
\hline $\mathrm{P} 1-\mathrm{Os} 1-\mathrm{Os} 2$ & $164.48(3)$ & $\mathrm{C} 22-\mathrm{C} 21-\mathrm{H} 21$ & 119.7 \\
\hline $\mathrm{O} 1-\mathrm{O} 1-\mathrm{P} 1$ & $89.31(9)$ & $\mathrm{C} 21-\mathrm{C} 22-\mathrm{C} 45$ & $120.6(5)$ \\
\hline O1-Os1-Os2 & $81.48(9)$ & $\mathrm{C} 23-\mathrm{C} 22-\mathrm{C} 21$ & $118.4(5)$ \\
\hline $\mathrm{C} 3-\mathrm{O} 1-\mathrm{P} 1$ & 99.28 (16) & $\mathrm{C} 23-\mathrm{C} 22-\mathrm{C} 45$ & $121.0(5)$ \\
\hline $\mathrm{C} 3-\mathrm{Os} 1-\mathrm{O} 1$ & $89.2(2)$ & $\mathrm{C} 22-\mathrm{C} 23-\mathrm{H} 23$ & 119.4 \\
\hline $\mathrm{C} 3-\mathrm{Os} 1-\mathrm{Os} 2$ & $93.09(16)$ & $\mathrm{C} 22-\mathrm{C} 23-\mathrm{C} 24$ & $121.2(5)$ \\
\hline $\mathrm{C} 3-\mathrm{Os} 1-\mathrm{O} 3$ & $173.12(19)$ & $\mathrm{C} 24-\mathrm{C} 23-\mathrm{H} 23$ & 119.4 \\
\hline $\mathrm{O} 3-\mathrm{O} 1-\mathrm{P} 1$ & $84.04(9)$ & $\mathrm{C} 19-\mathrm{C} 24-\mathrm{C} 23$ & $120.1(5)$ \\
\hline $\mathrm{O} 3-\mathrm{O} 1-\mathrm{O} 1$ & $84.79(15)$ & $\mathrm{C} 19-\mathrm{C} 24-\mathrm{H} 24$ & 120.0 \\
\hline O3-Os1-Os2 & $82.71(8)$ & $\mathrm{C} 23-\mathrm{C} 24-\mathrm{H} 24$ & 120.0 \\
\hline $\mathrm{C} 4-\mathrm{O} 1-\mathrm{P} 1$ & $93.97(15)$ & C26-C25-P2 & $123.9(4)$ \\
\hline $\mathrm{C} 4-\mathrm{Os} 1-\mathrm{O} 1$ & $176.54(17)$ & $\mathrm{C} 30-\mathrm{C} 25-\mathrm{P} 2$ & $118.3(3)$ \\
\hline $\mathrm{C} 4-\mathrm{Os} 1-\mathrm{Os} 2$ & $95.56(15)$ & $\mathrm{C} 30-\mathrm{C} 25-\mathrm{C} 26$ & $117.8(4)$ \\
\hline $\mathrm{C} 4-\mathrm{Os} 1-\mathrm{C} 3$ & $89.2(2)$ & $\mathrm{C} 25-\mathrm{C} 26-\mathrm{H} 26$ & 119.8 \\
\hline
\end{tabular}




\begin{tabular}{|c|c|c|c|}
\hline $\mathrm{C} 4-\mathrm{O} 1-\mathrm{O} 3$ & $96.7(2)$ & $\mathrm{C} 27-\mathrm{C} 26-\mathrm{C} 25$ & $120.5(5)$ \\
\hline $\mathrm{C} 7-\mathrm{P} 1-\mathrm{Os} 1$ & $115.13(13)$ & $\mathrm{C} 27-\mathrm{C} 26-\mathrm{H} 26$ & 119.8 \\
\hline C7-P1-C19 & $103.86(19)$ & $\mathrm{C} 26-\mathrm{C} 27-\mathrm{H} 27$ & 119.1 \\
\hline $\mathrm{C} 13-\mathrm{P} 1-\mathrm{Os} 1$ & $115.62(13)$ & $\mathrm{C} 28-\mathrm{C} 27-\mathrm{C} 26$ & $121.7(5)$ \\
\hline $\mathrm{C} 13-\mathrm{P} 1-\mathrm{C} 7$ & 103.79 (19) & $\mathrm{C} 28-\mathrm{C} 27-\mathrm{H} 27$ & 119.1 \\
\hline C13-P1-C19 & 103.59 (19) & C27-C28-C29 & $118.3(5)$ \\
\hline $\mathrm{C} 19-\mathrm{P} 1-\mathrm{Os} 1$ & $113.40(14)$ & $\mathrm{C} 27-\mathrm{C} 28-\mathrm{C} 46$ & $121.6(5)$ \\
\hline $\mathrm{C} 1-\mathrm{O} 1-\mathrm{Os} 1$ & $121.4(3)$ & C29-C28-C46 & $120.1(5)$ \\
\hline $\mathrm{O} 1-\mathrm{C} 1-\mathrm{H} 1$ & 115.6 & C28-C29-H29 & 119.7 \\
\hline $\mathrm{O} 2-\mathrm{C} 1-\mathrm{O} 1$ & $128.8(5)$ & $\mathrm{C} 30-\mathrm{C} 29-\mathrm{C} 28$ & $120.6(5)$ \\
\hline $\mathrm{O} 2-\mathrm{C} 1-\mathrm{H} 1$ & 115.6 & $\mathrm{C} 30-\mathrm{C} 29-\mathrm{H} 29$ & 119.7 \\
\hline $\mathrm{O} 2-\mathrm{Os} 2-\mathrm{Os} 1$ & $83.19(9)$ & $\mathrm{C} 25-\mathrm{C} 30-\mathrm{H} 30$ & 119.5 \\
\hline $\mathrm{O} 2-\mathrm{Os} 2-\mathrm{P} 2$ & $84.97(9)$ & C29-C30-C25 & $121.1(5)$ \\
\hline $\mathrm{O} 2-\mathrm{O} 2-\mathrm{O} 4$ & $84.76(14)$ & $\mathrm{C} 29-\mathrm{C} 30-\mathrm{H} 30$ & 119.5 \\
\hline $\mathrm{P} 2-\mathrm{Os} 2-\mathrm{Os} 1$ & $165.90(3)$ & $\mathrm{C} 32-\mathrm{C} 31-\mathrm{P} 2$ & $119.0(3)$ \\
\hline O4-Os2-Os 1 & $82.05(8)$ & C32-C31-C36 & $117.7(4)$ \\
\hline O4-Os2-P2 & $89.27(9)$ & C36-C31-P2 & $123.3(4)$ \\
\hline C5-Os2-Os1 & $92.43(14)$ & C31-C32-H32 & 119.2 \\
\hline $\mathrm{C} 5-\mathrm{Os} 2-\mathrm{O} 2$ & $95.17(19)$ & $\mathrm{C} 33-\mathrm{C} 32-\mathrm{C} 31$ & $121.6(5)$ \\
\hline $\mathrm{C} 5-\mathrm{Os} 2-\mathrm{P} 2$ & $96.25(14)$ & $\mathrm{C} 33-\mathrm{C} 32-\mathrm{H} 32$ & 119.2 \\
\hline $\mathrm{C} 5-\mathrm{Os} 2-\mathrm{O} 4$ & $174.46(16)$ & $\mathrm{C} 32-\mathrm{C} 33-\mathrm{H} 33$ & 120.0 \\
\hline $\mathrm{C} 6-\mathrm{Os} 2-\mathrm{Os} 1$ & $91.97(15)$ & $\mathrm{C} 32-\mathrm{C} 33-\mathrm{C} 34$ & $120.0(5)$ \\
\hline $\mathrm{C} 6-\mathrm{Os} 2-\mathrm{O} 2$ & 173.65 (18) & C34-C33-H33 & 120.0 \\
\hline $\mathrm{C} 6-\mathrm{Os} 2-\mathrm{P} 2$ & $99.24(15)$ & C33-C34-C47 & $120.4(6)$ \\
\hline $\mathrm{C} 6-\mathrm{Os} 2-\mathrm{O} 4$ & $90.5(2)$ & C35-C34-C33 & $119.0(5)$ \\
\hline
\end{tabular}




\begin{tabular}{|l|l|l|l|}
\hline C6-Os2-C5 & $89.1(2)$ & C35-C34-C47 & $120.5(6)$ \\
\hline C1-O2-Os2 & $119.8(3)$ & C34-C35-H35 & 119.3 \\
\hline C25-P2-Os2 & $116.17(14)$ & C34-C35-C36 & $121.4(5)$ \\
\hline C25-P2-C37 & $103.6(2)$ & C36-C35-H35 & 119.3 \\
\hline C31-P2-Os2 & $115.98(14)$ & C31-C36-C35 & $120.1(5)$ \\
\hline C31-P2-C25 & $103.8(2)$ & C31-C36-H36 & 119.9 \\
\hline C31-P2-C37 & $104.1(2)$ & C35-C36-H36 & 119.9 \\
\hline C37-P2-Os2 & $111.77(14)$ & C38-C37-P2 & $123.8(3)$ \\
\hline O3-C2-H2 & 115.8 & C42-C37-P2 & $117.3(3)$ \\
\hline O4-C2-H2 & 115.8 & C42-C37-C38 & $118.8(4)$ \\
\hline O4-C2-O3 & $128.4(5)$ & C37-C38-H38 & 120.0 \\
\hline O5-C3-Os1 & $177.7(6)$ & C37-C38-C39 & $120.0(5)$ \\
\hline C2-O3-Os1 & $120.6(3)$ & C39-C38-H38 & 120.0 \\
\hline O6-C4-Os1 & $178.0(5)$ & C38-C39-H39 & 119.5 \\
\hline C2-O4-Os2 & $120.7(3)$ & C40-C39-C38 & $121.0(5)$ \\
\hline O7-C5-Os2 & $177.9(4)$ & C40-C39-H39 & 119.5 \\
\hline O8-C6-Os2 & $177.8(5)$ & C39-C40-C48 & $120.9(5)$ \\
\hline C8-C7-P1 & $118.3(3)$ & C41-C40-C39 & $118.1(4)$ \\
\hline C12-C7-P1 & $123.7(3)$ & C41-C40-C48 & $121.0(5)$ \\
\hline C12-C7-C8 & $118.0(4)$ & C40-C41-H41 & 119.1 \\
\hline C7-C8-H8 & 119.5 & C42-C41-C40 & $121.9(5)$ \\
\hline C9-C8-C7 & $120.9(4)$ & C42-C41-H41 & 119.1 \\
\hline C9-C8-H8 & 119.5 & C37-C42-H42 & 119.9 \\
\hline C8-C9-H9 & 119.5 & C41-C42-C37 & $120.2(4)$ \\
\hline C10-C9-C8 & $120.9(4)$ & C41-C42-H42 & 119.9 \\
\hline
\end{tabular}




\begin{tabular}{|c|c|c|c|}
\hline $\mathrm{C} 10-\mathrm{C} 9-\mathrm{H} 9$ & 119.5 & $\mathrm{C} 10-\mathrm{C} 43-\mathrm{H} 43 \mathrm{~A}$ & 109.5 \\
\hline $\mathrm{C} 9-\mathrm{C} 10-\mathrm{C} 11$ & $118.0(4)$ & $\mathrm{C} 10-\mathrm{C} 43-\mathrm{H} 43 \mathrm{~B}$ & 109.5 \\
\hline $\mathrm{C} 9-\mathrm{C} 10-\mathrm{C} 43$ & $120.6(5)$ & $\mathrm{C} 10-\mathrm{C} 43-\mathrm{H} 43 \mathrm{C}$ & 109.5 \\
\hline $\mathrm{C} 11-\mathrm{C} 10-\mathrm{C} 43$ & $121.4(5)$ & $\begin{array}{l}\mathrm{H} 43 \mathrm{~A}-\mathrm{C} 43- \\
\mathrm{H} 43 \mathrm{~B}\end{array}$ & 109.5 \\
\hline $\mathrm{C} 10-\mathrm{C} 11-\mathrm{H} 11$ & 119.1 & $\begin{array}{l}\mathrm{H} 43 \mathrm{~A}-\mathrm{C} 43- \\
\mathrm{H} 43 \mathrm{C}\end{array}$ & 109.5 \\
\hline $\mathrm{C} 12-\mathrm{C} 11-\mathrm{C} 10$ & $121.9(5)$ & $\begin{array}{l}\mathrm{H} 43 \mathrm{~B}-\mathrm{C} 43- \\
\mathrm{H} 43 \mathrm{C}\end{array}$ & 109.5 \\
\hline $\mathrm{C} 12-\mathrm{C} 11-\mathrm{H} 11$ & 119.1 & $\mathrm{C} 16-\mathrm{C} 44-\mathrm{H} 44 \mathrm{~A}$ & 109.5 \\
\hline $\mathrm{C} 7-\mathrm{C} 12-\mathrm{H} 12$ & 119.9 & $\mathrm{C} 16-\mathrm{C} 44-\mathrm{H} 44 \mathrm{~B}$ & 109.5 \\
\hline $\mathrm{C} 11-\mathrm{C} 12-\mathrm{C} 7$ & $120.3(5)$ & $\mathrm{C} 16-\mathrm{C} 44-\mathrm{H} 44 \mathrm{C}$ & 109.5 \\
\hline $\mathrm{C} 11-\mathrm{C} 12-\mathrm{H} 12$ & 119.9 & $\begin{array}{l}\mathrm{H} 44 \mathrm{~A}-\mathrm{C} 44- \\
\mathrm{H} 44 \mathrm{~B}\end{array}$ & 109.5 \\
\hline $\mathrm{C} 14-\mathrm{C} 13-\mathrm{P} 1$ & $123.6(3)$ & $\begin{array}{l}\mathrm{H} 44 \mathrm{~A}-\mathrm{C} 44- \\
\mathrm{H} 44 \mathrm{C}\end{array}$ & 109.5 \\
\hline $\mathrm{C} 18-\mathrm{C} 13-\mathrm{P} 1$ & $118.3(3)$ & $\begin{array}{l}\mathrm{H} 44 \mathrm{~B}-\mathrm{C} 44- \\
\mathrm{H} 44 \mathrm{C}\end{array}$ & 109.5 \\
\hline $\mathrm{C} 18-\mathrm{C} 13-\mathrm{C} 14$ & $118.1(4)$ & $\mathrm{C} 22-\mathrm{C} 45-\mathrm{H} 45 \mathrm{~A}$ & 109.5 \\
\hline $\mathrm{C} 13-\mathrm{C} 14-\mathrm{H} 14$ & 120.1 & C22-C45-H45B & 109.5 \\
\hline $\mathrm{C} 15-\mathrm{C} 14-\mathrm{C} 13$ & $119.9(4)$ & $\mathrm{C} 22-\mathrm{C} 45-\mathrm{H} 45 \mathrm{C}$ & 109.5 \\
\hline $\mathrm{C} 15-\mathrm{C} 14-\mathrm{H} 14$ & 120.1 & $\begin{array}{l}\mathrm{H} 45 \mathrm{~A}-\mathrm{C} 45- \\
\mathrm{H} 45 \mathrm{~B}\end{array}$ & 109.5 \\
\hline $\mathrm{C} 14-\mathrm{C} 15-\mathrm{H} 15$ & 119.1 & $\begin{array}{l}\mathrm{H} 45 \mathrm{~A}-\mathrm{C} 45- \\
\mathrm{H} 45 \mathrm{C}\end{array}$ & 109.5 \\
\hline $\mathrm{C} 16-\mathrm{C} 15-\mathrm{C} 14$ & $121.9(5)$ & $\begin{array}{l}\mathrm{H} 45 \mathrm{~B}-\mathrm{C} 45- \\
\mathrm{H} 45 \mathrm{C}\end{array}$ & 109.5 \\
\hline $\mathrm{C} 16-\mathrm{C} 15-\mathrm{H} 15$ & 119.1 & $\mathrm{C} 28-\mathrm{C} 46-\mathrm{H} 46 \mathrm{~A}$ & 109.5 \\
\hline
\end{tabular}




\begin{tabular}{|c|c|c|c|}
\hline $\mathrm{C} 15-\mathrm{C} 16-\mathrm{C} 17$ & $117.7(5)$ & $\mathrm{C} 28-\mathrm{C} 46-\mathrm{H} 46 \mathrm{~B}$ & 109.5 \\
\hline $\mathrm{C} 15-\mathrm{C} 16-\mathrm{C} 44$ & $122.2(5)$ & $\mathrm{C} 28-\mathrm{C} 46-\mathrm{H} 46 \mathrm{C}$ & 109.5 \\
\hline C17-C16-C44 & $120.1(5)$ & $\begin{array}{l}\mathrm{H} 46 \mathrm{~A}-\mathrm{C} 46- \\
\mathrm{H} 46 \mathrm{~B}\end{array}$ & 109.5 \\
\hline $\mathrm{C} 16-\mathrm{C} 17-\mathrm{H} 17$ & 119.1 & $\begin{array}{l}\mathrm{H} 46 \mathrm{~A}-\mathrm{C} 46- \\
\mathrm{H} 46 \mathrm{C}\end{array}$ & 109.5 \\
\hline $\mathrm{C} 18-\mathrm{C} 17-\mathrm{C} 16$ & $121.8(5)$ & $\begin{array}{l}\mathrm{H} 46 \mathrm{~B}-\mathrm{C} 46- \\
\mathrm{H} 46 \mathrm{C}\end{array}$ & 109.5 \\
\hline $\mathrm{C} 18-\mathrm{C} 17-\mathrm{H} 17$ & 119.1 & C34-C47-H47A & 109.5 \\
\hline $\mathrm{C} 13-\mathrm{C} 18-\mathrm{H} 18$ & 119.7 & C34-C47-H47B & 109.5 \\
\hline $\mathrm{C} 17-\mathrm{C} 18-\mathrm{C} 13$ & $120.6(5)$ & C34-C47-H47C & 109.5 \\
\hline $\mathrm{C} 17-\mathrm{C} 18-\mathrm{H} 18$ & 119.7 & $\begin{array}{l}\mathrm{H} 47 \mathrm{~A}-\mathrm{C} 47- \\
\mathrm{H} 47 \mathrm{~B}\end{array}$ & 109.5 \\
\hline $\mathrm{C} 20-\mathrm{C} 19-\mathrm{P} 1$ & $118.2(3)$ & $\begin{array}{l}\mathrm{H} 47 \mathrm{~A}-\mathrm{C} 47- \\
\mathrm{H} 47 \mathrm{C}\end{array}$ & 109.5 \\
\hline $\mathrm{C} 24-\mathrm{C} 19-\mathrm{P} 1$ & $123.2(3)$ & $\begin{array}{l}\mathrm{H} 47 \mathrm{~B}-\mathrm{C} 47- \\
\mathrm{H} 47 \mathrm{C}\end{array}$ & 109.5 \\
\hline C24-C19-C20 & $118.6(4)$ & $\mathrm{C} 40-\mathrm{C} 48-\mathrm{H} 48 \mathrm{~A}$ & 109.5 \\
\hline $\mathrm{C} 19-\mathrm{C} 20-\mathrm{H} 20$ & 119.4 & $\mathrm{C} 40-\mathrm{C} 48-\mathrm{H} 48 \mathrm{~B}$ & 109.5 \\
\hline C21-C20-C19 & $121.2(5)$ & $\mathrm{C} 40-\mathrm{C} 48-\mathrm{H} 48 \mathrm{C}$ & 109.5 \\
\hline $\mathrm{C} 21-\mathrm{C} 20-\mathrm{H} 20$ & 119.4 & $\begin{array}{l}\mathrm{H} 48 \mathrm{~A}-\mathrm{C} 48- \\
\mathrm{H} 48 \mathrm{~B}\end{array}$ & 109.5 \\
\hline $\mathrm{C} 20-\mathrm{C} 21-\mathrm{H} 21$ & 119.7 & $\begin{array}{l}\mathrm{H} 48 \mathrm{~A}-\mathrm{C} 48- \\
\mathrm{H} 48 \mathrm{C}\end{array}$ & 109.5 \\
\hline $\mathrm{C} 20-\mathrm{C} 21-\mathrm{C} 22$ & $120.5(5)$ & $\begin{array}{l}\mathrm{H} 48 \mathrm{~B}-\mathrm{C} 48- \\
\mathrm{H} 48 \mathrm{C}\end{array}$ & 109.5 \\
\hline
\end{tabular}

Document origin: pub/CIF [Westrip, S. P. (2010). J. Apply. Cryst., 43, 920-925]. 
